\title{
Bicyclic picomolar OGA inhibitors enable chemoproteomic mapping of its endogenous post-translational modifications.
}

Manuel González-Cuesta, ${ }^{a}$ Peter Sidhu, ${ }^{\mathrm{b}, \mathrm{c}}$ Roger A. Ashmus, ${ }^{\mathrm{b}}$ Alexandra Males, ${ }^{\mathrm{d}}$ Cameron Proceviat, ${ }^{\mathrm{b}}$ Zarina Madden, ${ }^{b}$ Jason C. Rogalski, ${ }^{c}$ Jil A. Busmann, ${ }^{e}$ Leonard J. Foster, ${ }^{c}$ José M. García Fernández, ${ }^{f}$ Gideon

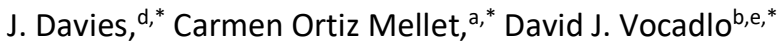

\footnotetext{
${ }^{a}$ Departamento de Química Orgánica, Facultad de Química, Universidad de Sevilla, 41012, Sevilla, Spain. ${ }^{b}$ Department of Chemistry, Simon Fraser University, Burnaby V5A 1S6, British Columbia, Canada.

c Department of Biochemistry and Molecular Biology, University of British Columbia, Vancouver, British Columbia, V6T 1Z4, Canada.

${ }^{d}$ Department of Chemistry. University of York, York YO10 5DD, United Kingdom.

e Department of Molecular Biology and Biochemistry, Simon Fraser University, V5A 1S6, Burnaby, British Columbia, Canada.

f Instituto de Investigaciones Químicas (IIQ), CSIC - Universidad de Sevilla, 41092 Sevilla, Spain.
} 


\section{List of Contents}

General Methods

S3-S8

Materials

S8

Synthetic Procedures and characterization data

S9-S31

Morrison plots for $\boldsymbol{K}_{\mathrm{i}}$ determinations

S32-S35

X-Ray structure solution methods

S36-S38

Chemoproteomic and mapping methods and data

S39-S52

Brain permeability data

S53

Copies of ${ }^{1} \mathrm{H}$ and ${ }^{13} \mathrm{C}$ NMR and MS spectra

S54-S96

References

S97-S99 


\section{General methods}

All reagents and solvents were purchased from commercial sources and used without further purification unless otherwise stated. Thin-layer chromatography (TLC) was carried out on aluminium sheets coated with Silica gel $60 F_{254}$ Merck with visualization by UV light $(\lambda 254 \mathrm{~nm})$ and by charring with $10 \%$ ethanolic $\mathrm{H}_{2} \mathrm{SO}_{4}, 0.1 \%$ ethanolic ninhydrin and heating at $100{ }^{\circ} \mathrm{C}$. With preparative purposes, column chromatography was carried out on Silice 60 A.C.C. Chromagel (SDS 70-200 and 35-70 $\mu$ m). CombiFlash was performed on CombiFlash Rf 200 from Teledyne ISCO. In reference to 'dry load', the residue was dissolved in appropriate solvent then silica gel was added; this solvent was then evaporated to produce the residue adhered to silica which was loaded onto the column in dry form. HPLC was performed on Agilent 1100 series equipped with a variable wavelength UV-Vis detector using either an Eclipse XDB-C1 8 column (3.5 $\mu$ m particle size, $3.0 \times 150 \mathrm{~mm}$ for analytical runs and $5.0 \mu \mathrm{m}, 9.4 \times 250 \mathrm{~mm}$ for semi-preparative scale purifications) or ZORBAX 300SB C8 column (5.0 $\mu \mathrm{m}$ particle size, $9.4^{\prime} 250 \mathrm{~mm}$ for analytical runs and semi-preparative scale purifications) using HPLC grade solvents. Optical rotations were measured at $20 \pm 2{ }^{\circ} \mathrm{C}$ in $1 \mathrm{~cm}$ tubes on a Jasco P-2000 polarimeter using a sodium lamp ( $\lambda 589$ $\mathrm{nm})$. UV spectra were recorded on JASCO V-630 instrument; unit for $\varepsilon$ values: $\mathrm{mm}^{-1} \mathrm{~cm}^{-1}$. Elemental analyses were carried out at the Instituto de Investigaciones Químicas (Sevilla, Spain) using an elemental analyser Leco CHNS-932 o Leco TruSpec CHN. NMR experiments were performed at 300 (75.5), 500 (125.7) and 600 (150) MHz with Bruker 300 ADVANCE, 500 DRX and Bruker Avance 600 equipped with a QNP or TCI cryoprobe (600 MHz). 1D TOCSY, 2D COSY, HMQC and HSQC experiments were used to assist on NMR assignments. The chemical shift values are given in ppm (part per million), using the solvent as internal standard, tetramethylsilane (for $\mathrm{CDCl}_{3}$ ). The values of the coupling constant $(J)$ are measured in $\mathrm{Hz}$. Abbreviations to indicate the multiplicities of the signals are: s (singlet), bs (broad singlet), $d$ (doublet), $\mathrm{t}$ (triplet), q (quartet) and $\mathrm{m}$ (multiplet). Mass spectra were carried out on Micromass AutoSpecQ (for chemical ionization, CI) or Bruker Daltonics Esquire6000 ${ }^{\mathrm{TM}}$ (for electrospray ionization, ESI). For CI mass spectrometry, samples were introduced via solid probe heated from 30 to $280{ }^{\circ} \mathrm{C}$. In the case of ESI, methanol was used as the solvent. High resolution mass spectrometer (HRMS) spectra were recorded on a Bruker MaXis Impact or Agilent Technologies 6210 Time-of-Flight LC/MS spectrometers using positive or negative ESI.

Inhibition assays against commercial $\boldsymbol{\beta}-\mathbf{N}$-acetylglucosaminidases. Inhibition constant $\left(K_{\mathrm{i}}\right)$ values were determined by spectrophotometrically measuring the residual hydrolytic activities of the glycosidases against $p$-nitrophenyl $N$-acetyl- $\beta$-D-glucosaminide. The $K_{\mathrm{m}}$ values for the glycosidases used in the tests and the corresponding working pHs are listed herein: $\beta-\mathrm{N}$ - 
acetylglucosaminidase (from human placenta), $K_{\mathrm{m}}=0.34 \mathrm{mM} \quad(\mathrm{pH} \quad 5.5) ; \quad \beta-\mathrm{N}-$ acetylglucosaminidase (from bovine kidney), $K_{\mathrm{m}}=0.48 \mathrm{mM}$ (pH 5.5). Each assay was performed in phosphate-citrate buffer and the reactions were initiated by addition of the enzyme to a solution of the substrate in the absence or presence of various inhibitor concentrations. The mixture was incubated for 10-30 min at $37{ }^{\circ} \mathrm{C}$ and the reaction was quenched by addition of $1 \mathrm{M} \mathrm{Na}_{2} \mathrm{CO}_{3}$. Reaction times were appropriate to obtain 10-20\% conversion of the substrate in order to achieve linear rates. The absorbance of the resulting mixture was determined at $405 \mathrm{~nm}$. Approximate values of $K_{\mathrm{i}}$ were determined using a fixed concentration of substrate (around the $K_{\mathrm{m}}$ value for the different glycosidases) and various inhibitor concentrations. Full $K_{\mathrm{i}}$ determinations and enzyme inhibition mode were determined from the slope of Lineweaver-Burk plots and double reciprocal analysis using a Microsoft Office Excel 2007 program.

Inhibition assays against hOGA. Inhibition constant $\left(K_{\mathrm{i}}\right)$ values for compounds against hOGA enzyme are determined by measuring the change in fluorescent signal corresponding to the rate of hydrolytic activity against the artificial substrate, Resorufin-N-acetyl- $\beta$-D-glucosaminide. hOGA activity assays were performed in a buffer of $20 \mathrm{mM}$ HEPES, $5 \mathrm{mM}$ EDTA, $150 \mathrm{mM} \mathrm{KCl}$, $\mathrm{pH} 7.1$ and $0.2 \mathrm{nM}[\mathrm{hOGA}] . K_{\mathrm{m}}=25 \mathrm{uM}$ was measured with these conditions. Inhibition assays were run at $0.2 \mathrm{nM}[\mathrm{hOGA}]$ in the same buffer, in the presence or absence of various concentrations of inhibitors and at a fixed substrate concentration of $25 \mu \mathrm{M}$ and $1 \%$ DMSO. First, inhibitor was serially diluted to the desired range of concentrations in 2\% DMSO buffer. Next, $50 \mu \mathrm{L}$ of inhibitor solutions at various concentrations in $2 \%$ DMSO buffer was added to $50 \mu \mathrm{L}$ of $0.8 \mathrm{nM}$ enzyme and allowed to incubate at $25^{\circ} \mathrm{C}$ for 5 minutes. $100 \mu \mathrm{L}$ of $50 \mu \mathrm{M}$ and $1 \%$ DMSO substrate was then added and reaction mixture was immediately mixed and aliquoted in $45 \mu \mathrm{L}$ triplicates to a CORNING 384 well black plate. Fluorescence signal was measured continuously for 20 minutes at $37^{\circ} \mathrm{C}$ in a BioTek Neo 2 Plate reader set at excitation and emission wavelengths of 572 and $610 \mathrm{~nm}$, respectively. Maximal reaction rates for all inhibitor concentrations were calculated within Gen5 BioTek reader software. \% A ctivity was subsequently calculated for each inhibitor concentration against the maximal reaction rate of uninhibited hOGA. GraphPad Prism 2016 was used to approximate the Morrison $K_{\mathrm{i}}$ values for each inhibitor.

Inhibition assays against rhHexA. Inhibition constant $\left(K_{\mathrm{i}}\right)$ values for compounds against recombinant human Hexosaminidase A (HexA) enzyme, purchased from R\&D Systems (cat\# 6237-GH-020), were determined by measuring the fluorescent signal corresponding to the rate of hydrolytic activity against the commercially available artificial substrate, 4-methylumbelliferone$\mathrm{N}$-acetyl- $\beta$-D-glucosaminide. HexA activity assays were performed in a buffer of $100 \mathrm{mM}$ sodium citrate, $250 \mathrm{NaCl}, \mathrm{pH} 4.5$ and $1.0 \mathrm{nM}$ [HexA], then stopped after 20 minutes with a 
solution of 1.0 M Tris, pH 9.5 to enhance fluorescent signal. Preliminary experiments have shown that reaction rates are linear for $20 \mathrm{~min}$ after substrate addition, and that $1.0 \mathrm{M}$ Tris at $\mathrm{pH} 9.5$ is sufficient for stopping activity, as signal is stable after stopping. Inhibition assays were run in the presence or absence of various concentrations of inhibitors and at a fixed substrate concentration of $100 \mu \mathrm{M}$ and $1 \%$ DMSO. First, inhibitor was serially diluted to the desired range of concentrations in $2 \%$ DMSO buffer. Next, $25 \mu \mathrm{L}$ of inhibitor solutions at various concentrations in $2 \%$ DMSO buffer was added to $25 \mu \mathrm{L}$ of $4.0 \mathrm{nM}$ enzyme and allowed to incubate at $25{ }^{\circ} \mathrm{C}$ for 5 min. $50 \mu \mathrm{L}$ of $200 \mu \mathrm{M}$ and $1 \%$ DMSO substrate was then added and allowed to react for 20 min. $100 \mu \mathrm{L}$ of stop solution was added to reaction mixture, and immediately mixed and aliquoted in $45 \mu \mathrm{L}$ triplicates to a CORNING 384 well black plate. Fluorescence signal was measured in a BioTek Neo 2 Plate reader set at excitation and emission wavelengths of 355 and $450 \mathrm{~nm}$, respectively. \% Activity was subsequently calculated for each inhibitor concentration against the fluorescence signal of uninhibited HexA. GraphPad Prism 2016 was used to approximate the Morrison $K_{\mathrm{i}}$ values for each inhibitor.

Inhibition assays against rhHexB. Inhibition constant $\left(K_{\mathrm{i}}\right)$ values against recombinant human Hexosaminidase B (HexB), purchased from R\&D Systems (cat\# 8907-GH-020) are determined by measuring the change in fluorescent signal corresponding to the rate of hydrolytic activity against the commercially available artificial substrate, 4-methylumbelliferone N-acetyl- $\beta$-Dgalactosaminide. HexB activity assays were performed in $100 \mathrm{mM}$ MES buffer at pH 5.5 and $5 \mathrm{nM}[\mathrm{HexB}]$. Inhibition assays were in the presence or absence of various concentrations of inhibitors and at a fixed substrate concentration of $150 \mu \mathrm{M}$ and $1 \%$ DMSO. First, inhibitor was serially diluted to the desired range of concentrations in $2 \%$ DMSO buffer. Next, $50 \mu \mathrm{L}$ of inhibitor solutions at various concentrations in $2 \%$ DMSO Buffer was added to $50 \mu \mathrm{L}$ of $20 \mathrm{nM}$ enzyme and allowed to incubate at $25^{\circ} \mathrm{C}$ for $5 \mathrm{~min} .100 \mu \mathrm{L}$ of $300 \mu \mathrm{M}$ and $1 \%$ DMSO substrate was then added and reaction mixture was immediately mixed and aliquoted in $45 \mu \mathrm{L}$ triplicates to a CORNING 384 well black plate. Fluorescence signal was measured continuously for $20 \mathrm{~min}$ at $25{ }^{\circ} \mathrm{C}$ in a BioTek Neo 2 Plate reader set at excitation and emission wavelengths of 355 and $450 \mathrm{~nm}$, respectively. Max reaction rates for all inhibitor concentrations were calculated within Gen5 BioTek reader software. \% Activity was subsequently calculated for each inhibitor concentration against the max reaction rate of uninhibited HexB. GraphPad Prism 2016 was used to approximate the Morrison $K_{\mathrm{i}}$ values for each inhibitor.

Inhibition assays against BtGH84. The inhibition constant $\left(K_{\mathrm{i}}\right)$ value for compound 16 against recombinant OGA from Bacteriodes thetaiotaoicron ( $B t \mathrm{GH} 84$ ), which was produced and purified according to Dennis et al, 2006, was determined spectrophotometrically by measuring the change 
in absorbance at $405 \mathrm{~nm}$ upon hydrolysis of $p$-nitrophenyl $N$-acetyl- $\beta$-D-glucosaminide. A Biotek Epoch Microplate Spectrophotometer was used to monitor the enzyme assays that were performed in 384-well clear bottomed plates. The $K_{\mathrm{M}}$ value used in the tests was $1.09 \mathrm{mM}$ at pH $6.5(50 \mathrm{mM}$ MES pH 6.5, $200 \mathrm{mM} \mathrm{NaCl}$ ). The enzyme was incubated for $5 \mathrm{mins}$ at $25^{\circ} \mathrm{C}$ in the presence of various inhibitor concentrations after which the reaction was initiated by the addition of the substrate. The reaction was observed over 10 mins to achieve linear rates. $\mathrm{An} \mathrm{IC}_{50}$ was conducted using a fixed concentration of substrate (around the $K_{\mathrm{M}}$ value) to approximate the $K_{\mathrm{i}}$ value. Full $K_{\mathrm{i}}$ determinations were obtained from the slope of Lineweaver-Burk plots and taking the reciprocal of the slope on a plot of $\mathrm{K}_{\text {app }}$ vs the inhibitor concentration.

Cell culture. All cells were cultured at $37^{\circ} \mathrm{C}$ in a humidified incubator with $5 \% \mathrm{CO}_{2}$ using standard procedures. Briefly, SK-N-SH cells (ATCC) were cultured in EMEM (M5650, Sigma) supplemented with 10\% FBS (Gibco) and Pen/Strep antibiotics (Bioshop). Cells were expanded in T175 flasks and media was changed every 3 to 4 days until cells reached $80-90 \%$ confluency. For each passage, cells were washed with warm PBS, treated with the minimal volume of Trypsin/EDTA, incubated for 3 to 5 minutes until cells were fully detached, and re-suspended in media containing FBS. Cells were counted using BioRad Automated Cell Counter TC20 with Trypan blue (Gibco), and either split into a new flask for sub-culturing (1:4 to 1:5 dilution) or plated for treatments. All experiments were carried out with cells having a passage number between P13 and P18.

Cell plating and treatment. After counting, the concentration of cells was adjusted and $2.5 \times 10^{6}$ cells were plated in $10 \mathrm{~cm}$ petri dishes (TC Dish-100, Sarstedt). Dishes were incubated for 2 days before medium was exchanged and inhibitors diluted in medium (final concentrations of 0-3.2 $\mu \mathrm{M}$ for 33 and $1 \mu \mathrm{M}$ for Thiamet-G; $<0.1 \%$ DMSO content) were added. The treated cells were incubated for 2 days before harvesting.

Cell harvesting. Culture plates were put on ice, the medium was aspirated, and cold PBS $\left(4{ }^{\circ} \mathrm{C}\right.$, LonzaBioWhittaker) was added to all wells. The cells were scraped off and spun down at $300 \mathrm{~g}$ for 5 min at $4{ }^{\circ} \mathrm{C}$ (Sorvall Legend Micro 17R, Thermo Scientific). Cell pellets were resuspended in cold PBS buffer ( $3 \mathrm{x}$ the volume of the cell pellet) containing complete protease inhibitor cocktail tablets (Roche) prior to freeze-thaw-lysis. Samples were 3x plunged into liquid nitrogen and thawed in a water bath at $25{ }^{\circ} \mathrm{C}$ (280 series, Precision). Following, samples were spun down at $20,800 \mathrm{~g}$ at $4{ }^{\circ} \mathrm{C}$ for $30 \mathrm{~min}$ (centrifuge $5417 \mathrm{C}$, Eppendorf) and the supernatant was collected in fresh microcentrifuge tubes. The protein concentration of the samples was measured using the Quick Start Bradford 1x Dye Reagent (Biorad) and the Synergy neo2 plate reader (BioTek). 
Immunoblotting. Samples were diluted in 5x SDS-PAGE loading buffer and heated at $90{ }^{\circ} \mathrm{C}$ for 3 min. Following, samples were quickly spun down and separated in Mini-PROTEAN TGX gels (4-15\%, 10-well comb, $50 \mathrm{ul}$, Bio-Rad) at $70 \mathrm{~V}$ (PowerPac Basic, BioRad). Proteins were transferred to a $0.45 \mu \mathrm{m}$ nitrocellulose membrane (Bio-Rad) by wet transfer at $110 \mathrm{~V}$ at $4{ }^{\circ} \mathrm{C}$ for $70 \mathrm{~min}$. The membrane was incubated with $4 \%$ BSA (BioShop) in PBS buffer at $4{ }^{\circ} \mathrm{C}$ for 1 hour prior to incubation overnight $\left(4^{\circ} \mathrm{C}\right)$ on a rocking platform (Scilogex) with primary antibodies for fluorescent immunoblotting. Following primary antibodies were used: anti-OGA (1:5,000, rabbit, SAB4200311, Sigma-Aldrich), anti-O-GlcNAc clone CTD110.6 (1:3,000, mouse, MABS1254, EMD Millipore), and $\beta$-anti-Actin (1:10,000, rabbit, 926-42210, Li-Cor). On the following day, the membrane was washed $3 \mathrm{x}$ with PBS-T (0.1\% tween 20 , BioShop) for each $2 \mathrm{~min}$. The membrane was then incubated with the secondary antibodies in 2\% BSA in PBS-T at RT for 1 hour. Following secondary antibodies were used: Goat-anti-rabbit IRDye 800CW (1:20,000, 92632211, Li-Cor) and Goat-anti-mouse IRDye 680LT (1:20,000, 926-68020, Li-Cor). Membranes were then washed $3 \mathrm{x}$ with PBS-T for each 2 min prior to imaging (Odyssey, Li-Cor).

Expression and purification of recombinant hOGA. E. coli cells that were transformed with a plasmid expressing hexahistidine tagged hOGA (full-length) were inoculated in lysogeny broth (LB) medium containing kanamycin $(50 \mu \mathrm{g} / \mathrm{mL})$, and were incubated aerobically at $37{ }^{\circ} \mathrm{C}$ overnight. The following day, $10 \mathrm{~L}$ of LB containing kanamycin $(50 \mu \mathrm{g} / \mathrm{mL})$, were inoculated with the overnight culture (1:100) and incubated aerobically at $37^{\circ} \mathrm{C}$ to an OD600 of $\sim 1.0$. Once reached, cells were cold shocked at $4{ }^{\circ} \mathrm{C}$ for $30 \mathrm{~min}$, then had IPTG added $(0.1 \mathrm{mM})$ to induce protein synthesis, and expression induced at $21^{\circ} \mathrm{C}$ overnight. The cells were harvested by centrifugation (high-speed) at 20,000 RPM at $4{ }^{\circ} \mathrm{C}$ for $20 \mathrm{~min}$, and cells were flash frozen in liquid nitrogen, and stored at $-80^{\circ} \mathrm{C}$ until needed.

For purification of hOGA, cells were thawed and resuspended in $25 \mathrm{mM}$ HEPES, $500 \mathrm{mM} \mathrm{NaCl}$, $1 \mathrm{mM}$ DTT, pH 7.0, $5 \mathrm{mM}$ imidazole (resuspension buffer), and rocked with lysozyme (1 mg/mL) at $4{ }^{\circ} \mathrm{C}$ for $30 \mathrm{~min}$. Cells were lysed using a sonic dismembrator at amplitude $60 \%$, for $3 \mathrm{~min}(20$ $\mathrm{sec}$ on, $40 \mathrm{sec}$ off). The lysate was cleared by centrifugation (high-speed) at 20,000 RPM at $4{ }^{\circ} \mathrm{C}$ for $30 \mathrm{~min}$, and again (ultra-speed) at $45,000 \mathrm{RPM}$ at $4{ }^{\circ} \mathrm{C}$ for $40 \mathrm{~min}$. The supernatant was passed through a pre-equilibrated HisTrap FF column (GE Healthcare) in suspension buffer using a peristaltic pump (flow rate $2 \mathrm{~mL} / \mathrm{min}$ ). The column loaded with hOGA was purified by a gradient of $0 \%$ to $50 \%$ of elution buffer ( $25 \mathrm{mM}$ HEPES, $500 \mathrm{mM} \mathrm{NaCl}, 1 \mathrm{mM}$ DTT, $\mathrm{pH} 7.0$, and 500 $\mathrm{mM}$ imidazole) over $50 \mathrm{~mL}$ of volume. Fractions containing hOGA were pooled and dialyzed using a molecular weight cut-off (MWCO) of $30 \mathrm{kDa}$ in final buffer $(10 \mathrm{mM}$ HEPES, $250 \mathrm{mM}$ $\mathrm{NaCl}, 1 \mathrm{mM}$ DTT, pH 7.0). Dialyzed hOGA was concentrated using a Vivaspin of $70 \mathrm{kDa}$ 
MWCO, and had concentrations determined by Nanodrop 2000 UV-Vis spectrophotometer. Purified hOGA was aliquoted, flash frozen in liquid nitrogen, and stored at $-80{ }^{\circ} \mathrm{C}$ until required.

Western blotting. Samples were diluted in $5 \mathrm{x}$ SDS-PAGE loading buffer and heated at $90^{\circ} \mathrm{C}$ for 3 min. Following, samples were quickly spun down and separated in Mini-PROTEAN TGX gels (4-15\%, 10-well comb, $50 \mu 1$, Bio-Rad) at $70 \mathrm{~V}$ (PowerPac Basic, BioRad). Proteins were transferred to a $0.45 \mu \mathrm{m}$ nitrocellulose membrane (Bio-Rad) by wet transfer at $110 \mathrm{~V}$ at $4{ }^{\circ} \mathrm{C}$ for $70 \mathrm{~min}$. The membrane was incubated with 4\% BSA (BioShop) in PBS buffer at $4{ }^{\circ} \mathrm{C}$ for 1 hour prior to incubation overnight $\left(4{ }^{\circ} \mathrm{C}\right)$ on a rocking platform (Scilogex) with primary antibodies for fluorescent immunoblotting. Following primary antibodies were used: anti-OGA (1:5,000, rabbit, SAB4200311, Sigma-Aldrich), anti-O-GlcNAc clone CTD110.6 (1:3,000, mouse, MABS1254, EMD Millipore), and $\beta$-anti-Actin (1:10,000, rabbit, 926-42210, Li-Cor). On the following day, the membrane was washed $3 \mathrm{x}$ with PBS-T $(0.1 \%$ tween 20 , BioShop) for each 2 min. The membrane was then incubated with the secondary antibodies in 2\% BSA in PBS-T at RT for 1 hour. Following secondary antibodies were used: Goat-anti-rabbit IRDye 800CW (1:20,000, 92632211, Li-Cor) and Goat-anti-mouse IRDye 680LT (1:20,000, 926-68020, Li-Cor). Membranes were then washed $3 \mathrm{x}$ with PBS-T for each 2 min prior to imaging (Odyssey, Li-Cor).

\section{Materials}

2-Acetamido-1,2-dideoxynojirimicin hydrochloride (DNJNAc $\mathrm{HCl}$ ), 2-azido-3,6-di-O-acetyl-4$O$-benzyl-1,2,5-trideoxy-1,5-imino-D-glucitol (25), ${ }^{1} \quad$ 1,4-bis(azidomethyl)benzene ${ }^{2}$ and 1 naphtylmethyl isothiocyanate ${ }^{3}$ were prepared according to literature procedures. 1,5-Imino-2propamido-1,2,5-trideoxy-D-glucitol $(\mathbf{3 4})^{4}$ was previously characterized. $o-, m$ - and $p$ Azidomethylbenzylamines were obtained by cotrolled reduction of the corresponding $o-, m$ - and $p$-bis(azidomethyl)benzene derivatives with triphenyphosphine, following the method reported by Lau et al. ${ }^{5}$ They were used in the next isothiocyanation reaction without further purification. The non-commercial $p$-trifluoromethylbenzyl, $o$-, $m$-and $p$-azidomethylbenzyl and 5-indolyl isothiocyanates were synthesized by isothiocyanation of the corresponding amines as detailed hereinafter: 


\section{Synthesis of $p$-Trifluoromethylbenzyl isothiocyanate.}

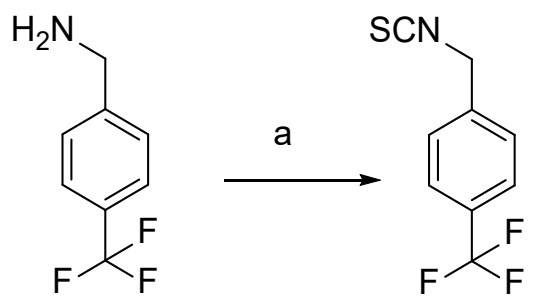

Supplemental scheme 1. Reagents and conditions: a. $\mathrm{CS}_{2}, \mathrm{Et}_{3} \mathrm{~N}$, Boc $2 \mathrm{O}, \mathrm{DMAP}, \mathrm{EtOH}, \mathrm{RT}, 18$ h.

To a solution of $p$-(trifluoromethyl)benzylamine $(213 \mu \mathrm{L}, 1.494 \mathrm{mM})$ in EtOH $(8 \mathrm{~mL}), \mathrm{CS}_{2}(928$ $\mu \mathrm{L}, 14.940 \mathrm{mM})$ and $\mathrm{Et}_{3} \mathrm{~N}(414 \mu \mathrm{L}, 2.99 \mathrm{mM})$ were added. The mixture was stirred at RT for 30 min. Then di-tert-butyl dicarbonate $(326 \mathrm{mg}, 1.49 \mathrm{mmol})$ and 4-dimethylaminopyridine $(3.7 \mathrm{mg}$, $0.029 \mathrm{mM}$ ) were added at $0{ }^{\circ} \mathrm{C}$ and the reaction mixture was stirred for $18 \mathrm{~h}$ at RT. The solvent was evaporated under reduced pressure and the residue was dissolved with DCM $(20 \mathrm{~mL})$, washed with water $(20 \mathrm{~mL})$, brine $(20 \mathrm{~mL})$, dried $\left(\mathrm{MgSO}_{4}\right)$ and concentrated. The crude product was purified by column chromatography $\left(1: 8 \rightarrow 1: 3\right.$ EtOAc- cyclohexane). Yield: $128 \mathrm{mg}(60 \%)$. $\mathrm{R}_{f}$ 0.66 (1:3 EtOAc-cyclohexane). $[\alpha]_{\mathrm{D}}+5.6$ (c 1.0 in DCM).

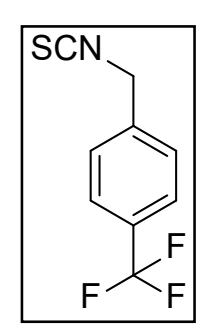

${ }^{1} \mathbf{H}$ NMR $\left(300 \mathrm{MHz}, \mathrm{CDCl}_{3}\right): \delta=7.68\left(\mathrm{~d}, 1 \mathrm{H},{ }^{3} J_{\mathrm{H}, \mathrm{H}}=8.1 \mathrm{~Hz}, \mathrm{CH}_{\text {arom }}\right), 7.47(\mathrm{~d}$, $1 \mathrm{H}, \mathrm{CH}_{\text {arom }}$ ), 4.81 (s, $\left.2 \mathrm{H}, \mathrm{CH}_{2} \mathrm{NCS}\right) .{ }^{13} \mathbf{C}$ NMR $\left(100.6 \mathrm{MHz}, \mathrm{CDCl}_{3}\right): \delta=138.2$ (C-1), 130.9 (C-4), 127.0 (C-2), 126.0 ( $\left.J_{\mathrm{C}, \mathrm{F}}=3.7 \mathrm{~Hz}, \mathrm{CF}_{3}, \mathrm{C}-3\right), 122.0$ (NCS). ESIMS: $m / z 215.8$ [M - H] $]^{-}$Anal. Calcd. for $\mathrm{C}_{9} \mathrm{H}_{6} \mathrm{~F}_{3} \mathrm{NS}$ : C 49.77, H 2.78, F 26.24, N 6.45, S 14.76. Found C 49.63, H 2.56, N 6.27, S 14.49. 
Synthesis of $o-, m$ - and $p$-Azidomethylbenzyl isothiocyanates.

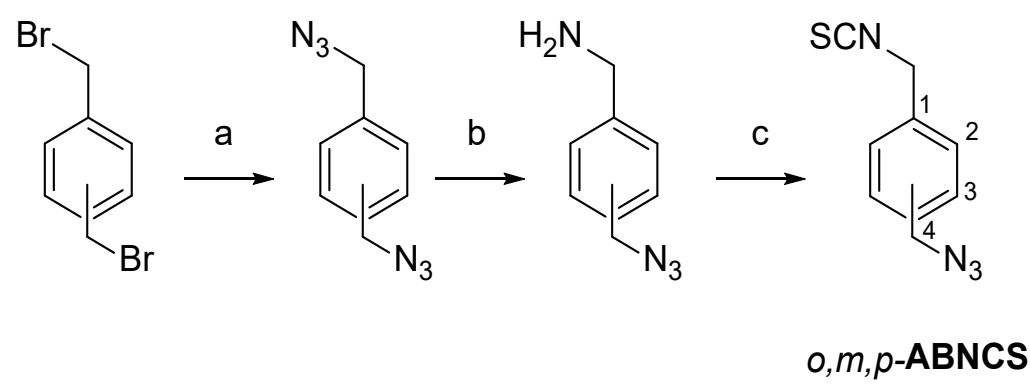

Supplemental scheme 2. Reagents and conditions: a. $\mathrm{NaN}_{3}$, DMF, $60{ }^{\circ} \mathrm{C}, 18 \mathrm{~h}$; b. $\mathrm{PPh}_{3}, \mathrm{HCl} 1 \mathrm{M}$ $\mathrm{Et}_{2} \mathrm{O}, \mathrm{RT}, 24$ h; c. $\mathrm{CS}_{2}, \mathrm{Cu}_{2} \mathrm{SO}_{4} \cdot 5 \mathrm{H}_{2} \mathrm{O}, \mathrm{Et}_{3} \mathrm{~N}, 2: 1 \mathrm{EtOAc}-\mathrm{H}_{2} \mathrm{O}, \mathrm{RT}, 18 \mathrm{~h}$.

General procedure for $\boldsymbol{o , m}, \boldsymbol{p}$-ABNCS synthesis. To a solution of the corresponding $o-, m$ - or $p$-azidomethylbenzylamine $(1.0 \mathrm{~g}, 6.5 \mathrm{mM})$ in EtOAc- $\mathrm{H}_{2} \mathrm{O}(2: 1,18 \mathrm{~mL}), \mathrm{CS}_{2}(3.9 \mathrm{~mL}, 65 \mathrm{mM})$ and $\mathrm{Et}_{3} \mathrm{~N}(1.8 \mathrm{~mL}, 13 \mathrm{mM})$ were added and the mixture was stirred at r.t. for $1 \mathrm{~h}$. Then, $\mathrm{Cu}_{2} \mathrm{SO}_{4} \cdot 5 \mathrm{H}_{2} \mathrm{O}(413 \mathrm{mg}, 1.65 \mathrm{mM})$ was added and the mixture was further stirred at RT for $16 \mathrm{~h}$. The crude product was filtered, diluted with EtOAc $(20 \mathrm{~mL})$, washed with $\mathrm{H}_{2} \mathrm{O}(20 \mathrm{~mL})$, dried $\left(\mathrm{MgSO}_{4}\right)$ and concentrated. The crude was purified by column chromatography (1:10 EtOAccyclohexane).

o-(Azidomethyl)benzyl isothiocyanate (o-ABNCS). Yield: $0.97 \mathrm{~g}(72 \%) . \mathrm{R}_{f} 0.51$ (1:8 EtOAccyclohexane). $[\alpha]_{\mathrm{D}}-1.62$ (c 1.00 in DCM).

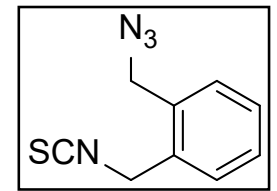

${ }^{1} \mathbf{H}$ NMR (300 MHz, $\left.\mathrm{CDCl}_{3}\right): \delta$ 7.49-7.33 (m, $\left.4 \mathrm{H}, \mathrm{CH}_{\text {arom }}\right), 4.82(\mathrm{~s}, 2 \mathrm{H}$, $\left.\mathrm{CH}_{2} \mathrm{NCS}\right), 4.42\left(\mathrm{~s}, 2 \mathrm{H}, \mathrm{CH}_{2} \mathrm{~N}_{3}\right) .{ }^{13} \mathbf{C}$ NMR $\left(75.5 \mathrm{MHz}, \mathrm{CDCl}_{3}\right): \delta=133.2$ (NCS), 132.9 (C-1), 132.8 (C-6), 130.2 (C-5), 129.4 (C-2), 129.0 (C-3), 128.8 (C-4), $52.4\left(\mathrm{CH}_{2} \mathrm{NCS}\right), 46.3\left(\mathrm{CH}_{2} \mathrm{~N}_{3}\right)$. ESIMS: $\mathrm{m} / z 215.8$ [M - H] ${ }^{-}$. Anal. Calcd. for $\mathrm{C}_{9} \mathrm{H}_{8} \mathrm{~N}_{4} \mathrm{~S}: \mathrm{C}$ 52.92, H 3.95, N 27.43, S 15.70. Found C 53.23, H 4.21, N 27.31, S 15.48.

$\boldsymbol{m}$-(Azidomethyl)benzyl isothiocyanate. Yield: $0.92 \mathrm{~g}(68 \%) . \mathrm{R}_{f} 0.51$ (1:8 EtOAc-cyclohexane). $[\alpha]_{\mathrm{D}}-0.71(c 1.00$ in DCM).

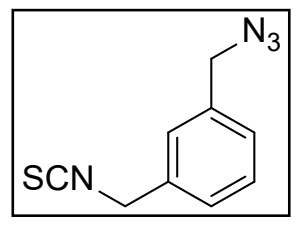

${ }^{1} \mathbf{H}$ NMR (300 MHz, $\left.\mathrm{CDCl}_{3}\right): \delta$ 7.46-7.28 (m, $\left.4 \mathrm{H}, \mathrm{CH}_{\text {arom }}\right), 4.76(\mathrm{~s}, 2 \mathrm{H}$, $\left.\mathrm{CH}_{2} \mathrm{NCS}\right), 4.40\left(\mathrm{~s}, 2 \mathrm{H}, \mathrm{CH}_{2} \mathrm{~N}_{3}\right) .{ }^{13} \mathrm{C}$ NMR $\left(75.5 \mathrm{MHz}, \mathrm{CDCl}_{3}\right): \delta=136.4$ (C-1), 135.0 (C-5), 132.8 (NCS), 129.5 (C-2), 128.1 (C-3), 126.7 (C-4), $126.4(\mathrm{C}-6), 54.4\left(\mathrm{CH}_{2} \mathrm{NCS}\right), 48.4\left(\mathrm{CH}_{2} \mathrm{~N}_{3}\right)$. ESIMS: $m / z=215.8[\mathrm{M} \mathrm{-} \mathrm{H}]^{-}$ . Anal. Calcd. for $\mathrm{C}_{9} \mathrm{H}_{8} \mathrm{~N}_{4} \mathrm{~S}$ : C 52.92, H 3.95, N 27.43, S 15.70. Found C 53.18, H 4.18, N 27.26, S 15.44 . 
p-(Azidomethyl)benzyl isothiocyanate. Yield: $1.0 \mathrm{~g}(74 \%) ; \mathrm{R}_{f} 0.51$ (1:8 EtOAc-cyclohexane). $[\alpha]_{\mathrm{D}}-2.51$ (c 1.00 in DCM).

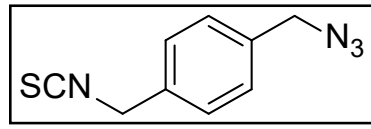

${ }^{1} \mathbf{H}$ NMR (300 MHz, $\left.\mathrm{CDCl}_{3}\right): \delta 7.36\left(\mathrm{~s}, 4 \mathrm{H}, \mathrm{CH}_{\text {arom }}\right), 4.74(\mathrm{~s}, 2 \mathrm{H}$, $\left.\mathrm{CH}_{2} \mathrm{NCS}\right), 4.37\left(\mathrm{~s}, 2 \mathrm{H}, \mathrm{CH}_{2} \mathrm{~N}_{3}\right) .{ }^{13} \mathbf{C}$ NMR $\left(100.6 \mathrm{MHz}, \mathrm{CDCl}_{3}\right): \delta=$ 135.7 (C-1), 134.3 (C-4), 129.7 (NCS), 128.7 (C-2), 127.3 (C-3), $54.3\left(\mathrm{CH}_{2} \mathrm{NCS}\right), 48.3\left(\mathrm{CH}_{2} \mathrm{~N}_{3}\right)$. ESIMS: $m / z=215.8[\mathrm{M}-\mathrm{H}]^{-}$. Anal. calcd. for $\mathrm{C}_{9} \mathrm{H}_{8} \mathrm{~N}_{4} \mathrm{~S}$ : C 52.92, H 3.95, N 27.43, S 15.70. Found C 53.04, H 3.99, N 27.17, S 15.39.

\section{Synthesis of tert-butyl 5-(isothiocyanatomethyl)-1H-indole-1-carboxylate.}<smiles>CC(C)(C)C(C)(C)C(C)(C)C</smiles>

Supplemental scheme 3. Reagents and conditions: a. $\mathrm{CS}_{2}, \mathrm{Et}_{3} \mathrm{~N}$, Boc $2 \mathrm{O}, \mathrm{DMAP}, \mathrm{EtOH}, \mathrm{RT}, 18$ h; b. $\mathrm{Boc}_{2} \mathrm{O}, \mathrm{Et}_{3} \mathrm{~N}, \mathrm{DCM}, \mathrm{RT}, 18 \mathrm{~h}$.

To a solution of (1H-indol-5-yl)methanamine $(600 \mathrm{mg}, 2 \mathrm{mM})$ in EtOH $(22 \mathrm{~mL}), \mathrm{CS}_{2}(2.4 \mathrm{~mL}$, $20 \mathrm{mM})$ and $\mathrm{Et}_{3} \mathrm{~N}(1 \mathrm{~mL}, 4 \mathrm{mM})$ were added. The mixture was stirred at RT for $30 \mathrm{~min}$. Then ditert-butyl dicarbonate $(875 \mathrm{mg}, 2 \mathrm{mM})$ and 4-dimethylaminopyridine (10 $\mathrm{mg}, 0.04 \mathrm{mM})$ were added at $0{ }^{\circ} \mathrm{C}$ and the reaction mixture was stirred for $18 \mathrm{~h}$ at $\mathrm{RT}$. The solvent was evaporated under reduced pressure and the residue was dissolved with DCM $(30 \mathrm{~mL})$, washed with water $(30$ $\mathrm{mL})$, brine $(30 \mathrm{~mL})$, dried $\left(\mathrm{MgSO}_{4}\right)$ and concentrated. The crude product was directly dissolved in $\mathrm{DCM}(15 \mathrm{~mL})$ and $\mathrm{Et}_{3} \mathrm{~N}(0.5 \mathrm{~mL})$ and $\mathrm{Boc}_{2} \mathrm{O}(800 \mathrm{mg})$ were added and the mixture was stirred for $18 \mathrm{~h}$ at RT. The solvent was evaporated and the crude product purified by column chromatography (1:4 EtOAc-cyclohexane). Yield: $300 \mathrm{mg}$ (50\%, over 2 steps). $\mathrm{R}_{f} 0.50$ (1:4 EtOAc-cyclohexane).

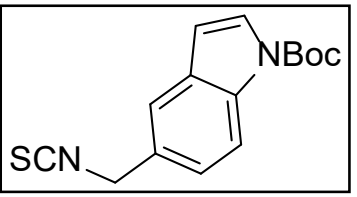

${ }^{1}$ H NMR (300 MHz, $\left.\mathrm{CDCl}_{3}\right): \delta 8.08(\mathrm{~d}, 1 \mathrm{H}, \mathrm{H}-4), 7.55$ (d, $\left.1 \mathrm{H}, \mathrm{H}-7\right)$, 7.43 (s, $1 \mathrm{H}, \mathrm{H}-6), 7.10$ (dd, $1 \mathrm{H}, \mathrm{H}-1), 6.40$ (d, $1 \mathrm{H}, \mathrm{H}-2), 4.71$ (s, $2 \mathrm{H}$, $\left.\mathrm{CH}_{2} \mathrm{NCS}\right), 1.60\left(\mathrm{~s}, 9 \mathrm{H}, \mathrm{C}\left(\mathrm{CH}_{3}\right)_{3}\right) .{ }^{13} \mathbf{C} \mathbf{N M R}\left(75.5 \mathrm{MHz}, \mathrm{CDCl}_{3}\right): \delta=$

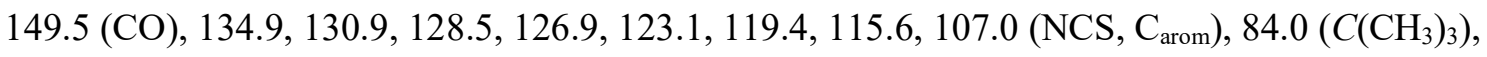
$48.9\left(\mathrm{CH}_{2} \mathrm{NCS}\right), 28.1\left(\mathrm{C}\left(\mathrm{CH}_{3}\right)_{3}\right)$. ESIMS: $m / z=287.40[\mathrm{M}-\mathrm{H}]^{-}$.

\section{Synthesis of DNJNAc-thioureas 1-12}




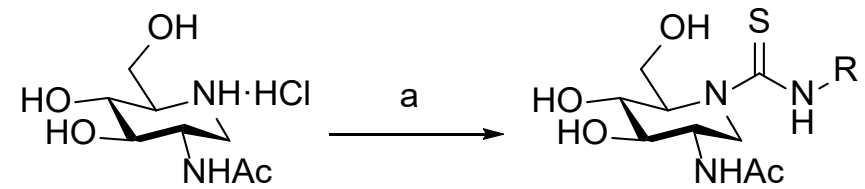

Supplemental scheme 4. Reagents and conditions: a. Et $t_{3}$, R-NCS, DMF, RT, $18 \mathrm{~h}$.

General procedure for the preparation of the DNJNAc-thioureas 1-12. To a solution of 2- $\mathrm{N}$ acetamido-1,2-dideoxynojirimicin hydrochloride $(100 \mathrm{mg}, 0.415 \mathrm{mM})$ in DMF $(3.7 \mathrm{~mL}), \mathrm{Et}_{3} \mathrm{~N}$ $(0.115 \mathrm{~mL}, 0.830 \mathrm{mM})$ and the corresponding isothiocyanate $(0.498 \mathrm{mM}, 1.2 \mathrm{eq})$ were added. The mixture was stirred at RT for $18 \mathrm{~h}$. Then, the solvent was coevapored with toluene and the resulting residue was purified by column chromatography using the eluent indicated in each case.

2-Acetamido-5- $N$-(N'-butylthiocarbamoyl)-1,2-dideoxynojirimycin

(1). Column chromatography, eluent 80:10:1 DCM-MeOH-H $\mathrm{H}_{2} \mathrm{O}$. Yield: $116 \mathrm{mg}(86 \%)$. [ $\left.\alpha\right]_{\mathrm{D}}-114.7$ (c 1.04, $\mathrm{MeOH}) . \mathrm{R}_{f} 0.35$ (70:10:1 DCM-MeOH-H$\left.{ }_{2} \mathrm{O}\right)$. UV (MeOH) $249 \mathrm{~nm}\left(\varepsilon_{\mathrm{mm}} 12.5\right)$.

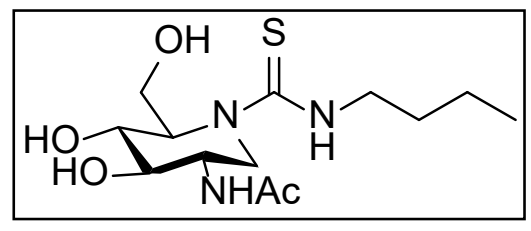

${ }^{1} \mathbf{H}$ NMR (500 MHz, CD 3 OD): $\delta 4.83\left(\mathrm{bd}, 1 \mathrm{H}, J_{1 \mathrm{a}, 1 \mathrm{~b}}=14.4\right.$ Hz, H-1a), 4.25 (m, $1 \mathrm{H}, \mathrm{H}-5), 3.92\left(\mathrm{dd}, 1 \mathrm{H}, J_{5,6 \mathrm{a}}=8.6 \mathrm{~Hz}\right.$, $\left.J_{6 \mathrm{a}, 6 \mathrm{~b}}=11.4 \mathrm{~Hz}, \mathrm{H}-6 \mathrm{a}\right), 3.90(\mathrm{~m}, 1 \mathrm{H}, \mathrm{H}-2), 3.84$ (dd, $1 \mathrm{H}, J_{5,6 \mathrm{~b}}$ $=3.6 \mathrm{~Hz}, \mathrm{H}-6 \mathrm{~b}), 3.67\left(\mathrm{dd}, 1 \mathrm{H}, J_{3,4}=6.1 \mathrm{~Hz}, J_{4,5}=4.7 \mathrm{~Hz}, \mathrm{H}-\right.$

4), 3.57 (m, $\left.2 \mathrm{H}, \mathrm{CH}_{2} \mathrm{NH}, \mathrm{H}-3\right), 3.47$ (dd, $\left.1 \mathrm{H}, J_{1 \mathrm{~b}, 2}=4.0 \mathrm{~Hz}, \mathrm{H}-1 \mathrm{~b}\right), 1.95$ (s, $\left.1 \mathrm{H}, \mathrm{COCH}_{3}\right), 1.59$ (m, $\left.2 \mathrm{H}, \mathrm{CH}_{2}\right), 1.38\left(\mathrm{~m}, 2 \mathrm{H}, \mathrm{CH}_{2} \mathrm{CH}_{3}\right), 0.95\left(\mathrm{t}, 3 \mathrm{H}, \mathrm{J}_{\mathrm{H}, \mathrm{H}}=7.4 \mathrm{~Hz}, \mathrm{CH}_{3}\right) .{ }^{13} \mathbf{C} \mathbf{N M R}(125.7 \mathrm{MHz}$, $\mathrm{CD}_{3} \mathrm{OD}$ ): $\delta 186.6$ (CS), $173.0(\mathrm{CO}), 72.8$ (C-3), 70.8 (C-4), 66.2 (C-5), 62.2 (C-6), 53.3 (C-2), $46.8\left(\mathrm{CH}_{2} \mathrm{~N}\right), 45.2(\mathrm{C}-1), 32.3\left(\mathrm{CH}_{2}\right), 23.0\left(\mathrm{COCH}_{3}\right), 21.1\left(\mathrm{CH}_{2} \mathrm{CH}_{3}\right), 14.1\left(\mathrm{CH}_{3}\right)$. CIMS: $m / z 320$ $\left(10,[\mathrm{M}+\mathrm{H}]^{+}\right)$. Anal. Calcd for $\mathrm{C}_{13} \mathrm{H}_{25} \mathrm{~N}_{3} \mathrm{O}_{4} \mathrm{~S}: \mathrm{C}, 48.88 ; \mathrm{H}, 7.89 ; \mathrm{N}, 13.16 ; \mathrm{S}, 10.04$. Found: $\mathrm{C}$, 48.72; H, 7.69; N, 12.83; S, 9.69.

\section{2-Acetamido-1,2-dideoxy-5-N-(N'-octylthiocarbamoyl)nojirimycin}

(2). Column chromatography, eluent 80:10:1 DCM-MeOH- $\mathrm{H}_{2} \mathrm{O}$. Yield: $124 \mathrm{mg}(78 \%)$. $[\alpha]_{\mathrm{D}}-73.8(c$ 1.00, $\mathrm{MeOH}) . \mathrm{R}_{f} 0.39$ (70:10:1 DCM-MeOH-H $\left.{ }_{2} \mathrm{O}\right)$. UV (MeOH) $248 \mathrm{~nm}\left(\varepsilon_{\mathrm{mm}} 11.4\right)$.

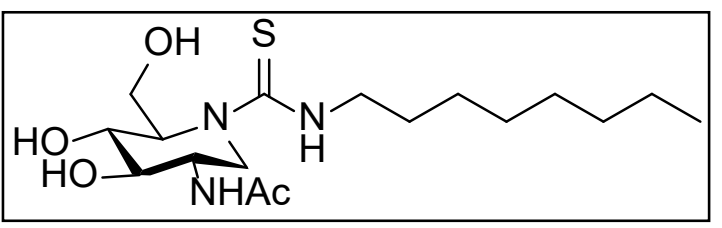

${ }^{1} \mathbf{H}$ NMR (500 MHz, CD $\left.3 \mathrm{OD}\right): \delta 4.83(\mathrm{bd}, 1 \mathrm{H}$, $\left.J_{1 \mathrm{a}, 1 \mathrm{~b}}=14.6 \mathrm{~Hz}, \mathrm{H}-1 \mathrm{a}\right), 4.23$ (m, $\left.1 \mathrm{H}, \mathrm{H}-5\right), 3.92$ $\left(\mathrm{dd}, 1 \mathrm{H}, J_{6 \mathrm{a}, 6 \mathrm{~b}}=11.4 \mathrm{~Hz}, J_{5,6 \mathrm{a}}=8.8 \mathrm{~Hz}, \mathrm{H}-6 \mathrm{a}\right)$,

3.88 (m, $1 \mathrm{H}, \mathrm{H}-2), 3.83$ (dd, $\left.1 \mathrm{H}, J_{5,6 \mathrm{~b}}=3.5 \mathrm{~Hz}, \mathrm{H}-6 \mathrm{~b}\right), 3.66$ (t, $\left.1 \mathrm{H}, J_{3,4}=J_{4,5}=6.0 \mathrm{~Hz}, \mathrm{H}-4\right), 3.56$ (m, $\left.2 \mathrm{H}, \mathrm{CH}_{2} \mathrm{NH}, \mathrm{H}-3\right), 3.46\left(\mathrm{dd}, 1 \mathrm{H}, J_{1 \mathrm{~b}, 2}=4.1 \mathrm{~Hz}, \mathrm{H}-1 \mathrm{~b}\right), 1.94\left(\mathrm{~s}, 1 \mathrm{H}, \mathrm{COCH}_{3}\right), 1.60$ (m, $2 \mathrm{H}$, $\left.\mathrm{CH}_{2}\right), 1.31\left(\mathrm{~m}, 10 \mathrm{H}, \mathrm{CH}_{2}\right), 0.90\left(\mathrm{t}, 3 \mathrm{H},{ }^{3} J_{\mathrm{H}, \mathrm{H}}=7.0 \mathrm{~Hz}, \mathrm{CH}_{3}\right) .{ }^{13} \mathbf{C ~ N M R}\left(125.7 \mathrm{MHz}, \mathrm{CD}_{3} \mathrm{OD}\right): \delta$ $186.6(\mathrm{CS}), 173.0(\mathrm{CO}), 72.8$ (C-3), $70.8(\mathrm{C}-4), 66.2(\mathrm{C}-5), 62.1(\mathrm{C}-6), 53.2(\mathrm{C}-2), 47.1\left(\mathrm{CH}_{2} \mathrm{~N}\right)$, 45.2 (C-1), 32.9, 30.4, 30.3, 30.1 28.0, $23.6\left(\mathrm{CH}_{2}\right), 23.0\left(\mathrm{COCH}_{3}\right), 14.3\left(\mathrm{CH}_{3}\right)$. ESIMS: $\mathrm{m} / z 376$ 
$[\mathrm{M}+\mathrm{H}]^{+}, 398[\mathrm{M}+\mathrm{Na}]^{+}$. Anal. Calcd for $\mathrm{C}_{17} \mathrm{H}_{33} \mathrm{~N}_{3} \mathrm{O}_{4} \mathrm{~S}: \mathrm{C}, 54.37 ; \mathrm{H}, 8.86 ; \mathrm{N}, 11.19 ; \mathrm{S}, 8.54$. Found: C, 54.51; H, 8.91; N, 10.94; S 8.22.

2-Acetamido-1,2-dideoxy-5-N-(N'-phenylthiocarbamoyl)nojirimycin

(3). Column

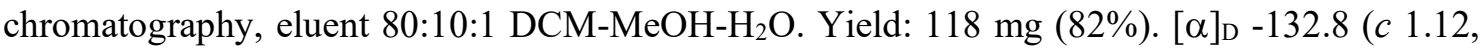
$\mathrm{MeOH}) . \mathrm{R}_{f} 0.29$ (70:10:1 DCM-MeOH-H $\left.{ }_{2} \mathrm{O}\right)$. UV (MeOH) $258 \mathrm{~nm}\left(\varepsilon_{\mathrm{mm}} 14.9\right)$.

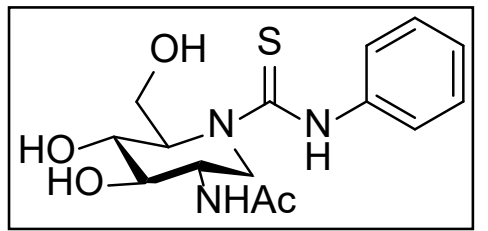

${ }^{1} \mathbf{H}$ NMR $\left(400 \mathrm{MHz}\right.$, acetone- $\left.d_{6}\right): \delta 9.83(\mathrm{bs}, 1 \mathrm{H}, \mathrm{NH}), 7.88$ (bd, $\left.1 \mathrm{H}, J_{\mathrm{NH}, 2}=6.4 \mathrm{~Hz}, \mathrm{NH}\right), 7.67-7.08\left(\mathrm{~m}, 5 \mathrm{H}, \mathrm{CH}_{\text {arom }}\right), 5.45$ (bs, $1 \mathrm{H}, \mathrm{OH}), 5.03$ (bs, $1 \mathrm{H}, \mathrm{OH}), 4.91\left(\mathrm{~d}, 1 \mathrm{H}, J_{1 \mathrm{a}, 1 \mathrm{~b}}=14.4 \mathrm{~Hz}\right.$, H-1a), 4.62 (bs, $1 \mathrm{H}, \mathrm{OH}), 4.40$ (m, $1 \mathrm{H}, \mathrm{H}-5), 4.13$ (t, $1 \mathrm{H}, J_{5,6 \mathrm{a}}$

$\left.=J_{6 \mathrm{a}, 6 \mathrm{~b}}=11.1 \mathrm{~Hz}, \mathrm{H}-6 \mathrm{a}\right), 4.04\left(\mathrm{dd}, 1 \mathrm{H}, J_{5,6 \mathrm{~b}}=2.9 \mathrm{~Hz}, \mathrm{H}-6 \mathrm{~b}\right), 3.92(\mathrm{~m}, 1 \mathrm{H}, \mathrm{H}-2), 3.75\left(\mathrm{t}, 1 \mathrm{H}, J_{3,4}\right.$ $\left.=J_{4,5}=6.8 \mathrm{~Hz}, \mathrm{H}-4\right), 3.63\left(\mathrm{dd}, 1 \mathrm{H}, J_{2,3}=4.7 \mathrm{~Hz}, \mathrm{H}-3\right), 3.57$ (dd, $\left.1 \mathrm{H}, J_{1 \mathrm{~b}, 2}=4.4 \mathrm{~Hz}, \mathrm{H}-1 \mathrm{~b}\right), 1.93$ (s, $\left.1 \mathrm{H}, \mathrm{COCH}_{3}\right) .{ }^{13} \mathbf{C} \mathbf{~ N M R}\left(100.6 \mathrm{MHz}\right.$, acetone- $\left.d_{6}\right): \delta 185.8(\mathrm{CS}), 171.2(\mathrm{CO}), 141.8,129.0$, 124.9, 124.2 ( $\mathrm{C}_{\text {arom}}$ ), 74.6 (C-3), 70.5 (C-4), 66.6 (C-5), 62.7 (C-6), 54.4 (C-2), 44.5 (C-1), 23.2 $\left(\mathrm{COCH}_{3}\right)$. ESIMS: $m / z 362[\mathrm{M}+\mathrm{Na}]^{+}$. Anal. Calcd for $\mathrm{C}_{15} \mathrm{H}_{21} \mathrm{~N}_{3} \mathrm{O}_{4} \mathrm{~S}: \mathrm{C}, 53.08 ; \mathrm{H}, 6.24 ; \mathrm{N}, 12.38$; S, 9.45. Found: C, 53.20; H, 6.28; N, 12.41; S 9.60.

2-Acetamido-5- $N$-(N'-benzylthiocarbamoyl)-1,2-dideoxynojirimycin $\quad$ (4). Column chromatography, eluent 80:10:1 DCM-MeOH-H ${ }_{2} \mathrm{O}$. Yield: $117 \mathrm{mg}(78 \%)$. [ $\left.\alpha\right]_{\mathrm{D}}-48.7$ (c 0.46, $\mathrm{MeOH}) . \mathrm{R}_{f}$ 0.33 (70:10:1 DCM-MeOH-H $\left.2 \mathrm{O}\right)$. UV (MeOH) $208 \mathrm{~nm}\left(\varepsilon_{\mathrm{mm}} 18.0\right)$.

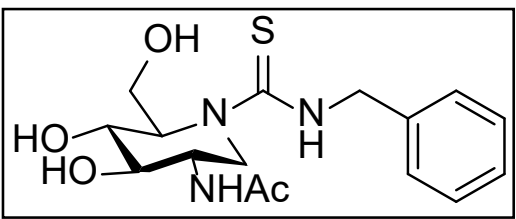

${ }^{1} \mathrm{H}$ NMR (500 MHz, $\left.\mathrm{CD}_{3} \mathrm{OD}\right): \delta$ 7.37-7.21 $\left(\mathrm{CH}_{\text {arom }}\right), 4.89$ (m, $\left.2 \mathrm{H}, \mathrm{H}-1 \mathrm{a}, \mathrm{CH}_{2} \mathrm{Ph}\right), 4.78\left(\mathrm{~d}, 1 \mathrm{H},{ }^{2} J_{\mathrm{H}, \mathrm{H}}=15 \mathrm{~Hz}, \mathrm{CH}_{2} \mathrm{Ph}\right), 4.38$ (m, $1 \mathrm{H}, \mathrm{H}-5), 3.95\left(\mathrm{dd}, 1 \mathrm{H}, J_{6 \mathrm{a}, 6 \mathrm{~b}}=11.4 \mathrm{~Hz}, J_{5,6 \mathrm{a}}=8.7 \mathrm{~Hz}\right.$, H-6a), 3.93 (m, 1 H, H-2), 3.83 (dd, 1 H, $J_{5,6 b}=3.6 \mathrm{~Hz}, \mathrm{H}-6 \mathrm{~b}$ ), 3.70 (t, $1 \mathrm{H}, J_{3,4}=J_{4,5}=5.1 \mathrm{~Hz}, \mathrm{H}-$ 4), $3.60\left(\mathrm{t}, 1 \mathrm{H}, J_{2,3}=5.1 \mathrm{~Hz}, \mathrm{H}-3\right), 3.46\left(\mathrm{dd}, 1 \mathrm{H}, J_{1 \mathrm{a}, 1 \mathrm{~b}}=14.3 \mathrm{~Hz}, J_{1 \mathrm{~b}, 2}=3.8 \mathrm{~Hz}, \mathrm{H}-1 \mathrm{~b}\right), 1.93$ (s, $\left.1 \mathrm{H}, \mathrm{COCH}_{3}\right) .{ }^{13} \mathrm{C}$ NMR $\left(125.7 \mathrm{MHz}, \mathrm{CD}_{3} \mathrm{OD}\right): \delta 186.9$ (CS), 173.0 (CO), 140.0, 129.4, 128.6, $128.0\left(\mathrm{C}_{\text {arom }}\right), 72.3$ (C-3), 70.6 (C-4), 66.0 (C-5), $61.8(\mathrm{C}-6), 53.0(\mathrm{C}-2), 50.5\left(\mathrm{CH}_{2} \mathrm{Ph}\right), 45.3$ (C1), $23.0\left(\mathrm{COCH}_{3}\right)$. ESIMS: $m / z 376[\mathrm{M}+\mathrm{Na}]^{+}$. Anal. Calcd for $\mathrm{C}_{16} \mathrm{H}_{23} \mathrm{~N}_{3} \mathrm{O}_{4} \mathrm{~S}: \mathrm{C}, 54.37 ; \mathrm{H}, 6.56$; N, 11.89; S, 9.07. Found: C, 54.45; H, 6.69; N, 11.76; S 8.84.

\section{2-Acetamido-5- $N$-(N'-1-naphthylmethylthiocarbamoyl)-1,2-dideoxynojirimycin}

(5).

Column chromatography, eluent 100:10:1 DCM-MeOH- $\mathrm{H}_{2} \mathrm{O}$. Yield: $90 \mathrm{mg}(60 \%)$. $[\alpha]_{\mathrm{D}}-54.6(c$

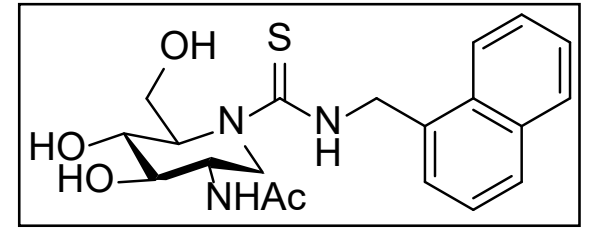
$1.00, \mathrm{MeOH}) . \mathrm{R}_{f} 0.40$ (70:10:1 DCM-MeOH- $\left.\mathrm{H}_{2} \mathrm{O}\right)$.

${ }^{1}$ H NMR (300 MHz, $\left.\mathrm{CD}_{3} \mathrm{OD}\right): \delta 8.10-7.41\left(\mathrm{CH}_{\text {arom }}\right), 5.27$ (bs, $\left.2 \mathrm{H}, \mathrm{CH}_{2} \mathrm{Naph}\right), 4.95$ (m, $\left.1 \mathrm{H}, \mathrm{H}-1 \mathrm{a}\right), 4.30$ (m, $1 \mathrm{H}$, 
H-5), $3.93\left(\mathrm{dd}, 1 \mathrm{H}, J_{6 \mathrm{a}, 6 \mathrm{~b}}=11.2 \mathrm{~Hz}, J_{5,6 \mathrm{a}}=8.8 \mathrm{~Hz}, \mathrm{H}-6 \mathrm{a}\right), 3.92(\mathrm{~m}, 1 \mathrm{H}, \mathrm{H}-2), 3.82\left(\mathrm{dd}, 1 \mathrm{H}, J_{5,6 \mathrm{~b}}\right.$ $=3.5 \mathrm{~Hz}, \mathrm{H}-6 \mathrm{~b}), 3.66$ (t, $\left.1 \mathrm{H}, J_{3,4}=J_{4,5}=5.7 \mathrm{~Hz}, \mathrm{H}-4\right), 3.61\left(\mathrm{t}, 1 \mathrm{H}, J_{2,3}=5.4 \mathrm{~Hz}, \mathrm{H}-3\right), 3.53$ (dd, $\left.1 \mathrm{H}, J_{1 \mathrm{a}, 1 \mathrm{~b}}=14.1 \mathrm{~Hz}, J_{1 \mathrm{~b}, 2}=3.8 \mathrm{~Hz}, \mathrm{H}-1 \mathrm{~b}\right), 1.94\left(\mathrm{~s}, 3 \mathrm{H}, \mathrm{NHCOCH}_{3}\right) .{ }^{13} \mathbf{C}$ NMR $(100.6 \mathrm{MHz}$, $\left.\mathrm{CD}_{3} \mathrm{OD}\right): \delta 185.3(\mathrm{CS}), 171.6(\mathrm{CO}), 133.9-123.2$ (C $\left.\mathrm{C}_{\text {arom }}\right), 70.9$ (C-3), 69.2 (C-4), 64.8 (C-5), 60.4 (C-6), $51.6(\mathrm{C}-2), 48.4\left(\mathrm{CH}_{2} \mathrm{Naph}\right), 43.9$ (C-1), $21.6\left(\mathrm{COCH}_{3}\right)$. ESIMS: $m / z 401.9$ [M - H] $]^{-}$. Anal. Calcd for $\mathrm{C}_{20} \mathrm{H}_{25} \mathrm{~N}_{3} \mathrm{O}_{4} \mathrm{~S}$ : C 59.53, H 6.25, N 10.41, S 7.95. Found: C, 59.42; H, 6.13; N, 10.29; S 7.80 .

2-Acetamido-5- $N$-( $N$ '-p-fluorobenzylthiocarbamoyl)-1,2-dideoxynojirimycin (6). Column chromatography, eluent 100:10:1 DCM-MeOH-H $\mathrm{H}_{2} \mathrm{O}$. Yield: $128 \mathrm{mg}(83 \%)$. [ $\left.\alpha\right]_{\mathrm{D}}-97.5$ (c 1.00, $\mathrm{MeOH}) . \mathrm{R}_{f} 0.33$ (70:10:1 DCM-MeOH-H$\left.{ }_{2} \mathrm{O}\right)$. UV (MeOH) $227 \mathrm{~nm}\left(\varepsilon_{\mathrm{mM}} 4.8\right)$.

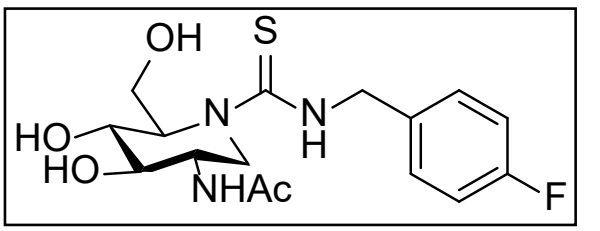

${ }^{1} \mathbf{H}$ NMR $\left(500 \mathrm{MHz}, \mathrm{CD}_{3} \mathrm{OD}\right): \delta 7.36,7.04\left(\mathrm{CH}_{\text {arom }}\right)$, $4.92(\mathrm{~m}, 1 \mathrm{H}, \mathrm{H}-1 \mathrm{a}), 4.76\left(\mathrm{~d}, 2 \mathrm{H},{ }^{3} J_{\mathrm{H}, \mathrm{H}}=15 \mathrm{~Hz}, \mathrm{CH}_{2} \mathrm{Ph}\right)$, $4.38(\mathrm{~m}, 1 \mathrm{H}, \mathrm{H}-5), 3.95\left(\mathrm{dd}, 1 \mathrm{H}, J_{6 \mathrm{a}, 6 \mathrm{~b}}=11.1 \mathrm{~Hz}, J_{5,6 \mathrm{a}}=\right.$

$8.9 \mathrm{~Hz}, \mathrm{H}-6 \mathrm{a}), 3.93$ (m, $1 \mathrm{H}, \mathrm{H}-2), 3.83$ (dd, $\left.1 \mathrm{H}, J_{5,6 \mathrm{~b}}=3.7 \mathrm{~Hz}, \mathrm{H}-6 \mathrm{~b}\right), 3.70$ (t, $1 \mathrm{H}, J_{3,4}=J_{4,5}=5.2$ $\mathrm{Hz}, \mathrm{H}-4), 3.60$ (t, $\left.1 \mathrm{H}, J_{2,3}=J_{3,4}=5.2 \mathrm{~Hz}, \mathrm{H}-3\right), 3.46$ (dd, $1 \mathrm{H}, J_{1 \mathrm{a}, 1 \mathrm{~b}}=14.3 \mathrm{~Hz}, J_{1 \mathrm{~b}, 2}=3.8 \mathrm{~Hz}, \mathrm{H}-$ 1b), 1.95 (s, $\left.3 \mathrm{H}, \mathrm{NHCOCH}_{3}\right) .{ }^{13} \mathbf{C}$ NMR (125.7 MHz, CD $\mathrm{OD}$ ): $\delta 185.4$ (CS), 171.6 (CO), 162.2, 134.8, 129.0, 114.6 (C $\mathrm{C}_{\text {arom}}$ ), 70.9 (C-3), 69.2 (C-4), 64.5 (C-5), 60.4 (C-6), 51.6 (C-2), 48.2 $\left(\mathrm{CH}_{2} \mathrm{Ph}\right), 43.9(\mathrm{C}-1), 21.6\left(\mathrm{COCH}_{3}\right)$. ESIMS: $m / z$ 394.1 $[\mathrm{M}+\mathrm{Na}]^{+}$. Anal. Calcd for $\mathrm{C}_{16} \mathrm{H}_{22} \mathrm{FN}_{3} \mathrm{O}_{4} \mathrm{~S}$ : C 51.74, H 5.97, N 11.31, S 8.63. Found: C, 51.57; H, 6.10; N, 11.19; S 8.36.

2-Acetamido-5- $N$-( $N$ '-p-methoxybenzylthiocarbamoyl)-1,2-dideoxynojirimycin (7). Column chromatography, eluent 100:10:1 DCM-MeOH-H ${ }_{2} \mathrm{O}$. Yield: $150 \mathrm{mg}(94 \%)$. [ $\left.\alpha\right]_{\mathrm{D}}-72.2$ (c 1.00, $\mathrm{MeOH}) . \mathrm{R}_{f} 0.40$ (70:10:1 DCM-MeOH-H $\left.2 \mathrm{O}\right)$. UV (MeOH) $230 \mathrm{~nm}\left(\varepsilon_{\mathrm{mm}} 31.4\right)$.

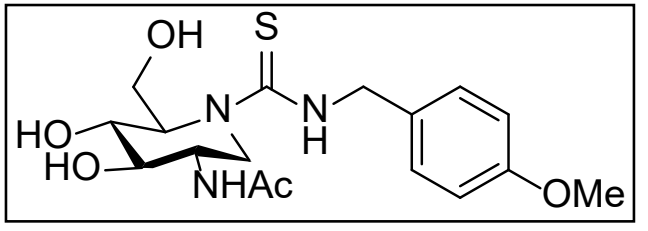

${ }^{1} \mathbf{H}$ NMR (500 MHz, $\left.\mathrm{CD}_{3} \mathrm{OD}\right): \delta 7.27(\mathrm{~m}, 2 \mathrm{H}$, $\left.\mathrm{CH}_{\text {arom }}\right), 6.8$ (m, $\left.2 \mathrm{H}, \mathrm{CH}_{\text {arom }}\right), 4.91$ (m, $\left.1 \mathrm{H}, \mathrm{H}-1 \mathrm{a}\right)$, $4.76\left(\mathrm{~d}, 2 \mathrm{H},{ }^{3} \mathrm{~J}_{H, H}=15 \mathrm{~Hz}, \mathrm{C} H_{2} \mathrm{Ph}\right), 4.34(\mathrm{~m}, 1 \mathrm{H}, \mathrm{H}-$ 5), $3.95\left(\mathrm{dd}, 1 \mathrm{H}, J_{6 \mathrm{a}, 6 \mathrm{~b}}=11.2 \mathrm{~Hz}, J_{5,6 \mathrm{a}}=8.7 \mathrm{~Hz}, \mathrm{H}-6 \mathrm{a}\right), 3.93(\mathrm{~m}, 1 \mathrm{H}, \mathrm{H}-2), 3.82\left(\mathrm{dd}, 1 \mathrm{H}, J_{5,6 \mathrm{~b}}=\right.$ $3.7 \mathrm{~Hz}, \mathrm{H}-6 \mathrm{~b}), 3.78$ (s, $3 \mathrm{H}, \mathrm{OCH}_{3}$ ), 3.69 (t, $\left.1 \mathrm{H}, J_{3,4}=J_{4,5}=5.4 \mathrm{~Hz}, \mathrm{H}-4\right), 3.60$ (t, $1 \mathrm{H}, J_{2,3}=J_{3,4}=$ $5.4 \mathrm{~Hz}, \mathrm{H}-3), 3.46\left(\mathrm{dd}, 1 \mathrm{H}, J_{1 \mathrm{a}, 1 \mathrm{~b}}=14.4 \mathrm{~Hz}, J_{1 \mathrm{~b}, 2}=3.7 \mathrm{~Hz}, \mathrm{H}-1 \mathrm{~b}\right), 1.95\left(\mathrm{~s}, 3 \mathrm{H}, \mathrm{NHCOCH}_{3}\right) .{ }^{13} \mathrm{C}$ NMR (125.7 MHz, CD $\left.{ }_{3} \mathrm{OD}\right): \delta 185.2(\mathrm{CS}), 171.6(\mathrm{CO}), 158.9,130.5,128.5,113.4\left(\mathrm{C}_{\text {arom }}\right), 71.0$ (C-3), 69.2 (C-4), 64.6 (C-5), $60.4(\mathrm{C}-6), 54.2\left(\mathrm{OCH}_{3}\right), 51.6(\mathrm{C}-2), 48.7\left(\mathrm{CH}_{2} \mathrm{Ph}\right), 43.9(\mathrm{C}-1), 21.6$ $\left(\mathrm{COCH}_{3}\right)$. ESIMS: $m / z 406.2[\mathrm{M}+\mathrm{Na}]^{+}$. Anal. Calcd for $\mathrm{C}_{17} \mathrm{H}_{25} \mathrm{~N}_{3} \mathrm{O}_{5} \mathrm{~S}: \mathrm{C} 53.25, \mathrm{H}$ 6.57, N 10.96, S 8.36. Found: C, 53.35; H, 6.72; N, 10.80; S 8.09. 
Column chromatography, eluent 100:10:1 DCM-MeOH-H $\mathrm{H}_{2} \mathrm{O}$. Yield: $87 \mathrm{mg}(55 \%)$. $[\alpha]_{\mathrm{D}}+4.2(c$ 1.00, MeOH). $\mathrm{R}_{f} 0.31$ (70:10:1 DCM-MeOH-H $\left.{ }_{2} \mathrm{O}\right)$. UV (MeOH) $223 \mathrm{~nm}\left(\varepsilon_{\mathrm{mm}} 15.4\right)$.

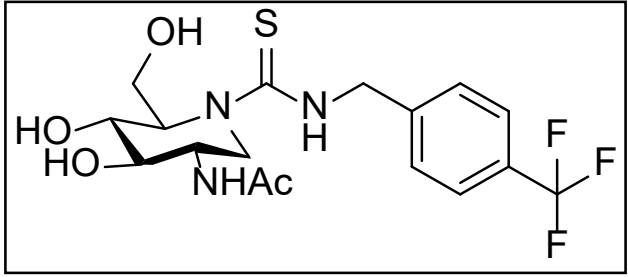

${ }^{1} \mathbf{H}$ NMR $\left(500 \mathrm{MHz}, \mathrm{CD}_{3} \mathrm{OD}\right): \delta 7.61\left(\mathrm{~d}, 2 \mathrm{H}, J_{\mathrm{H}, \mathrm{H}}=\right.$ $\left.7.9 \mathrm{~Hz}, \mathrm{CH}_{\text {arom }}\right), 7.52\left(\mathrm{~d}, 2 \mathrm{H}, \mathrm{CH}_{\text {arom }}\right), 5.01(\mathrm{~d}, 2 \mathrm{H}$, $\left.{ }^{3} J_{H, H}=15 \mathrm{~Hz}, \mathrm{CH}_{2} \mathrm{Ph}\right), 4.91(\mathrm{~m}, 1 \mathrm{H}, \mathrm{H}-1 \mathrm{a}), 4.47$ (m, $1 \mathrm{H}, \mathrm{H}-5), 4.12$ (m, $1 \mathrm{H}, \mathrm{H}-2), 3.99$ (dd, $1 \mathrm{H}, J_{6 \mathrm{a}, 6 \mathrm{~b}}=$ $\left.11.2 \mathrm{~Hz}, J_{5,6 \mathrm{a}}=8.7 \mathrm{~Hz}, \mathrm{H}-6 \mathrm{a}\right), 3.86\left(\mathrm{dd}, 1 \mathrm{H}, J_{5,6 \mathrm{~b}}=\right.$ $3.8 \mathrm{~Hz}, \mathrm{H}-6 \mathrm{~b}), 3.75\left(\mathrm{t}, 1 \mathrm{H}, J_{3,4}=J_{4,5}=5.2 \mathrm{~Hz}, \mathrm{H}-4\right), 3.63\left(\mathrm{dd}, 1 \mathrm{H}, J_{2,3}=7.2 \mathrm{~Hz}, J_{3,4}=5.2 \mathrm{~Hz}, \mathrm{H}-\right.$ 3), $3.46\left(\mathrm{dd}, 1 \mathrm{H}, J_{1 \mathrm{a}, 1 \mathrm{~b}}=14.1 \mathrm{~Hz}, J_{1 \mathrm{~b}, 2}=3.4 \mathrm{~Hz}, \mathrm{H}-1 \mathrm{~b}\right), 1.93(\mathrm{~s}, 3 \mathrm{H}, \mathrm{COCH})_{3} .{ }^{13} \mathbf{C} \mathbf{N M R}(125.7$ $\left.\mathrm{MHz}, \mathrm{CD}_{3} \mathrm{OD}\right): \delta 185.7(\mathrm{CS}), 171.6(\mathrm{CO}), 143.7,127.5\left(\mathrm{C}_{\text {arom }}\right), 124.7$ (q, $\left.\mathrm{CF}_{3}\right), 113.4\left(\mathrm{C}_{\text {arom }}\right), 70.7$ (C-3), 69.2 (C-4), 64.4 (C-5), 60.3 (C-6), $51.5(\mathrm{C}-2), 48.3\left(\mathrm{CH}_{2} \mathrm{Ph}\right), 43.9(\mathrm{C}-1), 21.6\left(\mathrm{COCH}_{3}\right)$. ESIMS: $m / z 444.1[\mathrm{M}+\mathrm{Na}]^{+}$. Anal. Calcd for $\mathrm{C}_{17} \mathrm{H}_{22} \mathrm{~F}_{3} \mathrm{~N}_{3} \mathrm{O}_{4} \mathrm{~S}$ : C 48.45, H 5.26, N 9.97, $\mathrm{S} 7.61$. Found: C, 48.36; H, 5.17; N, 9.72; S 7.36.

\section{2-Acetamido-5- $N$-(N'-o-azidomethylbenzylthiocarbamoyl)-1,2-dideoxynojirimycin}

(9).

Column chromatography, eluent 100:10:1 DCM-MeOH- $\mathrm{H}_{2} \mathrm{O}$. Yield: $114 \mathrm{mg}(75 \%)$. [ $\left.\alpha\right]_{\mathrm{D}}-44.0$ (c 1.00, MeOH). $\mathrm{R}_{f} 0.40$ (70:10:1 DCM-MeOH-H $\left.{ }_{2} \mathrm{O}\right)$. UV (MeOH) $230 \mathrm{~nm}\left(\varepsilon_{\mathrm{mm}} 20.3\right)$.

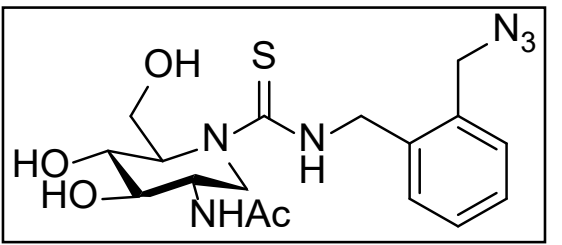

${ }^{1} \mathrm{H}$ NMR (300 MHz, $\left.\mathrm{CD}_{3} \mathrm{OD}\right): \delta$ 7.43-7.28 (d, $\left.4 \mathrm{H}, \mathrm{CH}_{\text {arom }}\right)$, 4.96 (bd, 1 H, H-1a), 4.91 (d, 2 H, $\mathrm{CH}_{2} \mathrm{Ph}$ ), 4.53 (s, $2 \mathrm{H}$, $\left.\mathrm{CH}_{2} \mathrm{~N}_{3}\right), 4.37(\mathrm{~m}, 1 \mathrm{H}, \mathrm{H}-5), 3.97\left(\mathrm{dd}, 1 \mathrm{H}, J_{6 \mathrm{a}, 6 \mathrm{~b}}=11.3 \mathrm{~Hz}\right.$, $\left.J_{5,6 \mathrm{a}}=8.5 \mathrm{~Hz}, \mathrm{H}-6 \mathrm{a}\right), 3.94(\mathrm{~m}, 1 \mathrm{H}, \mathrm{H}-2), 3.82\left(\mathrm{dd}, 1 \mathrm{H}, J_{5,6 \mathrm{~b}}\right.$ $=3.8 \mathrm{~Hz}, \mathrm{H}-6 \mathrm{~b}), 3.70$ (bdd, $\left.1 \mathrm{H}, J_{3,4}=J_{4,5}=5.4 \mathrm{~Hz}, \mathrm{H}-4\right), 3.61$ (t, $1 \mathrm{H}, J_{2,3}=5.4 \mathrm{~Hz}, \mathrm{H}-3$ ), 3.52 $\left(\mathrm{dd}, 1 \mathrm{H}, J_{1 \mathrm{a}, 1 \mathrm{~b}}=14.2 \mathrm{~Hz}, J_{1 \mathrm{~b}, 2}=3.7 \mathrm{~Hz}, \mathrm{H}-1 \mathrm{~b}\right), 1.95\left(\mathrm{~s}, 3 \mathrm{H}, \mathrm{COCH}_{3}\right) .{ }^{13} \mathbf{C}$ NMR $(100.6 \mathrm{MHz}$, $\left.\mathrm{CD}_{3} \mathrm{OD}\right): \delta 185.2(\mathrm{CS}), 171.6(\mathrm{CO}), 137.1,133.5,129.4,128.6,128.3,127.1$ (C $\left.\mathrm{C}_{\text {arom }}\right), 70.8$ (C-3), 69.2 (C-4), 64.5 (C-5), 60.4 (C-6), $51.9\left(\mathrm{CH}_{2} \mathrm{~N}_{3}\right), 51.5(\mathrm{C}-2), 46.4\left(\mathrm{CH}_{2} \mathrm{Ph}\right), 43.9(\mathrm{C}-1), 21.6$ $\left(\mathrm{COCH}_{3}\right)$. ESIMS: $m / z 431.2[\mathrm{M}+\mathrm{Na}]^{+}$. Anal. Calcd for $\mathrm{C}_{17} \mathrm{H}_{24} \mathrm{~N}_{6} \mathrm{O}_{4} \mathrm{~S}: \mathrm{C} 49.99, \mathrm{H}$ 5.92, N 20.57, S 7.85. Found: C, 49.81; H, 5.80; N, 20.36; S 7.64.

2-Acetamido-5- $N$-(N'-m-azidomethylbenzylthiocarbamoyl)-1,2-dideoxynojirimycin

(10). Column chromatography, eluent 100:10:1 DCM-MeOH-H $\mathrm{H}_{2} \mathrm{O}$. Yield: 94 mg (65\%). [ $\left.\alpha\right]_{\mathrm{D}}-64.3(c$ 1.00, MeOH). $\mathrm{R}_{f} 0.40$ (70:10:1 DCM-MeOH-H $\left.{ }_{2} \mathrm{O}\right)$. UV (MeOH) $230 \mathrm{~nm}\left(\varepsilon_{\mathrm{mm}} 31.4\right)$.

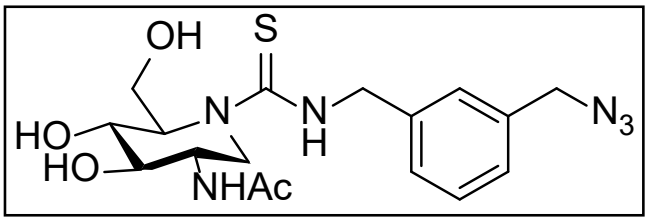

${ }^{1}$ H NMR (300 MHz, CD 30 OD): $\delta$ 7.27-7.11 (m, $4 \mathrm{H}$, $\mathrm{CH}_{\text {arom }}$ ), 4.83 (bd, $\left.1 \mathrm{H}, \mathrm{H}-1 \mathrm{a}\right), 4.78$ (bd, $2 \mathrm{H}, \mathrm{CH}_{2} \mathrm{Ph}$ ), $4.29(\mathrm{~m}, 1 \mathrm{H}, \mathrm{H}-5), 4.24\left(\mathrm{~s}, 2 \mathrm{H}, \mathrm{CH}_{2} \mathrm{~N}_{3}\right), 3.86(\mathrm{dd}, 1$ 
$\left.\mathrm{H}, J_{6 \mathrm{a}, 6 \mathrm{~b}}=11.3 \mathrm{~Hz}, J_{5,6 \mathrm{a}}=8.6 \mathrm{~Hz}, \mathrm{H}-6 \mathrm{a}\right), 3.83(\mathrm{~m}, 1 \mathrm{H}, \mathrm{H}-2), 3.72\left(\mathrm{dd}, 1 \mathrm{H}, J_{5,6 \mathrm{~b}}=3.8 \mathrm{~Hz}, \mathrm{H}-6 \mathrm{~b}\right)$, 3.61 (bdd, $\left.1 \mathrm{H}, J_{3,4}=J_{4,5}=5.3 \mathrm{~Hz}, \mathrm{H}-4\right), 3.50\left(\mathrm{t}, 1 \mathrm{H}, J_{2,3}=5.3 \mathrm{~Hz}, \mathrm{H}-3\right), 3.42\left(\mathrm{dd}, 1 \mathrm{H}, J_{1 \mathrm{a}, 1 \mathrm{~b}}=\right.$ $\left.14.2 \mathrm{~Hz}, J_{1 \mathrm{~b}, 2}=3.8 \mathrm{~Hz}, \mathrm{H}-1 \mathrm{~b}\right), 1.82\left(\mathrm{~s}, 3 \mathrm{H}, \mathrm{NHCOCH}_{3}\right) .{ }^{13} \mathbf{C} \mathbf{N M R}\left(100.6 \mathrm{MHz}, \mathrm{CD}_{3} \mathrm{OD}\right): \delta$ 185.5 (CS), 171.6 (CO), 139.5, 135.9, 128.4, 127.1, 126.9, 126.6 ( $\left.\mathrm{C}_{\text {arom }}\right), 70.8$ (C-3), 69.2 (C-4), 64.5 (C-5), $60.3(\mathrm{C}-6), 54.1\left(\mathrm{CH}_{2} \mathrm{~N}_{3}\right), 51.5(\mathrm{C}-2), 48.8\left(\mathrm{CH}_{2} \mathrm{Ph}\right), 43.8(\mathrm{C}-1), 21.6\left(\mathrm{COCH}_{3}\right)$. ESIMS: $m / z$ 431.2 [M + Na] $]^{+}$. Anal. Calcd for $\mathrm{C}_{17} \mathrm{H}_{24} \mathrm{~N}_{6} \mathrm{O}_{4} \mathrm{~S}$ : C 49.99, H 5.92, N 20.57, $\mathrm{S} 7.85$. Found: C, 49.75; H, 5.67; N, 20.20; S 7.51.

\section{2-Acetamido-5- $N$-(N'-p-azidomethylbenzylthiocarbamoyl)-1,2-dideoxynojirimycin}

(11).

Column chromatography, eluent 100:10:1 DCM-MeOH- $\mathrm{H}_{2} \mathrm{O}$. Yield: $100 \mathrm{mg}(60 \%)$. $[\alpha]_{\mathrm{D}}-54.0$ (c 1.00, MeOH). $\mathrm{R}_{f} 0.40$ (70:10:1 DCM-MeOH-H $\left.{ }_{2} \mathrm{O}\right)$. UV (MeOH) $230 \mathrm{~nm}\left(\varepsilon_{\mathrm{mm}} 31.4\right)$.

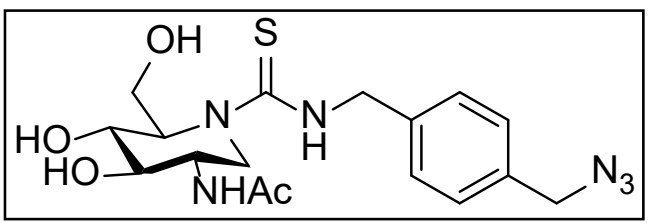

${ }^{1} \mathbf{H}$ NMR (400 MHz, $\left.\mathrm{CD}_{3} \mathrm{OD}\right): \delta 7.39\left(\mathrm{~d}, 2 \mathrm{H}, J_{\mathrm{H}, \mathrm{H}}=\right.$ $8.2 \mathrm{~Hz}, \mathrm{CH}_{\text {arom }}$ ), 7.32 (d, $\left.2 \mathrm{H}, \mathrm{CH}_{\text {arom }}\right), 4.94$ (bd, $1 \mathrm{H}$, $\mathrm{H}-1 \mathrm{a}), 4.82\left(\mathrm{~d}, 2 \mathrm{H},{ }^{2} J_{\mathrm{H}, \mathrm{H}}=15.0 \mathrm{~Hz}, \mathrm{CH}_{2} \mathrm{Ph}\right), 4.43$ (m, $1 \mathrm{H}, \mathrm{H}-5), 4.35\left(\mathrm{~s}, 2 \mathrm{H}, \mathrm{CH}_{2} \mathrm{~N}_{3}\right.$ ), $3.98\left(\mathrm{dd}, 1 \mathrm{H}, J_{6 \mathrm{a}, 6 \mathrm{~b}}=11.3 \mathrm{~Hz}, J_{5,6 \mathrm{a}}=8.5 \mathrm{~Hz}, \mathrm{H}-6 \mathrm{a}\right), 3.94$ (m, $1 \mathrm{H}, \mathrm{H}-2$ ), 3.85 (dd, $\left.1 \mathrm{H}, J_{5,6 \mathrm{~b}}=3.7 \mathrm{~Hz}, \mathrm{H}-6 \mathrm{~b}\right), 3.73$ (bdd, $\left.1 \mathrm{H}, J_{3,4}=J_{4,5}=5.4 \mathrm{~Hz}, \mathrm{H}-4\right), 3.62$ (t, 1 $\left.\mathrm{H}, J_{2,3}=5.4 \mathrm{~Hz}, \mathrm{H}-3\right), 3.54\left(\mathrm{dd}, 1 \mathrm{H}, J_{1 \mathrm{a}, 1 \mathrm{~b}}=14.2 \mathrm{~Hz}, J_{1 \mathrm{~b}, 2}=3.7 \mathrm{~Hz}, \mathrm{H}-1 \mathrm{~b}\right), 1.95(\mathrm{~s}, 3 \mathrm{H}$, $\left.\mathrm{NHCOCH}_{3}\right) .{ }^{13} \mathrm{C}$ NMR (100.6 MHz, CD $\left.{ }_{3} \mathrm{OD}\right): \delta 185.4$ (CS), 171.6 (CO), 139.0, 134.4, 128.1, 127.1 ( $\mathrm{C}_{\text {arom }}$ ), 70.8 (C-3), 69.2 (C-4), 64.5 (C-5), 60.4 (C-6), $53.9\left(\mathrm{CH}_{2} \mathrm{~N}_{3}\right), 51.6(\mathrm{C}-2), 48.7$ $\left(\mathrm{CH}_{2} \mathrm{Ph}\right), 43.9(\mathrm{C}-1), 21.6\left(\mathrm{COCH}_{3}\right)$. ESIMS: $m / z 431.2[\mathrm{M}+\mathrm{Na}]^{+}$. Anal. Calcd for $\mathrm{C}_{17} \mathrm{H}_{24} \mathrm{~N}_{6} \mathrm{O}_{4} \mathrm{~S}$ : C 49.99, H 5.92, N 20.57, S 7.85. Found: C, 49.75; H, 5.67; N, 20.20; S 7.51.

2-Acetamido-5- $N$-( $N$ '-2-phenylethylthiocarbamoyl)-1,2-dideoxynojirimycin (12). Column chromatography, eluent 100:10:1 DCM-MeOH-H $\mathrm{H}_{2} \mathrm{O}$. Yield: $120 \mathrm{mg}(78 \%)$. $[\alpha]_{\mathrm{D}}+4.29(c)$ 1.00, $\mathrm{MeOH}) . \mathrm{R}_{f} 0.42$ (70:10:1 DCM-MeOH-H $\left.2 \mathrm{O}\right)$. UV (MeOH) $223 \mathrm{~nm}\left(\varepsilon_{\mathrm{mm}} 15.4\right)$.

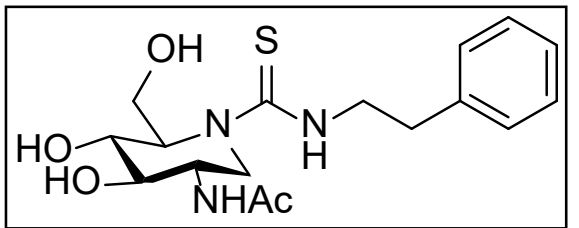

${ }^{1} \mathbf{H}$ NMR (300 MHz, CD $\left.30 D\right): \delta 7.33-7.17(\mathrm{~m}, 5 \mathrm{H}$, $\mathrm{CH}_{\text {arom }}$ ), 4.86 (bd, $\left.1 \mathrm{H}, \mathrm{H}-1 \mathrm{a}\right), 4.25$ (m, $\left.1 \mathrm{H}, \mathrm{H}-5\right), 3.92$ (m, $1 \mathrm{H}, \mathrm{H}-2), 3.91\left(\mathrm{dd}, 1 \mathrm{H}, J_{6 \mathrm{a}, 6 \mathrm{~b}}=11.2 \mathrm{~Hz}, J_{5,6 \mathrm{a}}=8.3 \mathrm{~Hz}\right.$, H-6a), 3.81 (m, 3 H, H-6b, $\mathrm{CH}_{2} \mathrm{CH}_{2} \mathrm{Ph}$ ), 3.69 (dd, 1 H, $J_{3,4}=5.9 \mathrm{~Hz}, J_{4,5}=4.6 \mathrm{~Hz}, \mathrm{H}-4$ ), 3.57 (bdd, $1 \mathrm{H}, \mathrm{H}-3), 3.48$ (dd, $\left.1 \mathrm{H}, J_{1 \mathrm{a}, 1 \mathrm{~b}}=14.2 \mathrm{~Hz}, J_{1 \mathrm{~b}, 2}=3.9 \mathrm{~Hz}, \mathrm{H}-1 \mathrm{~b}\right), 2.92\left(\mathrm{~m}, 2 \mathrm{H}, \mathrm{CH}_{2} \mathrm{CH}_{2} \mathrm{Ph}\right), 1.97$ (s, $\left.3 \mathrm{H}, \mathrm{NHCOCH}_{3}\right) .{ }^{13} \mathrm{C}$ NMR $\left(75.5 \mathrm{MHz}, \mathrm{CD}_{3} \mathrm{OD}\right): \delta 185.0$ (CS), 171.6 (CO), 139.2, 128.4, 128.0, 125.9 ( $\left.\mathrm{C}_{\text {arom }}\right), 71.1$ (C-3), 69.2 (C-4), 64.4 (C-5), 60.4 (C-6), $51.6(\mathrm{C}-2), 47.1\left(\mathrm{CH}_{2} \mathrm{CH}_{2} \mathrm{Ph}\right)$, $43.8(\mathrm{C}-1), 34.9\left(\mathrm{CH}_{2} \mathrm{CH}_{2} \mathrm{Ph}\right), 21.6\left(\mathrm{COCH}_{3}\right)$. ESIMS: $m / z 390.2[\mathrm{M}+\mathrm{Na}]^{+}$. Anal. Calcd for $\mathrm{C}_{17} \mathrm{H}_{25} \mathrm{~N}_{3} \mathrm{O}_{4} \mathrm{~S}$ : C 55.57, H 68.6, N 11.44, S 8.72. Found: C, 48.36; H, 5.17; N, 9.72; S 7.36. 


\section{Synthesis of DNJC-thiazolidines 13-24}

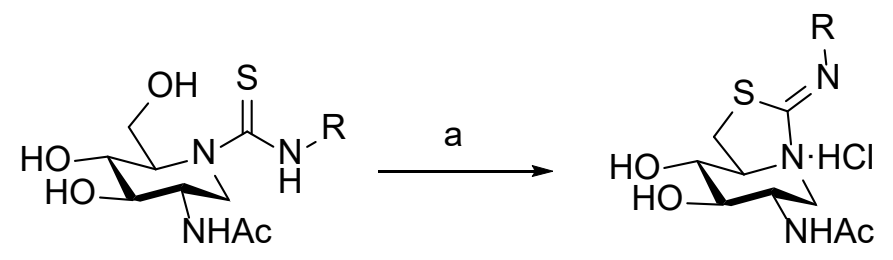

Supplemental scheme 5. Reagents and conditions: a. $\mathrm{HCl}_{(\text {conc), }}, \mathrm{MeOH}, \mathrm{RT}, 18 \mathrm{~h}$, quant.

General procedure for the preparation of the DNJNAc-thiazolidines 13-24. The corresponding DNJNAc thiourea 1-12 $(0.27 \mathrm{mM})$ was dissolved in $\mathrm{MeOH}(2 \mathrm{~mL})$ and concentrated $\mathrm{HCl}$ was dropwise added until $\mathrm{pH} 1$. The solution was stirred at RT until complete disappearance of the starting material $(18-24 \mathrm{~h})$. The solvent was removed under reduced pressure, the residue was coevaporated with $\mathrm{MeOH}(3 \times 10 \mathrm{~mL})$ and the product was purified by column chromatography using the eluent indicated in each case.

\section{(Z)-2-Acetamido-5- $N, 6-S$-( $N$ '-butyliminomethylidene)-1,2-dideoxy-6-thionojirimycin}

Hydrochloride (13). Column chromatography, eluent: 60:10:1 $\rightarrow$ 50:10:1 $\rightarrow$ 40:10:1 DCMMeOH-H ${ }_{2} \mathrm{O}$. Yield: $81 \mathrm{mg}$ (quantitative). $[\alpha]_{\mathrm{D}}+41.6$ (c 0.88, MeOH). $\mathrm{R}_{f}$ 0.25 (70:10:1 DCM$\left.\mathrm{MeOH}-\mathrm{H}_{2} \mathrm{O}\right)$.

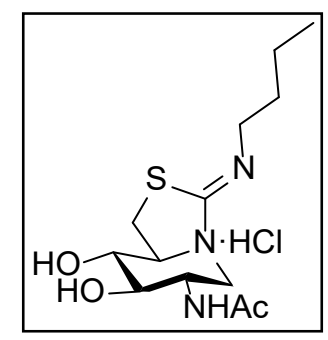

${ }^{1} \mathbf{H}$ NMR (500 MHz, CD $\left.\mathrm{CD}_{3} \mathrm{OD}\right): \delta 4.11\left(\mathrm{dd}, 1 \mathrm{H}, J_{1 \mathrm{a}, \mathrm{b}}=13.2 \mathrm{~Hz}, J_{1 \mathrm{a}, 2}=5.3\right.$ Hz, H-1a), 3.99 (m, 1 H, H-5), 3.83 (m, 1 H, H-2), 3.76 (dd, 1 H, $J_{6 \mathrm{a}, 6 \mathrm{~b}}=$ $\left.11.4 \mathrm{~Hz}, J_{5,6 \mathrm{a}}=7.7 \mathrm{~Hz}, \mathrm{H}-6 \mathrm{a}\right), 3.51$ (m, $\left.2 \mathrm{H}, \mathrm{H}-3, \mathrm{H}-6 \mathrm{~b}\right), 3.43$ (t, $1 \mathrm{H}, J_{3,4}$ $\left.=J_{4,5}=9.4, \mathrm{H}-4\right), 3.37\left(\mathrm{t}, 2 \mathrm{H}, \mathrm{CH}_{2} \mathrm{NH}\right), 3.01\left(\mathrm{dd}, 1 \mathrm{H}, J_{1 \mathrm{~b}, 2}=11.7 \mathrm{~Hz}, \mathrm{H}-\right.$ 1b), $2.01\left(\mathrm{~s}, 1 \mathrm{H}, \mathrm{COCH}_{3}\right), 1.66\left(\mathrm{~m}, 2 \mathrm{H}, \mathrm{CH}_{2}\right), 1.41\left(\mathrm{~m}, 2 \mathrm{H}, \mathrm{CH}_{2}\right), 0.97$ (t, $\left.3 \mathrm{H},{ }^{3} J_{\mathrm{H}, \mathrm{H}}=7.4 \mathrm{~Hz}, \mathrm{CH}_{3}\right) .{ }^{13} \mathbf{C}$ NMR $\left(125.7 \mathrm{MHz}, \mathrm{CD}_{3} \mathrm{OD}\right): \delta 173.9$ (CO), $171.8(\mathrm{CN}), 75.4$ (C-3), 74.7 (C-4), $69.1(\mathrm{C}-5), 50.9\left(\mathrm{CH}_{2} \mathrm{~N}\right), 50.4(\mathrm{C}-2), 46.9(\mathrm{C}-1), 32.2$ (C-6), $32.1\left(\mathrm{CH}_{2}\right), 22.7\left(\mathrm{COCH}_{3}\right), 20.8\left(\mathrm{CH}_{2}\right), 13.9\left(\mathrm{CH}_{3}\right)$. ESIMS: $m / z 302[\mathrm{M}+\mathrm{H}]^{+}, 324[\mathrm{M}+$ $\mathrm{Na}]^{+}$. Anal. Calcd for $\mathrm{C}_{13} \mathrm{H}_{23} \mathrm{~N}_{3} \mathrm{O}_{3}$ S.HCl: C, 46.21; H, 7.16; N, 12.44; S, 9.49. Found: C, 46.09; $\mathrm{H}, 7.22 ; \mathrm{N}, 12.15 ; \mathrm{S}, 9.13$. 
(Z)-2-Acetamido-1,2-dideoxy-5-N,6-S-(N'-octyliminomethylidene)-6-thionojirimycin

Hydrochloride (14). Column chromatography, eluent: 70:10:1 $\rightarrow$ 60:10:1 $\rightarrow$ 50:10:1 DCM$\mathrm{MeOH}-\mathrm{H}_{2} \mathrm{O}$. Yield: $96 \mathrm{mg}$ (quantitative). $[\alpha]_{\mathrm{D}}+36.3\left(c\right.$ 0.98, MeOH). $\mathrm{R}_{f}$ 0.53 (40:10:1 DCM$\mathrm{MeOH}-\mathrm{H}_{2} \mathrm{O}$ ).

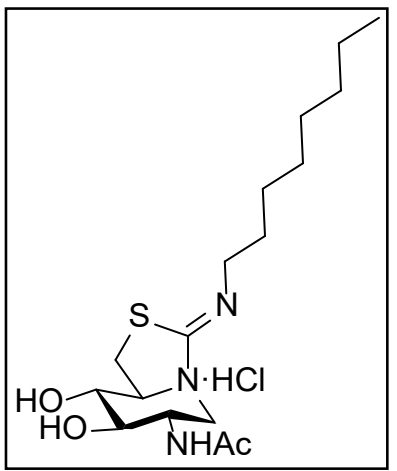

${ }^{1} \mathbf{H}$ NMR $\left(500 \mathrm{MHz}, \mathrm{CD}_{3} \mathrm{OD}\right): \delta 4.10\left(\mathrm{dd}, 1 \mathrm{H}, J_{1 \mathrm{a}, 1 \mathrm{~b}}=13.2 \mathrm{~Hz}, J_{1 \mathrm{a}, 2}=\right.$ $5.3 \mathrm{~Hz}, \mathrm{H}-1 \mathrm{a}), 3.96$ (m, $1 \mathrm{H}, \mathrm{H}-5), 3.82$ (m, $1 \mathrm{H}, \mathrm{H}-2), 3.73$ (dd, $1 \mathrm{H}$, $\left.J_{6 \mathrm{a}, 6 \mathrm{~b}}=11.4 \mathrm{~Hz}, J_{5,6 \mathrm{a}}=7.7 \mathrm{~Hz}, \mathrm{H}-6 \mathrm{a}\right), 3.49$ (m, $\left.2 \mathrm{H}, \mathrm{H}-6 \mathrm{~b}, \mathrm{H}-3\right), 3.42$ (t, $\left.1 \mathrm{H}, J_{3,4}=J_{4,5}=7.4 \mathrm{~Hz}, \mathrm{H}-4\right), 3.35\left(\mathrm{t}, 2 \mathrm{H}, \mathrm{CH}_{2} \mathrm{NH}\right), 2.98(\mathrm{t}, 1 \mathrm{H}$, $\left.J_{1 \mathrm{~b}, 2}=J_{1 \mathrm{a}, 1 \mathrm{~b}}=13.2 \mathrm{~Hz}, \mathrm{H}-1 \mathrm{~b}\right), 2.00\left(\mathrm{~s}, 1 \mathrm{H}, \mathrm{COCH}_{3}\right), 1.67(\mathrm{~m}, 2 \mathrm{H}$, $\left.\mathrm{CH}_{2}\right), 1.33\left(\mathrm{~m}, 10 \mathrm{H}, \mathrm{CH}_{2}\right), 0.91\left(\mathrm{t}, 3 \mathrm{H},{ }^{3} J_{\mathrm{H}, \mathrm{H}}=6.8 \mathrm{~Hz}, \mathrm{CH}_{3}\right) .{ }^{13} \mathbf{C}$ NMR (125.7 MHz, CD $\left.{ }_{3} \mathrm{OD}\right): \delta 173.9(\mathrm{CO}), 171.4(\mathrm{CN}), 75.5(\mathrm{C}-3)$, 74.8 (C-4), $67.0(\mathrm{C}-5), 50.9\left(\mathrm{CH}_{2} \mathrm{~N}\right), 50.9$ (C-2), 46.9 (C-1), 32.9 (C-6), 32.0, 30.3, 30.3, 30.2, 27.7, $23.7\left(\mathrm{CH}_{2}\right), 22.7\left(\mathrm{COCH}_{3}\right), 14.4\left(\mathrm{CH}_{3}\right)$. ESIMS: $m / z 358\left(100,[\mathrm{M}+\mathrm{H}]^{+}\right)$. Anal. Calcd for $\mathrm{C}_{17} \mathrm{H}_{31} \mathrm{~N}_{3} \mathrm{O}_{3} \mathrm{~S} . \mathrm{HCl}$ : C, 51.83; H, 8.19; N, 10.67; S, 8.14. Found: C, 52.00; H, 8.31; N, 10.44; S, 7.97.

(Z)-2-Acetamido-1,2-dideoxy-5-N,6-S-(N'-phenyliminomethylidene)-6-thionojirimycin Hydrochloride (15). Column chromatography, eluent: 60:10:1 $\rightarrow$ 50:10:1 $\rightarrow$ 40:10:1 DCMMeOH- $\mathrm{H}_{2} \mathrm{O}$. Yield: $86 \mathrm{mg}$ (quantitative). $[\alpha]_{\mathrm{D}}+12.2$ (c 0.89, MeOH). $\mathrm{R}_{f}$ 0.23 (70:10:1 DCM$\left.\mathrm{MeOH}-\mathrm{H}_{2} \mathrm{O}\right)$.

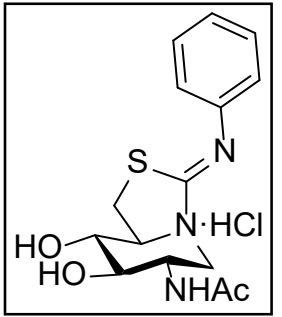

${ }^{1} \mathbf{H}$ NMR $\left(500 \mathrm{MHz}, 10: 1 \mathrm{CD}_{3} \mathrm{CN}-\mathrm{D}_{2} \mathrm{O}\right): \delta 7.32\left(\mathrm{t}, 2 \mathrm{H},{ }^{3} J_{\mathrm{H}, \mathrm{H}}=7.6 \mathrm{~Hz}, \mathrm{CH}-\right.$ arom), 7.14 (t, $\left.1 \mathrm{H}, \mathrm{CH}_{\text {arom }}\right), 7.0\left(\mathrm{~d}, 2 \mathrm{H}, \mathrm{CH}_{\text {arom }}\right), 4.21\left(\mathrm{dd}, 1 \mathrm{H}, J_{1 \mathrm{a}, 1 \mathrm{~b}}=13.1\right.$ $\left.\mathrm{Hz}, J_{1 \mathrm{a}, 2}=5.3 \mathrm{~Hz}, \mathrm{H}-1 \mathrm{a}\right), 3.81$ (m, $\left.1 \mathrm{H}, \mathrm{H}-2\right), 3.65$ (m, $\left.1 \mathrm{H}, \mathrm{H}-5\right), 3.47$ (t, $1 \mathrm{H}$, $\left.J_{2,3}=J_{3,4}=9.2 \mathrm{~Hz}, \mathrm{H}-3\right), 3.43\left(\mathrm{dd}, 1 \mathrm{H}, J_{6 \mathrm{a}, 6 \mathrm{~b}}=11.3 \mathrm{~Hz}, J_{5,6 \mathrm{a}}=7.2 \mathrm{~Hz}, \mathrm{H}-6 \mathrm{a}\right)$, $3.39\left(\mathrm{t}, 1 \mathrm{H}, J_{4,5}=9.2, \mathrm{H}-4\right), 3.18\left(\mathrm{dd}, 1 \mathrm{H}, J_{5,6 \mathrm{~b}}=6.9 \mathrm{~Hz}, \mathrm{H}-6 \mathrm{~b}\right), 2.79$ (dd, 1 $\left.\mathrm{H}, J_{1 \mathrm{~b}, 2}=11.5 \mathrm{~Hz}, \mathrm{H}-1 \mathrm{~b}\right), 1.94\left(\mathrm{~s}, 1 \mathrm{H}, \mathrm{COCH}_{3}\right) .{ }^{13} \mathbf{C ~ N M R}\left(125.7 \mathrm{MHz}, 10: 1 \mathrm{CD}_{3} \mathrm{CN}-\mathrm{D}_{2} \mathrm{O}\right): \delta$ $173.4(\mathrm{CO}), 164.1(\mathrm{CN}), 130.2-118.7\left(\mathrm{C}_{\text {arom }}\right), 75.8$ (C-3), 74.3 (C-4), 65.9 (C-5), 50.8 (C-2), 46.7 (C-1), $30.9(\mathrm{C}-6), 23.1\left(\mathrm{COCH}_{3}\right)$. ESIMS: $m / z 322[\mathrm{M}+\mathrm{H}]^{+}, 344[\mathrm{M}+\mathrm{Na}]^{+}$. Anal. Calcd for $\mathrm{C}_{15} \mathrm{H}_{19} \mathrm{~N}_{3} \mathrm{O}_{3} \mathrm{~S} . \mathrm{HCl}$ : C, 50.35; H, 5.63; N, 11.74; S, 8.96. Found: C, 50.67; H, 5.81; N, 11.42; S, 8.35 . 


\section{(Z)-2-Acetamido-5-N,6-S-( $N$ '-benzyliminomethylidene)-1,2-dideoxy-6-thionojirimycin}

Hydrochloride (16). Column chromatography, eluent: 60:10:1 DCM-MeOH- $\mathrm{H}_{2} \mathrm{O}$. Yield: $90 \mathrm{mg}$ (quantitative). $[\alpha]_{\mathrm{D}}+36.2\left(c\right.$ 1.04, MeOH). $\mathrm{R}_{f} 0.39\left(40: 10: 1 \mathrm{DCM}-\mathrm{MeOH}-\mathrm{H}_{2} \mathrm{O}\right)$.

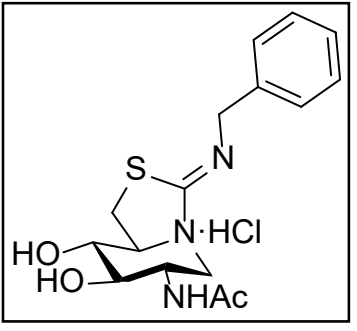

${ }^{1}$ H NMR (500 MHz, CD $\left.{ }_{3} \mathrm{OD}\right): \delta$ 7.37-7.27 (m, $\left.5 \mathrm{H}, \mathrm{CH}_{\text {arom }}\right), 4.49(\mathrm{~m}, 2$ $\left.\mathrm{H}, \mathrm{CH}_{2} \mathrm{Ph}\right), 4.16\left(\mathrm{dd}, 1 \mathrm{H}, J_{1 \mathrm{a}, 1 \mathrm{~b}}=13.1 \mathrm{~Hz}, J_{1 \mathrm{a}, 2}=5.3 \mathrm{~Hz}, \mathrm{H}-1 \mathrm{a}\right), 3.85(\mathrm{~m}$, $1 \mathrm{H}, \mathrm{H}-2), 3.77$ (m, $1 \mathrm{H}, \mathrm{H}-5), 3.63\left(\mathrm{dd}, 1 \mathrm{H}, J_{6 \mathrm{a}, 6 \mathrm{~b}}=11.2 \mathrm{~Hz}, J_{5,6 \mathrm{a}}=7.3\right.$ Hz, H-6a), 3.48 (t, $\left.1 \mathrm{H}, J_{2,3}=J_{3,4}=9.6, \mathrm{H}-3\right), 3.38$ (m, $1 \mathrm{H}, \mathrm{H}-4, \mathrm{H}-6 \mathrm{~b}$ ), $2.87\left(\mathrm{dd}, 1 \mathrm{H}, J_{1 \mathrm{~b}, 2}=11.7 \mathrm{~Hz}, \mathrm{H}-1 \mathrm{~b}\right), 1.99$ (s, $\left.1 \mathrm{H}, \mathrm{COCH}_{3}\right) .{ }^{13} \mathbf{C} \mathbf{~ N M R}$

(125.7 MHz, CD $\left.{ }_{3} \mathrm{OD}\right): \delta 173.8(\mathrm{CO}), 171.4(\mathrm{CN}), 138.5,129.7,128.8$ (C $\left.\mathrm{C}_{\text {arom }}\right), 76.0(\mathrm{C}-3), 74.0$ (C-4), 68.0 (C-5), 55.5 (CH $\left.\mathrm{CH}_{2} \mathrm{Ph}\right), 51.1(\mathrm{C}-2), 47.1$ (C-1), 31.9 (C-6), $22.7\left(\mathrm{COCH}_{3}\right)$. ESIMS: m/z $336[\mathrm{M}+\mathrm{H}]^{+}, 358[\mathrm{M}+\mathrm{Na}]^{+}$. Anal. Calcd for $\mathrm{C}_{16} \mathrm{H}_{22} \mathrm{~N}_{3} \mathrm{O}_{3}$ S.HCl: C, 51.68; H, 5.96; N, 11.30; $\mathrm{S}$, 8.62. Found: C, 51.82; H, 6.14; N, 11.09; S, 8.37.

\section{(Z)-2-Acetamido-5- $N, 6-S$-( $N$ '-1-naphthymethyliminomethylidene)-1,2-dideoxy-6-}

thionojirimycin Hydrochloride (17). Column chromatography, eluent: 70:10:1 DCM-MeOH$\mathrm{H}_{2} \mathrm{O}$. Yield: $104 \mathrm{mg}$ (quantitative). $[\alpha]_{\mathrm{D}}+4.8$ (c 1.00, 1:1 DCM-MeOH). $\mathrm{R}_{f} 0.62$ (70:10:1 DCM-

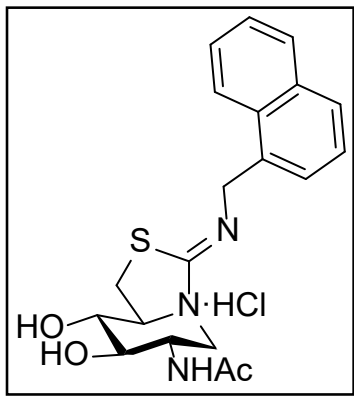
$\left.\mathrm{MeOH}-\mathrm{H}_{2} \mathrm{O}\right)$.

${ }^{1} \mathbf{H}$ NMR (300 MHz, 8:1 CD 3 OD-CDCl 3 ): $\delta$ 8.03-7.43 (m, $\left.2 \mathrm{H}, \mathrm{CH}_{\text {arom }}\right)$, 4.89 (bs, 2H, C $H_{2} \mathrm{Naph}$ ), $4.20\left(\mathrm{dd}, 1 \mathrm{H}, J_{1 \mathrm{a}, 1 \mathrm{~b}}=13.1 \mathrm{~Hz}, J_{1 \mathrm{a}, 2}=5.2 \mathrm{~Hz}\right.$, H-1a), 3.86 (m, 1 H, H-2), 3.65 (m, 1 H, H-5), 3.58 (m, 1 H, H-6a), 3.46 (t, $\left.1 \mathrm{H}, J_{2,3}=J_{3,4}=9.7, \mathrm{H}-3\right), 3.38(\mathrm{~m}, 2 \mathrm{H}, \mathrm{H}-4, \mathrm{H}-6 \mathrm{~b}), 2.78(\mathrm{dd}, 1 \mathrm{H}$, $\left.J_{1 \mathrm{~b}, 2}=11.5 \mathrm{~Hz}, \mathrm{H}-1 \mathrm{~b}\right), 1.98(\mathrm{~s}, 3 \mathrm{H}, \mathrm{NHCOCH}) .{ }^{13} \mathbf{C}$ NMR $(100.6 \mathrm{MHz}$, 8:1 $\left.\mathrm{CD}_{3} \mathrm{OD}-\mathrm{CDCl}_{3}\right): \delta 172.4(\mathrm{CO}), 165.5(\mathrm{CN}), 133.8-122.9\left(\mathrm{C}_{\text {arom }}\right), 75.0(\mathrm{C}-3), 73.7(\mathrm{C}-4), 65.9$ (C-5), 53.3 (CH $\left.\mathrm{CH}_{2} \mathrm{Naph}\right), 49.8$ (C-2), 45.8 (C-1), 30.4 (C-6), $21.5\left(\mathrm{NHCOCH}_{3}\right)$. ESIMS: $m / z$ 386.2 $[\mathrm{M}+\mathrm{H}]^{+}$. Anal. Calcd for $\mathrm{C}_{20} \mathrm{H}_{23} \mathrm{~N}_{3} \mathrm{O}_{3} \mathrm{~S} . \mathrm{HCl}$ : C 56.93, H 5.73, N 9.96, S 7.60. Found: C, 56.65; H, 5.48; N, 9.68; S 7.29.

\section{(Z)-2-Acetamido-5- $N, 6-S$-( $N$ '-p-fluorobenzyliminomethylidene)-1,2-dideoxy-6-}

thionojirimycin Hydrochloride (18). Column chromatography, eluent: 60:10:1 DCM-MeOH$\mathrm{H}_{2} \mathrm{O}$. Yield: $95 \mathrm{mg}$ (quantitative). $[\alpha]_{\mathrm{D}}+34.4$ (c 1.00, MeOH). $\mathrm{R}_{f} 0.53$ (40:10:1 DCM -MeOH-

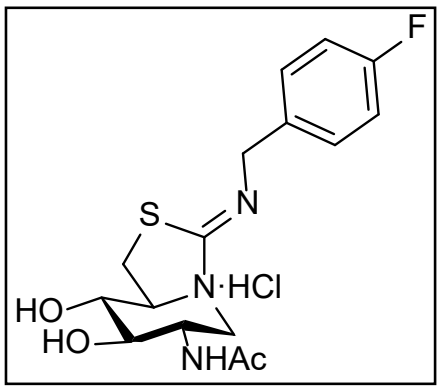
$\left.\mathrm{H}_{2} \mathrm{O}\right)$.

${ }^{1} \mathbf{H}$ NMR (500 MHz, $\left.\mathrm{CD}_{3} \mathrm{OD}\right): \delta 7.42\left(\mathrm{~m}, 2 \mathrm{H}, \mathrm{CH}_{\text {arom }}\right), 7.12$ (bt, 2 $\left.\mathrm{H}, J_{\mathrm{H}, \mathrm{H}}=8.7 \mathrm{~Hz}, \mathrm{CH}_{\text {arom }}\right), 4.54$ (bs, $\left.2 \mathrm{H}, \mathrm{CH}_{2} \mathrm{Ph}\right), 4.17$ (dd, $1 \mathrm{H}, J_{1 \mathrm{a}, 1 \mathrm{~b}}$ $\left.=13.1 \mathrm{~Hz}, J_{1 \mathrm{a}, 2}=5.2 \mathrm{~Hz}, \mathrm{H}-1 \mathrm{a}\right), 3.94(\mathrm{~m}, 1 \mathrm{H}, \mathrm{H}-2), 3.86(\mathrm{~m}, 1 \mathrm{H}$, H-5), $3.72\left(\mathrm{dd}, 1 \mathrm{H}, J_{6 \mathrm{a}, 6 \mathrm{~b}}=11.1 \mathrm{~Hz}, J_{5,6 \mathrm{a}}=7.5 \mathrm{~Hz}, \mathrm{H}-6 \mathrm{a}\right), 3.53(\mathrm{t}$, 
$\left.1 \mathrm{H}, J_{2,3}=J_{3,4}=9.4 \mathrm{~Hz}, \mathrm{H}-3\right), 3.40$ (m, $\left.2 \mathrm{H}, \mathrm{H}-4, \mathrm{H}-6 \mathrm{~b}\right), 3.00$ (dd, $\left.1 \mathrm{H}, J_{1 \mathrm{~b}, 2}=11.7 \mathrm{~Hz}, \mathrm{H}-1 \mathrm{~b}\right)$, 2.02 (s, $\left.1 \mathrm{H}, \mathrm{NHCOCH}_{3}\right) .{ }^{13} \mathrm{C}$ NMR $\left(125.7 \mathrm{MHz}, \mathrm{CD}_{3} \mathrm{OD}\right): \delta 172.3(\mathrm{CO}), 169.5(\mathrm{CN}), 162,128.6$, 113.5 ( $\mathrm{C}_{\text {arom}}$ ), 74.2 (C-3), 73.4 (C-4), 67.2 (C-5), $52.1\left(\mathrm{CH}_{2} \mathrm{Ph}\right), 49.5(\mathrm{C}-2), 45.6(\mathrm{C}-1), 30.7$ (C6), $21.3\left(\mathrm{NHCOCH}_{3}\right)$. ESIMS: $m / z 354[\mathrm{M}+\mathrm{H}]^{+}, 376[\mathrm{M}+\mathrm{Na}]^{+}$. Anal. Calcd for $\mathrm{C}_{16} \mathrm{H}_{20} \mathrm{FN}_{3} \mathrm{O}_{3} \mathrm{~S} . \mathrm{HCl}$ : C 49.29, H 5.43, N 10.78, S 8.22. Found: C, 49.14; H, 5.53; N, 10.62; S, 7.99 .

(Z)-2-Acetamido-5-N,6-S-( $N$ '-p-methoxybenzyliminomethylidene)-1,2-dideoxy-6-

thionojirimycin Hydrochloride (19). Column chromatography, eluent: 60:10:1 DCM-MeOH$\mathrm{H}_{2} \mathrm{O}$. Yield: $98 \mathrm{mg}$ (quantitative). $[\alpha]_{\mathrm{D}}+36.2$ (c 1.00, MeOH). $\mathrm{R}_{f} 0.32$ (40:10:1 DCM-MeOH$\mathrm{H}_{2} \mathrm{O}$ ).

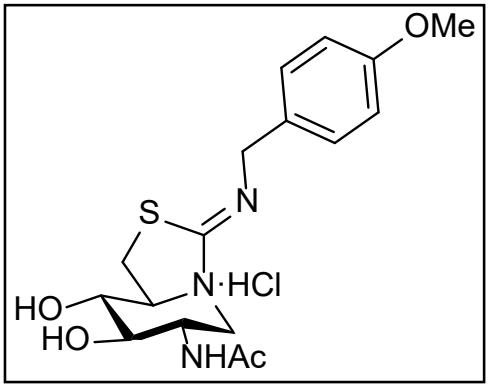

${ }^{1} \mathbf{H}$ NMR $\left(500 \mathrm{MHz}, \mathrm{CD}_{3} \mathrm{OD}\right): \delta 7.26\left(\mathrm{~d}, 2 \mathrm{H}, J_{\mathrm{H}, \mathrm{H}}=8.5 \mathrm{~Hz}\right.$, $\left.\mathrm{CH}_{\text {arom }}\right), 6.91\left(\mathrm{~d}, 2 \mathrm{H}, J_{\mathrm{H}, \mathrm{H}}=8.5 \mathrm{~Hz}, \mathrm{Ph}\right), 4.41\left(\mathrm{~d}, 2 \mathrm{H},{ }^{3} J_{\mathrm{H}, \mathrm{H}}=15\right.$ $\left.\mathrm{Hz}, \mathrm{CH}_{2} \mathrm{Ph}\right), 4.15\left(\mathrm{dd}, 1 \mathrm{H}, J_{1 \mathrm{a}, 1 \mathrm{~b}}=13.1 \mathrm{~Hz}, J_{1 \mathrm{a}, 2}=5.4 \mathrm{~Hz}, \mathrm{H}-\right.$ 1a), 3.84 (m, $1 \mathrm{H}, \mathrm{H}-2), 3.79$ (s, $\left.3 \mathrm{H}, \mathrm{OCH}_{3}\right), 3.69$ (m, $1 \mathrm{H}, \mathrm{H}-$ 5), $3.63\left(\mathrm{dd}, 1 \mathrm{H}, J_{6 \mathrm{a}, 6 \mathrm{~b}}=11.1 \mathrm{~Hz}, J_{5,6 \mathrm{a}}=7.2 \mathrm{~Hz}, \mathrm{H}-6 \mathrm{a}\right), 3.46$ (t, $\left.1 \mathrm{H}, J_{2,3}=J_{3,4}=9.7, \mathrm{H}-3\right), 3.38$ (m, $\left.2 \mathrm{H}, \mathrm{H}-4, \mathrm{H}-6 \mathrm{~b}\right), 2.87$ (dd, 1

$\left.\mathrm{H}, J_{1 \mathrm{~b}, 2}=11.7 \mathrm{~Hz}, \mathrm{H}-1 \mathrm{~b}\right), 2.00$ (s, $3 \mathrm{H}, \mathrm{NHCOCH}_{3}$ ). ${ }^{13} \mathbf{C}$ NMR (125.7 MHz, CD $\mathrm{OD}$ ): $\delta 172.3$ (CO), 168.1 (CN), 159.2, 128.6, $113.5\left(\mathrm{C}_{\text {arom }}\right), 74.8(\mathrm{C}-3), 73.6(\mathrm{C}-4), 66.2(\mathrm{C}-5), 54.3\left(\mathrm{CH}_{2} \mathrm{Ph}\right.$, $\left.\mathrm{CH}_{3} \mathrm{O}\right), 49.7$ (C-2), 45.7 (C-1), $30.3(\mathrm{C}-6), 21.3\left(\mathrm{NHCOCH}_{3}\right)$. ESIMS: $m / z 366.2[\mathrm{M}+\mathrm{H}]^{+}, 388.1$ $[\mathrm{M}+\mathrm{Na}]^{+}$. Anal. Calcd for $\mathrm{C}_{17} \mathrm{H}_{23} \mathrm{~N}_{3} \mathrm{O}_{4} \mathrm{~S} . \mathrm{HCl}: \mathrm{C} 50.80, \mathrm{H}$ 6.02, N 10.46, S 7.98. Found: C, 50.53; $\mathrm{H}, 6.14 ; \mathrm{N}, 10.33 ; \mathrm{S}, 7.75$.

\section{(Z)-2-Acetamido-5-N,6-S-( $N$ '-p-trifluoromethylbenzyliminomethylidene)-1,2-dideoxy-6-}

thionojirimycin Hydrochloride (20). Column chromatography, eluent: 60:10:1 DCM-MeOH$\mathrm{H}_{2} \mathrm{O}$. Yield: $95 \mathrm{mg}$ (quantitative). $[\alpha]_{\mathrm{D}}+34.4$ (c 1.00, MeOH). $\mathrm{R}_{f} 0.50$ (40:10:1 DCM-MeOH$\mathrm{H}_{2} \mathrm{O}$ ).

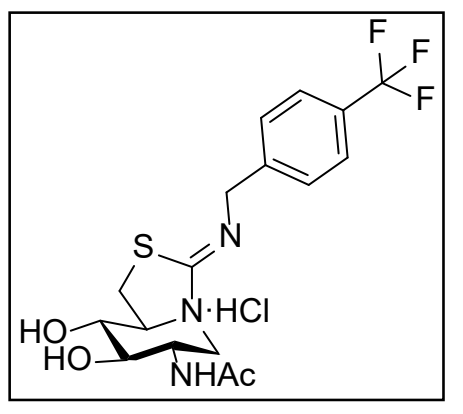

${ }^{1} \mathbf{H}$ NMR (500 MHz, $\left.\mathrm{CD}_{3} \mathrm{OD}\right): \delta 7.42\left(\mathrm{~m}, 2 \mathrm{H}, \mathrm{CH}_{\text {arom }}\right), 7.12$ (bt, 2 $\left.\mathrm{H}, J_{\mathrm{H}, \mathrm{H}}=8.7 \mathrm{~Hz}, \mathrm{CH}_{\text {arom}}\right), 4.54\left(\mathrm{bs}, 2 \mathrm{H}, \mathrm{CH}_{2} \mathrm{Ph}\right), 4.17(\mathrm{dd}, 1 \mathrm{H}$, $\left.J_{1 \mathrm{a}, 1 \mathrm{~b}}=13.1 \mathrm{~Hz}, J_{1 \mathrm{a}, 2}=5.2 \mathrm{~Hz}, \mathrm{H}-1 \mathrm{a}\right), 3.94(\mathrm{~m}, 1 \mathrm{H}, \mathrm{H}-2), 3.86$ (m, $1 \mathrm{H}, \mathrm{H}-5), 3.72\left(\mathrm{dd}, 1 \mathrm{H}, J_{6 \mathrm{a}, 6 \mathrm{~b}}=11.1 \mathrm{~Hz}, J_{5,6 \mathrm{a}}=7.5 \mathrm{~Hz}, \mathrm{H}-6 \mathrm{a}\right), 3.53$ (t, $\left.1 \mathrm{H}, J_{2,3}=J_{3,4}=9.4, \mathrm{H}-3\right), 3.40$ (m, $\left.2 \mathrm{H}, \mathrm{H}-4, \mathrm{H}-6 \mathrm{~b}\right), 3.00$ (dd, $\left.1 \mathrm{H}, J_{1 \mathrm{~b}, 2}=11.7 \mathrm{~Hz}, \mathrm{H}-1 \mathrm{~b}\right), 2.02\left(\mathrm{~s}, 3 \mathrm{H}, \mathrm{NHCOCH}_{3}\right) .{ }^{13} \mathbf{C} \mathbf{N M R}$ (125.7 MHz, $\left.\mathrm{CD}_{3} \mathrm{OD}\right): \delta 172.34(\mathrm{CO}), 163.7(\mathrm{CN}), 128.6,113.5$ (Carom), 74.2 (C-3), 73.4 (C-4), 67.2 (C-5), $52.1\left(\mathrm{CH}_{2} \mathrm{Ph}\right), 49.5$ (C-2), 45.6 (C-1), 30.7 (C-6), $21.3\left(\mathrm{NHCOCH}_{3}\right)$. ESIMS: m/z 
354.1 $[\mathrm{M}+\mathrm{H}]^{+}, 376.1[\mathrm{M}+\mathrm{Na}]^{+}$. Anal. Calcd for $\mathrm{C}_{16} \mathrm{H}_{20} \mathrm{FN}_{3} \mathrm{O}_{3} \mathrm{~S} . \mathrm{HCl}$ : C 49.29, H 5.43, N 10.78, S 8.22. Found: C, 49.14; H, 5.53; N, 10.62; S, 7.99.

(Z)-2-Acetamido-5- $N, 6-S$-( $N$ '-o-azidomethylbenzyliminomethylidene)-1,2-dideoxy-6-

thionojirimycin Hydrochloride (21). Column chromatography, eluent: 70:10:1 DCM-MeOH$\mathrm{H}_{2} \mathrm{O}$. Yield: $105 \mathrm{mg}$ (quantitative). $[\alpha]_{\mathrm{D}}+26.4$ (c 1.00, MeOH). $\mathrm{R}_{f} 0.75$ (50:10:1 DCM-MeOH$\mathrm{H}_{2} \mathrm{O}$ ).

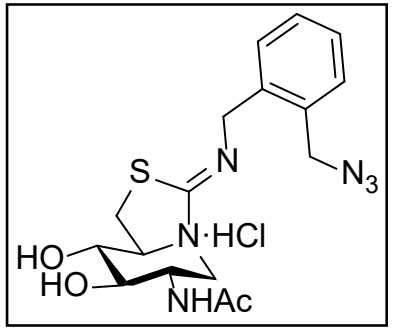

${ }^{1} \mathbf{H}$ NMR (300 MHz, $\left.\mathrm{CD}_{3} \mathrm{OD}\right): \delta$ 7.31-7.13 (m, $\left.4 \mathrm{H}, \mathrm{CH}_{\text {arom }}\right), 4.36$ (s, $\left.2 \mathrm{H}, \mathrm{NCH}_{2} \mathrm{Ph}\right), 4.33\left(\mathrm{bd}, 2 \mathrm{H}, \mathrm{CH}_{2} \mathrm{~N}_{3}\right), 4.07\left(\mathrm{dd}, 1 \mathrm{H}, J_{1 \mathrm{a}, 1 \mathrm{~b}}=12.9 \mathrm{~Hz}\right.$, $\left.J_{1 \mathrm{a}, 2}=5.2 \mathrm{~Hz}, \mathrm{H}-1 \mathrm{a}\right), 3.73(\mathrm{~m}, 1 \mathrm{H}, \mathrm{H}-2), 3.37$ (bdd, $1 \mathrm{H}, J_{6 \mathrm{a}, 6 \mathrm{~b}}=11.1$ $\left.\mathrm{Hz}, J_{5,6 \mathrm{a}}=6.2 \mathrm{~Hz}, \mathrm{H}-6 \mathrm{a}\right), 3.33$ (m, $1 \mathrm{H}, \mathrm{H}-5$ ), 3.26 (m, $2 \mathrm{H}, \mathrm{H}-3, \mathrm{H}-4$ ), $3.10(\mathrm{dd}, 1 \mathrm{H}, \mathrm{H}-6 \mathrm{~b}), 2.50$ (dd, $\left.1 \mathrm{H}, J_{1 \mathrm{~b}, 2}=11.3 \mathrm{~Hz}, \mathrm{H}-1 \mathrm{~b}\right), 1.86$ (s, 3

$\left.\mathrm{H}, \mathrm{NHCOCH}_{3}\right) .{ }^{13} \mathbf{C}$ NMR $\left(75.5 \mathrm{MHz}, \mathrm{CD}_{3} \mathrm{OD}\right): \delta 172.2(\mathrm{CO}), 162.9(\mathrm{CN}), 138.5,133.4,129.3$, 128.5, 128.2, 126.8 (C $\left.\mathrm{C}_{\text {arom }}\right), 75.5(\mathrm{C}-3), 73.8(\mathrm{C}-4), 64.7(\mathrm{C}-5), 54.8\left(\mathrm{CH}_{2} \mathrm{Ph}\right), 51.9\left(\mathrm{CH}_{2} \mathrm{~N}_{3}\right), 49.9$ (C-2), 45.7 (C-1), 29.9 (C-6), $21.3\left(\mathrm{NHCOCH}_{3}\right)$. ESIMS: m/z $391.1[\mathrm{M}+\mathrm{H}]^{+}$. Anal. Calcd for $\mathrm{C}_{17} \mathrm{H}_{22} \mathrm{~N}_{6} \mathrm{O}_{3} \mathrm{~S} . \mathrm{HCl}$ : C 47.83, H 5.43, N 19.69, S 7.51. Found: C, 47.51; H, 5.19; N, 19.33; S, 7.14.

(Z)-2-Acetamido-5-N,6-S-(N'-m-azidomethylbenzyliminomethylidene)-1,2-dideoxy-6-

thionojirimycin Hydrochloride (22). Column chromatography, eluent: 70:10:1 DCM-MeOH$\mathrm{H}_{2} \mathrm{O}$. Yield: $105 \mathrm{mg}$ (quantitative). $[\alpha]_{\mathrm{D}}+37.4$ (c 1.00, MeOH). $\mathrm{R}_{f} 0.75$ (50:10:1 DCM-MeOH$\mathrm{H}_{2} \mathrm{O}$ ).

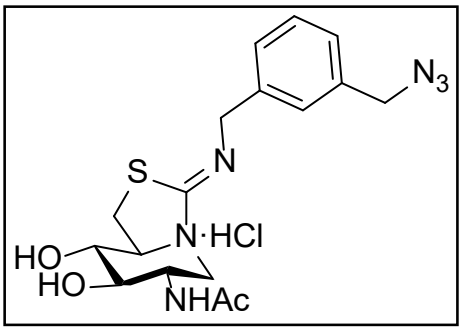

${ }^{1}$ H NMR (300 MHz, $\left.\mathrm{CD}_{3} \mathrm{OD}\right): \delta$ 7.38-7.22 (m, $\left.4 \mathrm{H}, \mathrm{CH}_{\text {arom }}\right), 4.42$ (bd, $\left.2 \mathrm{H}, \mathrm{NCH}_{2} \mathrm{Ph}\right), 4.36\left(\mathrm{~s}, 2 \mathrm{H}, \mathrm{CH}_{2} \mathrm{~N}_{3}\right), 4.18\left(\mathrm{dd}, 1 \mathrm{H}, J_{1 \mathrm{a}, 1 \mathrm{~b}}=\right.$ $\left.12.9 \mathrm{~Hz}, J_{1 \mathrm{a}, 2}=5.2 \mathrm{~Hz}, \mathrm{H}-1 \mathrm{a}\right), 3.87$ (m, $\left.1 \mathrm{H}, \mathrm{H}-2\right), 3.50$ (bdd, $1 \mathrm{H}$, $\left.J_{6 \mathrm{a}, 6 \mathrm{~b}}=11.1 \mathrm{~Hz}, J_{5,6 \mathrm{a}}=J_{5,6 \mathrm{~b}}=6.2 \mathrm{~Hz}, \mathrm{H}-6 \mathrm{a}\right), 3.45(\mathrm{~m}, 1 \mathrm{H}, \mathrm{H}-5)$, 3.39 (m, 2 H, H-3, H-4), 3.22 (dd, 1 H, H-6b), 2.65 (dd, 1 H, J J ,2 $=11.3 \mathrm{~Hz}, \mathrm{H}-1 \mathrm{~b}), 1.99$ (s, $\left.3 \mathrm{H}, \mathrm{NHCOCH}_{3}\right) .{ }^{13} \mathbf{C}$ NMR (75.5 MHz, CD ${ }_{3} \mathrm{OD}$ ): $\delta 172.2(\mathrm{CO}), 162.9$ $(\mathrm{CN}), 140.4,135.8,128.4,127.0,126.4\left(\mathrm{C}_{\text {arom }}\right), 75.5$ (C-3), $73.9(\mathrm{C}-4), 64.9(\mathrm{C}-5), 56.8\left(\mathrm{CH}_{2} \mathrm{Ph}\right)$, $54.1\left(\mathrm{CH}_{2} \mathrm{~N}_{3}\right), 49.9(\mathrm{C}-2), 45.8$ (C-1), $30.0(\mathrm{C}-6), 21.3\left(\mathrm{NHCOCH}_{3}\right)$. ESIMS: $m / z 391.1[\mathrm{M}+$ $\mathrm{H}]^{+}$. Anal. Calcd for $\mathrm{C}_{17} \mathrm{H}_{22} \mathrm{~N}_{6} \mathrm{O}_{3} \mathrm{~S} . \mathrm{HCl}$ : C 47.83, H 5.43, N 19.69, S 7.51. Found: C, 47.69; H, 5.24; N, 19.39; S, 7.20. 
(Z)-2-Acetamido-5-N,6-S-(N'-p-azidomethylbenzyliminomethylidene)-1,2-dideoxy-6-

thionojirimycin Hydrochloride (23). Column chromatography, eluent: 70:10:1 DCM-MeOH$\mathrm{H}_{2} \mathrm{O}$. Yield: $105 \mathrm{mg}$ (quantitative). $[\alpha]_{\mathrm{D}}+1.07$ (c 1.00, MeOH). $\mathrm{R}_{f} 0.75$ (50:10:1 DCM-MeOH$\left.\mathrm{H}_{2} \mathrm{O}\right)$.

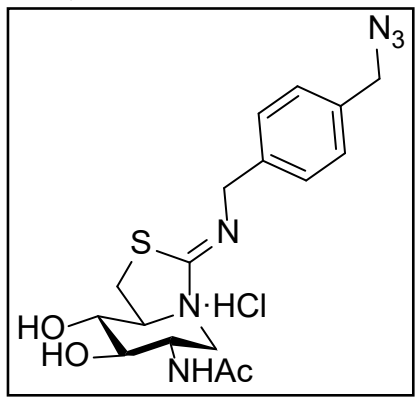

${ }^{1} \mathbf{H}$ NMR (400 MHz, CD $\left.{ }_{3} \mathrm{OD}\right): \delta 7.35\left(\mathrm{~d}, 2 \mathrm{H}, J_{\mathrm{H}, \mathrm{H}}=8.3 \mathrm{~Hz}\right.$, $\left.\mathrm{CH}_{\text {arom }}\right), 7.30\left(\mathrm{~d}, 2 \mathrm{H}, \mathrm{CH}_{\text {arom}}\right), 4.40$ (bt, $2 \mathrm{H},{ }^{2} J_{\mathrm{H}, \mathrm{H}}=15.0 \mathrm{~Hz}$, $\left.\mathrm{NCH}_{2} \mathrm{Ph}\right), 4.34\left(\mathrm{~s}, 2 \mathrm{H}, \mathrm{CH}_{2} \mathrm{~N}_{3}\right), 4.18\left(\mathrm{dd}, 1 \mathrm{H}, J_{1 \mathrm{a}, 1 \mathrm{~b}}=12.9 \mathrm{~Hz}, J_{1 \mathrm{a}, 2}\right.$ $=5.2 \mathrm{~Hz}, \mathrm{H}-1 \mathrm{a}), 3.88$ (m, $1 \mathrm{H}, \mathrm{H}-2), 3.47$ (m, $1 \mathrm{H}, \mathrm{H}-5), 3.39$ (m, 2 H, H-3, H-4), 3.34 (m, 1 H, H-6a), 3.19 (dd, 1 H, $J_{6 a, 6 b}=11.1 \mathrm{~Hz}$, $\left.J_{5,6 \mathrm{~b}}=6.8 \mathrm{~Hz}, \mathrm{H}-6 \mathrm{~b}\right), 2.61\left(\mathrm{dd}, 1 \mathrm{H}, J_{1 \mathrm{~b}, 2}=11.3 \mathrm{~Hz}, \mathrm{H}-1 \mathrm{~b}\right), 2.00$ (s,

$\left.3 \mathrm{H}, \mathrm{NHCOCH}_{3}\right) .{ }^{13} \mathrm{C}$ NMR (100.6 MHz, $\left.\mathrm{CD}_{3} \mathrm{OD}\right): \delta 172.2(\mathrm{CO}), 161.9(\mathrm{CN}), 140.4,134.0$, 128.0, $127.5\left(\mathrm{C}_{\text {arom}}\right), 75.6(\mathrm{C}-3), 74.0(\mathrm{C}-4), 64.6(\mathrm{C}-5), 54.2\left(\mathrm{CH}_{2} \mathrm{Ph}\right), 53.9\left(\mathrm{CH}_{2} \mathrm{~N}_{3}\right), 49.9(\mathrm{C}-2)$, $45.8(\mathrm{C}-1), 29.8(\mathrm{C}-6), 21.3\left(\mathrm{NHCOCH}_{3}\right)$. ESIMS: $m / z 391.1[\mathrm{M}+\mathrm{H}]^{+}$. Anal. Calcd for $\mathrm{C}_{17} \mathrm{H}_{22} \mathrm{~N}_{6} \mathrm{O}_{3} \mathrm{~S} . \mathrm{HCl}$ : C 47.83, H 5.43, N 19.69, S 7.51. Found: C, 47.49; H, 5.19; N, 19.43; S, 7.19.

(Z)-2-Acetamido-5- $N, 6-S$-(N’-2-phenylethyliminomethylidene)-1,2-dideoxy-6-

thionojirimycin Hydrochloride (24). Column chromatography, eluent: 70:10:1 DCM-MeOH$\mathrm{H}_{2} \mathrm{O}$. Yield: $94 \mathrm{mg}$ (quantitative). $[\alpha]_{\mathrm{D}}+2.84$ (c 1.00, MeOH). $\mathrm{R}_{f} 0.30$ (70:10:1 DCM-MeOH-

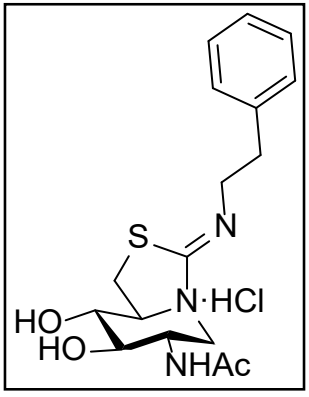
$\left.\mathrm{H}_{2} \mathrm{O}\right)$.

${ }^{1} \mathbf{H}$ NMR (300 MHz, CD $\left.3 \mathrm{OD}\right): \delta$ 7.24-7.09 (m, $\left.4 \mathrm{H}, \mathrm{CH}_{\text {arom }}\right), 3.97(\mathrm{dd}, 1 \mathrm{H}$, $\left.J_{1 \mathrm{a}, 1 \mathrm{~b}}=12.9 \mathrm{~Hz}, J_{1 \mathrm{a}, 2}=5.2 \mathrm{~Hz}, \mathrm{H}-1 \mathrm{a}\right), 3.73(\mathrm{~m}, 1 \mathrm{H}, \mathrm{H}-2), 3.67$ (m, $1 \mathrm{H}, \mathrm{H}-$ 5), $3.48\left(\mathrm{dd}, 1 \mathrm{H}, J_{6 \mathrm{a}, 6 \mathrm{~b}}=11.4 \mathrm{~Hz}, J_{5,6 \mathrm{a}}=7.3 \mathrm{~Hz}, \mathrm{H}-6 \mathrm{a}\right), 3.39$ (m, $3 \mathrm{H}, \mathrm{H}-3$, $\mathrm{CH}_{2} \mathrm{CH}_{2} \mathrm{Ph}$ ), 3.20 (m, $\left.1 \mathrm{H}, \mathrm{H}-4, \mathrm{H}-6 \mathrm{~b}\right), 2.80$ (m, $3 \mathrm{H}, \mathrm{CH}_{2} \mathrm{CH}_{2} \mathrm{Ph}, \mathrm{H}-1 \mathrm{~b}$ ), 1.90 (s, $\left.3 \mathrm{H}, \mathrm{NHCOCH}_{3}\right) .{ }^{13} \mathrm{C}$ NMR $\left(75.5 \mathrm{MHz}, \mathrm{CD}_{3} \mathrm{OD}\right): \delta 172.4(\mathrm{CO})$, $168.1(\mathrm{CN}), 138.0,128.7,128.3,126.3$ (C-arom), 74.4 (C-3), 73.1 (C-4), 66.8 (C-5), 52.2 $\left(\mathrm{CH}_{2} \mathrm{CH}_{2} \mathrm{Ph}\right), 49.6(\mathrm{C}-2), 45.5(\mathrm{C}-1), 35.5(\mathrm{C}-6), 30.1\left(\mathrm{CH}_{2} \mathrm{CH}_{2} \mathrm{Ph}\right), 21.3\left(\mathrm{NHCOCH}_{3}\right)$. ESIMS: $m / z 372.1[\mathrm{M}+\mathrm{Na}]^{+}, 350.1[\mathrm{M}+\mathrm{H}]^{+}$. Anal. Calcd for $\mathrm{C}_{17} \mathrm{H}_{23} \mathrm{~N}_{3} \mathrm{O}_{3} \mathrm{~S} . \mathrm{HCl}$ : C 52.91, H 6.27, N 10.89, S 8.31. Found: C, 52.56; H, 6.08; N, 10.55; S, 7.99.

\section{Synthesis of DNJC-thiazolidines 28-32}

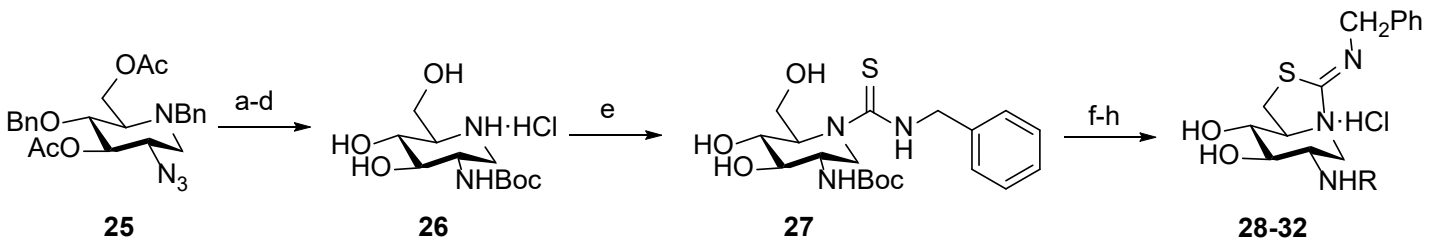


Supplemental scheme 6. Reagents and conditions: a. NaOMe, MeOH, RT, 18 h; b. $\mathrm{PPh}_{3}$, THF$\mathrm{NH}_{4} \mathrm{OH}, 60^{\circ} \mathrm{C}, 18$ h; c. $\mathrm{Boc}_{2} \mathrm{O}, \mathrm{Et}_{3} \mathrm{~N}$, dioxane, RT, 18 h; d. $\mathrm{H}_{2}$, Pd/C, MeOH, RT, 18 h; e. BnNCS, $\mathrm{Et}_{3} \mathrm{~N}, \mathrm{MeCN}, \mathrm{RT}, 18$ h; f. HCl, MeOH, RT, 18 h; g. TFA, 1:1 DCM-H ${ }_{2} \mathrm{O}, \mathrm{RT}, 18$ h. RCOCl or RNCS, $\mathrm{Et}_{3} \mathrm{~N}, \mathrm{MeOH}, \mathrm{RT}, 18$ h, quant.

2-tert-Butoxycarbonylamino-1,2-dideoxynojirimycin (26). 2-Azido-3,6-di- $O$-acetyl-4-Obenzyl-1,2,5-trideoxy-1,5-imino-D-glucitol ${ }^{1} \mathbf{2 5}(207 \mathrm{mg}, 0.45 \mathrm{mM})$ was dissolved in $\mathrm{MeOH}$ (4.5 $\mathrm{mL}$ ) and $\mathrm{MeONa}(5 \mathrm{mg}, 0.09 \mathrm{mM}$ ) was added. The mixture was stirred overnight at RT. Then, dry ice was added until neutral $\mathrm{pH}$ and the solvent was evaporated. The deacetylated product was dissolved in THF-NH $4 \mathrm{OH}(3: 1,4 \mathrm{~mL})$ and reduced with $\mathrm{PPh}_{3}(192 \mathrm{mg}, 0.73 \mathrm{mM})$ overnight at 60 ${ }^{\circ} \mathrm{C}$. The solvent was evaporated and the crude amine was dissolved in dioxane $(4.8 \mathrm{~mL})$. $\mathrm{Boc}_{2} \mathrm{O}$ (213 mg, $0.978 \mathrm{mM})$ and $\mathrm{Et}_{3} \mathrm{~N}(131 \mu \mathrm{L}, 0.978 \mathrm{mM})$ were added and the mixture stirred overnight. The reaction mixture was concetrated to dryness, the crude Boc-protected derivative was dissolved in $\mathrm{MeOH}(4.5 \mathrm{~mL})$ and debenzymated by treatment with $\mathrm{Pd} / \mathrm{C} 10 \%(50 \mathrm{mg})$ overnight under $\mathrm{H}_{2}$ atmosphere. The mixture was filtered over celite, evaporated and suspended in $\mathrm{H}_{2} \mathrm{O}(10$ $\mathrm{mL}$ ). The insoluble $\mathrm{PPh}_{3} \mathrm{O}$ precipitate was filtered and the aqueous solution was lyophilized to give 26. Yield: $58 \mathrm{mg}\left(49 \%, 4\right.$ steps). $\mathrm{R}_{f} 0.30$ (6:3:1 $\left.\mathrm{MeCN}-\mathrm{H}_{2} \mathrm{O}-\mathrm{NH}_{4} \mathrm{OH}\right)$.

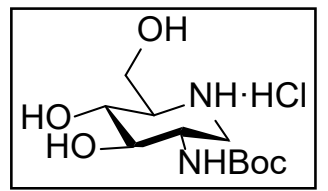

${ }^{1} \mathbf{H}$ NMR (500 MHz, CD $\left.{ }_{3} \mathrm{OD}\right): \delta 3.93\left(\mathrm{dd}, 1 \mathrm{H}, J_{6 \mathrm{a}, 6 \mathrm{~b}}=11.8 \mathrm{~Hz}, J_{5,6 \mathrm{a}}=2.8\right.$ Hz, H-6a), 3.86 (dd, 1 H, $J_{5,6 \mathrm{~b}}=5.3 \mathrm{~Hz}, \mathrm{H}-6 \mathrm{~b}$ ), 3.69 (m, $\left.1 \mathrm{H}, \mathrm{H}-2\right), 3.55$ (bt, $\left.1 \mathrm{H}, J_{3,4}=9.6 \mathrm{~Hz}, \mathrm{H}-4\right), 3.45$ (bt, $\left.1 \mathrm{H}, \mathrm{H}-3\right), 3.39$ (dd, $1 \mathrm{H}, J_{1 \mathrm{a}, 1 \mathrm{~b}}=12.2, J_{1 \mathrm{a}, 2}$ = $4.7 \mathrm{~Hz}, \mathrm{H}-1 \mathrm{a}), 3.07$ (m, $1 \mathrm{H}, \mathrm{H}-5), 2.89$ (bt, $\left.1 \mathrm{H}, J_{1 \mathrm{~b}, 2}=12 \mathrm{~Hz}, \mathrm{H}-1 \mathrm{~b}\right)$, 1.46 (s, $\left.9 \mathrm{H}, \mathrm{COCMe}_{3}\right) .{ }^{13} \mathrm{C}$ NMR (125.7 MHz, $\left.\mathrm{CD}_{3} \mathrm{OD}\right): \delta 156.5(\mathrm{CO}), 79.3\left(\mathrm{CMe}_{3}\right), 73.8(\mathrm{C}-3)$, 68.9 (C-4), 60.5 (C-5), 57.7 (C-6), 49.7 (C-2), 44.8 (C-1), 27.2 (CMes). HRMS (ESI) $m / z$ [M + $\mathrm{H}]^{+}$calcd for $\left[\mathrm{C}_{11} \mathrm{H}_{22} \mathrm{~N}_{2} \mathrm{O}_{5}\right]^{+}$263.1601; found 263.1605.

\section{2-tert-Butoxycarbonylamino-5- $N$-(N'-methylbenzylthiocarbamoyl)-1,2-dideoxynojirimicin}

(27). Compound $26(58 \mathrm{mg}, 0.22 \mathrm{mM})$ in $\mathrm{MeCN}(2.2 \mathrm{~mL})$ was treated with $\mathrm{Et}_{3} \mathrm{~N}(62 \mu \mathrm{L}, 0.44$ mmol) and benzyl isothiocyanate $(44 \mu \mathrm{L}, 0.331 \mathrm{mM})$. The mixture was stirred at RT overnight and concentrated to dryness. The crude product was purified by column cromatography using 100:10:1 DCM-MeOH-H ${ }_{2} \mathrm{O}$ as eluent. Yield: $47 \mathrm{mg}$ (52\%). $\mathrm{R}_{f} 0.40$ (70:10:1 DCM-MeOH-H $\left.\mathrm{H}_{2} \mathrm{O}\right)$.

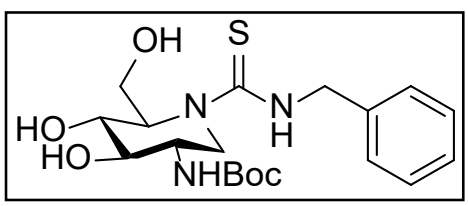

${ }^{1} \mathbf{H}$ NMR $\left(600 \mathrm{MHz}, \mathrm{CD}_{3} \mathrm{OD}\right): \delta$ 7.36-7.22 $\left(\mathrm{CH}_{\text {arom }}\right), 4.90(\mathrm{~m}, 2$ $\left.\mathrm{H}, \mathrm{H}-1 \mathrm{a}, \mathrm{CH}_{2} \mathrm{Ph}\right), 4.80\left(\mathrm{~d}, 1 \mathrm{H},{ }^{2} J_{\mathrm{H}, \mathrm{H}}=15 \mathrm{~Hz}, \mathrm{CHPh}\right), 4.49$ (m, 1 $\mathrm{H}, \mathrm{H}-5), 3.97$ (dd, $\left.1 \mathrm{H}, J_{6 \mathrm{a}, 6 \mathrm{~b}}=11.4 \mathrm{~Hz}, J_{5,6 \mathrm{a}}=8.7 \mathrm{~Hz}, \mathrm{H}-6 \mathrm{a}\right), 3.82$ $\left(\mathrm{dd}, 1 \mathrm{H}, J_{5,6 \mathrm{~b}}=3.6 \mathrm{~Hz}, \mathrm{H}-6 \mathrm{~b}\right), 3.73$ (t, $\left.1 \mathrm{H}, J_{3,4}=J_{4,5}=5.1 \mathrm{~Hz}, \mathrm{H}-4\right), 3.66$ (m, $\left.1 \mathrm{H}, \mathrm{H}-2\right), 3.61$ (t, $\left.1 \mathrm{H}, J_{2,3}=5.1 \mathrm{~Hz}, \mathrm{H}-3\right), 3.52\left(\mathrm{dd}, 1 \mathrm{H}, J_{1 \mathrm{a}, 1 \mathrm{~b}}=14.3 \mathrm{~Hz}, J_{1 \mathrm{~b}, 2}=3.8 \mathrm{~Hz}, \mathrm{H}-1 \mathrm{~b}\right), 1.45\left(\mathrm{~s}, 9 \mathrm{H}, \mathrm{CO}_{2} \mathrm{Me}_{3}\right)$. ${ }^{13}$ C NMR (150 MHz, $\left.\mathrm{CD}_{3} \mathrm{OD}\right): \delta 185.5$ (CS), $156.3(\mathrm{CO}), 138.7,128.0,127.2,126.6\left(\mathrm{C}_{\text {arom }}\right), 79.0$ 
(CMe 3$), 70.8$ (C-3), 69.2 (C-4), 64.3 (C-5), 60.4 (C-6), 52.0 (C-2), $49.2\left(\mathrm{CH}_{2} \mathrm{Ph}\right), 43.9(\mathrm{C}-1), 27.3$ $\left(\mathrm{CMe} e_{3}\right.$ ). HRMS (ESI) $m / z[\mathrm{M}+\mathrm{H}]^{+}$calcd for $\left[\mathrm{C}_{19} \mathrm{H}_{29} \mathrm{~N}_{3} \mathrm{O}_{5} \mathrm{~S}\right]^{+} 412.1901$; found 412.1904 .

General procedure for the preparation of the DNJC-thiazolidines 28-32. Compound 27 (47 $\mathrm{mg}, 0.11 \mathrm{mM})$ was dissolved in $\mathrm{MeOH}(2 \mathrm{~mL})$ and concentrated $\mathrm{HCl}$ was dropwise added until $\mathrm{pH}$ 1. The mixture was stirred at rt overnight and monitored by ESIMS to confim total conversion into the corresponding cyclic isourea. The reaction mixture was concentrated and the crude product was dissolved in 1:1 DCM- $\mathrm{H}_{2} \mathrm{O}(2 \mathrm{~mL})$. TFA ( $\left.30 \mu \mathrm{L}, 0.38 \mathrm{mM}, 3 \mathrm{eq}\right)$ was then added and the solution was stirred overnight. The mixture was evaported, co-evaporated with toluene (3 $\mathrm{x} 5 \mathrm{~mL}$ ) and lyophilized. The free amine derivative thus obtained was engaged in the nex reactions without further purification. Thus, $10 \mathrm{mg}(0.034 \mathrm{mM})$ of this material was dissolved in $\mathrm{MeOH}(3$ $\mathrm{mL}$ ) and reacted with either propionyl, butyryl or isobutyryl chorideor ethyl or propyl isothiocyanate $(1.2 \mathrm{eq})$ in the presence of $\mathrm{Et}_{3} \mathrm{~N}(3 \mathrm{eq})$. The reaction mixtures were stirred at RT overnight, the solvent was evaporated and the crude product was purified as indicated for each compound.

\section{(Z)-2-Propionamido-5- $N, 6-S$-( $N$ '-benzyliminomethylidene)-1,2-dideoxy-6-thionojirimycin}

Hydrochloride (28). Column chromatography, eluent: 70:10:1 DCM-MeOH- $\mathrm{H}_{2} \mathrm{O}$. Yield: $94 \mathrm{mg}$ (quantitative). $[\alpha]_{\mathrm{D}}+31.9$ (c 1.00, MeOH). $\mathrm{R}_{f} 0.30$ (70:10:1 DCM-MeOH-H $\left.2 \mathrm{O}\right)$.

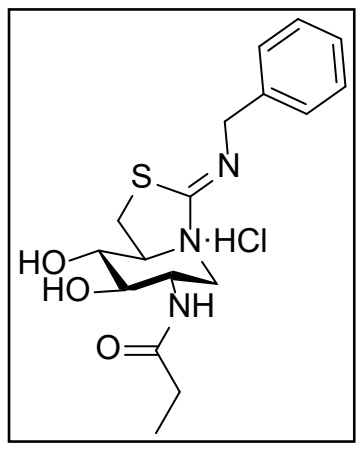

${ }^{1}$ H NMR (500 MHz, $\left.\mathrm{CD}_{3} \mathrm{OD}\right): \delta$ 7.18-7.08 (m, $\left.5 \mathrm{H}, \mathrm{CH}_{\text {arom }}\right), 4.26(\mathrm{~d}, 2$ $\left.\mathrm{H},{ }^{3} J_{H, H}=15 \mathrm{~Hz}, \mathrm{CH}_{2} \mathrm{Ph}\right), 4.05\left(\mathrm{dd}, 1 \mathrm{H}, J_{1 \mathrm{a}, 1 \mathrm{~b}}=12.6 \mathrm{~Hz}, J_{1 \mathrm{a}, 2}=5.3 \mathrm{~Hz}\right.$, H-1a), 3.75 (m, $1 \mathrm{H}, \mathrm{H}-2), 3.36$ (dd, $1 \mathrm{H}, J_{6 \mathrm{a}, 6 \mathrm{~b}}=11.2 \mathrm{~Hz}, J_{5,6 \mathrm{a}}=7.3 \mathrm{~Hz}$, H-6a), 3.29 (m, 1 H, H-5), 3.23 (m, 2 H, H-3, H-4), 3.08 (dd, 1 H, H-6b), $2.51\left(\mathrm{dd}, 1 \mathrm{H}, J_{1 \mathrm{~b}, 2}=11.7 \mathrm{~Hz}, \mathrm{H}-1 \mathrm{~b}\right), 2.13\left(\mathrm{q}, 2 \mathrm{H}, \mathrm{COCH}_{2} \mathrm{CH}_{3}\right), 1.03(\mathrm{t}$, $\left.3 \mathrm{H}, \mathrm{COCH}_{2} \mathrm{CH}_{3}\right) .{ }^{13} \mathbf{C}$ NMR (125.7 MHz, $\left.\mathrm{CD}_{3} \mathrm{OD}\right): \delta 177.4(\mathrm{CO}), 163.7$ $(\mathrm{CN}), 141.3,129.3,128.5,127.8\left(\mathrm{C}_{\text {arom }}\right), 77.0$ (C-3), 75.5 (C-4), 66.2 (C5), $58.8\left(\mathrm{CH}_{2} \mathrm{Ph}\right), 51.2(\mathrm{C}-2), 47.3(\mathrm{C}-1), 31.4(\mathrm{C}-6) 30.3\left(\mathrm{COCH}_{2} \mathrm{CH}_{3}\right), 10.3\left(\mathrm{COCH}_{2} \mathrm{CH}_{3}\right)$. ESIMS: $m / z$ 350.1 $[\mathrm{M}+\mathrm{H}]^{+}$. Anal. Calcd for $\mathrm{C}_{17} \mathrm{H}_{23} \mathrm{~N}_{3} \mathrm{O}_{3} \mathrm{~S}$. HCl: C 52.91, H 6.27, N 10.89, S 8.31. Found: C, 52.98; H, 6.40; N, 10.71; S, 8.52. 
(Z)-2-Butyramido-5- $N, 6-S$-( $N$ '-benzyliminomethylidene)-1,2-dideoxy-6-thionojirimycin (29). Column chromatography, eluent: 100:10:1 DCM-MeOH- $\mathrm{H}_{2} \mathrm{O}$. Yield: $98 \mathrm{mg}$ (quantitative). $\mathrm{R}_{f}$ 0.50 (70:10:1 DCM-MeOH-H $\left.\mathrm{H}_{2} \mathrm{O}\right)$.

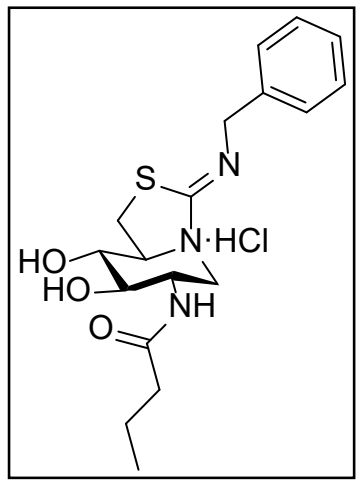

${ }^{1} \mathbf{H}$ NMR (600 MHz, $\left.\mathrm{CD}_{3} \mathrm{OD}\right): \delta$ 7.31-7.20 (m, $\left.5 \mathrm{H}, \mathrm{CH}_{\text {arom }}\right), 4.37(\mathrm{~d}, 2$ $\left.\mathrm{H},{ }^{3} J_{H, H}=15.0 \mathrm{~Hz}, \mathrm{CH}_{2} \mathrm{Ph}\right), 4.16\left(\mathrm{dd}, 1 \mathrm{H}, J_{1 \mathrm{a}, 1 \mathrm{~b}}=12.6 \mathrm{~Hz}, J_{1 \mathrm{a}, 2}=5.3 \mathrm{~Hz}\right.$ , H-1a), 3.88 (m, $1 \mathrm{H}, \mathrm{H}-2), 3.48$ (dd, $1 \mathrm{H}, J_{6 \mathrm{a}, 6 \mathrm{~b}}=11.2 \mathrm{~Hz}, J_{5,6 \mathrm{a}}=7.3 \mathrm{~Hz}$, H-6a), 3.40 (m, 1 H, H-5), 3.37 (m, 2 H, H-3, H-4), 3.19 (dd, 1 H, H6b), 2.61 (dd, $\left.1 \mathrm{H}, J_{1 \mathrm{~b}, 2}=11.2 \mathrm{~Hz}, \mathrm{H}-1 \mathrm{~b}\right), 2.49,1.66$ (m, $4 \mathrm{H}, \mathrm{CH}_{2} \mathrm{CH}_{3}$ ), 0.97 (t, $\left.3 \mathrm{H}, \mathrm{CH}_{2} \mathrm{CH}_{3}\right) .{ }^{13} \mathrm{C}$ NMR (150 MHz, $\left.\mathrm{CD}_{3} \mathrm{OD}\right): \delta 175.0$ (CO), $162.1(\mathrm{CN}), 140.0,127.8,127.1,126.3\left(\mathrm{C}_{\text {arom }}\right), 75.5$ (C-3), $74.2(\mathrm{C}-4)$, 64.7 (C-5), $57.6\left(\mathrm{CH}_{2} \mathrm{Ph}\right), 49.7$ (C-2), $45.8(\mathrm{C}-1), 37.7$ (C-6), 29.9, $18.9\left(\mathrm{CH}_{2} \mathrm{CH}_{3}\right), 12.5$ $\left(\mathrm{CH}_{2} \mathrm{CH}_{3}\right)$. HRMS (ESI) $\mathrm{m} / z[\mathrm{M}+\mathrm{H}]^{+}$calcd for $\left[\mathrm{C}_{18} \mathrm{H}_{25} \mathrm{~N}_{3} \mathrm{O}_{3} \mathrm{~S}\right]^{+}$364.1689; found 364.1696.

\section{2-Isobutyramido-5- $N, 6-S$-( $N$ '-benzyliminomethylidene)-1,2-dideoxy-6-thionojirimycin}

Hydrochloride (30). Column chromatography, eluent: 70:10:1 DCM-MeOH- $\mathrm{H}_{2} \mathrm{O}$. Yield: $98 \mathrm{mg}$ (quantitative). $[\alpha]_{\mathrm{D}}+36.2\left(c\right.$ 1.04, MeOH). $\mathrm{R}_{f} 0.50\left(70: 10: 1 \mathrm{DCM}-\mathrm{MeOH}-\mathrm{H}_{2} \mathrm{O}\right)$.

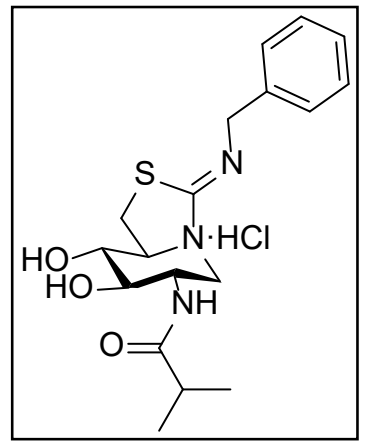

${ }^{1} \mathbf{H}$ NMR (500 MHz, $\left.\mathrm{CD}_{3} \mathrm{OD}\right): \delta$ 7.31-7.20 (m, $\left.5 \mathrm{H}, \mathrm{CH}_{\text {arom }}\right), 4.39(\mathrm{~d}, 2$ $\left.\mathrm{H},{ }^{3} J_{H, H}=15.0 \mathrm{~Hz}, \mathrm{CH}_{2} \mathrm{Ph}\right), 4.17\left(\mathrm{dd}, 1 \mathrm{H}, J_{1 \mathrm{a}, 1 \mathrm{~b}}=12.6 \mathrm{~Hz}, J_{1 \mathrm{a}, 2}=5.3\right.$ Hz, H-1a), 3.87 (m, $1 \mathrm{H}, \mathrm{H}-2), 3.49$ (dd, $1 \mathrm{H}, J_{6 \mathrm{a}, 6 \mathrm{~b}}=11.2 \mathrm{~Hz}, J_{5,6 \mathrm{a}}=7.3$ Hz, H-6a), 3.47 (m, 1 H, H-5), 3.39 (m, 2 H, H-3, H-4), 3.20 (dd, 1 H, $\mathrm{H}-6 \mathrm{~b}), 2.64$ (dd, $\left.1 \mathrm{H}, J_{1 \mathrm{~b}, 2}=11.7 \mathrm{~Hz}, \mathrm{H}-1 \mathrm{~b}\right), 2.49$ (q, $2 \mathrm{H}, \mathrm{COCH}_{2} \mathrm{Me}_{2}$ ), $1.15\left(\mathrm{t}, 6 \mathrm{H}, \mathrm{COCH}_{2} \mathrm{Me}_{2}\right) .{ }^{13} \mathrm{C}$ NMR $\left(125.7 \mathrm{MHz}, \mathrm{CD}_{3} \mathrm{OD}\right): \delta 180.6$ (CO), $163.8(\mathrm{CN}), 141.2,129.3,128.6,127.8$ (C $\mathrm{Carom}), 76.9(\mathrm{C}-3), 75.7$

(C-4), 66.2 (C-5), 58.8 ( $\left.\mathrm{CH}_{2} \mathrm{Ph}\right), 51.1$ (C-2), 47.1 (C-1), 36.4 (C-6) $31.4\left(\mathrm{COCHMe}_{2}\right), 20.0,19.7$ (COCHMe $e_{2}$ ). ESIMS: $m / z 364.1[\mathrm{M}+\mathrm{H}]^{+}$. Anal. Calcd for $\mathrm{C}_{18} \mathrm{H}_{25} \mathrm{~N}_{3} \mathrm{O}_{3} \mathrm{~S} . \mathrm{HCl}$ : C, 54.06; H, 6.55; N, 10.51; S, 8.02. Found: C, 53.79; H, 6.47; N, 10.32; S, 7.72.

(Z)-2- $N$ '-Ethylthioureido-5- $N, 6-S$-( $N$ '-benzyliminomethylidene)-1,2-dideoxy-6thionojirimycin (31). Column chromatography, eluent: 100:10:1 DCM-MeOH- $\mathrm{H}_{2} \mathrm{O}$. Yield: 102 mg (quantitative). $\mathrm{R}_{f} 0.50$ (70:10:1 DCM-MeOH- $\left.\mathrm{H}_{2} \mathrm{O}\right)$. 


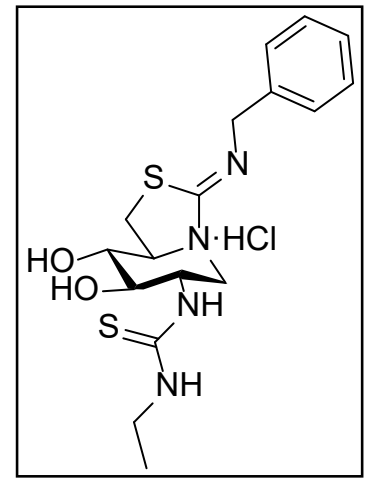

${ }^{1} \mathbf{H}$ NMR (600 MHz, $\left.\mathrm{CD}_{3} \mathrm{OD}\right): \delta$ 7.33-7.21 (m, $\left.5 \mathrm{H}, \mathrm{CH}_{\text {arom }}\right), 4.37$ (d, 3 $\left.\mathrm{H},{ }^{3} \mathrm{~J}_{\mathrm{H}, \mathrm{H}}=15.0 \mathrm{~Hz}, \mathrm{CH}_{2} \mathrm{Ph}, \mathrm{H}-1 \mathrm{a}\right), 3.47$ (m, $\left.3 \mathrm{H}, \mathrm{H}-6 \mathrm{a}, \mathrm{CH}_{2} \mathrm{~N}\right), 3.41(\mathrm{~m}$, 1 H, H-2), 3.37 (m, 3 H, H-3, H-4, H-5), 3.20 (dd, 1 H, H-6b), 2.60 (dd, $\left.1 \mathrm{H}, J_{1 \mathrm{~b}, 2}=11.2 \mathrm{~Hz}, \mathrm{H}-1 \mathrm{~b}\right), 1.16\left(\mathrm{t}, 3 \mathrm{H}, \mathrm{CH}_{2} \mathrm{CH}_{3}\right) .{ }^{13} \mathbf{C} \mathbf{N M R}(150 \mathrm{MHz}$, $\left.\mathrm{CD}_{3} \mathrm{OD}\right): \delta 161.7(\mathrm{CN}), 140.2,127.8,127.1,126.2\left(\mathrm{C}_{\text {arom }}\right), 76.3(\mathrm{C}-3)$, 73.8 (C-4), $64.5(\mathrm{C}-5), 57.7\left(\mathrm{CH}_{2} \mathrm{Ph}\right), 48.1\left(\mathrm{CH}_{2} \mathrm{~N}, \mathrm{C}-2\right), 45.7$ (C-1), 29.7 (C-6), $13.2\left(\mathrm{CH}_{2} \mathrm{CH}_{3}\right)$. HRMS (ESI) $\mathrm{m} / z[\mathrm{M}+\mathrm{H}]^{+}$calcd for $\left[\mathrm{C}_{17} \mathrm{H}_{24} \mathrm{~N}_{4} \mathrm{O}_{2} \mathrm{~S}_{2}\right]^{+}$381.1413; found 381.1424 .

\section{(Z)-2-Propylthioureido-5- $N, 6-S$-( $N$ '-benzyliminomethylidene)-1,2-dideoxy-6-}

thionojirimycin (32). Column chromatography, eluent: 100:10:1 DCM-MeOH- $\mathrm{H}_{2} \mathrm{O}$. Yield: 106 $\mathrm{mg}$ (quantitative). $\mathrm{R}_{f} 0.51$ (70:10:1 DCM-MeOH-H $2 \mathrm{O}$ ).

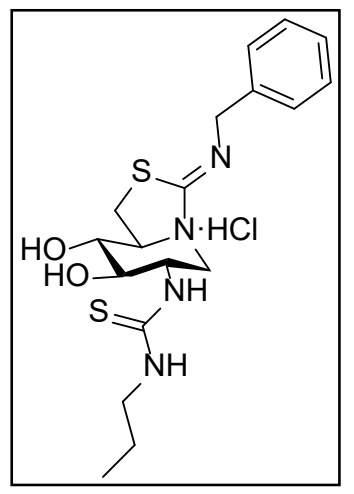

${ }^{1}$ H NMR (600 MHz, $\left.\mathrm{CD}_{3} \mathrm{OD}\right): \delta$ 7.33-7.20 (m, $\left.5 \mathrm{H}, \mathrm{CH}_{\text {arom }}\right), 4.38(\mathrm{~d}, 3$ $\left.\mathrm{H},{ }^{3} J_{H, H}=15.0 \mathrm{~Hz}, \mathrm{CH}_{2} \mathrm{Ph}, \mathrm{H}-1 \mathrm{a}\right), 3.48\left(\mathrm{~m}, 3 \mathrm{H}, \mathrm{H}-6 \mathrm{a}, \mathrm{CH}_{2} \mathrm{~N}\right), 3.42(\mathrm{~m}$, 1 H, H-2), 3.37 (m, 3 H, H-3, H-4, H-5), 3.21 (dd, 1 H, H-6b), 2.62 (dd, $\left.1 \mathrm{H}, J_{1 \mathrm{~b}, 2}=11.2 \mathrm{~Hz}, \mathrm{H}-1 \mathrm{~b}\right), 1.58\left(\mathrm{t}, 3 \mathrm{H}, \mathrm{CH}_{2} \mathrm{CH}_{3}\right), 0.94\left(\mathrm{t}, 3 \mathrm{H}, \mathrm{CH}_{2} \mathrm{CH}_{3}\right)$. ${ }^{13} \mathrm{C}$ NMR (150 MHz, $\left.\mathrm{CD}_{3} \mathrm{OD}\right): \delta 162.0(\mathrm{CN}), 140.0,127.8,127.1,126.3$ ( $\left.\mathrm{C}_{\text {arom}}\right), 76.3(\mathrm{C}-3), 73.8(\mathrm{C}-4), 64.5(\mathrm{C}-5), 57.8\left(\mathrm{CH}_{2} \mathrm{Ph}\right), 48.1\left(\mathrm{CH}_{2} \mathrm{~N}\right.$, C-2), 45.7 (C-1), 29.7 (C-6), $21.9\left(\mathrm{CH}_{2} \mathrm{CH}_{3}\right), 10.2\left(\mathrm{CH}_{2} \mathrm{CH}_{3}\right)$. HRMS (ESI) $m / z[\mathrm{M}+\mathrm{H}]^{+}$calcd for $\left[\mathrm{C}_{18} \mathrm{H}_{26} \mathrm{~N}_{4} \mathrm{O}_{2} \mathrm{~S}_{2}\right]^{+}$395.1570; found 395.1567 . 


\section{Synthesis of DNJC-thiazolidines 37 and 38.}

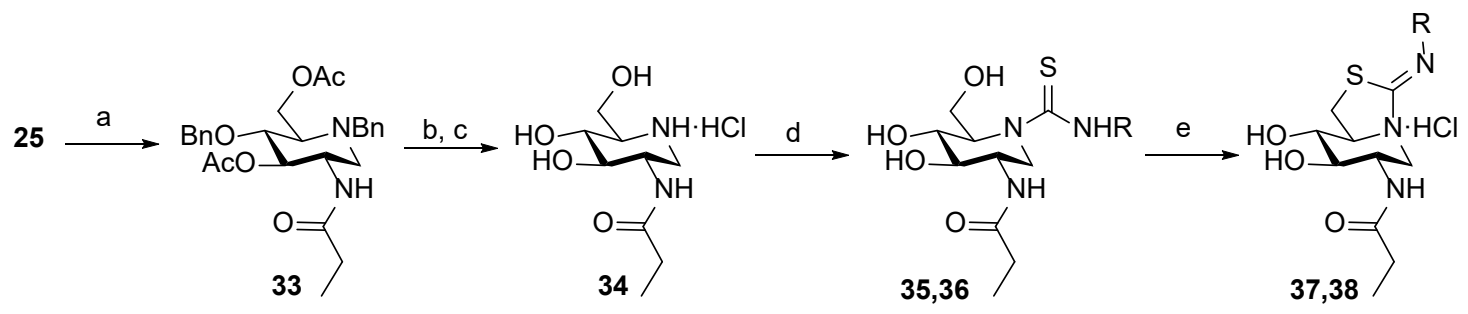

Supplemental scheme 7. Reagents and conditions: a. $\mathrm{Zn}, \mathrm{CuSO}_{4(\mathrm{aq})}$, THF-PrOH-Pr $2 \mathrm{O}, \mathrm{RT}, 20$ min; b. NaOMe, MeOH, RT, 18 h; c. $\mathrm{H}_{2}, \mathrm{Pd} / \mathrm{C}, \mathrm{MeOH}, \mathrm{RT}, 18$ h; d. R-NCS, Et 3 N, MeCN, RT, 18 h; e. $\mathrm{HCl}, \mathrm{MeOH}, \mathrm{RT}, 18$ h.

\section{2-Propionamide-3,6-di- $O$-acetyl-1- $N, 4-O$-dibenzyl-1,2-dideoxynojirimycin}

Powdered zinc ( $373 \mathrm{mg}, 5.74 \mathrm{mM}$ ) was added to a solution of the azide $\mathbf{2 5}^{1}(130 \mathrm{mg}, 0.28 \mathrm{mM})$ in 3:2:1 THF-propionic acid-propionic anhydride $(3.5 \mathrm{~mL})$ and stirred vigorously before the dropwise addition of a saturated copper (II) sulfate solution $(0.9 \mathrm{~mL})$ to initiate the reaction. After 20 min, t.l.c. analysis (1:2 EtOAc-CyHex) indicated the complete consumption of the starting material $\left(R_{f} 0.50\right)$ and the formation of a major product $\left(R_{f} 0.10\right)$. The reaction mixture was filtered through Celite $^{\circledR}$, concentrated in vacuo and the crude residue purified by flash chromatography (50:1, DCM-MeOH) to afford the propanamide $33(109 \mathrm{mg}, 79 \%)$. $[\alpha]_{\mathrm{D}}+8.80$ (c 1, MeOH). $\mathrm{R}_{f} 0.50$ (20:1 DCM-MeOH).

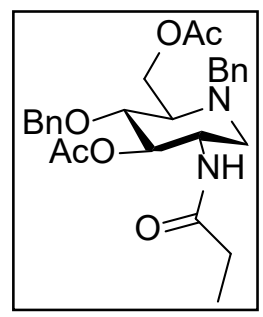

${ }^{1} \mathbf{H}$ NMR (300 MHz, $\left.\mathrm{CDCl}_{3}\right): \delta 7.36-7.23\left(\mathrm{~m}, 10 \mathrm{H}, \mathrm{CH}_{\text {arom }}\right), 6.07(\mathrm{~s}, 1 \mathrm{H}$, $\left.J_{\mathrm{NH}, 2}=8.7 \mathrm{~Hz}, \mathrm{NH}\right), 4.90\left(\mathrm{dd}, 1 \mathrm{H}, J_{2,3}=J_{3,4}=8.6 \mathrm{~Hz}, \mathrm{H}-3\right), 4.65(\mathrm{dd}, 2 \mathrm{H}$, $\left.{ }^{2} J_{\mathrm{H}, \mathrm{H}}=11.1 \mathrm{~Hz}, \mathrm{OCH} \mathrm{H}_{2} \mathrm{Ph}\right), 4.51\left(\mathrm{dd}, 1 \mathrm{H}, J_{6 \mathrm{a}, 6 \mathrm{~b}}=12.1 \mathrm{~Hz}, J_{5,6 \mathrm{a}}=J_{5,6 \mathrm{~b}}=4.1\right.$ Hz, H-6a), 4.37 (dd, 1 H, H-6b), 4.15 (m, 1 H, H-2), 3.92 (dd, 2 H, $J_{\text {gem }}=$ $\left.13.6 \mathrm{~Hz}, \mathrm{NCH}_{2} \mathrm{Ph}\right), 3.73\left(\mathrm{t}, 1 \mathrm{H}, J_{4,3}=J_{4,5}=6.7 \mathrm{~Hz}, \mathrm{H}-4\right), 3.05(\mathrm{dd}, 1 \mathrm{H}$, $\left.J_{1 \mathrm{a}, 1 \mathrm{~b}}=12.0 \mathrm{~Hz}, J_{1 \mathrm{a}, 2}=3.7 \mathrm{~Hz}, \mathrm{H}-1 \mathrm{a}\right), 2.86(\mathrm{~m}, 1 \mathrm{H}, \mathrm{H}-5), 2.18$ (dd, $\left.1 \mathrm{H}, J_{1 \mathrm{~b}, 2}=7.8 \mathrm{~Hz}, \mathrm{H}-1 \mathrm{~b}\right)$, $2.05\left(\mathrm{~m}, 2 \mathrm{H}, \mathrm{COCH}_{2} \mathrm{CH}_{3}\right), 2.04\left(\mathrm{~s}, 6 \mathrm{H}, \mathrm{COCH}_{3}\right), 1.02$ (t, $\left.3 \mathrm{H}, \mathrm{COCH}_{2} \mathrm{CH}_{3}\right) .{ }^{13} \mathbf{C ~ N M R}(75.5$ $\left.\mathrm{MHz}, \mathrm{CDCl}_{3}\right): \delta 173.1,170.9,170.7\left(\mathrm{COCH}_{3}, \mathrm{NHCOCH}_{2}\right), 138.1-127.3\left(\mathrm{C}_{\text {arom }}\right), 75.5(\mathrm{C}-4), 74.4$ (C-3), $73.9\left(\mathrm{OCH}_{2} \mathrm{Ph}\right), 61.9$ (C-5), 59.7 (C-6), $57.3\left(\mathrm{NCH}_{2} \mathrm{Ph}\right), 51.6(\mathrm{C}-1), 47.9$ (C-2), 29.7 $\left(\mathrm{COCH}_{2} \mathrm{CH}_{3}\right), 20.9,20.8\left(\mathrm{COCH}_{3}\right), 9.5\left(\mathrm{COCH}_{2} \mathrm{CH}_{3}\right)$. ESIMS: $483.3[\mathrm{M}+\mathrm{H}]^{+}, 505.3[\mathrm{M}+\mathrm{Na}]^{+}$. Anal. Calcd for $\mathrm{C}_{27} \mathrm{H}_{34} \mathrm{~N}_{2} \mathrm{O}_{6}$ : C 67.20, H 7.10, N 5.81. Found: C, 67.33; H, 7.26; N, 5.74. 
Column chromatography, eluent 100:10:1 DCM-MeOH- $\mathrm{H}_{2} \mathrm{O}$. Yield: $90 \mathrm{mg}(60 \%)$. $\mathrm{R}_{f} 0.40$ (70:10:1 DCM-MeOH-H $\mathrm{H}_{2} \mathrm{O}$ ).

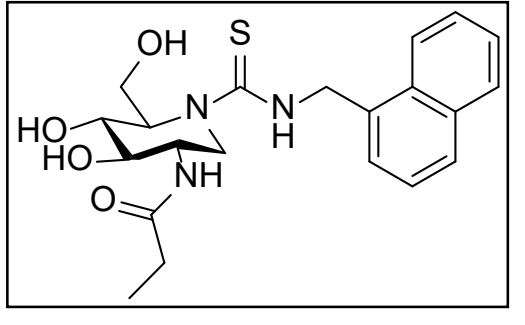

${ }^{1} \mathbf{H}$ NMR (600 MHz, $\left.\mathrm{CD}_{3} \mathrm{OD}\right): \delta 8.10-7.42\left(\mathrm{~m}, 8 \mathrm{H}, \mathrm{CH}_{\text {arom }}\right)$, 5.27 (bs, $2 \mathrm{H}, \mathrm{CH}_{2} \mathrm{Naph}$ ), 4.98 (m, $\left.1 \mathrm{H}, \mathrm{H}-1 \mathrm{a}\right), 4.31$ (m, $1 \mathrm{H}, \mathrm{H}-$ 5), $3.95\left(\mathrm{dd}, 1 \mathrm{H}, J_{6 \mathrm{a}, 6 \mathrm{~b}}=11.2 \mathrm{~Hz}, J_{5,6 \mathrm{a}}=8.8 \mathrm{~Hz}, \mathrm{H}-6 \mathrm{a}\right), 3.95$ (m, $1 \mathrm{H}, \mathrm{H}-2), 3.75$ (dd, $\left.1 \mathrm{H}, J_{5,6 \mathrm{~b}}=3.5 \mathrm{~Hz}, \mathrm{H}-6 \mathrm{~b}\right), 3.67$ (t, $1 \mathrm{H}$, $\left.J_{3,4}=J_{4,5}=5.7 \mathrm{~Hz}, \mathrm{H}-4\right), 3.62\left(\mathrm{t}, 1 \mathrm{H}, J_{2,3}=5.4 \mathrm{~Hz}, \mathrm{H}-3\right), 3.54$ $\left(\mathrm{dd}, 1 \mathrm{H}, J_{1 \mathrm{a}, \mathrm{b}}=14.1 \mathrm{~Hz}, J_{1 \mathrm{~b}, 2}=3.8 \mathrm{~Hz}, \mathrm{H}-1 \mathrm{~b}\right), 2.22\left(\mathrm{q}, 2 \mathrm{H}, \mathrm{CH}_{2} \mathrm{CH}_{3}\right), 1.14$ (t, $\left.3 \mathrm{H}, \mathrm{CH}_{2} \mathrm{CH}_{3}\right)$. ${ }^{13}$ C NMR (150 MHz, $\left.\mathrm{CD}_{3} \mathrm{OD}\right): \delta 185.4$ (CS), 175.1 (CO), 133.9-123.2 (C $\left.\mathrm{C}_{\text {arom }}\right), 70.7$ (C-3), 69.1 (C-4), 64.8 (C-5), 60.3 (C-6), 51.3 (C-2, $\left.\mathrm{CH}_{2} \mathrm{Naph}\right), 43.6$ (C-1), $29.1\left(\mathrm{CH}_{2} \mathrm{CH}_{3}\right), 8.6\left(\mathrm{CH}_{2} \mathrm{CH}_{3}\right)$. HRMS (ESI) $m / z[\mathrm{M}+\mathrm{Na}]^{+}$calcd for $\left[\mathrm{C}_{21} \mathrm{H}_{27} \mathrm{~N}_{3} \mathrm{NaO}_{4} \mathrm{~S}\right]^{+} 440.1614$; found 440.1607

\section{2-Propionamido-5- $N$-(N'-(1-Boc-indol-5-ylmethyl)thiocarbamoyl)-1,2-dideoxynojirimycin}

(36). Column chromatography, eluent 100:10:1 DCM-MeOH-H ${ }_{2} \mathrm{O}$. Yield: $90 \mathrm{mg}(65 \%)$. $[\alpha]_{\mathrm{D}}$ 54.6 (c 1.0, MeOH). $\mathrm{R}_{f} 0.60$ (70:10:1 DCM-MeOH-H $\left.\mathrm{H}_{2} \mathrm{O}\right)$.

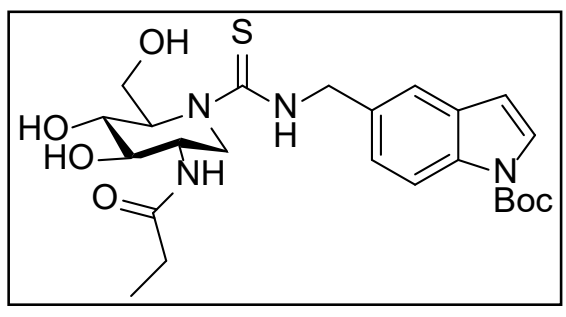

${ }^{1}$ H NMR $\left(500 \mathrm{MHz}, \mathrm{CD}_{3} \mathrm{OD}\right): \delta 8.04-6.55(\mathrm{~m}, 5 \mathrm{H}$, $\left.\mathrm{CH}_{\text {arom }}\right), 4.88\left(\mathrm{~d}, 3 \mathrm{H},{ }^{3} J_{H, H}=15.0 \mathrm{~Hz}, \mathrm{CH}_{2} \mathrm{Ph}\right), 4.84$ (m, 1 H, H-1a), 4.40 (m, $1 \mathrm{H}, \mathrm{H}-5), 3.95$ (dd, $1 \mathrm{H}, J_{6 \mathrm{a}, 6 \mathrm{~b}}=11.2$ $\left.\mathrm{Hz}, J_{5,6 \mathrm{a}}=8.8 \mathrm{~Hz}, \mathrm{H}-6 \mathrm{a}\right), 3.92$ (m, $\left.1 \mathrm{H}, \mathrm{H}-2\right), 3.80$ (dd, $1 \mathrm{H}$, $\left.J_{5,6 \mathrm{~b}}=3.6 \mathrm{~Hz}, \mathrm{H}-6 \mathrm{~b}\right), 3.69\left(\mathrm{t}, 1 \mathrm{H}, J_{3,4}=J_{4,5}=5.2 \mathrm{~Hz}, \mathrm{H}-4\right)$, $3.60\left(\mathrm{t}, 1 \mathrm{H}, J_{2,3}=5.4 \mathrm{~Hz}, \mathrm{H}-3\right), 3.51\left(\mathrm{dd}, 1 \mathrm{H}, J_{1 \mathrm{a}, 1 \mathrm{~b}}=14.3 \mathrm{~Hz}, J_{1 \mathrm{~b}, 2}=3.6 \mathrm{~Hz}, \mathrm{H}-1 \mathrm{~b}\right), 2.17$ (q, 2 $\left.\mathrm{H}, \mathrm{CH}_{2} \mathrm{CH}_{3}\right), 1.68$ (s, $\left.9 \mathrm{H}, \mathrm{CO}_{2} \mathrm{Me}_{3}\right), 1.07$ (t, $\left.3 \mathrm{H}, \mathrm{CH}_{2} \mathrm{CH}_{3}\right) .{ }^{13} \mathbf{C}$ NMR (125.7 MHz, CD $\left.{ }_{3} \mathrm{OD}\right): \delta$ 185.4 (CS), 175.1 ( $\left.\mathrm{CO}_{\text {amide }}\right), 149.6\left(\mathrm{CO}_{\text {carbamate }}\right), 149.6-106.9\left(\mathrm{C}_{\text {arom }}\right), 83.5\left(\mathrm{CCH}_{3}\right), 70.7(\mathrm{C}-3), 69.2$ (C-4), 64.6 (C-5), 60.2 (C-6), $51.3(\mathrm{C}-2), 49.2\left(\mathrm{CH}_{2} \mathrm{Ph}\right), 43.6(\mathrm{C}-1), 29.1\left(\mathrm{CH}_{2} \mathrm{CH}_{3}\right), 26.9$ $\left(\mathrm{CO}_{2} \mathrm{Me}_{3}\right), 8.6\left(\mathrm{CH}_{2} \mathrm{CH}_{3}\right)$. HRMS (ESI) $\mathrm{m} / z[\mathrm{M}+\mathrm{Na}]^{+}$calcd for $\left[\mathrm{C}_{24} \mathrm{H}_{34} \mathrm{~N}_{4} \mathrm{NaO}_{6} \mathrm{~S}\right]^{+}$529.2091; found 529.2082

(Z)-2-Propionamido-5- $N, 6-S$-( $N$ '-1-naphthymethyliminomethylidene)-1,2-dideoxy-6-

thionojirimycin Hydrochloride (37). Column chromatography, eluent: 70:10:1 DCM-MeOH$\mathrm{H}_{2} \mathrm{O}$. Yield: $104 \mathrm{mg}$ (quantitative). $\mathrm{R}_{f} 0.62$ (50:10:1 DCM-MeOH- $\mathrm{H}_{2} \mathrm{O}$ ).

${ }^{1}$ H NMR (500 MHz, 1:1 CD $\mathrm{CDD}_{3} \mathrm{ODCCl}$ ): $\delta$ 8.03-7.43 (m, $8 \mathrm{H}, \mathrm{CH}_{\text {arom }}$ ), 4.90 (bs, 2H, $\mathrm{CH}_{2} \mathrm{Naph}$ ), $4.22\left(\mathrm{dd}, 1 \mathrm{H}, J_{1 \mathrm{a}, \mathrm{b}}=13.1 \mathrm{~Hz}, J_{\mathrm{la}, 2}=5.2 \mathrm{~Hz}, \mathrm{H}-1 \mathrm{a}\right), 3.86$ (m, $\left.1 \mathrm{H}, \mathrm{H}-2\right), 3.65$ (m, 1 H, H-5), 3.58 


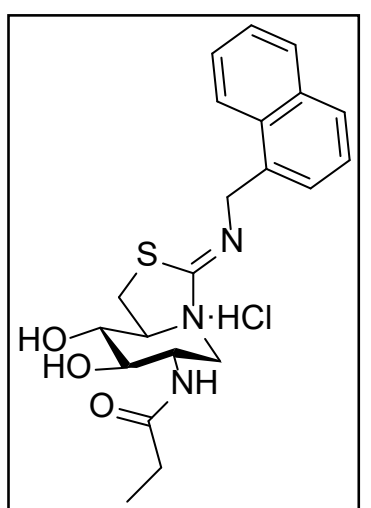

H, 5.82; N, 9.30; S, 7.03.

(m, $1 \mathrm{H}, \mathrm{H}-6 \mathrm{a}), 3.47$ (t, $\left.1 \mathrm{H}, J_{2,3}=J_{3,4}=9.9, \mathrm{H}-3\right), 3.40$ (t, $\left.1 \mathrm{H}, \mathrm{H}-4\right)$, 3.33 (m, $1 \mathrm{H}, \mathrm{H}-6 \mathrm{~b}), 2.78$ (dd, $\left.1 \mathrm{H}, J_{1 \mathrm{~b}, 2}=11.5 \mathrm{~Hz}, \mathrm{H}-1 \mathrm{~b}\right), 2.25$ (q, 2 $\left.\mathrm{H}, \mathrm{CH}_{2} \mathrm{CH}_{3}\right), 1.14\left(\mathrm{t}, 3 \mathrm{H}, \mathrm{CH}_{2} \mathrm{CH}_{3}\right) .{ }^{13} \mathbf{C}$ NMR $(125.7 \mathrm{MHz}, 1: 1$ $\left.\mathrm{CD}_{3} \mathrm{OD}-\mathrm{CDCl}_{3}\right): \delta 176.3(\mathrm{CO}), 165.1(\mathrm{CN}), 133.8-122.9\left(\mathrm{C}_{\text {arom }}\right), 75.1$ (C-3), 73.8 (C-4), 65.8 (C-5), 53.6 ( $\left.\mathrm{CH}_{2} \mathrm{Naph}\right), 49.8$ (C-2), 45.9 (C-1), 30.5 (C-6), $29.0\left(\mathrm{CH}_{2} \mathrm{CH}_{3}\right), 9.2\left(\mathrm{CH}_{2} \mathrm{CH}_{3}\right)$. HRMS (ESI) $\mathrm{m} / z[\mathrm{M}+\mathrm{H}]^{+}$ calcd for $\left[\mathrm{C}_{21} \mathrm{H}_{26} \mathrm{~N}_{3} \mathrm{O}_{3} \mathrm{~S}\right]^{+} 400.1689$; found 400.1683. Anal. Calcd for $\mathrm{C}_{21} \mathrm{H}_{26} \mathrm{~N}_{3} \mathrm{O}_{3} \mathrm{~S} . \mathrm{HCl}$ : C 57.86, H 6.01, N 9.64, S 7.35. Found: C, 57.54;

\section{(Z)-2-Propionamido-5- $N, 6-S$-(N'-5-indolylmethyliminomethylidene)-1,2-dideoxy-6-}

thionojirimycin Hydrochloride (38). Column chromatography, eluent: 70:10:1 DCM-MeOH$\mathrm{H}_{2} \mathrm{O}$. Yield: $104 \mathrm{mg}$ (quantitative). $\mathrm{R}_{f}$ 0.40 (50:10:1 DCM-MeOH- $\mathrm{H}_{2} \mathrm{O}$ ).

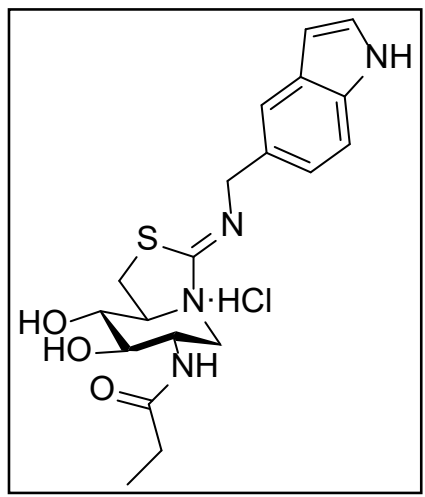

${ }^{1} \mathbf{H}$ NMR (500 MHz, $\left.\mathrm{CD}_{3} \mathrm{OD}\right): \delta 7.57-6.46\left(\mathrm{~m}, 5 \mathrm{H}, \mathrm{CH}_{\text {arom }}\right), 4.60$ (bs, $\left.2 \mathrm{H}, \mathrm{CH}_{2} \mathrm{Ph}\right), 4.18\left(\mathrm{dd}, 1 \mathrm{H}, J_{1 \mathrm{a}, 1 \mathrm{~b}}=13.3 \mathrm{~Hz}, J_{1 \mathrm{a}, 2}=5.3 \mathrm{~Hz}, \mathrm{H}-\right.$ 1a), 3.88 (m, $1 \mathrm{H}, \mathrm{H}-2), 3.83$ (m, $1 \mathrm{H}, \mathrm{H}-5), 3.68$ (m, $1 \mathrm{H}, J_{6 \mathrm{a}, 6 \mathrm{~b}}=$ $\left.11.3 \mathrm{~Hz}, J_{5,6 \mathrm{a}}=7.4 \mathrm{~Hz}, \mathrm{H}-6 \mathrm{a}\right), 3.52\left(\mathrm{t}, 1 \mathrm{H}, J_{2,3}=J_{3,4}=9.0, \mathrm{H}-3\right)$, 3.42 (m, 2 H, H-4, H-6b), 2.93 (dd, 1 H, $J_{1 b, 2}=11.5$ Hz, H-1b), 2.29 (q, $\left.2 \mathrm{H}, \mathrm{CH}_{2} \mathrm{CH}_{3}\right), 1.16$ (t, $\left.3 \mathrm{H}, \mathrm{CH}_{2} \mathrm{CH}_{3}\right) .{ }^{13} \mathbf{C} \mathbf{N M R}(125.7 \mathrm{MHz}$, $\left.\mathrm{CD}_{3} \mathrm{OD}\right): \delta 177.7(\mathrm{CO}), 169.9(\mathrm{CN}), 137.4-102.5\left(\mathrm{C}_{\text {arom }}\right), 75.9(\mathrm{C}-$

3), 75.1 (C-4), 68.4 (C-5), $55.8\left(\mathrm{CH}_{2} \mathrm{Ph}\right), 51.0(\mathrm{C}-2), 47.2$ (C-1), $32.2(\mathrm{C}-6), 30.3\left(\mathrm{CH}_{2} \mathrm{CH}_{3}\right), 10.4$ $\left(\mathrm{CH}_{2} \mathrm{CH}_{3}\right)$. HRMS (ESI) $m / z[\mathrm{M}+\mathrm{H}]^{+}$calcd for $\left[\mathrm{C}_{19} \mathrm{H}_{25} \mathrm{~N}_{4} \mathrm{O}_{3} \mathrm{~S}\right]^{+}$389.1642; found 389.1637. Anal. Calcd for $\mathrm{C}_{19} \mathrm{H}_{25} \mathrm{~N}_{4} \mathrm{O}_{3} \mathrm{~S} . \mathrm{HCl}$ : C 53.70, H 5.93, N 13.18, S 7.54. Found: C, 53.48; H, 5.70; N, 12.86; S, 7.21.

\section{Synthesis of DNJNAc-6S-NBn-Biotin probe}

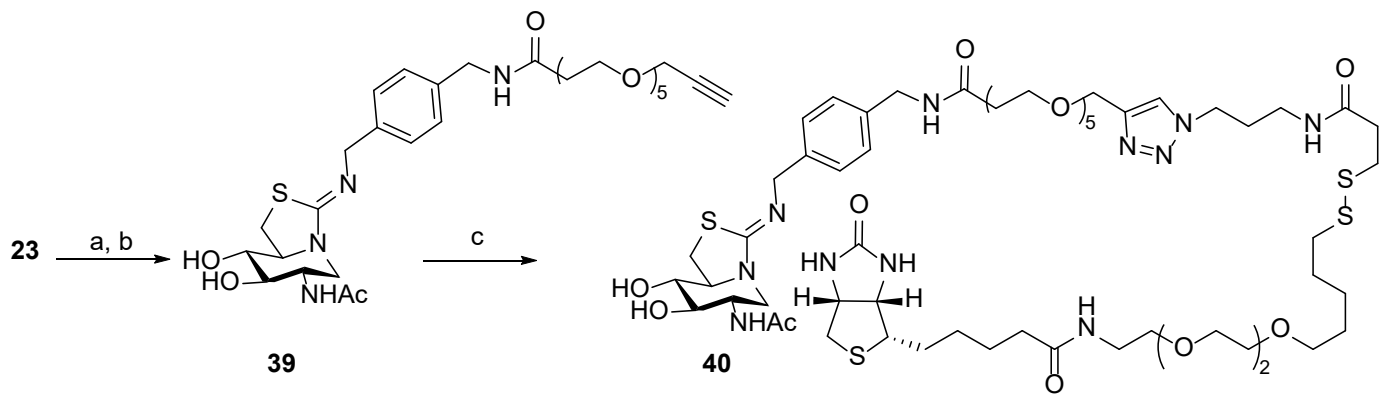

Supplemental scheme 8. Reagents and conditions: a. $\mathrm{PPh}_{3}, \mathrm{THF}-\mathrm{H}_{2} \mathrm{O}, \mathrm{RT}, 18 \mathrm{~h}$; b. alkyne-PEG4acid, HBTU, DIPEA, DMF, RT, 24 h; c. biotin-azide, sodium ascorbate, $\mathrm{CuSO}_{4} \cdot 5 \mathrm{H}_{2} \mathrm{O}$, DMF$\mathrm{H}_{2} \mathrm{O}, \mathrm{RT}, 18 \mathrm{~h}$. 
Alkyne intermediate (39). A mixture of inhibitor $23(13.5 \mathrm{mg}, 0.0316 \mathrm{mM})$ and $\mathrm{PPh}_{3}(9.1 \mathrm{mg}$, $0.0348 \mathrm{mM})$ in $4: 1$ THF- $\mathrm{H}_{2} \mathrm{O}(2.5 \mathrm{~mL})$ was stirred overnight and then concentrated with coevaporation with 1:1 toluene- $\mathrm{PrOH}$. The residue was dried under vacuum for $2.5 \mathrm{~h}$. To the residue was added HBTU (16.8 mg, $0.0442 \mathrm{mM})$ and alkyne-PEG4-acid (12.5 mg, $0.0411 \mathrm{mM})$ followed by anhydrous DMF $(1.5 \mathrm{~mL})$ and DIPEA $(16.6 \mu \mathrm{L}, 0.0948 \mathrm{mM})$. The reaction mixture was stirred overnight and then concentrated with co-evaporation with toluene. The resulting residue was purified by CombiFlash (dry load, $4 \mathrm{~g}$ RediSep Gold Column, $18 \mathrm{~mL} / \mathrm{min}$ ) using a gradient of 1:0:0 $\rightarrow$ 6:3:1 EtOAc-MeOH- $\mathrm{H}_{2} \mathrm{O}$ to give intermediate $39(12.9 \mathrm{mg}, 64 \%)$ as a clear viscous oil. $\mathrm{R}_{f} 0.41\left(9: 3: 1\right.$ EtOAc-MeOH- $\left.\mathrm{H}_{2} \mathrm{O}\right)$.

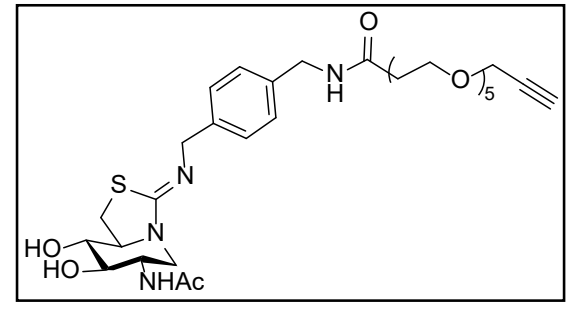

${ }^{1} \mathbf{H}$ NMR (600 MHz, $\left.\mathrm{CD}_{3} \mathrm{OD}\right): \delta 7.27(\mathrm{~d}, 2 \mathrm{H}, J=8.3 \mathrm{~Hz}$, $\mathrm{CH}$-arom), 7.25 (d, $\left.2 \mathrm{H}, J=8.6 \mathrm{~Hz}, \mathrm{CH}_{\text {arom }}\right), 4.39$ (d, $1 \mathrm{H}$, $\left.J=14.8 \mathrm{~Hz}, \mathrm{PhCH}_{2} \mathrm{~N}\right), 4.38\left(\mathrm{~s}, 2 \mathrm{H}, \mathrm{PhCH}_{2} \mathrm{~N}\right), 4.33(\mathrm{~d}, 1$ $\left.\mathrm{H}, J=14.9 \mathrm{~Hz}, \mathrm{ArCH}_{2} \mathrm{~N}\right), 4.19\left(\mathrm{~s}, 2 \mathrm{H}, \mathrm{OCH}_{2} \mathrm{C}\right), 4.16(\mathrm{dd}$, $1 \mathrm{H}, J=12.7,5.2 \mathrm{~Hz}, \mathrm{H}-1$ ), 3.86 (ddd, $1 \mathrm{H}, J=11.2,10.2$, $5.2 \mathrm{~Hz}, \mathrm{H}-2), 3.77$ (t, $2 \mathrm{H}, J=6.0 \mathrm{~Hz}, \mathrm{COCH}_{2} \mathrm{CH}_{2} \mathrm{O}$ ), 3.71-3.62 (m, $\left.16 \mathrm{H}, 8 \times \mathrm{OCH}_{2}\right), 3.47$ (dd, $1 \mathrm{H}, J=10.9,5.9 \mathrm{~Hz}, \mathrm{H}-6), 3.40$ (dd, $1 \mathrm{H}, J=10.2,8.4 \mathrm{~Hz}, \mathrm{H}-3), 3.36-3.32$ (m, 2 H, H-4, H-5), 3.18 (dd, $1 \mathrm{H}, J=10.8,7.1 \mathrm{~Hz}, \mathrm{H}-6$ '), 2.89 (t, $1 \mathrm{H}, J=2.4 \mathrm{~Hz}, \mathrm{C}_{\mathrm{q}} \mathrm{CH}$ ), 2.59 (dd, $1 \mathrm{H}, J=12.8$, $11.3 \mathrm{~Hz}, \mathrm{H}-1$ '), 2.51 (t, $2 \mathrm{H}, J=6.0 \mathrm{~Hz}, \mathrm{COCH}_{2} \mathrm{CH}_{2} \mathrm{O}$ ), 1.99 (s, $\left.3 \mathrm{H}, \mathrm{COCH}_{3}\right) .{ }^{13} \mathbf{C}$ NMR (150 $\left.\mathrm{MHz}, \mathrm{CD}_{3} \mathrm{OD}\right): \delta 172.5,172.2(\mathrm{CO}), 161.8(\mathrm{CN}), 139.2,136.9,127.2,127.1\left(\mathrm{C}_{\text {arom }}\right), 79.2$ $\left(\mathrm{C}_{\mathrm{q}} \mathrm{CH}\right), 75.6(\mathrm{C} 3), 74.1(\mathrm{C} 4), 70.1\left(\mathrm{OCH}_{2}\right), 70.0\left(\mathrm{OCH}_{2}\right), 69.9\left(\mathrm{OCH}_{2}\right), 68.7\left(\mathrm{OCH}_{2}\right), 66.9$ $\left(\mathrm{COCH}_{2} \mathrm{CH}_{2} \mathrm{O}\right), 64.6$ (C5), $57.6\left(\mathrm{OCH}_{2} \mathrm{C}_{\mathrm{q}}, \mathrm{NCH}_{2} \mathrm{Ar}\right), 57.5\left(\mathrm{NCH}_{2} \mathrm{Ar}\right), 49.9(\mathrm{C} 2), 45.8(\mathrm{C} 1), 36.3$ $\left(\mathrm{COCH}_{2} \mathrm{CH}_{2}\right), 29.9(\mathrm{C} 6), 21.4\left(\mathrm{COCH}_{3}\right)$. HRMS (ESI) $\mathrm{m} / z[\mathrm{M}+\mathrm{H}]^{+}$calcd for $\mathrm{C}_{31} \mathrm{H}_{47} \mathrm{~N}_{4} \mathrm{O}_{9} \mathrm{~S}$ : 651.3064 , found 651.3631 .

DNJNAc-6S-NBn-Biotin (40). To a mixture of intermediate $39(4.7 \mathrm{mg}, 0.0072 \mathrm{mmol})$ and biotin-SS-azide (5.5 mg, $0.0079 \mathrm{mM}$ ) obtained from ClickChemistryTools (1168-25,) in DMF $(250 \mu \mathrm{L})$ was added a solution of sodium ascorbate $(1.4 \mathrm{mg}, 0.00722 \mathrm{mM})$ and $\mathrm{CuSO}_{4} \cdot 5 \mathrm{H}_{2} \mathrm{O}(0.9$ $\mathrm{mg}, 0.00361 \mathrm{mM})$ in $\mathrm{H}_{2} \mathrm{O}(250 \mu \mathrm{L})$. The reaction mixture was stirred overnight at RT. The mixture was concentrated and co-evaporated with a 1:1 toluene- $\mathrm{PrOH}$ solution, then dried under vacuum for $1 \mathrm{~h}$. The resulting residue was purified by HPLC (XDB C18 semi-prep column) to

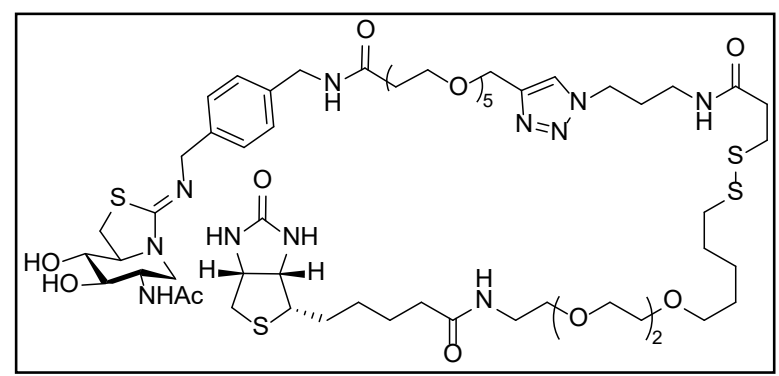
give the final DNJNAc-6S-NBn-Biotin (40) $(3.0 \mathrm{mg}, 31 \%)$ as a colorless oil. $\mathrm{R}_{f} 0.20(4: 3: 1$ EtOAc-MeOH- $\mathrm{H}_{2} \mathrm{O}$ ).

${ }^{1} \mathbf{H}$ NMR (600 MHz, $\left.\mathrm{CD}_{3} \mathrm{OD}\right): \delta 8.05(\mathrm{~s}, 1 \mathrm{H}$, $\mathrm{CH}_{\text {arom }}$ ), 7.39-7.32 (m, $\left.4 \mathrm{H}, \mathrm{CH}_{\text {arom }}\right), 4.64$ (s, 
$\left.2 \mathrm{H}, \mathrm{OCH}_{2} \mathrm{Ph}\right), 4.60$ (s, $\left.2 \mathrm{H}, \mathrm{NCH}_{2} \mathrm{Ph}\right), 4.52\left(\mathrm{dd}, 1 \mathrm{H}, J=13.3,5.1 \mathrm{~Hz}, \mathrm{C} H \mathrm{~N}_{\text {biotin }}\right), 4.47$ (t, $2 \mathrm{H}, J$ $\left.=6.8 \mathrm{~Hz}, \mathrm{~N}_{\mathrm{Ar}} \mathrm{CH}_{2} \mathrm{CH}_{2} \mathrm{CH}_{2}\right), 4.42\left(\mathrm{~s}, 2 \mathrm{H}, \mathrm{ArCH}_{2} \mathrm{~N}\right), 4.33\left(\mathrm{dd}, 1 \mathrm{H}, J=7.9,4.4 \mathrm{~Hz}, \mathrm{C} H \mathrm{~N}_{\text {biotin }}\right), 4.18$ (dd, $1 \mathrm{H}, J=12.4,5.2 \mathrm{~Hz}, \mathrm{H}-1$ ), 4.09 (app. q, $1 \mathrm{H}, J=8.3 \mathrm{~Hz}, \mathrm{H}-5$ ), 3.86 (ddd, $1 \mathrm{H}, J=11.9$, 10.7, $5.1 \mathrm{~Hz}, \mathrm{H}-2), 3.81-3.75$ (m, $\left.3 \mathrm{H}, \mathrm{H}-6, \mathrm{COCH}_{2} \mathrm{CH}_{2} \mathrm{O}_{\mathrm{L} 1}\right), 3.73-3.45$ (m, $28 \mathrm{H}, \mathrm{H}-3, \mathrm{H}-4$, $\mathrm{COCH}_{2} \mathrm{CH}_{2} \mathrm{O}_{\mathrm{L} 2}, 13 \times \mathrm{OCH}_{2}$ ), 3.42-3.37 (m, 4H, $\mathrm{OCH}_{2} \mathrm{CH}_{2} \mathrm{~N}_{\mathrm{L} 2}, \mathrm{SCH}_{2} \mathrm{CH}_{2} \mathrm{~N}_{\mathrm{L} 2}$ ), 3.26-3.21 (m, 3 $\left.\mathrm{H}, \mathrm{CHS}_{\text {biotin, }} \mathrm{CH}_{2} \mathrm{CH}_{2} \mathrm{CH}_{2} \mathrm{~N}_{(\mathrm{L} 2)}\right), 3.08$ (dd, $1 \mathrm{H}, J=12.2,12.2 \mathrm{~Hz}, \mathrm{H}-1$ '), $3.03-2.91$ (m, $5 \mathrm{H}$, $\left.\mathrm{CH}_{2} \mathrm{~S}_{\text {biotin, }} \mathrm{CH}_{2} \mathrm{CH}_{2} \mathrm{~S}_{\mathrm{L} 2}, \mathrm{SCH}_{2} \mathrm{CH}_{2(\mathrm{~L} 2)}\right), 2.73$ (d, $\left.1 \mathrm{H}, J=12.7 \mathrm{~Hz}, \mathrm{CH}_{2}{ }^{\prime} \mathrm{S}_{\text {biotin }}\right), 2.66-2.61$ (m, $4 \mathrm{H}$, $\mathrm{COCH}_{2} \mathrm{CH}_{2} \mathrm{~S}_{\mathrm{L} 2}, \mathrm{COCH}_{2} \mathrm{CH}_{2} \mathrm{O}_{\mathrm{L} 2}$ ), 2.52 (t, $2 \mathrm{H}, J=5.8 \mathrm{~Hz}, \mathrm{COCH}_{2} \mathrm{CH}_{2} \mathrm{O}_{\mathrm{L} 1}$ ), 2.24 (t, $2 \mathrm{H}, J=7.3$

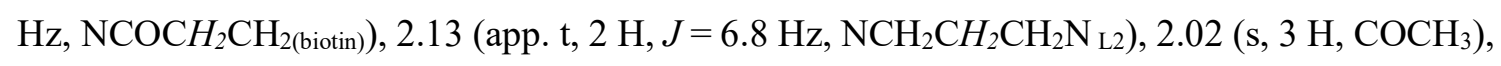

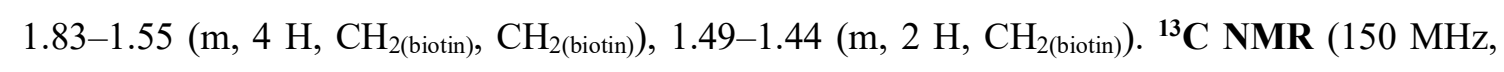
$\left.\mathrm{CD}_{3} \mathrm{OD}\right): \delta 174.7,172.7,172.5,172.3,172.0,139.4,133.5,127.7,127.6,73.8,73.2,70.2,70.1$ (4×), $70.0(2 \times), 69.8(3 \times), 69.4,69.2,69.1,68.1,66.9,62.0,60.2,55.6,51.1,49.5,45.6,42.2$, 39.7, 39.1, 39.0, 36.4, 36.1, 35.4, 35.1, $35.0(2 \times)$, 33.7, 33.6, 31.1, 29.7, 28.4, 28.1, 25.5, 21.3. HRMS (ESI) $m / z$ [M+NH$]^{+}$calcd for $\mathrm{C}_{59} \mathrm{H}_{101} \mathrm{~N}_{12} \mathrm{O}_{15} \mathrm{~S}_{4}: 1345.5828$, found 1345.5766 . 


\section{Morrison $K_{\mathrm{i}}$ determination against human OGA, HexA and HexB}

13

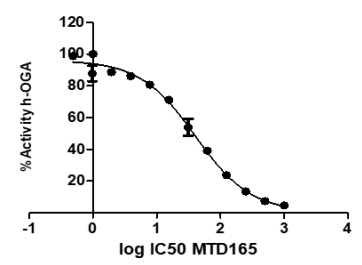

14

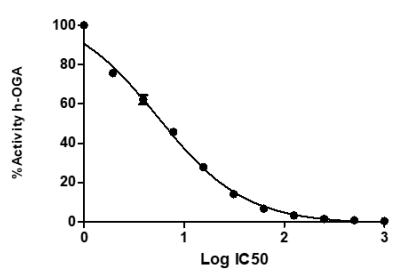

16

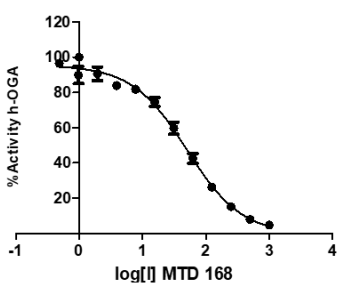

Supplemental Figure 1. $K_{\mathrm{i}}$ determination of $\mathbf{1 3}(24 \pm 8 \mathrm{nM}), \mathbf{1 4}(20 \pm 7 \mathrm{nM})$ and $\mathbf{1 6}(3 \pm 1 \mathrm{nM})$ against hOGA.

18

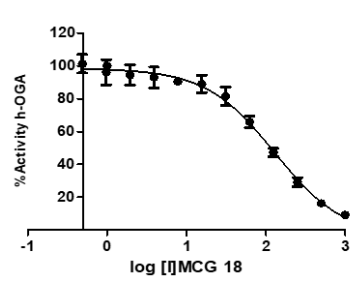

19

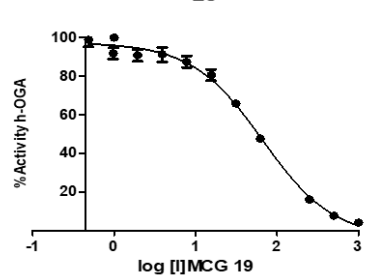

20

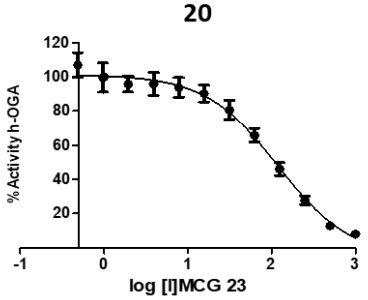

Supplemental Figure 2. $K_{\mathrm{i}}$ determination of $18(50 \pm 20 \mathrm{nM}), \mathbf{1 9}(30 \pm 10 \mathrm{nM})$, and $20(50 \pm$ $20 \mathrm{nM}$ ), against hOGA.
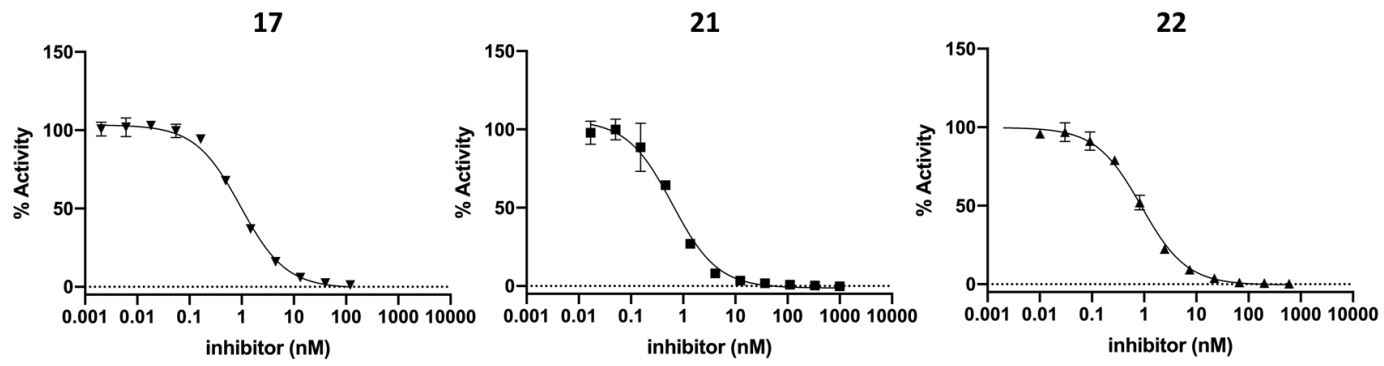

Supplemental Figure 3. $K_{\mathrm{i}}$ determination of $\mathbf{1 7}(0.3 \pm 0.1 \mathrm{nM}), \mathbf{2 1}(0.27 \pm 0.09 . \mathrm{nM})$, and 22 $(0.22 \pm 0.07 \mathrm{nM})$, against hOGA. 

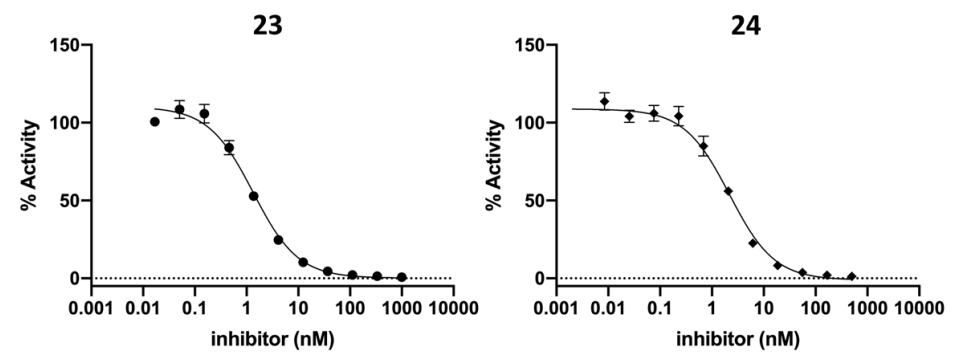

Supplemental Figure 4. $K_{\mathrm{i}}$ determination of $23(0.4 \pm 0.2 \mathrm{nM})$, and $24(0.9 \pm 0.3 \mathrm{nM})$, against hOGA.
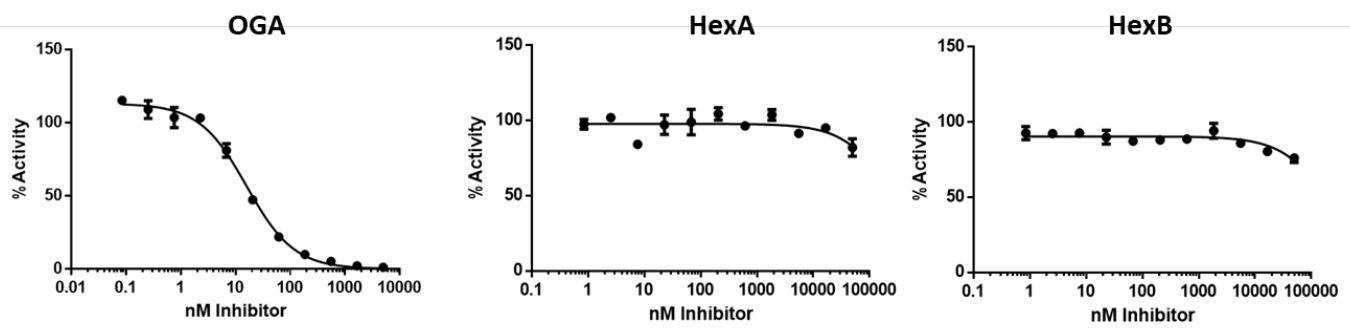

Supplemental Figure 5. $K_{\mathrm{i}}$ determination of 28 against hOGA $(8 \pm 3 \mathrm{nM}), \operatorname{HexA}(<100 \mu \mathrm{M})$ and $\operatorname{HexB}(<100 \mu \mathrm{M})$.
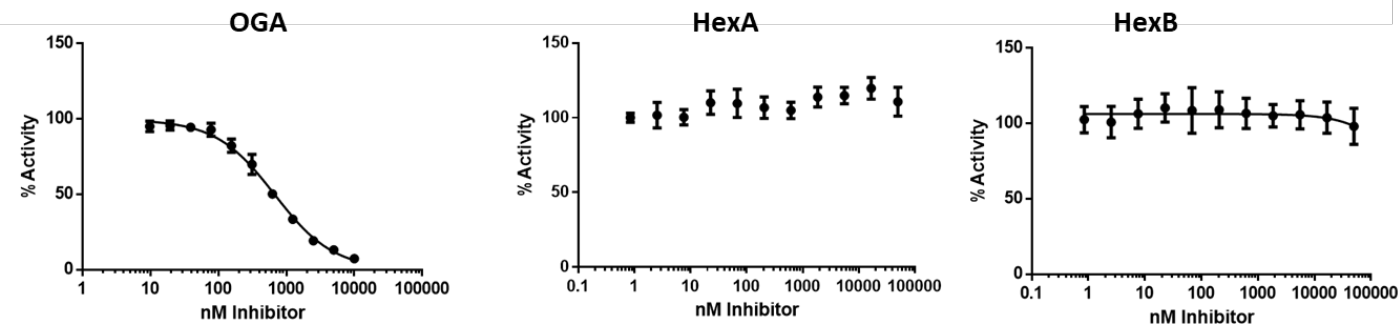

Supplemental Figure 6. $K_{\mathrm{i}}$ determination of $29(900 \pm 300 \mathrm{nM})$ against hOGA, HexA $(<100 \mu \mathrm{M})$ and $\operatorname{HexB}(<100 \mu \mathrm{M})$.
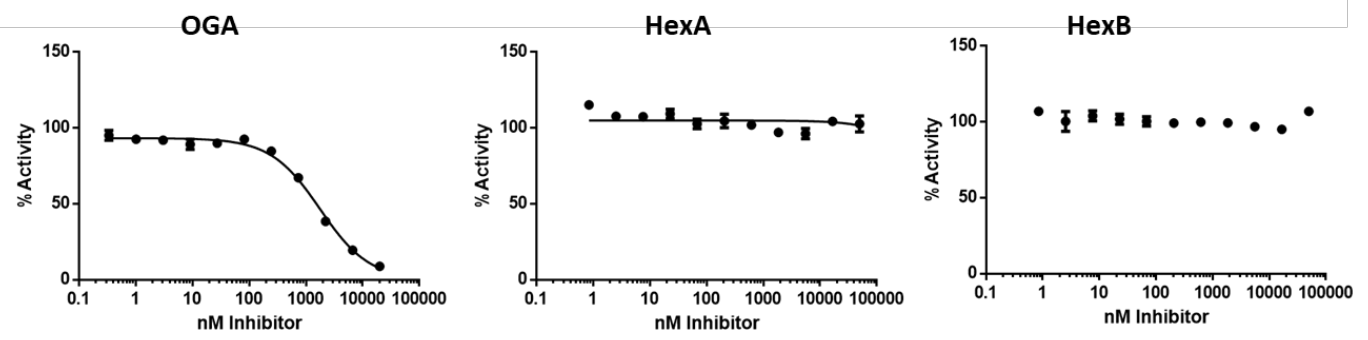

Supplemental Figure 7. $K_{\mathrm{i}}$ determination of $\mathbf{3 0}(300 \pm 100 \mathrm{nM})$ against hOGA, HexA $(<100 \mu \mathrm{M})$ and $\operatorname{HexB}(<100 \mu \mathrm{M})$. 

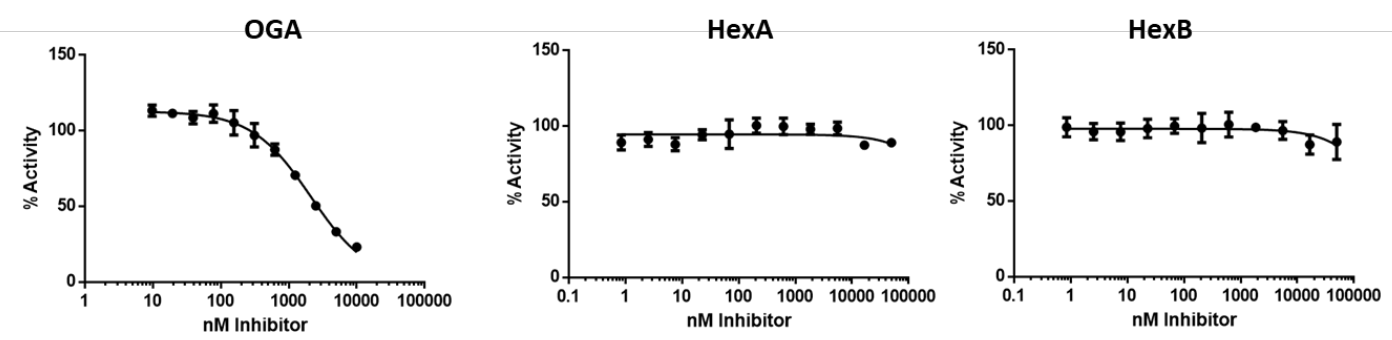

Supplemental Figure 8. $K_{\mathrm{i}}$ determination of $\mathbf{3 1}$ against hOGA $(1000 \pm 400 \mathrm{nM})$, HexA $(<100$ $\mu \mathrm{M})$ and $\operatorname{HexB}(<100 \mu \mathrm{M})$.
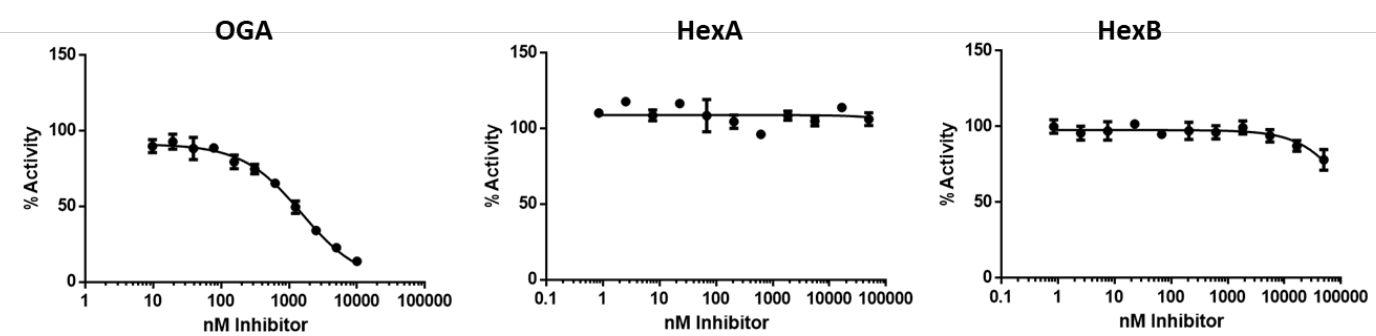

Supplemental Figure 9. $K_{\mathrm{i}}$ determination of $\mathbf{3 2}$ against hOGA $(800 \pm 300 \mathrm{nM})$, HexA $(<100$ $\mu \mathrm{M})$ and $\operatorname{HexB}(<100 \mu \mathrm{M})$.
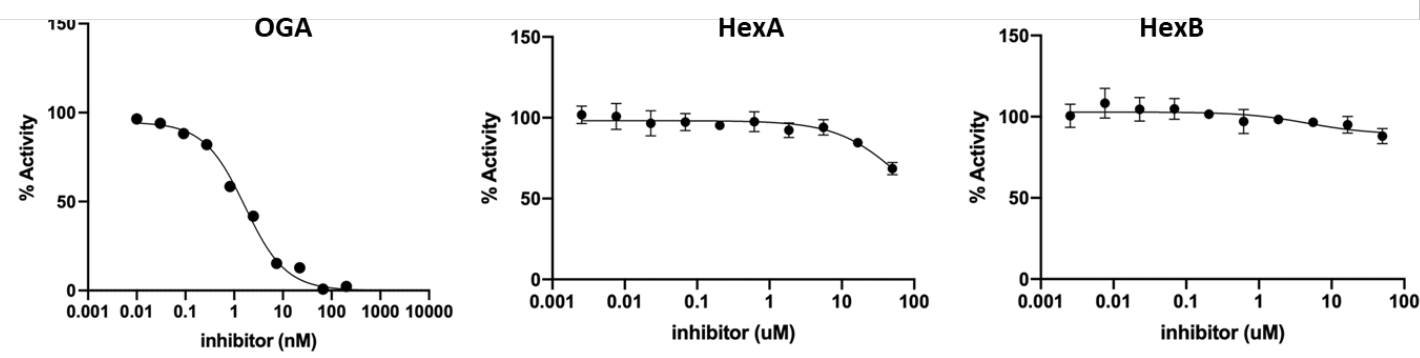

Supplemental Figure 10. $K_{\mathrm{i}}$ determination of 37 against hOGA $(0.8 \pm 0.2 \mathrm{nM})$, HexA $(<100$ $\mu \mathrm{M})$ and $\operatorname{HexB}(<100 \mu \mathrm{M})$.
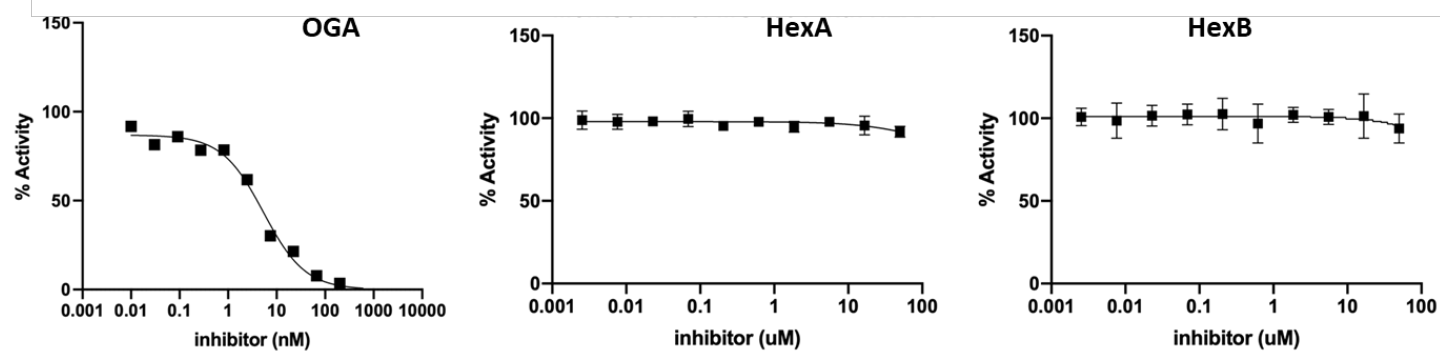
Supplemental Figure 11. $K_{\mathrm{i}}$ determination of 38 against hOGA $(2.4 \pm 0.9 \mathrm{nM})$, HexA $(<100$ $\mu \mathrm{M})$ and $\operatorname{HexB}(<100 \mu \mathrm{M})$.
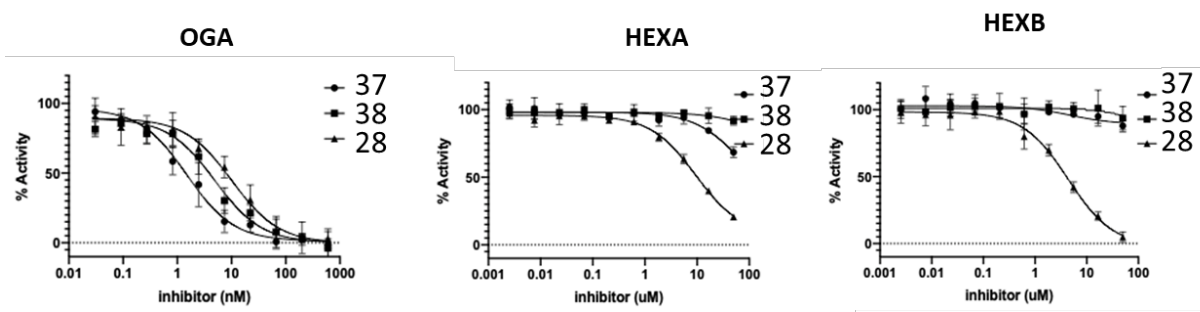

Supplemental Figure 12. Comparative $K_{\mathrm{i}}$ determination of 28, 37 and 38 against hOGA, HexA $(<100 \mu \mathrm{M})$ and $\operatorname{HexB}(<100 \mu \mathrm{M})$.

Inhibition of compounds 1-24 against commercial hexosaminidases.

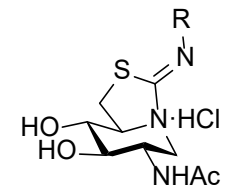

Supplemental Table 1. Inhibition constants (Ki) for DNJNAc-thiazolidines 13-24 against commercial hexosaminidases

\begin{tabular}{|c|c|c|c|}
\hline & & Bovine kidney Hex & Human placenta Hex \\
\hline Compound & Structure (R) & $K_{\mathrm{i}}(\mu \mathrm{M})$ & $K_{\mathrm{i}}(\mu \mathrm{M})$ \\
\hline 13 & $(\mathrm{CH} 2)_{3} \mathrm{CH}_{3}$ & $0.57 \pm 0.04$ & $0.24 \pm 0.02$ \\
\hline 14 & $(\mathrm{CH} 2)_{7} \mathrm{CH}_{3}$ & $0.19 \pm 0.02$ & $0.24 \pm 0.02$ \\
\hline 15 & $\mathrm{Ph}$ & $0.70 \pm 0.05$ & $0.58 \pm 0.04$ \\
\hline 16 & $\mathrm{CH}_{2} \mathrm{Ph}$ & $0.46 \pm 0.03$ & $0.42 \pm 0.03$ \\
\hline 17 & $\mathrm{CH}_{2}$-1-naphthyl & $0.89 \pm 0.8$ & n.d. ${ }^{\mathrm{a}}$ \\
\hline 18 & $\mathrm{CH}_{2} \mathrm{p}-\mathrm{FPh}$ & $1.5 \pm 0.2$ & $0.28 \pm 0.02$ \\
\hline 19 & $\mathrm{CH}_{2} \mathrm{p}-\mathrm{OMePh}$ & $1.2 \pm 0.1$ & $0.73 \pm 0.04$ \\
\hline 20 & $\mathrm{CH}_{2} \mathrm{p}-\mathrm{CF}_{3} \mathrm{Ph}$ & $0.43 \pm 0.04$ & $0.40 \pm 0.03$ \\
\hline 21 & $\mathrm{CH}_{2} \mathrm{O}-\mathrm{CH}_{2} \mathrm{~N}_{3} \mathrm{Ph}$ & $0.91 \pm 0.09$ & n.d. ${ }^{\mathrm{a}}$ \\
\hline 22 & $\mathrm{CH}_{2} \mathrm{~m}-\mathrm{CH}_{2} \mathrm{~N}_{3} \mathrm{Ph}$ & $0.83 \pm 0.06$ & n.d. ${ }^{\mathrm{a}}$ \\
\hline 23 & $\mathrm{CH}_{2} \mathrm{p}-\mathrm{CH}_{2} \mathrm{~N}_{3} \mathrm{Ph}$ & $0.72 \pm 0.05$ & n.d. ${ }^{\mathrm{a}}$ \\
\hline 24 & $\mathrm{CH}_{2} \mathrm{CH}_{2} \mathrm{Ph}$ & $1.4 \pm 0.12$ & n.d. ${ }^{\mathrm{a}}$ \\
\hline
\end{tabular}




\section{X-Ray structure solution methods for hOGA and BtGH84 in complex with compound 16}

hOGA was produced and purified as a split construct, DNA encoding residues 11-396 and 535715 was inserted in two separate plasmids, according to the protocol published by Roth, et al. $2017^{6}$. hOGA was crystallised by hanging drop vapour diffusion method at a concentration of 30 $\mathrm{mg} \mathrm{mL}-1$ under the conditions of $200 \mathrm{mM}$ Triammonium citrate $\mathrm{pH} 7.5$ and $24 \%$ Polyethylene glycol 3350. A seed stock was added at $20 \mathrm{x}$ diluted concentration in a $1: 0.2: 0.8 \mu \mathrm{L}$ ratio of protein : seed stock : reservoir. BtGH84 was produced and purified as described previously by Dennis et al, 2006 ${ }^{7}$. Bt GH84 was crystallised by sitting drop vapour diffusion method at a concentration of $12 \mathrm{mg} \mathrm{mL}^{-1}$ under the conditions of $125 \mathrm{mM}$ imidazole $\mathrm{pH} 8,8 \%$ PEG 8000, 3\% trimethylamine N-oxide dehydrate, $15 \%$ ethylene glycol in a 1:1 ratio of protein to reservoir solution. Crystals were soaked with compound $\mathbf{1 6}$ at a final concentration of $5 \mathrm{mM}$ for either 2 hours or 7 days, $B t$ GH84 and hOGA respectively. Diffraction data were collected on the I03 and I04-1 beamlines at Diamond Light Source (DLS). The data were indexed, integrated and scaled using $\mathrm{Xia} 2^{8}$ and reintegrated using Aimless ${ }^{9,10}$ from the CCP4 software ${ }^{11}$; the data collection statistics are shown in table 2. Molecular replacement was conducted using Molrep ${ }^{12}$ with coordinates $5 \mathrm{M} 7 \mathrm{R}$ and $5 \mathrm{ABE}$ for hOGA and $\mathrm{Bt} \mathrm{GH} 84$, respectively. The model was adjusted and refined using alternating cycles of manual model building and real space refinement in $\operatorname{Coot}^{13}$ and reciprocal space refinement in Refmac ${ }^{14-17}$. Data quality was assessed by the multimeric model geometry validation programme ${ }^{18}$ in CCP4 and Privateer ${ }^{19}$ was used to assess the confidence of the carbohydrate ligand conformation. Figures were produced using $\mathrm{CCP} 4 \mathrm{mg}^{20}$. 
Supplemental Table 2. Data collection and refinement statistics for hOGA and BtGH84 enzymes in complex with compound $\mathbf{1 6}$.

\begin{tabular}{|c|c|c|}
\hline & $h$ OGA_16 & $B t \mathrm{GH} 84 \_16$ \\
\hline PDB code & $70 U 6$ & $70 U 8$ \\
\hline \multicolumn{3}{|l|}{ Data collection } \\
\hline Beamline & Diamond I03 & Diamond I04-1 \\
\hline Wavelength $(\AA)$ & 0.9763 & 0.9159 \\
\hline Space group & $P 4_{3} 2_{1} 2$ & $P 222_{1}$ \\
\hline $\begin{array}{l}\text { Cell dimensions: } \\
a, b, c(\AA)\end{array}$ & $101.29,101.29,284.52$ & $51.53,160.62,224.51$ \\
\hline$\alpha, \beta, \gamma\left({ }^{\circ}\right)$ & $90.00,90.00,90.00$ & $90.00,90.00,90.00$ \\
\hline Resolution $(\AA)$ & $58.21-2.41(2.48-2.41)$ & $75.62-1.50(1.53-1.50)$ \\
\hline$R_{\text {merge }}$ & $0.05(2.28)$ & $0.07(0.81)$ \\
\hline$R_{\text {pim }}$ & $0.01(0.59)$ & $0.03(0.37)$ \\
\hline$C C(1 / 2)$ & $1.00(0.56)$ & $1.00(0.65)$ \\
\hline$I / \sigma I$ & $26.5(1.5)$ & $12.3(1.5)$ \\
\hline Completeness (\%) & $100.0(100.0)$ & $100.0(100.0)$ \\
\hline Redundancy & $15.8(15.6)$ & $7.5(5.8)$ \\
\hline \multicolumn{3}{|l|}{ Refinement } \\
\hline Resolution $(\AA)$ & $58.21-2.41(2.48-2.41)$ & $75.62-1.50(1.53-1.50)$ \\
\hline No. reflections & 58168 & 298007 \\
\hline$R_{\text {work }} / R_{\text {free }}$ & $0.22 / 0.27$ & $0.21 / 0.23$ \\
\hline \multicolumn{3}{|l|}{ No. atoms } \\
\hline Protein & 6704 & 11529 \\
\hline Ligands/Ions & 46 & $50 / 1$ \\
\hline Water & 18 & 903 \\
\hline \multicolumn{3}{|l|}{$B$-factors $\left(\AA^{2}\right)$} \\
\hline Protein & 89 & 28 \\
\hline Ligand/Ions & 82 & $19 / 23$ \\
\hline Water & 65 & 31 \\
\hline
\end{tabular}




$\begin{array}{lll}\text { Bond lengths }(\AA) & 0.007 & 0.011 \\ \text { Bond angles }\left(^{\circ}\right) & 1.52 & 1.74\end{array}$

Ramachandran

Favoured (\%) $\quad 94.2 \quad 95.8$

Allowed (\%) $\quad 4.7 \quad 3.6$

Outliers $(\%) \quad 1.1 \quad 0.6$
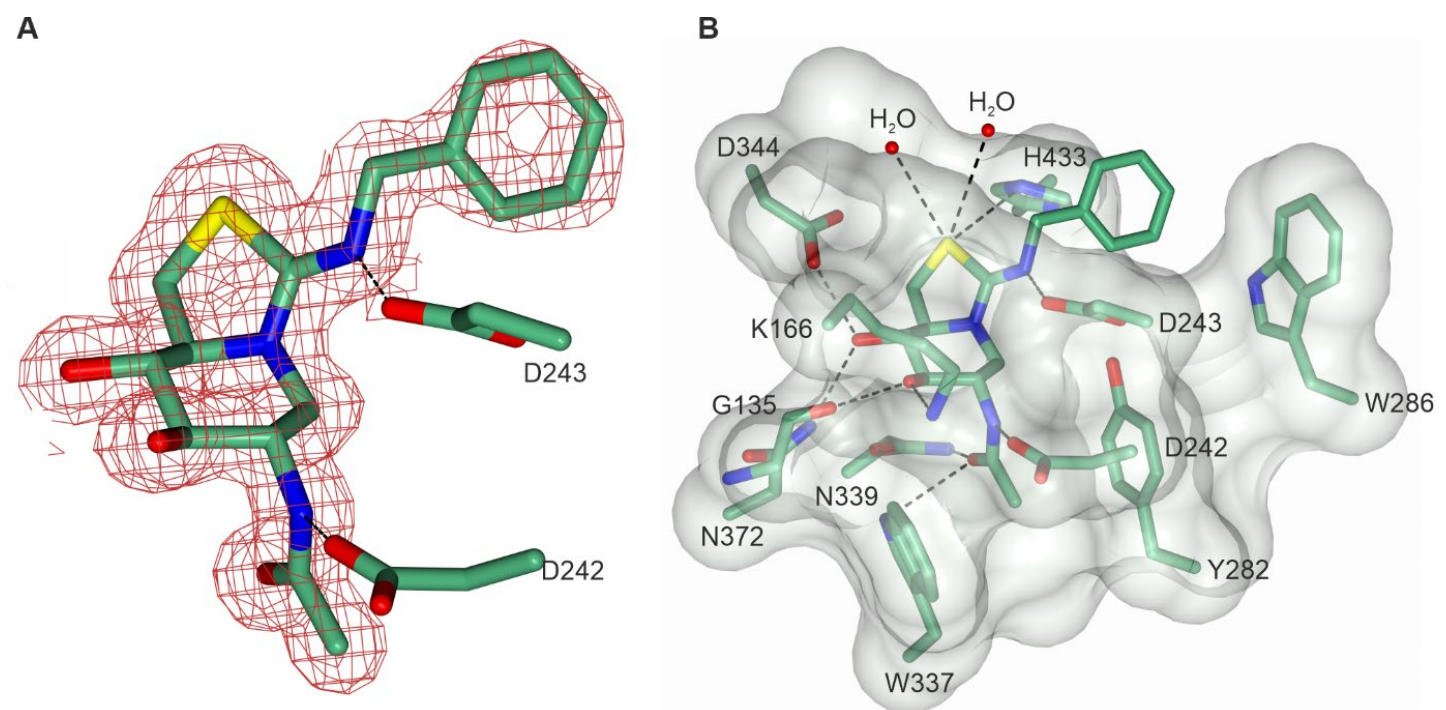

Supplemental Figure 13. Structure of $B t \mathrm{GH} 84$ in complex with compound 16. Active site residues of $B t \mathrm{GH} 84$ are shown in green. (A) $\mathbf{1 6}$ is shown in dark green with the corresponding REFMAC maximum-likelihood/ $\sigma$ A-weighted $2 F_{0}-F_{\mathrm{c}}$ map, shown in red, contoured at 0.46 electrons $\AA^{-3}$. (B) Surface representation of the active site pocket of $B t \mathrm{GH} 84$ with neighbouring residues, waters and hydrogen bond interactions highlighted. 


\section{Chemoproteomic and mapping of post-transcriptional modifications}

Preliminary pull-down experiments with recombinant hOGA. Recombinant hOGA was added to lysis buffer (25 mM HEPES, $150 \mathrm{mM} \mathrm{NaCl}, 5 \mathrm{mM}$ EDTA, 5\% glycerol, pH 7.1) containing protease inhibitor cocktail (Roche cOmplete ${ }^{\mathrm{TM}}$, EDTA free) at a concentration of 100 $\mathrm{nM}$ at $4^{\circ} \mathrm{C}$. DNJNAc-6S-NBn-Biotin was added at concentration of $500 \mathrm{nM}$ and incubated with rotation for $1 \mathrm{~h}$ at $4{ }^{\circ} \mathrm{C}$. The sample was then incubated with the streptavidin beads (Tri-Link Biotechnologies) for $1 \mathrm{~h}$ at $4{ }^{\circ} \mathrm{C}$. The beads were separated and washed with lysis buffer 5 times at $4{ }^{\circ} \mathrm{C}$. The beads were then eluted with $100 \mathrm{mM}$ DTT in lysis buffer.

Large scale precipitation of bovine OGA. Bovine brains were obtained from Animal Technologies, Inc. The lysate was prepared using a 4:1 lysis buffer: dry cell extractions. The entire brain was homogenized by cryomilling under liquid nitrogen. The brain tissue was processed using a dounce homogenizer in 4:1 lysis buffer (25 mM HEPES, $133 \mathrm{mM} \mathrm{KCl}, 5 \mathrm{mM}$ $\mathrm{MgCl} 2,5$ mM EDTA, 10\% Glycerol, pH 7.1, SIGMAFAST ${ }^{\mathrm{TM}}$ Protease Inhibitor Cocktail) to dry tissue, at $4{ }^{\circ} \mathrm{C}$. The lysate was cleared by centrifugation (Sorvall RC-6 Plus) at 20,000 RPM for 30 min at $4{ }^{\circ} \mathrm{C}$. The supernatant was collected and the remaining debris was removed by ultracentrifugation (Beckman Optima Ultracentrifuge L-80 XP) at 45,000 RPM for 45 min at $4{ }^{\circ} \mathrm{C}$. The supernatant was collected and separated into fresh tubes. The samples were incubated for 30 min at $4{ }^{\circ} \mathrm{C}$ with streptavidin beads to deplete endogenously biotinylated proteins. The sample was centrifuged and the supernatant collected to remove the beads from the sample after which $128 \mathrm{nM}$ of DNJNAc-6S-NBn-Biotin was added and the sample was mixed by rotation for $1 \mathrm{hr}$ at $4{ }^{\circ} \mathrm{C}$. A parallel control experiment was performed in which we added a high concentration of Thiamet-G $(1 \mathrm{mM})$ to block binding to the DNJNAc-6S-NBn-Biotin ligand. The sample and control were then incubated with the beads $(200 \mu \mathrm{L})$ for $1 \mathrm{hr}$ at $4{ }^{\circ} \mathrm{C}$. The beads were separated and washed with cold lysis buffer 5 times at $4{ }^{\circ} \mathrm{C}$. The affinity purified protein and control were then eluted by addition of DTT in lysis buffer to provide a final concentration of $60 \mathrm{mM}$ and the samples were mixed using a thermomixer (Eppendorf) at $37^{\circ} \mathrm{C}$ for $10 \mathrm{~min}$.

LC-MS/MS sample preparation and analysis. The samples were loaded onto $12 \%$ SDS-PAGE minigels (Bio-Rad), electrophoresed, and stained with Coomassie blue G-250 (Thermo Fisher Scientific). The band corresponding to OGA at the $130 \mathrm{kDa}$ range was excised, destained, protein disulfide bonds were reduced (10 $\mathrm{mM}$ DTT in $\left.50 \mathrm{mM} \mathrm{NH}_{4} \mathrm{HCO}_{3}, 45 \mathrm{~min}, 56{ }^{\circ} \mathrm{C}\right)$, after which thiols were alkylated $\left(55 \mathrm{mM}\right.$ iodoacetamide in $50 \mathrm{mM} \mathrm{NH} \mathrm{NHCO}_{3}$, dark, $30 \mathrm{~min}$, room temperature). Protein was subsequently digested (trypsin, Promega, in $50 \mathrm{mM} \mathrm{NH}_{4} \mathrm{HCO}_{3}$, overnight, $37^{\circ} \mathrm{C}$ ) and the resulting peptides were extracted with a gradient of acetonitrile solutions starting with aqueous $0.1 \%$ formic acid. Peptide mixtures were purified by solid-phase extraction 
using C18 StageTips (Thermo Fisher Scientific). $200 \mathrm{ng}$ of sample was injected into LC-MS/MS, analysis was performed using a Bruker timfTOF coupled to a Bruker nanoElute UHPLC system (Thermo Fisher Scientific).

Acquired data was then searched against the Uniprot protein database for Bos taurus using the Byonic $^{\mathrm{TM}}$ (v4.0.12 - ProteinMetrics Inc.) search algorithm from Protein Metrics Inc, with $15 \mathrm{ppm}$ and $40 \mathrm{ppm}$ mass accuracies for precursor and product ion masses respectively, and a $1 \%$ false discovery rate cut-off. Assigning of peptide and protein identifications included carbamidomethyl @ C (fixed), oxidation@M (common1) and deamidation@ N,Q (common1)modifications, RK fully specific digestion (maximum two missed cleavages), 15 and $40 \mathrm{ppm}$ precursor and fragment mass tolerance. Several variable modifications were selected for a second pass search, these can be found in Supplemental Spreadsheet. Focusing on the enzymatic modifications, an FDR criterion was defined for the modifications identified on a peptide hits as those with a $\mid \log$ Prob $\mid$ $>1.52(3 \% \mathrm{FDR})$, and high-confidence modifications were assigned as $\mid \log$ Prob $\mid>2.0(1 \%$ FDR). (Additional information regarding search parameters selected can be found in Supplemental Spreadsheet)

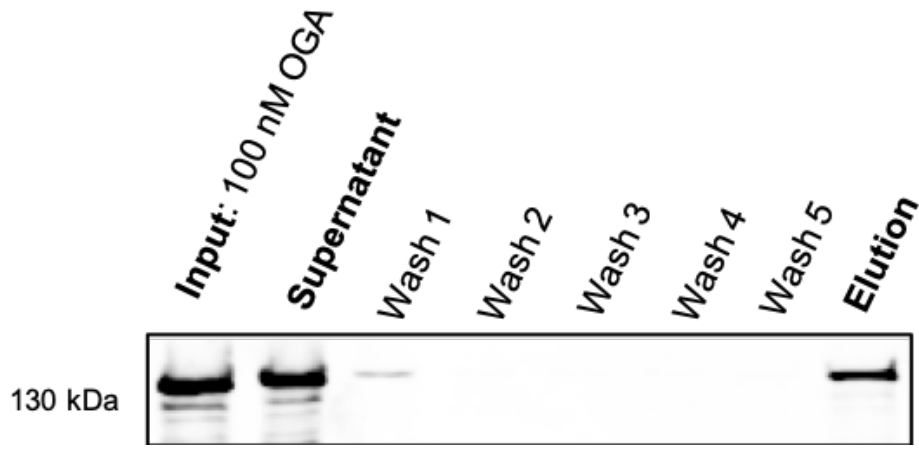

Supplemental Figure 14. Small scale validation experiment showing DNJNAc-6S-NBn-Biotin enables enrichment of recombinant hOGA as determined using immunoblot assay. 


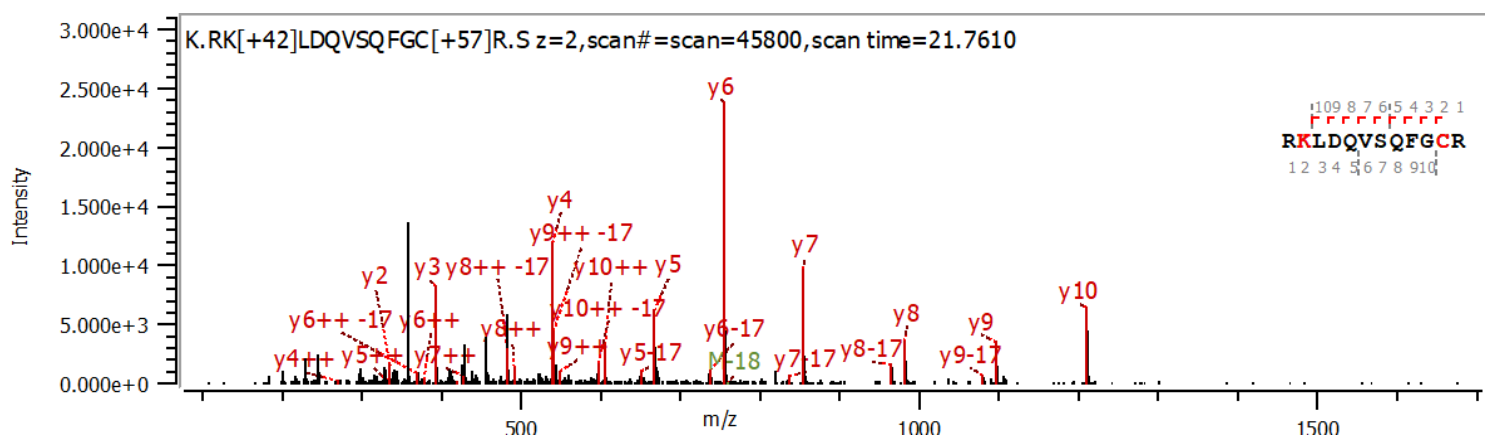

Supplemental Figure 15. MS/MS spectrum for acetylation identified at K157.

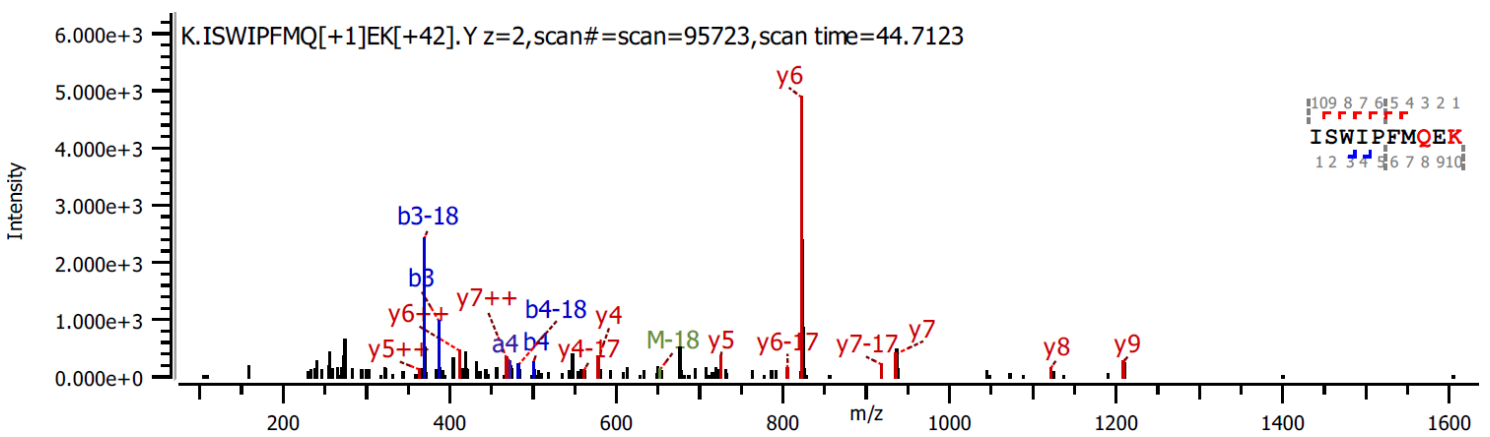

Supplemental Figure 16. MS/MS spectrum for acetylation identified at K805.

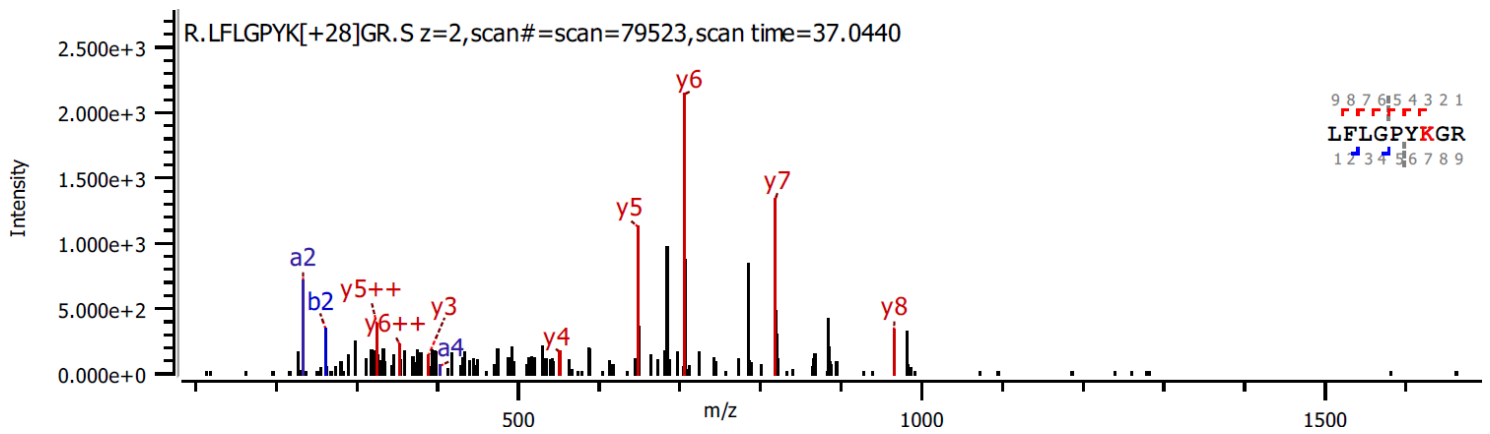

Supplemental Figure 17. MS/MS spectrum for formylation identified at K297. 


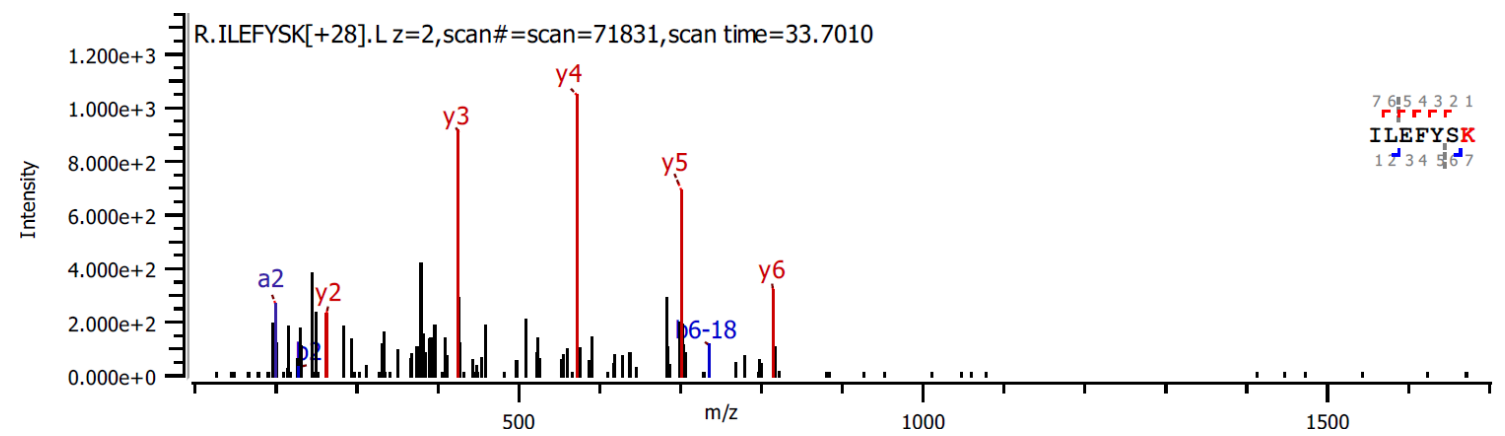

Supplemental Figure 18. MS/MS spectrum for formylation identified at K893.

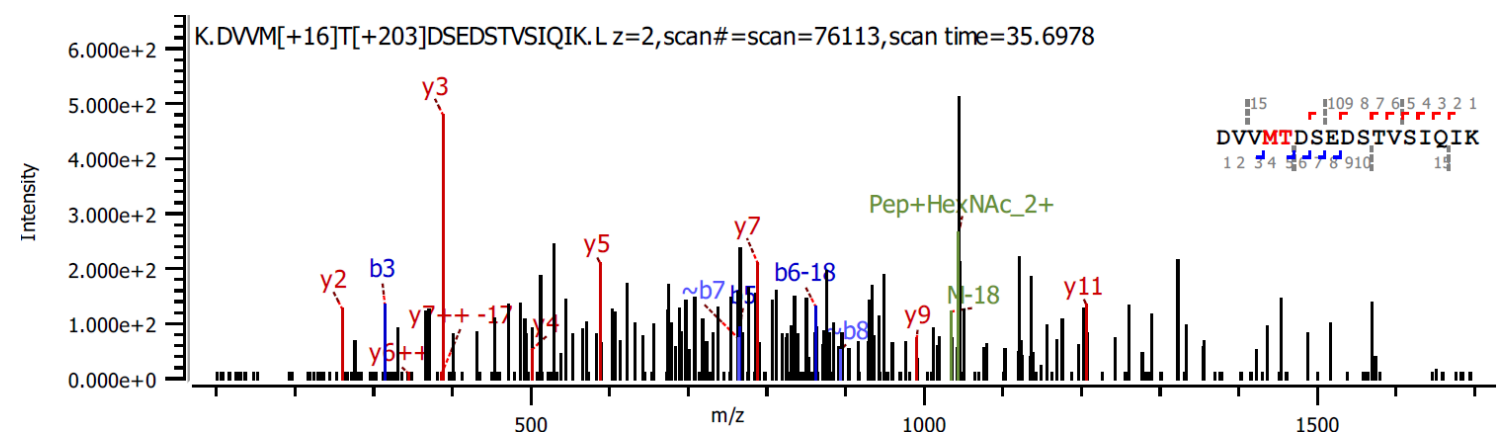

Supplemental Figure 19. MS/MS spectrum for O-GlcNAcylation identified at T346.

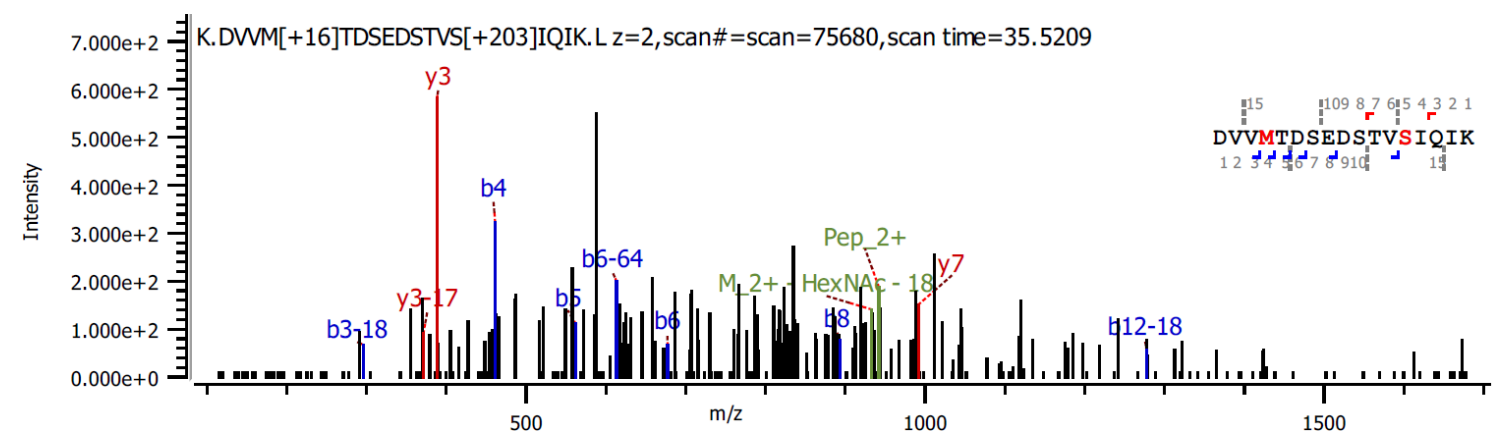

Supplemental Figure 20. MS/MS spectrum for O-GlcNAcylation identified at S354. 


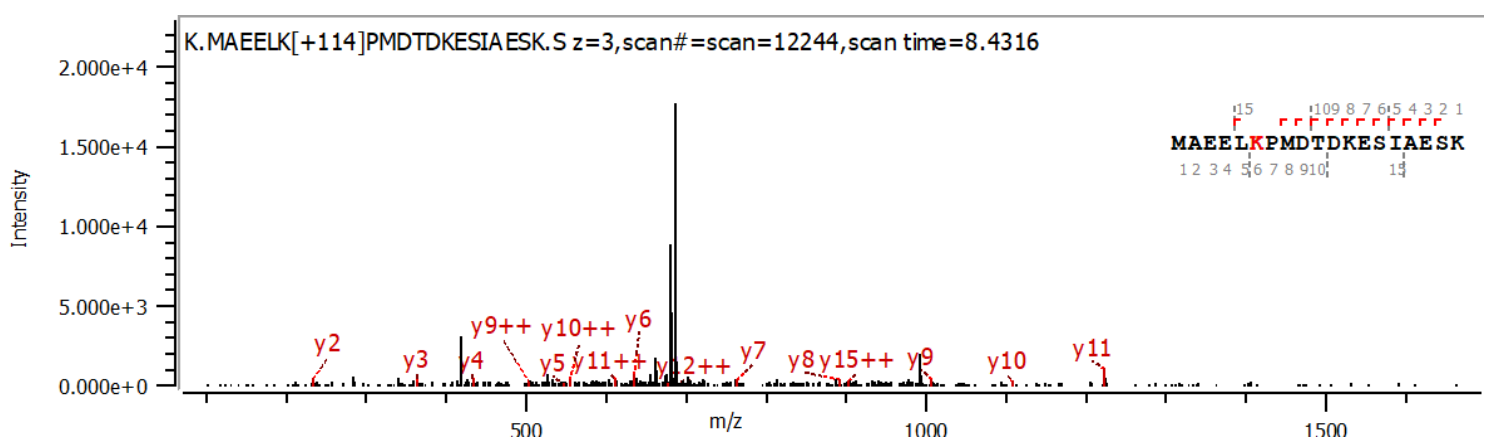

Supplemental Figure 21. MS/MS spectrum for N-ubiquitination identified at K497.

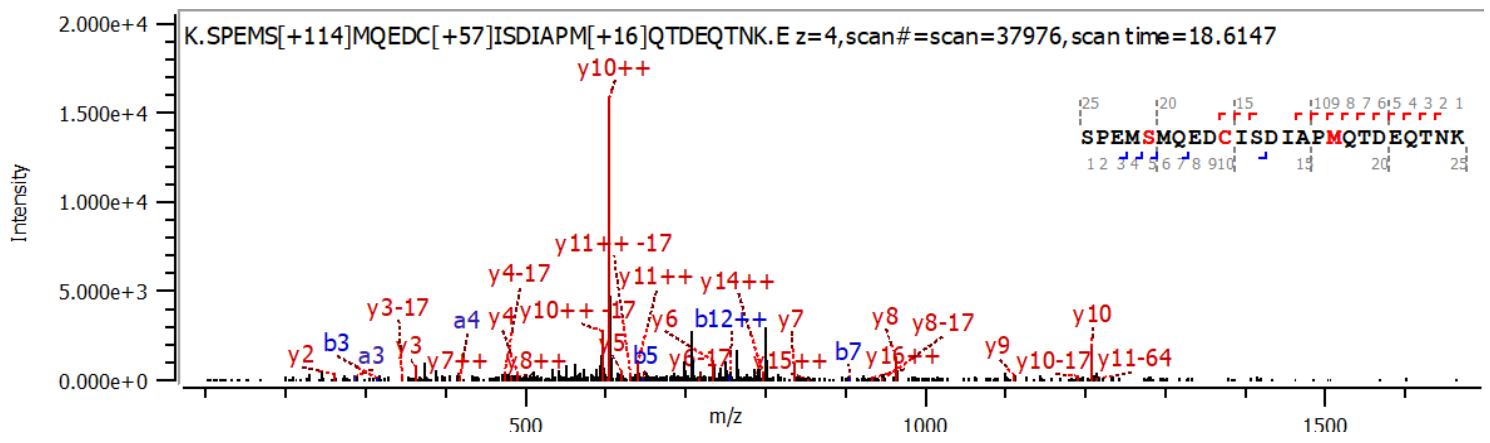

Supplemental Figure 22. MS/MS spectrum for O-ubiquitination identified at S515.

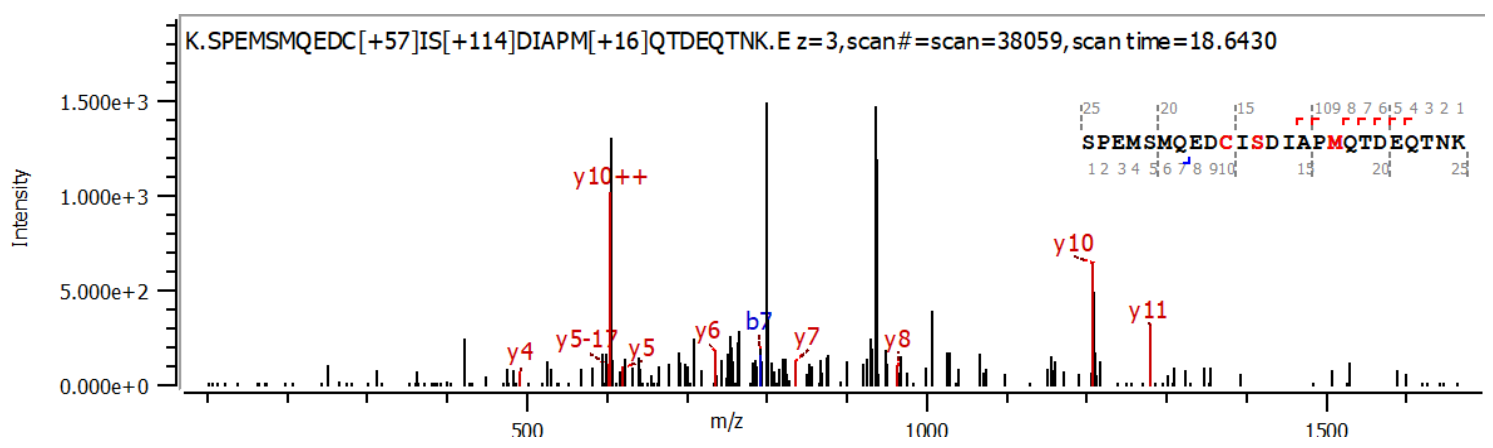

Supplemental Figure 23. MS/MS spectrum for O-ubiquitination identified at S522. 
Supplemental Table 3. Fragment ion series of mass spectra from acetylated Lys157 peptide.

\begin{tabular}{|c|c|c|c|c|c|c|c|c|c|c|c|c|c|c|c|c|c|}
\hline$\#$ & a calc. & $\begin{array}{c}\text { a } \\
\text { obs. }\end{array}$ & $\begin{array}{c}\text { a } \\
\text { delta }\end{array}$ & b calc. & $\begin{array}{c}\text { b } \\
\text { obs. }\end{array}$ & $\begin{array}{c}\mathbf{b} \\
\text { delta }\end{array}$ & $\begin{array}{l}\text { b-18 } \\
\text { calc. }\end{array}$ & $\begin{array}{l}\text { b-18 } \\
\text { obs. }\end{array}$ & $\begin{array}{l}\text { b-18 } \\
\text { delta }\end{array}$ & Seq. & y calc. & y obs. & y delta & y++ calc. & y++ obs. & y++ delta & \# \\
\hline 1 & 129.1135 & & & 157.1084 & & & 139.0978 & & & $\mathbf{R}$ & & & & & & & 12 \\
\hline 2 & 299.219 & & & 327.2139 & & & 309.2033 & & & K & 1379.6736 & & & 690.3404 & & & 11 \\
\hline 3 & 412.3031 & & & 440.298 & & & 422.2874 & & & $\mathbf{L}$ & 1209.5681 & 1209.5676 & -0.0005 & 605.2877 & 605.2874 & -0.0002 & 10 \\
\hline 4 & 527.33 & & & 555.3249 & & & 537.3143 & & & D & 1096.484 & 1096.4829 & -0.0011 & 548.7456 & 548.743 & -0.0027 & 9 \\
\hline 5 & 655.3886 & & & 683.3835 & & & 665.3729 & & & $\mathbf{Q}$ & 981.4571 & 981.4567 & -0.0004 & 491.2322 & 491.2281 & -0.0041 & 8 \\
\hline 6 & 754.457 & & & 782.4519 & & & 764.4413 & & & v & 853.3985 & 853.3932 & -0.0053 & 427.2029 & 427.1967 & -0.0062 & 7 \\
\hline 7 & 841.489 & & & 869.4839 & & & 851.4734 & & & $s$ & 754.3301 & 754.328 & -0.002 & 377.6687 & 377.664 & -0.0047 & 6 \\
\hline 8 & 969.5476 & & & 997.5425 & & & 979.5319 & & & $\mathbf{Q}$ & 667.298 & 667.2953 & -0.0027 & 334.1527 & 334.1502 & -0.0025 & 5 \\
\hline 9 & 1116.616 & & & 1144.6109 & & & 1126.6004 & & & $\mathbf{F}$ & 539.2395 & 539.2367 & -0.0028 & 270.1234 & 270.1241 & 0.0007 & 4 \\
\hline 10 & 1173.6375 & & & 1201.6324 & & & 1183.6218 & & & G & 392.1711 & 392.1692 & -0.0019 & 196.5892 & & & 3 \\
\hline 11 & 1333.6681 & & & 1361.663 & & & 1343.6525 & & & C & 335.1496 & 335.1479 & -0.0017 & 168.0784 & & & 2 \\
\hline 12 & & & & & & & & & & $\mathbf{R}$ & 175.119 & & & 88.0631 & & & 1 \\
\hline
\end{tabular}


Supplemental Table 4. Fragment ion series of mass spectra from acetylated Lys805 peptide.

\begin{tabular}{|c|c|c|c|c|c|c|c|c|c|c|c|c|c|c|c|c|c|}
\hline \# & a calc. & a obs. & $\begin{array}{c}a \\
\text { delta }\end{array}$ & b calc. & b obs. & $\begin{array}{c}\text { b } \\
\text { delta }\end{array}$ & $\begin{array}{l}\text { b-18 } \\
\text { calc. }\end{array}$ & $\begin{array}{l}\text { b-18 } \\
\text { obs. }\end{array}$ & $\begin{array}{l}\text { b-18 } \\
\text { delta }\end{array}$ & Seq. & y calc. & y obs. & y delta & y++ calc. & y++ obs. & $\begin{array}{c}\text { y++ } \\
\text { delta }\end{array}$ & $\#$ \\
\hline 1 & 86.0964 & & & 114.0913 & & & 96.0808 & & & 1 & & & & & & & 10 \\
\hline 2 & 173.1285 & & & 201.1234 & & & 183.1128 & & & $\mathrm{~s}$ & 1208.5656 & 1208.5888 & 0.0232 & 604.7864 & & & 9 \\
\hline 3 & 359.2078 & 359.2091 & 0.0013 & 387.2027 & 387.1984 & -0.0042 & 369.1921 & 369.1891 & -0.0031 & w & 1121.5336 & 1121.5592 & 0.0256 & 561.2704 & & & 8 \\
\hline 4 & 472.2918 & 472.2934 & 0.0016 & 500.2867 & 500.2866 & -0.0002 & 482.2762 & & & I & 935.4543 & 935.4616 & 0.0073 & 468.2308 & & & 7 \\
\hline 5 & 569.3446 & & & 597.3395 & & & 579.3289 & & & $\mathbf{P}$ & 822.3702 & 822.3776 & 0.0074 & 411.6887 & 411.6924 & 0.0036 & 6 \\
\hline 6 & 716.413 & & & 744.4079 & & & 726.3973 & & & $\mathbf{F}$ & 725.3174 & 725.3282 & 0.0107 & 363.1624 & & & 5 \\
\hline 7 & 847.4535 & & & 875.4484 & & & 857.4378 & & & M & 578.249 & 578.2581 & 0.009 & 289.6282 & & & 4 \\
\hline 8 & 976.4961 & & & 1004.491 & & & 986.4804 & & & $\mathbf{Q}$ & 447.2086 & & & 224.1079 & & & 3 \\
\hline 9 & 1105.5387 & & & 1133.5336 & & & 1115.523 & & & $\mathrm{E}$ & 318.166 & & & 159.5866 & & & 2 \\
\hline 10 & & & & & & & & & & K & 189.1234 & & & 95.0653 & & & 1 \\
\hline
\end{tabular}


Supplemental Table 5. Fragment ion series of mass spectra from formylated Lys297 peptide.

\begin{tabular}{|c|c|c|c|c|c|c|c|c|c|c|c|c|c|c|c|c|c|}
\hline \# & a calc. & a obs. & a delta & b calc. & b obs. & $\begin{array}{c}\text { b } \\
\text { delta }\end{array}$ & $\begin{array}{l}\text { b-18 } \\
\text { calc. }\end{array}$ & $\begin{array}{l}\text { b-18 } \\
\text { obs. }\end{array}$ & $\begin{array}{l}\text { b-18 } \\
\text { delta }\end{array}$ & Seq. & y calc. & y obs. & y delta & y++ calc. & y++ obs. & $\mathrm{y}++$ delta & $\#$ \\
\hline 1 & 86.0964 & & & 114.0913 & & & 96.0808 & & & $\mathrm{~L}$ & & & & & & & 9 \\
\hline 2 & 233.1648 & 233.1635 & -0.0014 & 261.1597 & 261.1567 & -0.0031 & 243.1492 & & & $\mathbf{F}$ & 965.5203 & 965.5163 & -0.004 & 483.2638 & & & 8 \\
\hline 3 & 346.2489 & & & 374.2438 & & & 356.2332 & & & L & 818.4519 & 818.4503 & -0.0016 & 409.7296 & & & 7 \\
\hline 4 & 403.2704 & 403.2642 & -0.0061 & 431.2653 & & & 413.2547 & & & G & 705.3678 & 705.3676 & -0.0002 & 353.1876 & 353.1834 & -0.0042 & 6 \\
\hline 5 & 500.3231 & & & 528.318 & & & 510.3075 & & & $\mathbf{P}$ & 648.3464 & 648.3451 & -0.0013 & 324.6768 & 324.6788 & 0.002 & 5 \\
\hline 6 & 663.3864 & & & 691.3814 & & & 673.3708 & & & Y & 551.2936 & 551.2972 & 0.0036 & 276.1504 & & & 4 \\
\hline 7 & 819.4763 & & & 847.4712 & & & 829.4607 & & & K & 388.2303 & 388.2242 & -0.0061 & 194.6188 & & & 3 \\
\hline 8 & 876.4978 & & & 904.4927 & & & 886.4821 & & & G & 232.1404 & & & 116.5738 & & & 2 \\
\hline 9 & & & & & & & & & & $\mathbf{R}$ & 175.119 & & & 88.0631 & & & 1 \\
\hline
\end{tabular}


Supplemental Table 6. Fragment ion series of mass spectra from formylated Lys893 peptide.

\begin{tabular}{|c|c|c|c|c|c|c|c|c|c|c|c|c|c|c|c|c|c|}
\hline \# & a calc. & a obs. & a delta & b calc. & b obs. & b delta & b-18 calc. & b-18 obs. & $\begin{array}{l}\text { b-18 } \\
\text { delta }\end{array}$ & Seq. & y calc. & y obs. & y delta & y++ calc. & $\begin{array}{l}\text { y++ } \\
\text { obs. }\end{array}$ & $\begin{array}{c}\text { y++ } \\
\text { delta }\end{array}$ & $\#$ \\
\hline 1 & 86.0964 & & & 114.0913 & & & 96.0808 & & & 1 & & & & & & & 7 \\
\hline 2 & 199.1805 & 199.183 & 0.0025 & 227.1754 & 227.1735 & -0.0019 & 209.1648 & & & L & 814.3981 & 814.3996 & 0.0015 & 407.7027 & & & 6 \\
\hline 3 & 328.2231 & & & 356.218 & & & 338.2074 & & & E & 701.3141 & 701.3176 & 0.0035 & 351.1607 & & & 5 \\
\hline 4 & 475.2915 & & & 503.2864 & & & 485.2758 & & & $\mathbf{F}$ & 572.2715 & 572.265 & -0.0064 & 286.6394 & & & 4 \\
\hline 5 & 638.3548 & & & 666.3497 & & & 648.3392 & & & $\mathrm{Y}$ & 425.2031 & 425.2002 & -0.0029 & 213.1052 & & & 3 \\
\hline 6 & 725.3868 & & & 753.3818 & & & 735.3712 & 735.3912 & 0.02 & $\mathrm{~s}$ & 262.1397 & 262.14 & 0.0003 & 131.5735 & & & 2 \\
\hline 7 & & & & & & & & & & K & 175.1077 & & & 88.0575 & & & 1 \\
\hline
\end{tabular}


Supplemental Table 7. Fragment ion series of mass spectra from O-GlcNAcylated Thr346 peptide.

\begin{tabular}{|c|c|c|c|c|c|c|c|c|c|c|c|c|c|c|c|c|c|}
\hline \# & a calc. & $\begin{array}{c}\text { a } \\
\text { obs. }\end{array}$ & $\begin{array}{c}\text { a } \\
\text { delta }\end{array}$ & b calc. & b obs. & b delta & b-18 calc. & $\begin{array}{l}\text { b-18 } \\
\text { obs. }\end{array}$ & $\begin{array}{l}\text { b-18 } \\
\text { delta }\end{array}$ & Seq. & y calc. & y obs. & y delta & $\begin{array}{l}\text { y++ } \\
\text { calc. }\end{array}$ & y++ obs. & $\begin{array}{c}\text { y++ } \\
\text { delta }\end{array}$ & $\#$ \\
\hline 1 & 88.0393 & & & 116.0342 & & & 98.0237 & & & D & & & & & & & 17 \\
\hline 2 & 187.1077 & & & 215.1026 & & & 197.0921 & & & v & 1970.9474 & & & 985.9773 & & & 16 \\
\hline 3 & 286.1761 & & & 314.171 & 314.1609 & -0.0101 & 296.1605 & & & v & 1871.879 & & & 936.4431 & & & 15 \\
\hline 4 & 433.2115 & & & 461.2064 & & & 443.1959 & & & M & 1772.8106 & & & 886.9089 & & & 14 \\
\hline 5 & 737.3386 & & & 765.3335 & 765.3178 & -0.0157 & 747.3229 & & & $T$ & 1625.7752 & & & 813.3912 & & & 13 \\
\hline 6 & 852.3655 & & & 880.3604 & & & 862.3499 & 862.3284 & -0.0214 & D & 1321.6482 & & & 661.3277 & & & 12 \\
\hline 7 & 939.3975 & & & 967.3925 & & & 949.3819 & & & $s$ & 1206.6212 & 1206.6213 & 0.0001 & 603.8142 & & & 11 \\
\hline 8 & 1068.4401 & & & 1096.435 & & & 1078.4245 & & & E & 1119.5892 & & & 560.2982 & & & 10 \\
\hline 9 & 1183.4671 & & & 1211.462 & & & 1193.4514 & & & D & 990.5466 & 990.5421 & -0.0045 & 495.7769 & & & 9 \\
\hline 10 & 1270.4991 & & & 1298.494 & & & 1280.4835 & & & s & 875.5197 & & & 438.2635 & & & 8 \\
\hline 11 & 1371.5468 & & & 1399.5417 & & & 1381.5311 & & & $\begin{array}{ll}T \\
\end{array}$ & 788.4876 & 788.4909 & 0.0033 & 394.7474 & & & 7 \\
\hline 12 & 1470.6152 & & & 1498.6101 & & & 1480.5995 & & & v & 687.4399 & & & 344.2236 & 344.237 & 0.0134 & 6 \\
\hline 13 & 1557.6472 & & & 1585.6421 & & & 1567.6316 & & & s & 588.3715 & 588.3682 & -0.0033 & 294.6894 & & & 5 \\
\hline 14 & 1670.7313 & & & 1698.7262 & & & 1680.7156 & & & 1 & 501.3395 & 501.3279 & -0.0116 & 251.1734 & & & 4 \\
\hline 15 & 1798.7899 & & & 1826.7848 & & & 1808.7742 & & & Q & 388.2554 & 388.2561 & 0.0006 & 194.6314 & & & 3 \\
\hline 16 & 1911.8739 & & & 1939.8688 & & & 1921.8583 & & & 1 & 260.1969 & 260.1967 & -0.0002 & 130.6021 & & & 2 \\
\hline 17 & & & & & & & & & & K & 147.1128 & & & 74.06 & & & 1 \\
\hline
\end{tabular}


Supplemental Table 8. Fragment ion series of mass spectra from O-GlcNAcylated Ser354 peptide.

\begin{tabular}{|c|c|c|c|c|c|c|c|c|c|c|c|c|c|c|c|c|c|}
\hline \# & a calc. & $\begin{array}{c}\text { a } \\
\text { obs. }\end{array}$ & $\begin{array}{c}\text { a } \\
\text { delta }\end{array}$ & b calc. & b obs. & b delta & b-18 calc. & b-18 obs. & $\begin{array}{l}\text { b-18 } \\
\text { delta }\end{array}$ & Seq. & y calc. & y obs. & $\begin{array}{c}y \\
\text { delta }\end{array}$ & $\begin{array}{l}\text { y++ } \\
\text { calc. }\end{array}$ & $\begin{array}{l}y++ \\
\text { obs. }\end{array}$ & $\begin{array}{c}\mathrm{y++} \\
\text { delta }\end{array}$ & $\#$ \\
\hline 1 & 88.0393 & & & 116.0342 & & & 98.0237 & & & D & & & & & & & 17 \\
\hline 2 & 187.1077 & & & 215.1026 & & & 197.0921 & & & v & 1970.9474 & & & 985.9773 & & & 16 \\
\hline 3 & 286.1761 & & & 314.171 & & & 296.1605 & 296.1571 & -0.0034 & v & 1871.879 & & & 936.4431 & & & 15 \\
\hline 4 & 433.2115 & & & 461.2064 & 461.1902 & -0.0162 & 443.1959 & & & M & 1772.8106 & & & 886.9089 & & & 14 \\
\hline 5 & 534.2592 & & & 562.2541 & 562.2681 & 0.014 & 544.2436 & & & $T$ & 1625.7752 & & & 813.3912 & & & 13 \\
\hline 6 & 649.2861 & & & 677.2811 & 677.2795 & -0.0016 & 659.2705 & & & D & 1524.7275 & & & 762.8674 & & & 12 \\
\hline 7 & 736.3182 & & & 764.3131 & & & 746.3025 & & & $\mathbf{s}$ & 1409.7006 & & & 705.3539 & & & 11 \\
\hline 8 & 865.3608 & & & 893.3557 & 893.3476 & -0.0081 & 875.3451 & & & E & 1322.6686 & & & 661.8379 & & & 10 \\
\hline 9 & 980.3877 & & & 1008.3826 & & & 990.3721 & & & D & 1193.626 & & & 597.3166 & & & 9 \\
\hline 10 & 1067.4197 & & & 1095.4146 & & & 1077.4041 & & & $\mathrm{~s}$ & 1078.599 & & & 539.8031 & & & 8 \\
\hline 11 & 1168.4674 & & & 1196.4623 & & & 1178.4518 & & & $T$ & 991.567 & 991.5449 & -0.0221 & 496.2871 & & & 7 \\
\hline 12 & 1267.5358 & & & 1295.5307 & & & 1277.5202 & 1277.5149 & -0.0052 & v & 890.5193 & & & 445.7633 & & & 6 \\
\hline 13 & 1557.6472 & & & 1585.6421 & & & 1567.6316 & & & $\mathrm{~s}$ & 791.4509 & & & 396.2291 & & & 5 \\
\hline 14 & 1670.7313 & & & 1698.7262 & & & 1680.7156 & & & 1 & 501.3395 & & & 251.1734 & & & 4 \\
\hline 15 & 1798.7899 & & & 1826.7848 & & & 1808.7742 & & & Q & 388.2554 & 388.2532 & -0.0022 & 194.6314 & & & 3 \\
\hline 16 & 1911.8739 & & & 1939.8688 & & & 1921.8583 & & & 1 & 260.1969 & & & 130.6021 & & & 2 \\
\hline 17 & & & & & & & & & & K & 147.1128 & & & 74.06 & & & 1 \\
\hline
\end{tabular}


Supplemental Table 9. Fragment ion series of mass spectra from N-ubiquitinated Lys497 peptide.

\begin{tabular}{|c|c|c|c|c|c|c|c|c|c|c|c|c|c|c|c|c|c|}
\hline$\#$ & a calc. & a obs. & $\begin{array}{c}\text { a } \\
\text { delta }\end{array}$ & b calc. & b obs. & $\begin{array}{c}\text { b } \\
\text { delta }\end{array}$ & b++ calc. & $\begin{array}{l}\text { b+++ } \\
\text { obs. }\end{array}$ & $\begin{array}{c}\text { b++ } \\
\text { delta }\end{array}$ & Seq. & y calc. & y obs. & $\begin{array}{c}\text { y } \\
\text { delta }\end{array}$ & y++ calc. & y++ obs. & $\begin{array}{c}\text { y++ } \\
\text { delta }\end{array}$ & $\#$ \\
\hline 1 & 104.0528 & & & 132.0478 & & & 66.5275 & & & $M$ & & & & & & & 19 \\
\hline 2 & 175.09 & & & 203.0849 & & & 102.0461 & & & A & 2135.0172 & & & 1068.0122 & & & 18 \\
\hline 3 & 304.1325 & & & 332.1275 & & & 166.5674 & & & E & 2063.9801 & & & 1032.4937 & & & 17 \\
\hline 4 & 433.1751 & & & 461.17 & & & 231.0887 & & & E & 1934.9375 & & & 967.9724 & & & 16 \\
\hline 5 & 546.2592 & & & 574.2541 & & & 287.6307 & & & L & 1805.8949 & & & 903.4511 & 903.4276 & -0.0235 & 15 \\
\hline 6 & 788.3971 & & & 816.392 & & & 408.6996 & & & $\mathrm{~K}$ & 1692.8109 & & & 846.9091 & & & 14 \\
\hline 7 & 885.4498 & & & 913.4448 & & & 457.226 & & & $\mathbf{P}$ & 1450.673 & & & 725.8401 & & & 13 \\
\hline 8 & 1016.4903 & & & 1044.4852 & & & 522.7463 & & & M & 1353.6202 & & & 677.3137 & 677.3246 & 0.0109 & 12 \\
\hline 9 & 1131.5173 & & & 1159.5122 & & & 580.2597 & & & D & 1222.5797 & 1222.5854 & 0.0057 & 611.7935 & 611.7944 & 0.0009 & 11 \\
\hline 10 & 1232.5649 & & & 1260.5599 & & & 630.7836 & & & $T$ & 1107.5528 & 1107.5622 & 0.0094 & 554.28 & 554.2744 & -0.0056 & 10 \\
\hline 11 & 1347.5919 & & & 1375.5868 & & & 688.297 & & & D & 1006.5051 & 1006.5058 & 0.0007 & 503.7562 & 503.7531 & -0.0031 & 9 \\
\hline 12 & 1475.6868 & & & 1503.6818 & & & 752.3445 & & & K & 891.4782 & 891.4658 & -0.0123 & 446.2427 & & & 8 \\
\hline 13 & 1604.7294 & & & 1632.7243 & & & 816.8658 & & & E & 763.3832 & 763.3694 & -0.0138 & 382.1952 & & & 7 \\
\hline 14 & 1691.7615 & & & 1719.7564 & & & 860.3818 & & & $s$ & 634.3406 & 634.3441 & 0.0034 & 317.6739 & & & 6 \\
\hline 15 & 1804.8455 & & & 1832.8404 & & & 916.9239 & & & 1 & 547.3086 & 547.3073 & -0.0013 & 274.1579 & & & 5 \\
\hline 16 & 1875.8826 & & & 1903.8775 & & & 952.4424 & & & A & 434.2245 & 434.2194 & -0.0052 & 217.6159 & & & 4 \\
\hline 17 & 2004.9252 & & & 2032.9201 & & & 1016.9637 & & & E & 363.1874 & 363.183 & -0.0045 & 182.0973 & & & 3 \\
\hline 18 & 2091.9573 & & & 2119.9522 & & & 1060.4797 & & & $\mathrm{~s}$ & 234.1448 & 234.1478 & 0.003 & 117.5761 & & & 2 \\
\hline 19 & & & & & & & & & & K & 147.1128 & & & 74.06 & & & 1 \\
\hline
\end{tabular}


Supplemental Table 10. Fragment ion series of mass spectra from O-ubiquitinated Ser515 peptide.

\begin{tabular}{|c|c|c|c|c|c|c|c|c|c|c|c|c|c|c|c|c|c|}
\hline \# & a calc. & a obs. & delta & b calc. & b obs. & $\stackrel{b}{b}$ & b++ calc. & $\begin{array}{l}\text { b++ } \\
\text { obs. }\end{array}$ & $\begin{array}{c}\text { b++ } \\
\text { delta }\end{array}$ & Seq. & y calc. & y obs. & delta & y++ calc. & y++ obs. & $\begin{array}{c}\text { y++ } \\
\text { delta }\end{array}$ & $\#$ \\
\hline 1 & 60.0444 & & & 88.0393 & & & 44.5233 & & & S & & & & & & & 25 \\
\hline 2 & 157.0972 & & & 185.0921 & & & 93.0497 & & & $\mathbf{P}$ & 2928.2002 & & & 1464.6037 & & & 24 \\
\hline 3 & 286.1397 & 286.1367 & -0.0031 & 314.1347 & 314.1301 & -0.0046 & 157.571 & & & E & 2831.1474 & & & 1416.0773 & & & 23 \\
\hline 4 & 417.1802 & 417.1868 & 0.0066 & 445.1751 & & & 223.0912 & & & M & 2702.1048 & & & 1351.556 & & & 22 \\
\hline 5 & 618.2552 & & & 646.2501 & 646.2548 & 0.0047 & 323.6287 & & & s & 2571.0643 & & & 1286.0358 & & & 21 \\
\hline 6 & 749.2957 & & & 777.2906 & & & 389.1489 & & & M & 2369.9894 & & & 1185.4983 & & & 20 \\
\hline 7 & 877.3542 & & & 905.3492 & 905.3553 & 0.0062 & 453.1782 & & & $\mathbf{Q}$ & 2238.9489 & & & 1119.9781 & & & 19 \\
\hline 8 & 1006.3968 & & & 1034.3917 & & & 517.6995 & & & E & 2110.8903 & & & 1055.9488 & & & 18 \\
\hline 9 & 1121.4238 & & & 1149.4187 & & & 575.213 & & & D & 1981.8477 & & & 991.4275 & & & 17 \\
\hline 10 & 1281.4544 & & & 1309.4493 & & & 655.2283 & & & C & 1866.8208 & & & 933.914 & 933.9101 & -0.004 & 16 \\
\hline 11 & 1394.5385 & & & 1422.5334 & & & 711.7703 & & & I & 1706.7901 & & & 853.8987 & 853.9253 & 0.0265 & 15 \\
\hline 12 & 1481.5705 & & & 1509.5654 & & & 755.2863 & 755.3037 & 0.0173 & $S$ & 1593.7061 & & & 797.3567 & 797.3519 & -0.0047 & 14 \\
\hline 13 & 1596.5974 & & & 1624.5924 & & & 812.7998 & & & D & 1506.6741 & & & 753.8407 & & & 13 \\
\hline 14 & 1709.6815 & & & 1737.6764 & & & 869.3418 & & & 1 & 1391.6471 & & & 696.3272 & & & 12 \\
\hline 15 & 1780.7186 & & & 1808.7135 & & & 904.8604 & & & A & 1278.5631 & & & 639.7852 & 639.7825 & -0.0026 & 11 \\
\hline 16 & 1877.7714 & & & 1905.7663 & & & 953.3868 & & & $\mathbf{P}$ & 1207.5259 & 1207.5258 & -0.0002 & 604.2666 & 604.2647 & -0.0019 & 10 \\
\hline 17 & 2024.8068 & & & 2052.8017 & & & 1026.9045 & & & $M$ & 1110.4732 & 1110.4827 & 0.0095 & 555.7402 & & & 9 \\
\hline 18 & 2152.8654 & & & 2180.8603 & & & 1090.9338 & & & $\mathbf{Q}$ & 963.4378 & 963.439 & 0.0013 & 482.2225 & 482.2222 & -0.0003 & 8 \\
\hline 19 & 2253.913 & & & 2281.9079 & & & 1141.4576 & & & $\mathbf{T}$ & 835.3792 & 835.3736 & -0.0056 & 418.1932 & 418.1956 & 0.0024 & 7 \\
\hline 20 & 2368.94 & & & 2396.9349 & & & 1198.9711 & & & D & 734.3315 & 734.3222 & -0.0093 & 367.6694 & & & 6 \\
\hline 21 & 2497.9826 & & & 2525.9775 & & & 1263.4924 & & & E & 619.3046 & 619.2951 & -0.0095 & 310.1559 & & & 5 \\
\hline 22 & 2626.0411 & & & 2654.0361 & & & 1327.5217 & & & $\mathbf{Q}$ & 490.262 & 490.2578 & -0.0042 & 245.6346 & & & 4 \\
\hline 23 & 2727.0888 & & & 2755.0837 & & & 1378.0455 & & & $\mathbf{T}$ & 362.2034 & 362.2053 & 0.0019 & 181.6053 & & & 3 \\
\hline 24 & 2841.1318 & & & 2869.1267 & & & 1435.067 & & & N & 261.1557 & 261.1527 & -0.0031 & 131.0815 & & & 2 \\
\hline 25 & & & & & & & & & & K & 147.1128 & & & 74.06 & & & 1 \\
\hline
\end{tabular}


Supplemental Table 11. Fragment ion series of mass spectra from O-ubiquitinated Ser522 peptide.

\begin{tabular}{|c|c|c|c|c|c|c|c|c|c|c|c|c|c|c|c|c|c|}
\hline$\#$ & a calc. & $\begin{array}{c}\text { a } \\
\text { obs. }\end{array}$ & $\begin{array}{c}a \\
\text { delta }\end{array}$ & b calc. & b obs. & $\begin{array}{c}\text { b } \\
\text { delta }\end{array}$ & b++ calc. & $\begin{array}{l}\text { b++ } \\
\text { obs. }\end{array}$ & $\begin{array}{c}\mathrm{b}++ \\
\text { delta }\end{array}$ & Seq. & y calc. & y obs. & $\begin{array}{c}y \\
\text { delta }\end{array}$ & y++ calc. & y++ obs. & $\begin{array}{c}\mathrm{y}++ \\
\text { delta }\end{array}$ & $\#$ \\
\hline 1 & 60.0444 & & & 88.0393 & & & 44.5233 & & & 5 & & & & & & & 25 \\
\hline 2 & 157.0972 & & & 185.0921 & & & 93.0497 & & & $\mathbf{P}$ & 2928.2002 & & & 1464.6037 & & & 24 \\
\hline 3 & 286.1397 & & & 314.1347 & & & 157.571 & & & E & 2831.1474 & & & 1416.0773 & & & 23 \\
\hline 4 & 417.1802 & & & 445.1751 & & & 223.0912 & & & $M$ & 2702.1048 & & & 1351.556 & & & 22 \\
\hline 5 & 504.2123 & & & 532.2072 & & & 266.6072 & & & s & 2571.0643 & & & 1286.0358 & & & 21 \\
\hline 6 & 635.2527 & & & 663.2476 & & & 332.1275 & & & M & 2484.0323 & & & 1242.5198 & & & 20 \\
\hline 7 & 763.3113 & & & 791.3062 & 791.3231 & 0.0169 & 396.1568 & & & $\mathbf{Q}$ & 2352.9918 & & & 1176.9995 & & & 19 \\
\hline 8 & 892.3539 & & & 920.3488 & & & 460.678 & & & E & 2224.9332 & & & 1112.9703 & & & 18 \\
\hline 9 & 1007.3808 & & & 1035.3758 & & & 518.1915 & & & D & 2095.8907 & & & 1048.449 & & & 17 \\
\hline 10 & 1167.4115 & & & 1195.4064 & & & 598.2068 & & & c & 1980.8637 & & & 990.9355 & & & 16 \\
\hline 11 & 1280.4955 & & & 1308.4905 & & & 654.7489 & & & I & 1820.8331 & & & 910.9202 & & & 15 \\
\hline 12 & 1481.5705 & & & 1509.5654 & & & 755.2863 & & & S & 1707.749 & & & 854.3781 & & & 14 \\
\hline 13 & 1596.5974 & & & 1624.5924 & & & 812.7998 & & & D & 1506.6741 & & & 753.8407 & & & 13 \\
\hline 14 & 1709.6815 & & & 1737.6764 & & & 869.3418 & & & 1 & 1391.6471 & & & 696.3272 & & & 12 \\
\hline 15 & 1780.7186 & & & 1808.7135 & & & 904.8604 & & & A & 1278.5631 & 1278.5622 & -0.0008 & 639.7852 & & & 11 \\
\hline 16 & 1877.7714 & & & 1905.7663 & & & 953.3868 & & & $\mathbf{P}$ & 1207.5259 & 1207.5212 & -0.0047 & 604.2666 & 604.2566 & -0.01 & 10 \\
\hline 17 & 2024.8068 & & & 2052.8017 & & & 1026.9045 & & & M & 1110.4732 & & & 555.7402 & & & 9 \\
\hline 18 & 2152.8654 & & & 2180.8603 & & & 1090.9338 & & & $\mathbf{Q}$ & 963.4378 & 963.4367 & -0.0011 & 482.2225 & & & 8 \\
\hline 19 & 2253.913 & & & 2281.9079 & & & 1141.4576 & & & $T$ & 835.3792 & 835.3679 & -0.0113 & 418.1932 & & & 7 \\
\hline 20 & 2368.94 & & & 2396.9349 & & & 1198.9711 & & & D & 734.3315 & 734.3225 & -0.009 & 367.6694 & & & 6 \\
\hline 21 & 2497.9826 & & & 2525.9775 & & & 1263.4924 & & & E & 619.3046 & 619.2927 & -0.0119 & 310.1559 & & & 5 \\
\hline 22 & 2626.0411 & & & 2654.0361 & & & 1327.5217 & & & $\mathbf{Q}$ & 490.262 & 490.2635 & 0.0015 & 245.6346 & & & 4 \\
\hline 23 & 2727.0888 & & & 2755.0837 & & & 1378.0455 & & & $T$ & 362.2034 & & & 181.6053 & & & 3 \\
\hline 24 & 2841.1318 & & & 2869.1267 & & & 1435.067 & & & N & 261.1557 & & & 131.0815 & & & 2 \\
\hline 25 & & & & & & & & & & к & 147.1128 & & & 74.06 & & & 1 \\
\hline
\end{tabular}




\section{Brain permeability in vivo of compound 16}

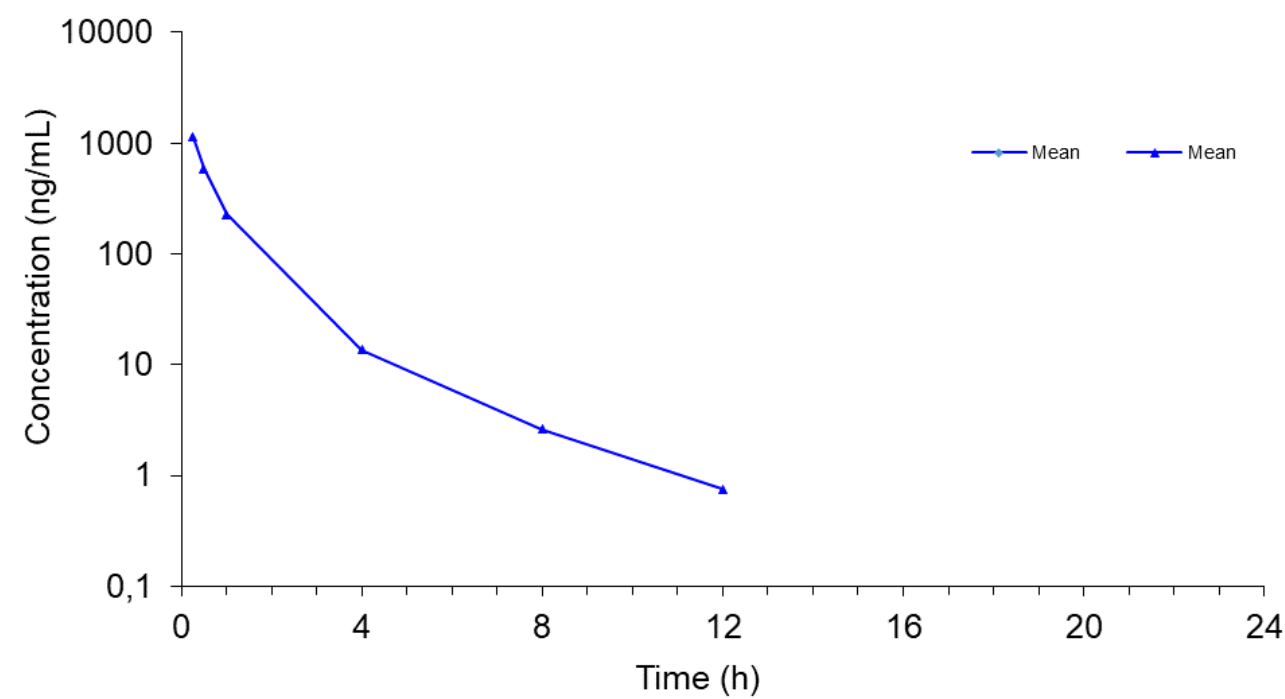

Supplemental Figure 24. Rat exposure in plasma from a single oral dose of 16 (3 mg/kg).

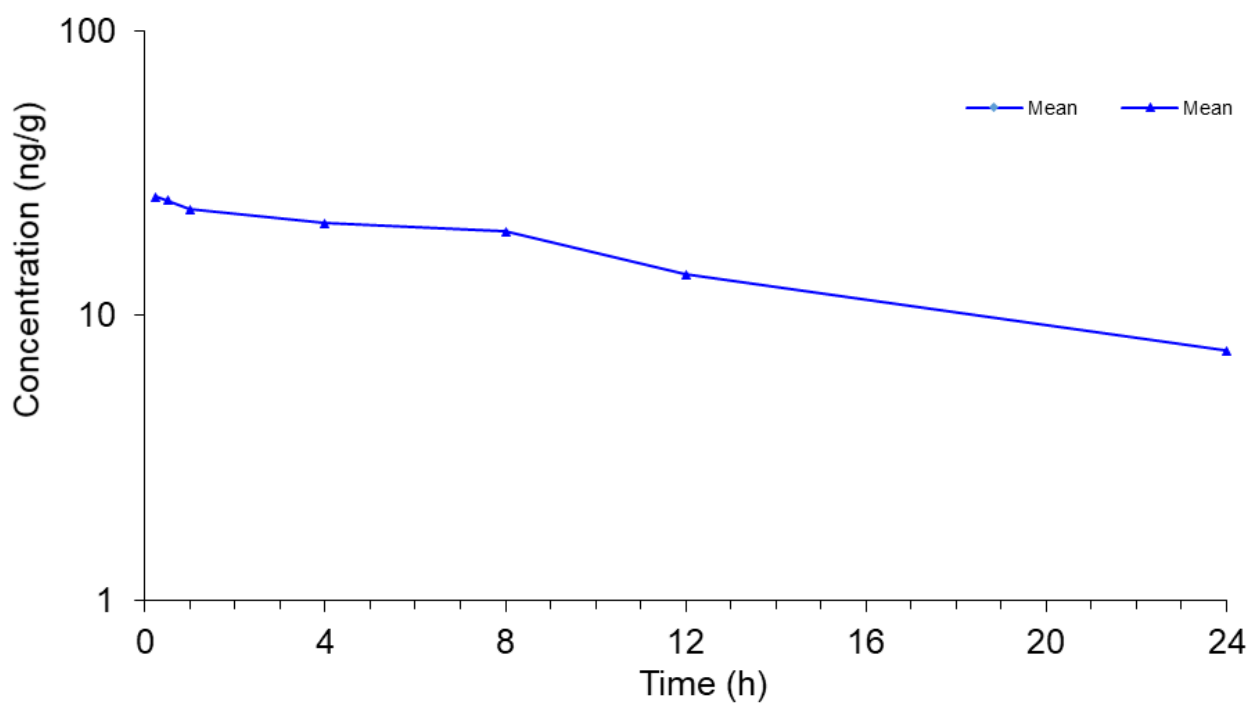

Supplemental Figure 25. Rat exposure in homogenized brain from a single oral dose of $\mathbf{1 6}$ (3 $\mathrm{mg} / \mathrm{kg}$ ). 


\section{Copies of ${ }^{1} \mathrm{H}$ and ${ }^{13} \mathrm{C}$ NMR and MS spectra}
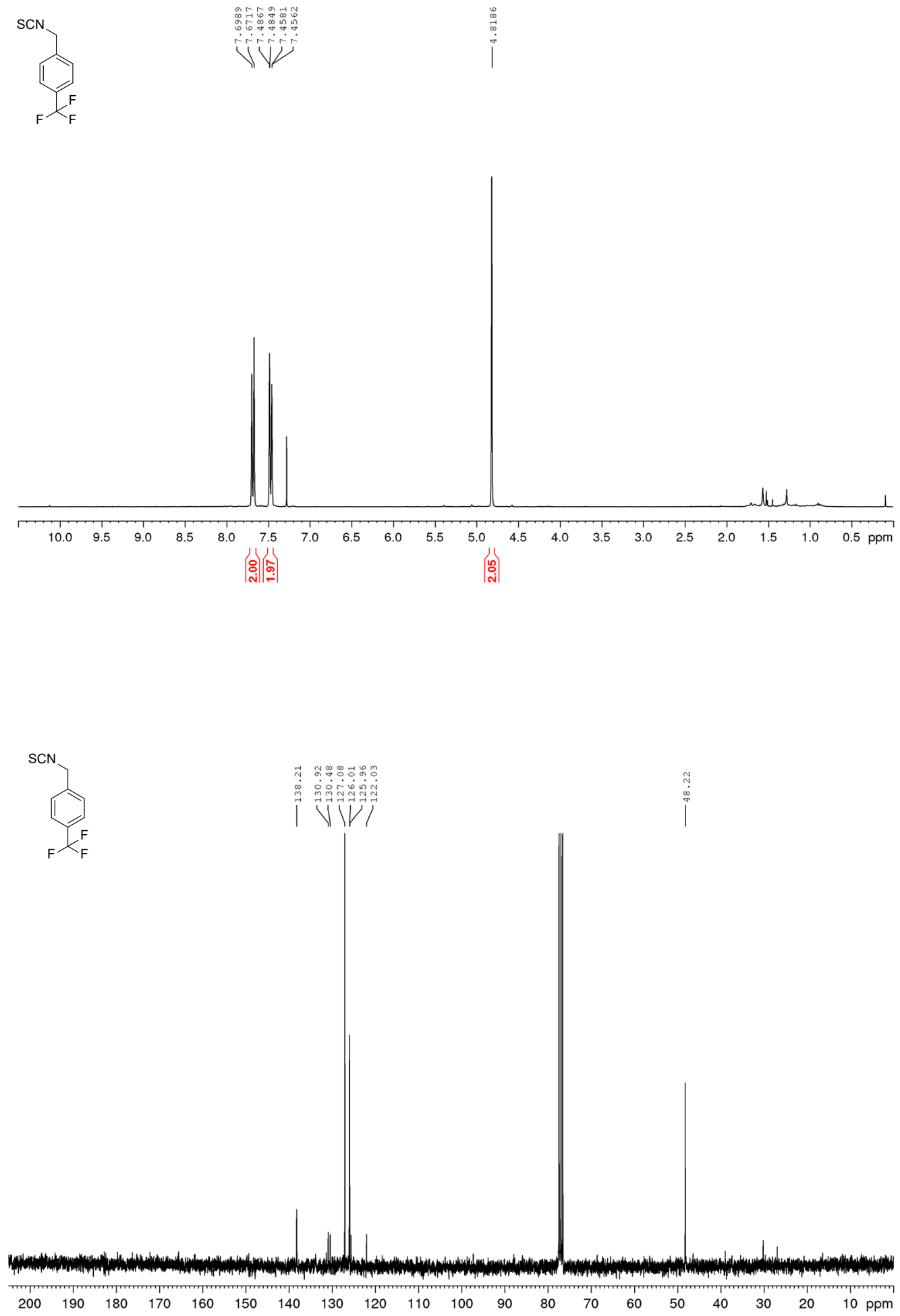

Supplemental Figure 26. ${ }^{1} \mathrm{H}$ NMR and ${ }^{13} \mathrm{C}$ NMR $\left(300 \mathrm{MHz}, 75.5 \mathrm{MHz} \mathrm{CDCl}_{3}\right)$ of TFBNCS 

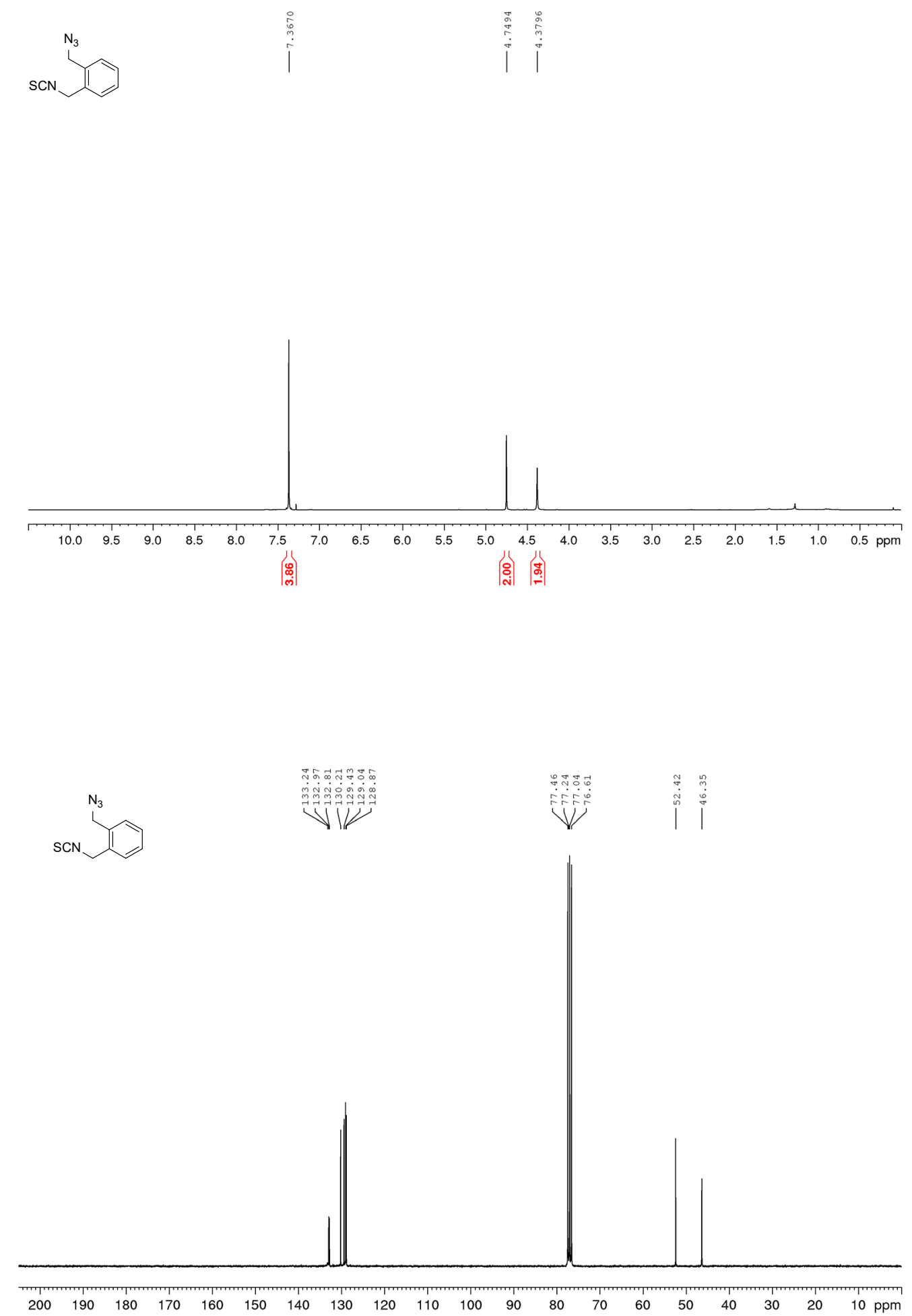

Supplemental Figure 27. ${ }^{1} \mathrm{H}$ NMR and ${ }^{13} \mathrm{C}$ NMR $\left(300 \mathrm{MHz}, 75.5 \mathrm{MHz} \mathrm{CDCl}_{3}\right.$ ) of $\boldsymbol{o}$ ABNCS 

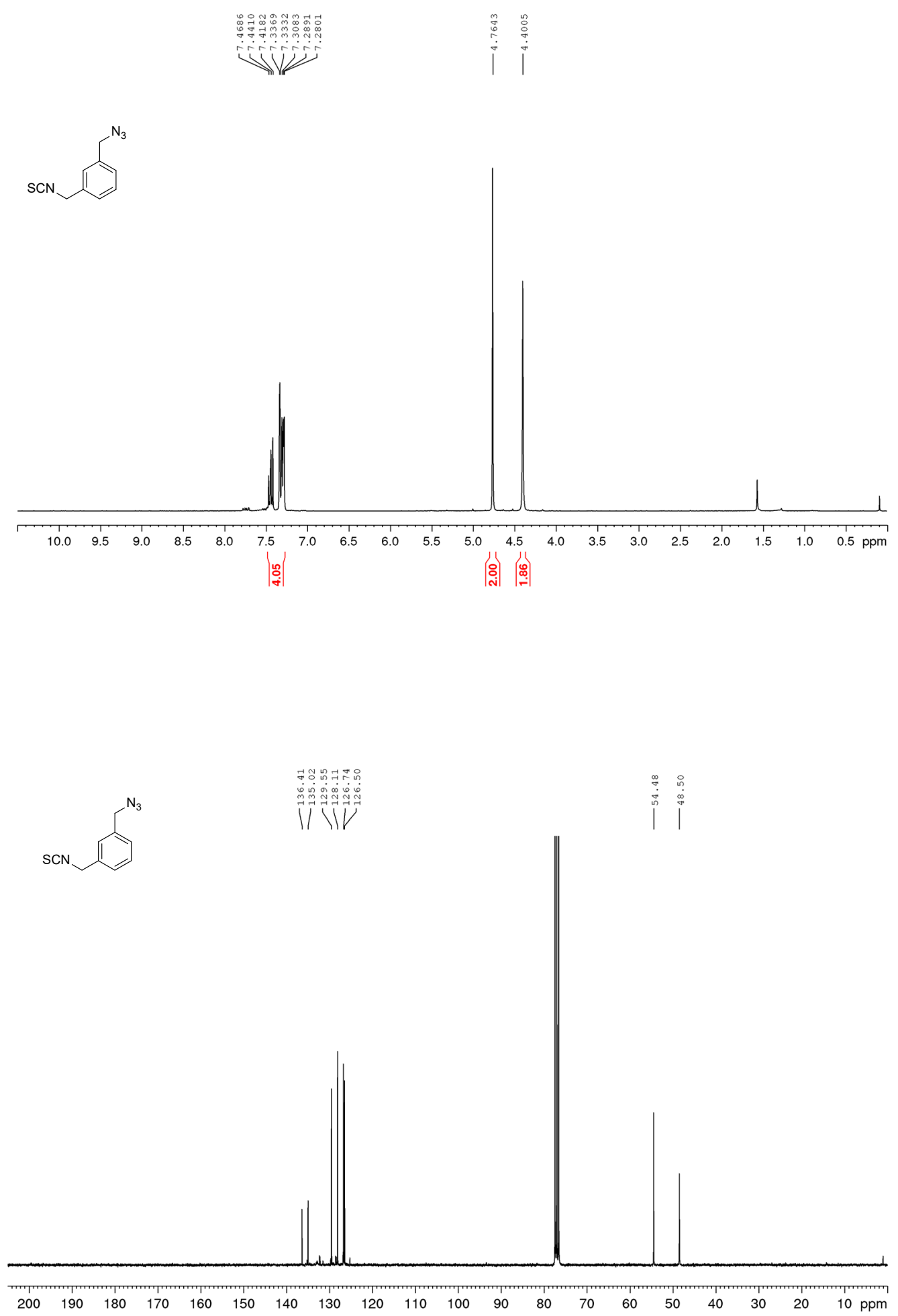

Supplemental Figure 28. ${ }^{1} \mathrm{H}$ NMR and ${ }^{13} \mathrm{C}$ NMR $\left(300 \mathrm{MHz}, 75.5 \mathrm{MHz} \mathrm{CDCl}_{3}\right)$ of $\boldsymbol{m}$ ABNCS 

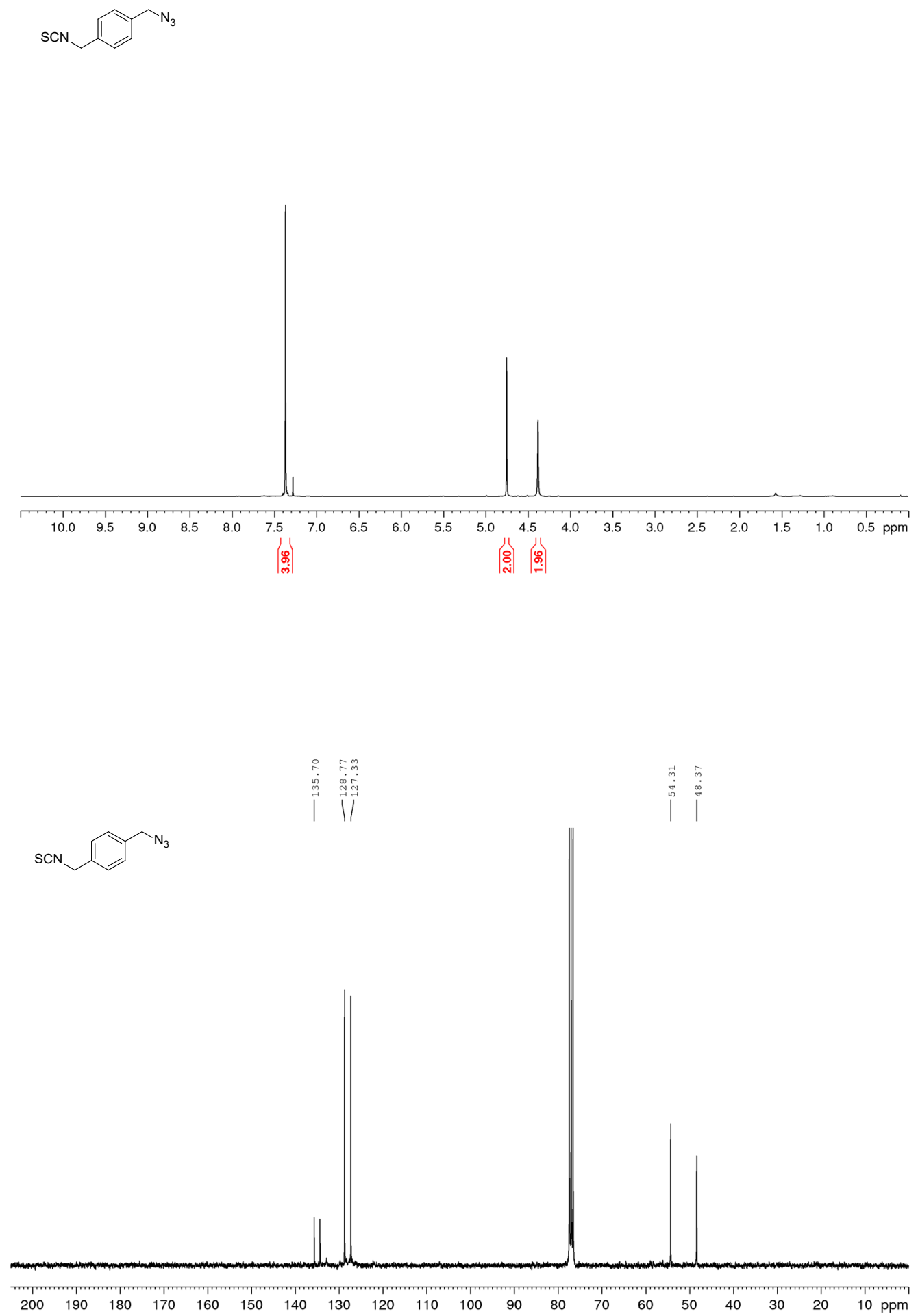

Supplemental Figure 29. ${ }^{1} \mathrm{H}$ NMR and ${ }^{13} \mathrm{C}$ NMR $\left(300 \mathrm{MHz}, 75.5 \mathrm{MHz} \mathrm{CDCl}_{3}\right)$ of $\boldsymbol{p}$ ABNCS 

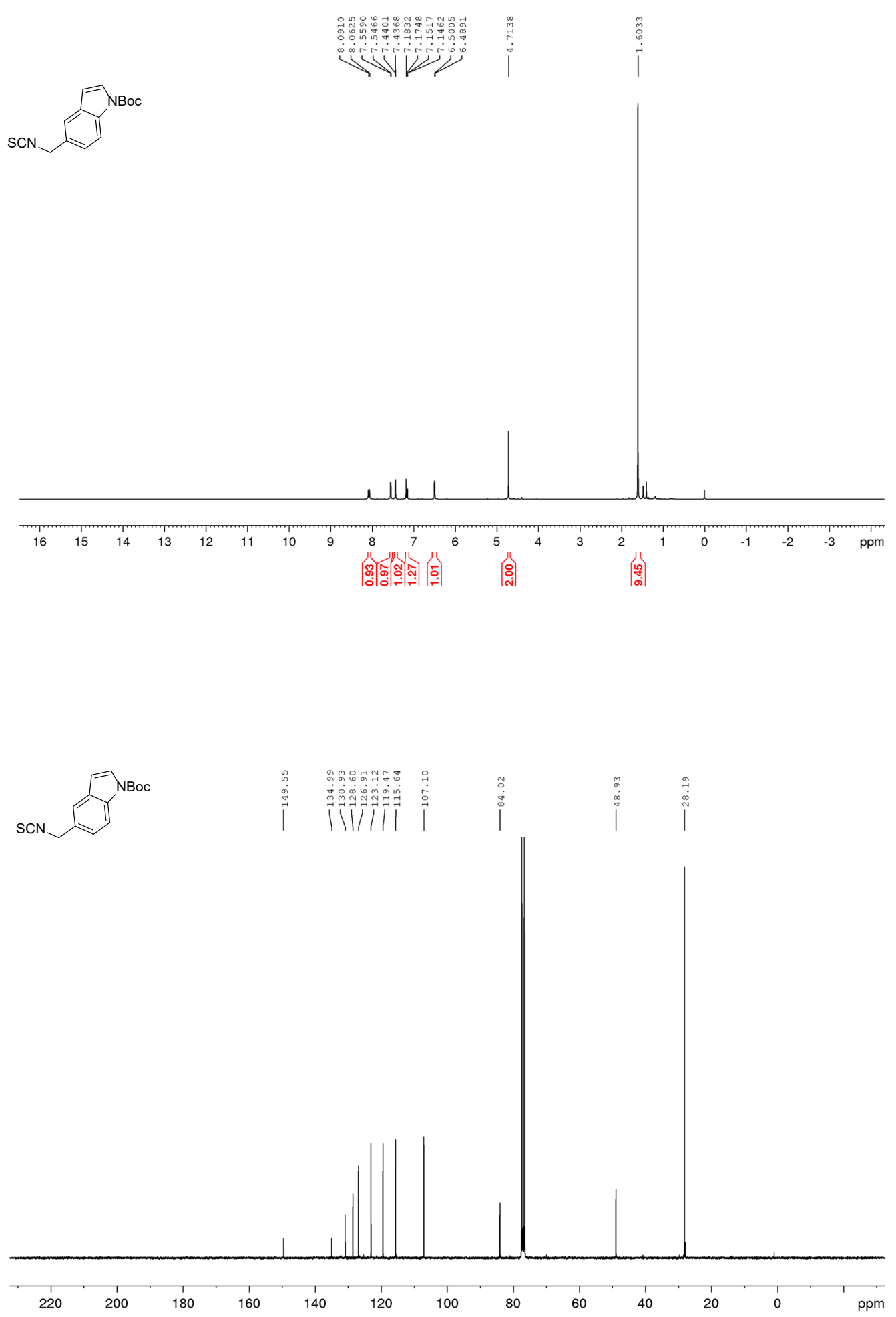

Supplemental Figure 30. ${ }^{1} \mathrm{H}$ NMR and ${ }^{13} \mathrm{C}$ NMR (300 MHz, $\left.75.5 \mathrm{MHz} \mathrm{CD}_{3} \mathrm{OD}\right)$ of IBLCS 


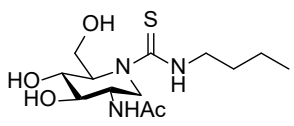
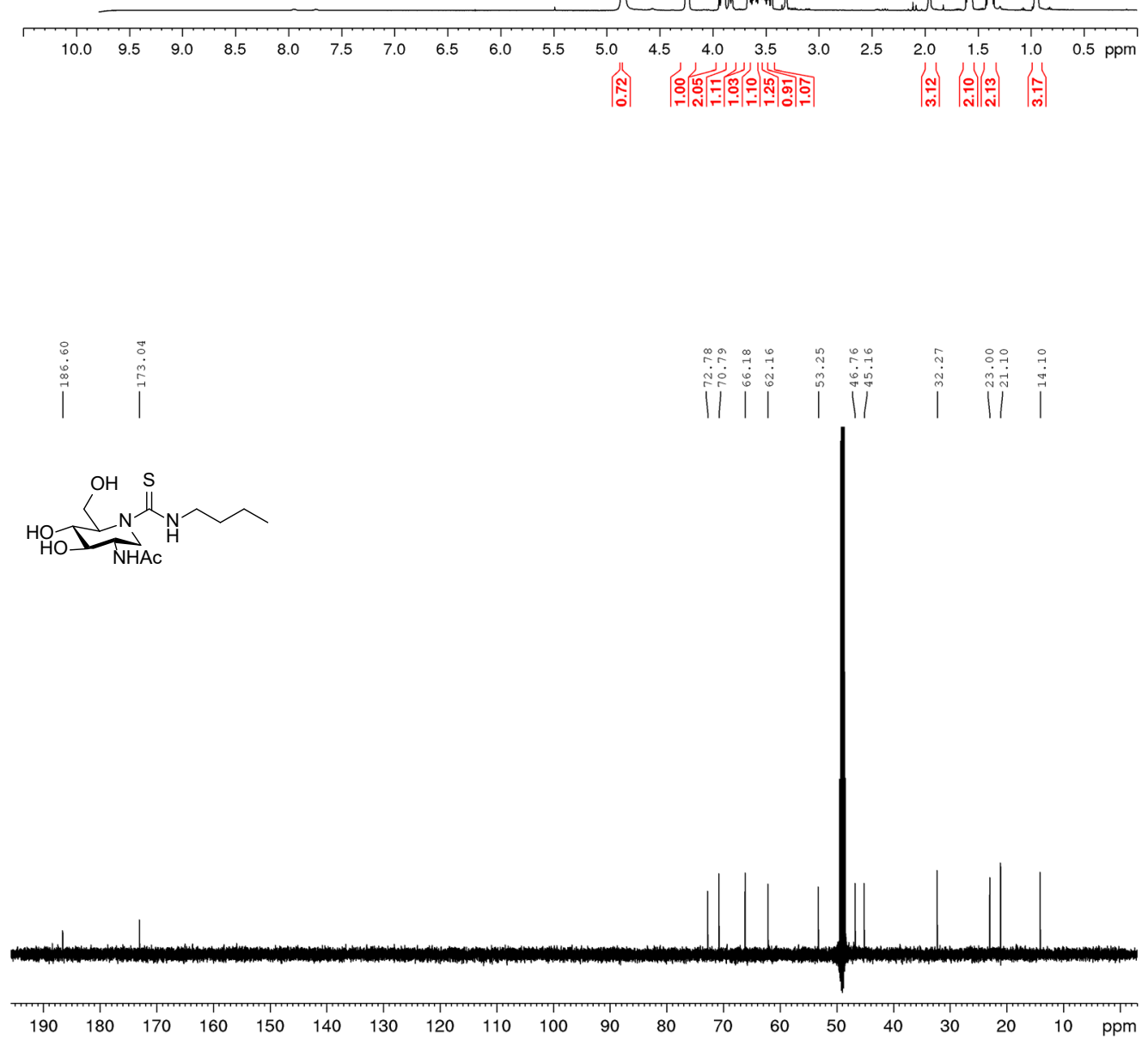

Supplemental Figure 31. ${ }^{1} \mathrm{H}$ NMR and ${ }^{13} \mathrm{C}$ NMR (500 MHz, $\left.125.7 \mathrm{MHz} \mathrm{CD}_{3} \mathrm{OD}\right)$ of 1 

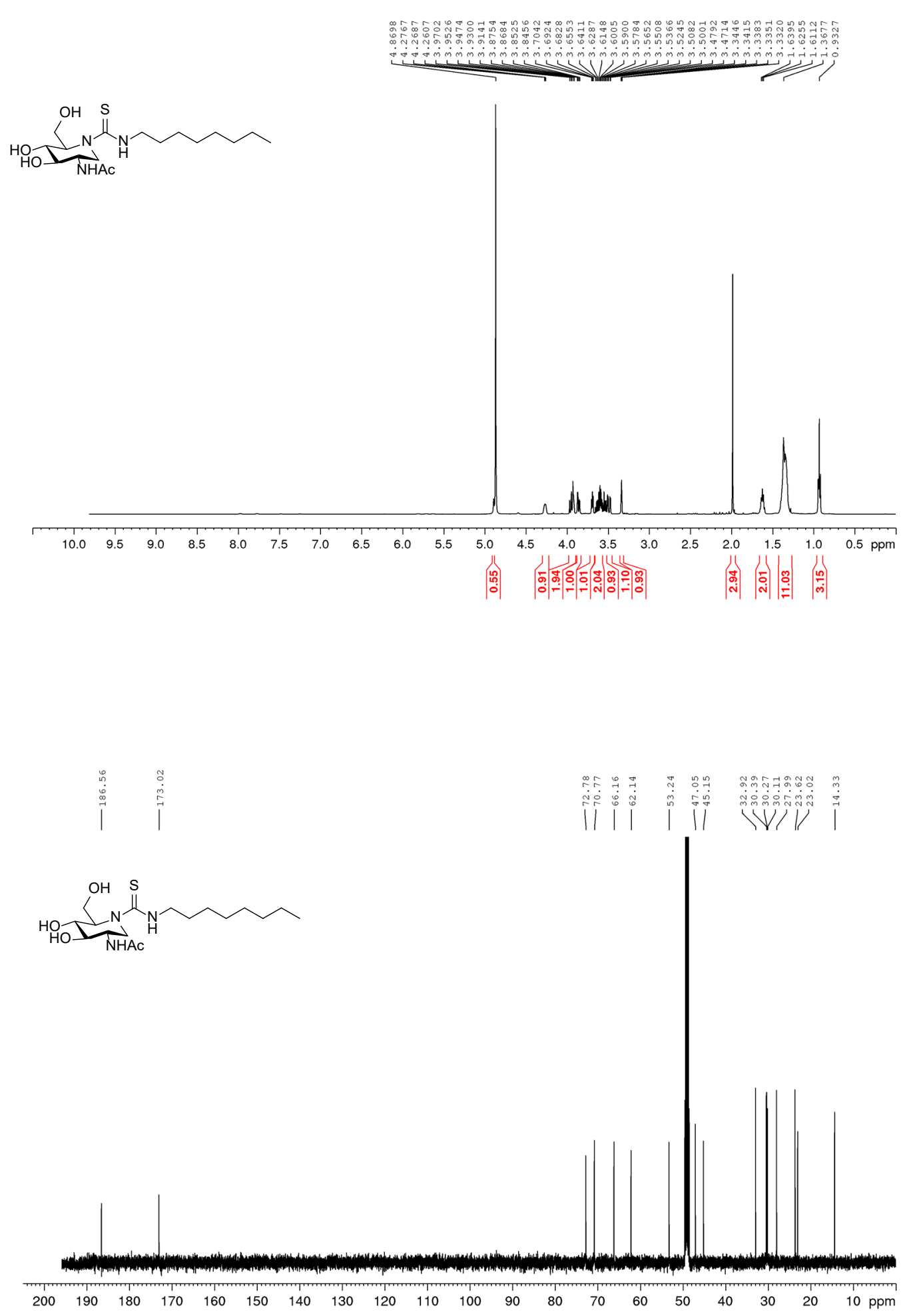

Supplemental Figure 32. ${ }^{1} \mathrm{H}$ NMR and ${ }^{13} \mathrm{C}$ NMR $\left(500 \mathrm{MHz}, 125.7 \mathrm{MHz} \mathrm{CD}_{3} \mathrm{OD}\right)$ of 2 

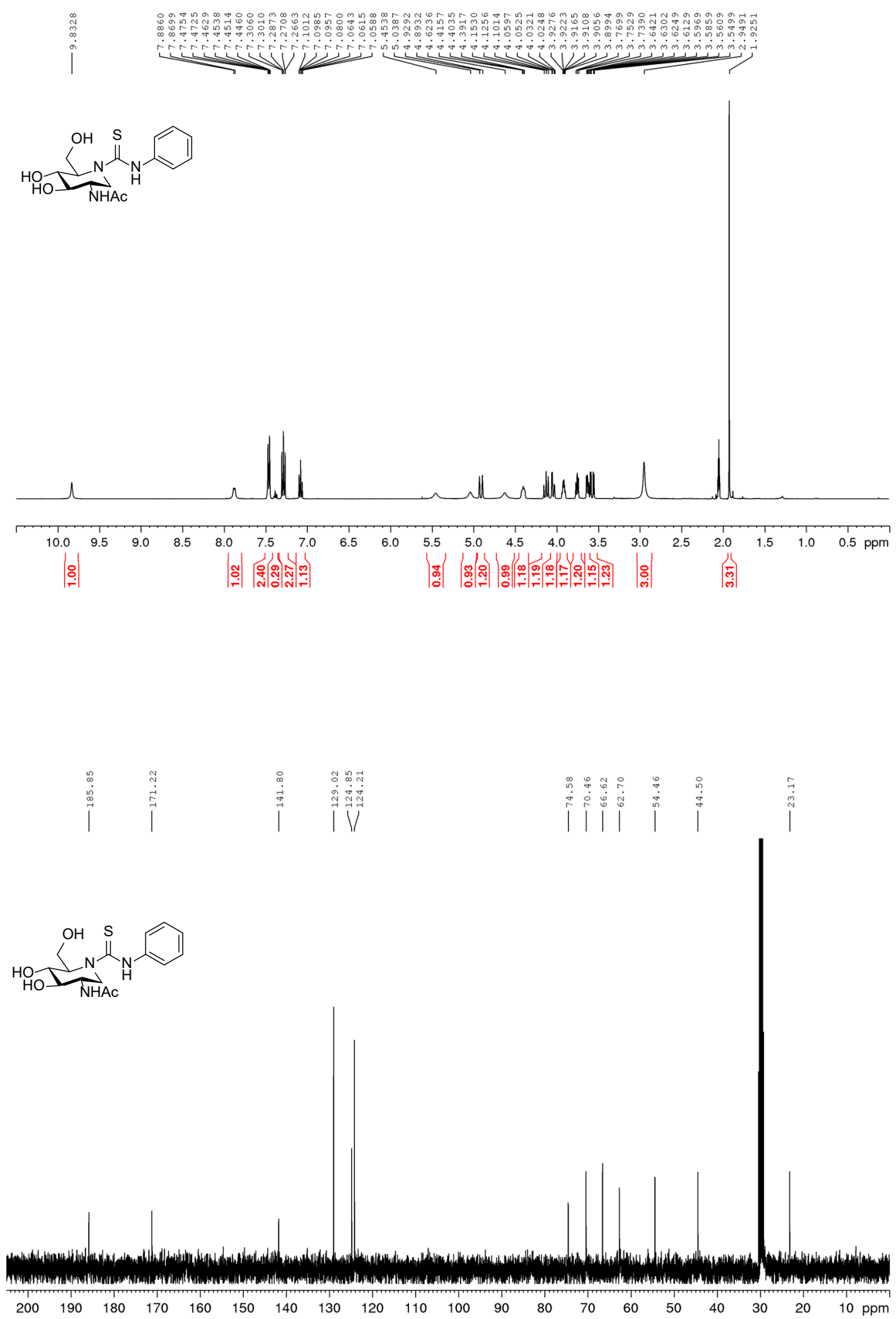

Supplemental Figure 33. ${ }^{1} \mathrm{H}$ NMR and ${ }^{13} \mathrm{C}$ NMR $\left(400 \mathrm{MHz}, 100.6 \mathrm{MHz}\right.$ acetone- $\left.d_{6}\right)$ of 3 

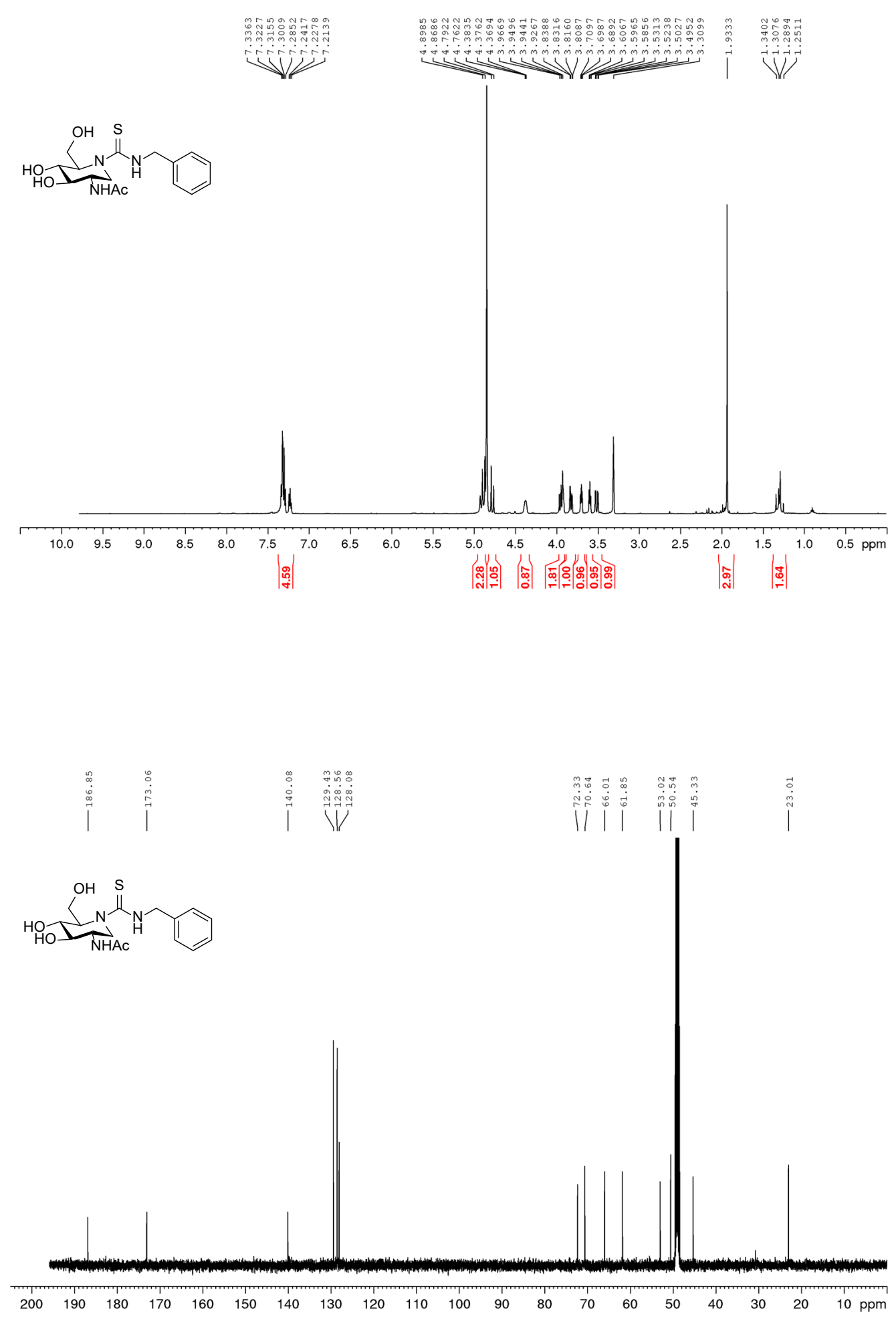

Supplemental Figure 34. ${ }^{1} \mathrm{H}$ NMR and ${ }^{13} \mathrm{C}$ NMR $\left(500 \mathrm{MHz}, 125.7 \mathrm{MHz} \mathrm{CD}_{3} \mathrm{OD}\right)$ of 4 

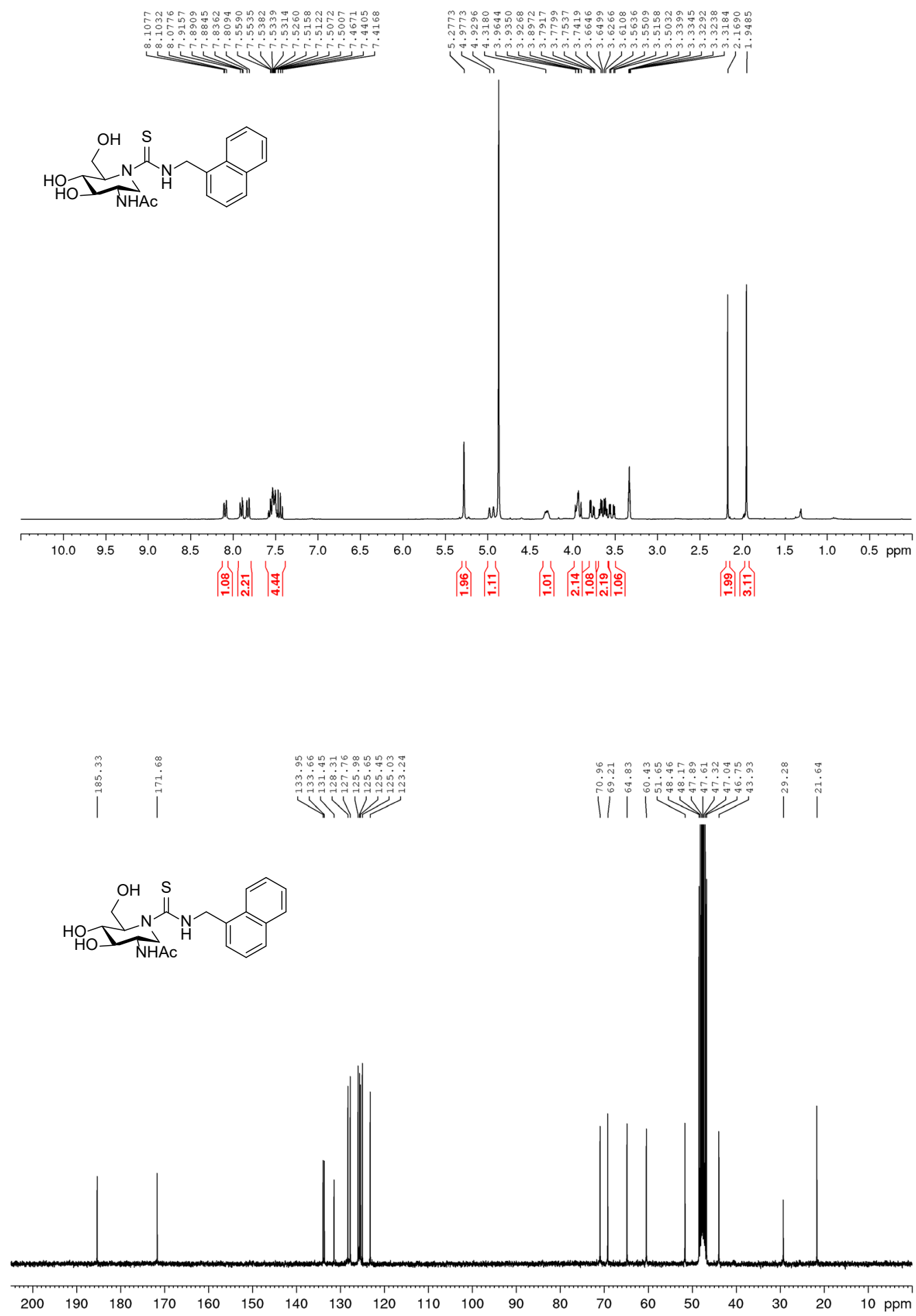

Supplemental Figure 35. ${ }^{1} \mathrm{H}$ NMR and ${ }^{13} \mathrm{C}$ NMR $\left(300 \mathrm{MHz}, 100.6 \mathrm{MHz} \mathrm{CD}_{3} \mathrm{OD}\right)$ of 5 


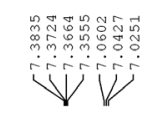

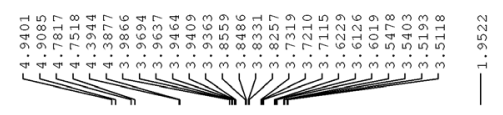

(NHAC)
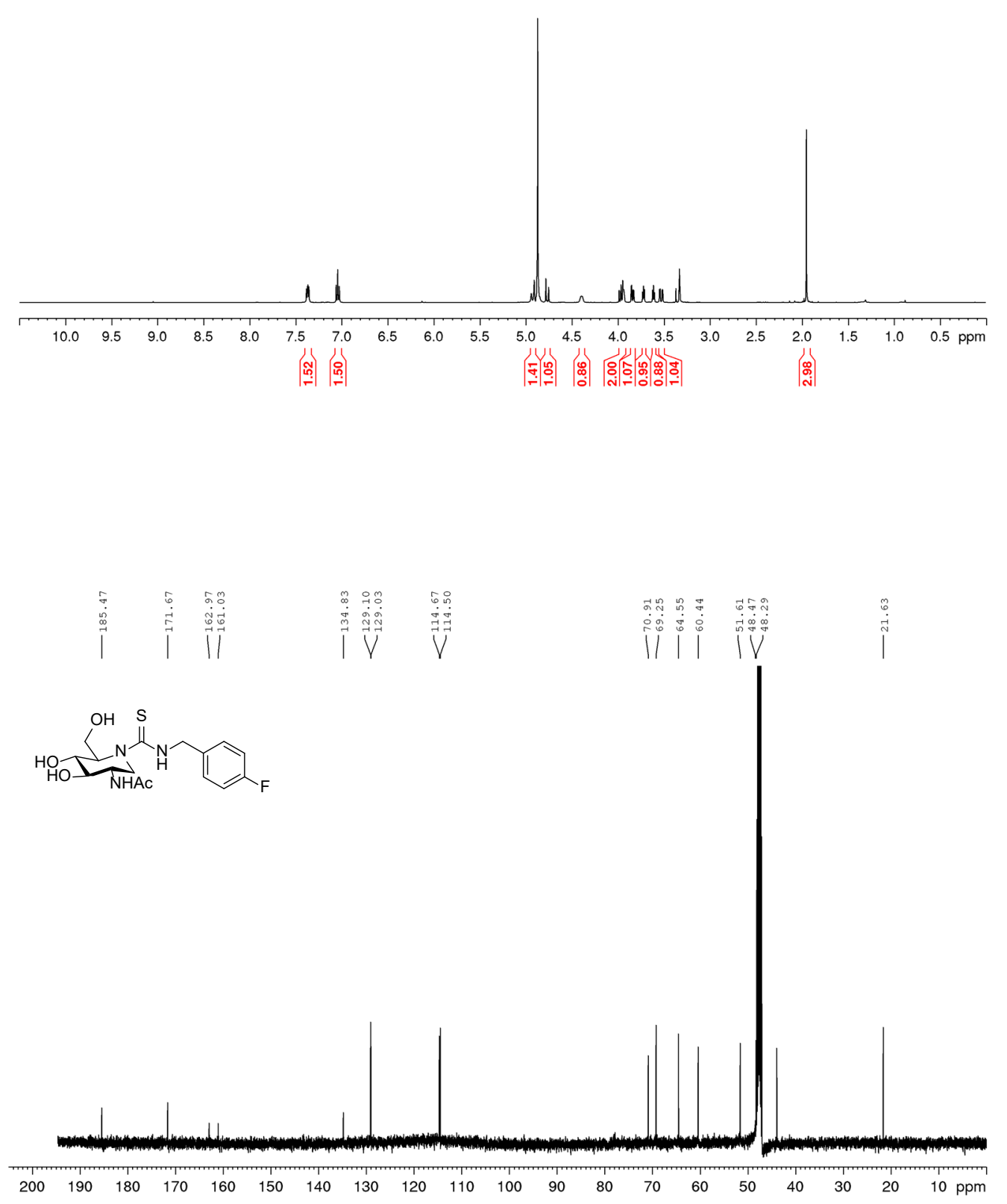

Supplemental Figure 36. ${ }^{1} \mathrm{H} \mathrm{NMR}$ and ${ }^{13} \mathrm{C} \mathrm{NMR}\left(500 \mathrm{MHz}, 125.7 \mathrm{MHz} \mathrm{CD}_{3} \mathrm{OD}\right)$ of 6 


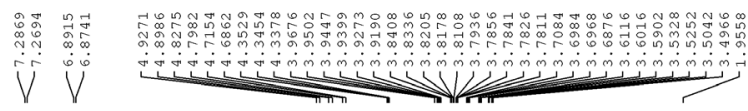

NHAC
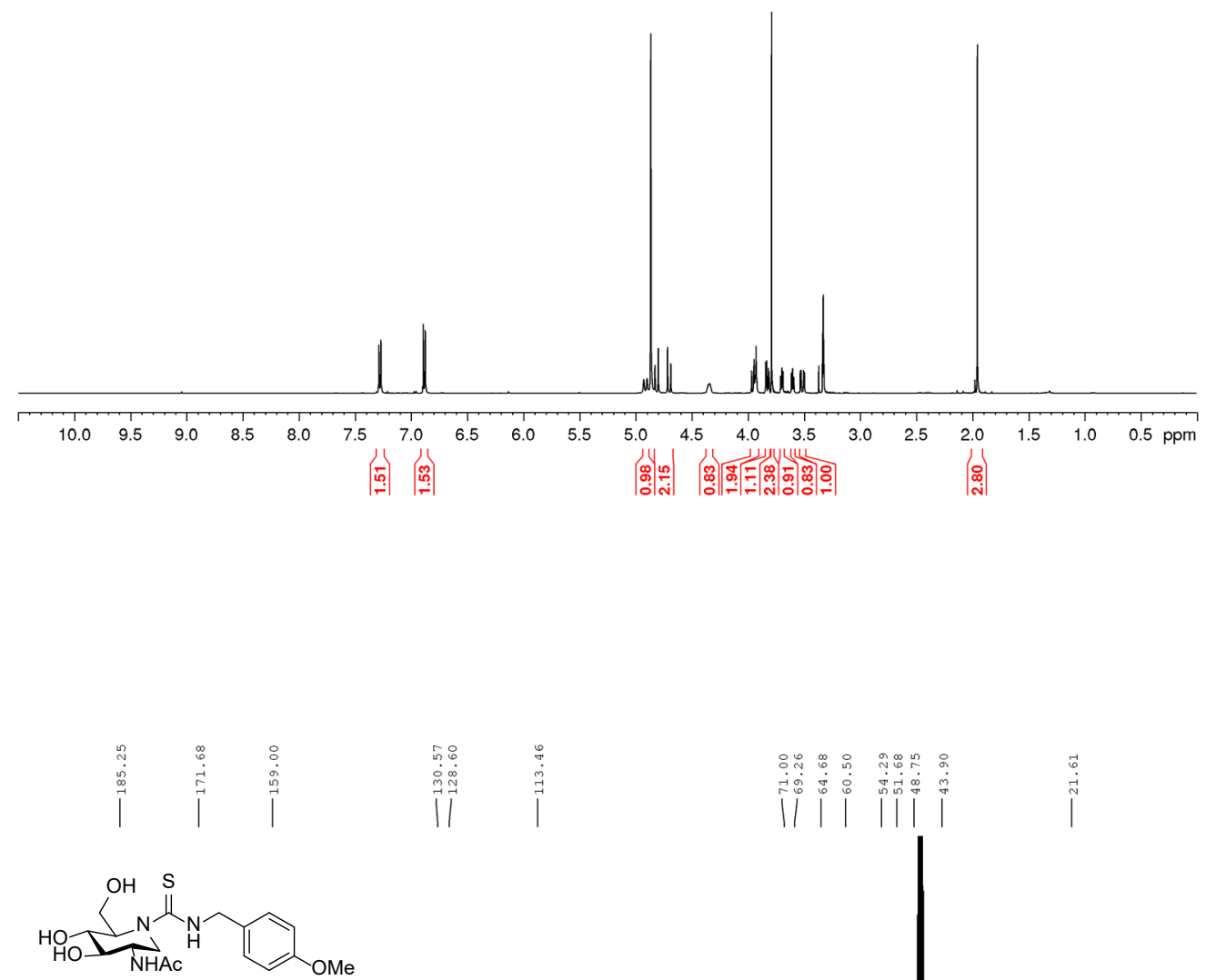

NHAC

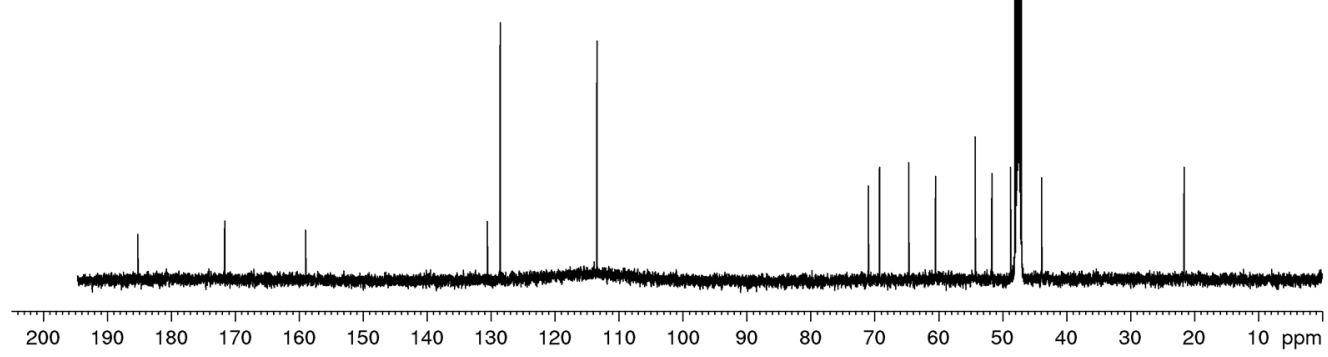

Supplemental Figure 37. ${ }^{1} \mathrm{H}$ NMR and ${ }^{13} \mathrm{C}$ NMR $\left(500 \mathrm{MHz}, 125.7 \mathrm{MHz} \mathrm{CD}_{3} \mathrm{OD}\right)$ of 7 

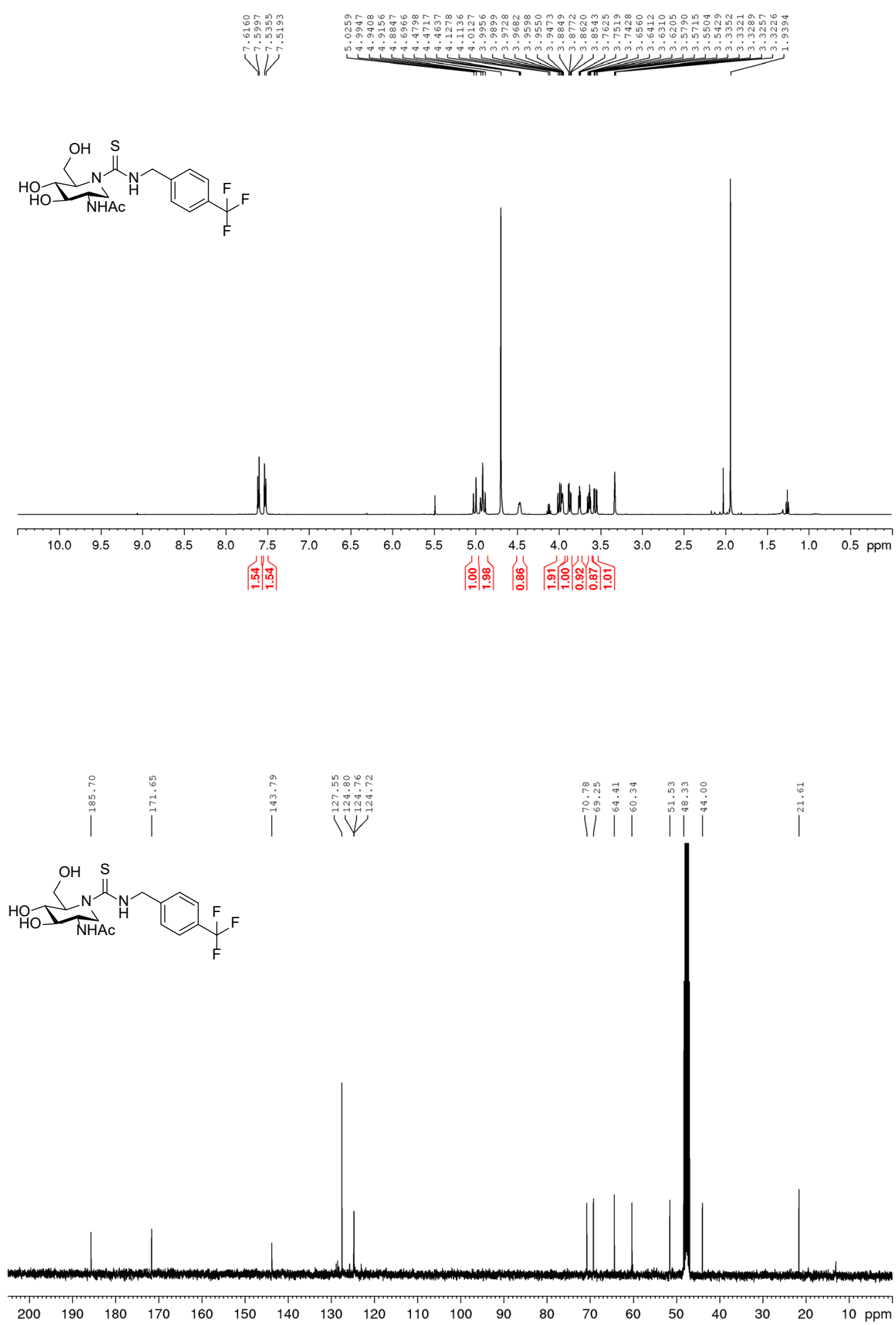

Supplemental Figure 38. ${ }^{1} \mathrm{H}$ NMR and ${ }^{13} \mathrm{C}$ NMR $\left(500 \mathrm{MHz}, 125.7 \mathrm{MHz} \mathrm{CD}_{3} \mathrm{OD}\right)$ of 8 

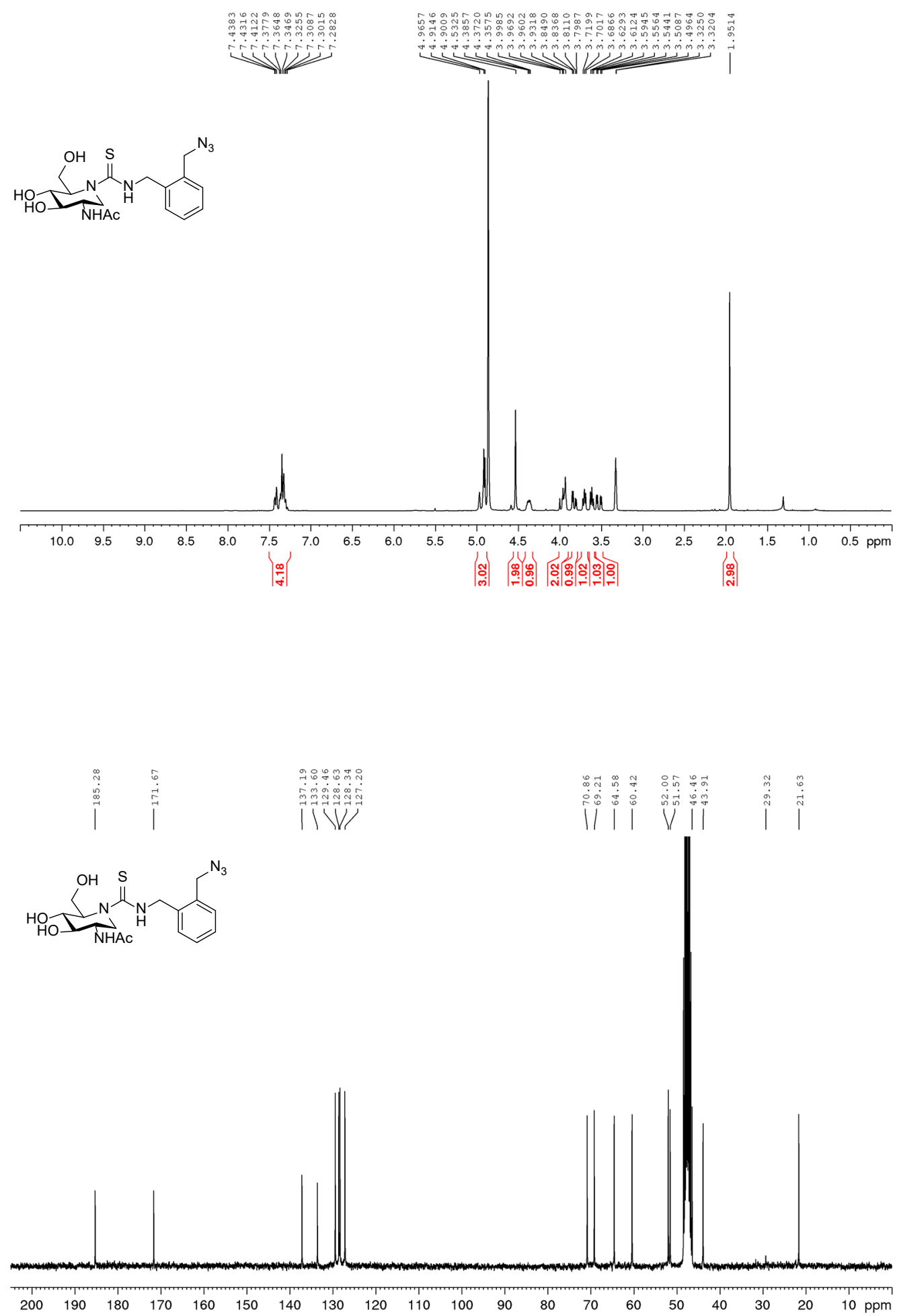

Supplemental Figure 39. ${ }^{1} \mathrm{H}$ NMR and ${ }^{13} \mathrm{C}$ NMR $\left(300 \mathrm{MHz}, 100.6 \mathrm{MHz} \mathrm{CD}_{3} \mathrm{OD}\right)$ of 9 

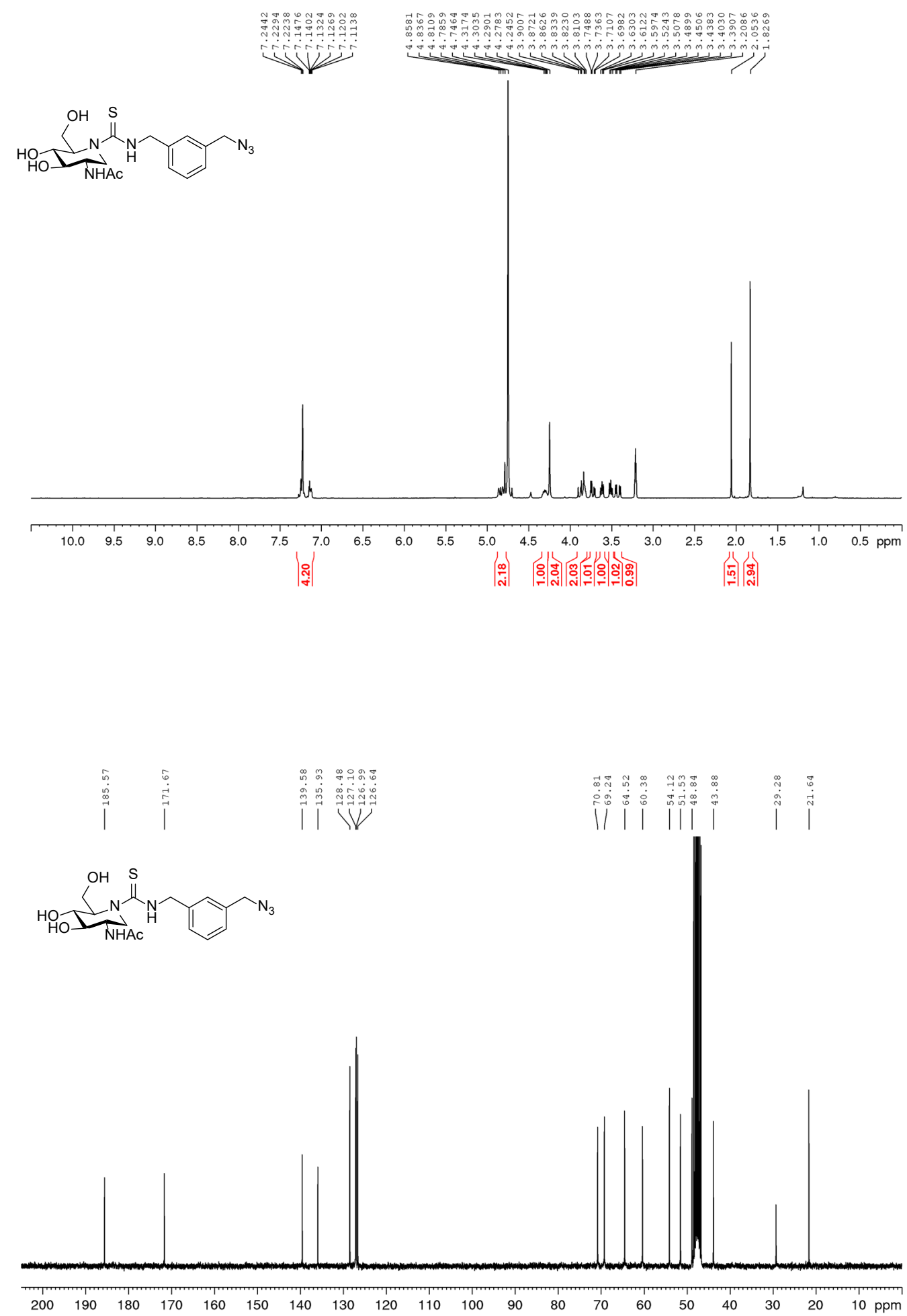

Supplemental Figure 40. ${ }^{1} \mathrm{H}$ NMR and ${ }^{13} \mathrm{C}$ NMR (300 MHz, 100.6 MHz CD $\left.\mathrm{CD}_{3} \mathrm{O}\right)$ of 10 

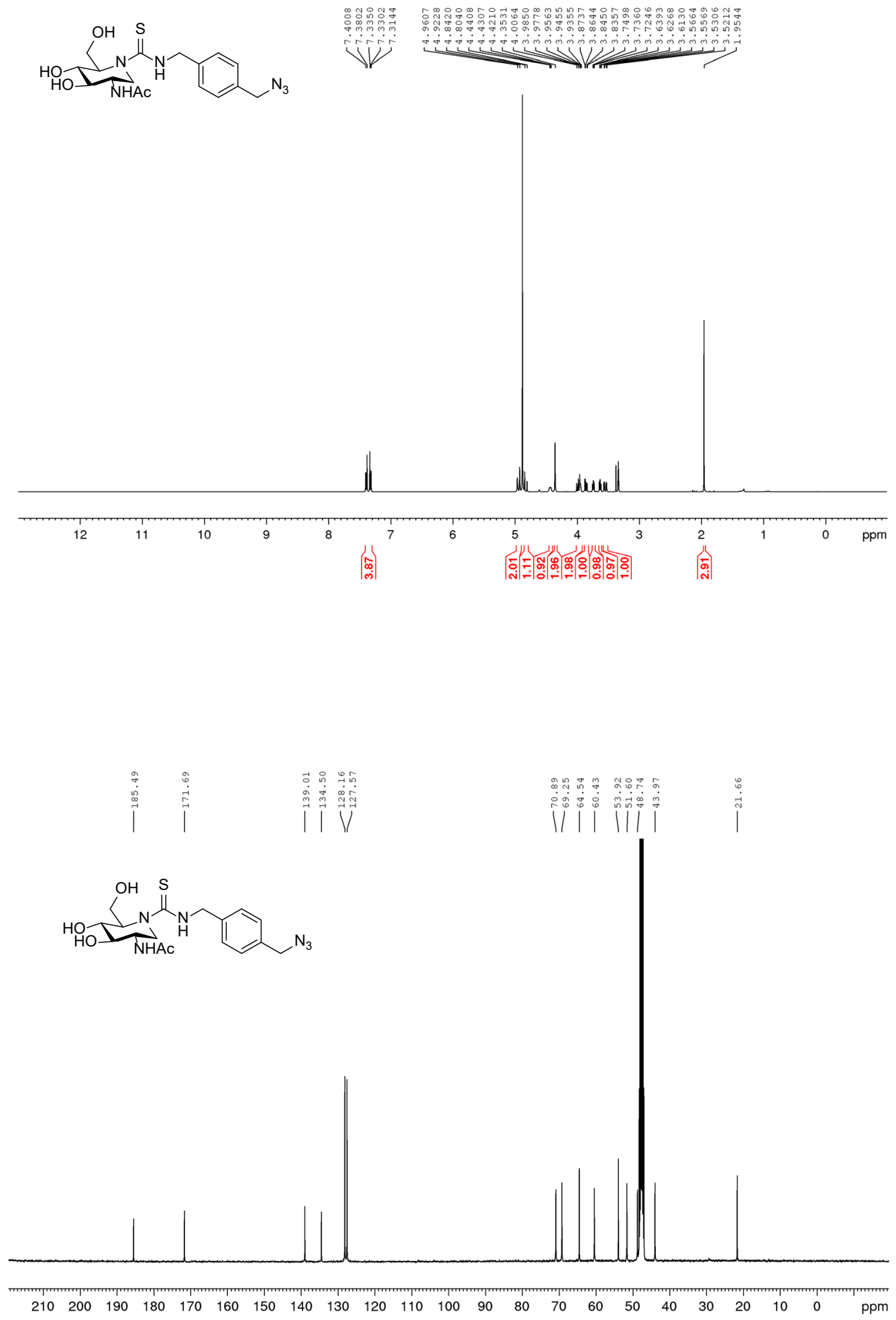

Supplemental Figure 41. ${ }^{1} \mathrm{H}$ NMR and ${ }^{13} \mathrm{C}$ NMR (400 MHz, $100.6 \mathrm{MHz} \mathrm{CD}_{3} \mathrm{OD}$ ) of 11 

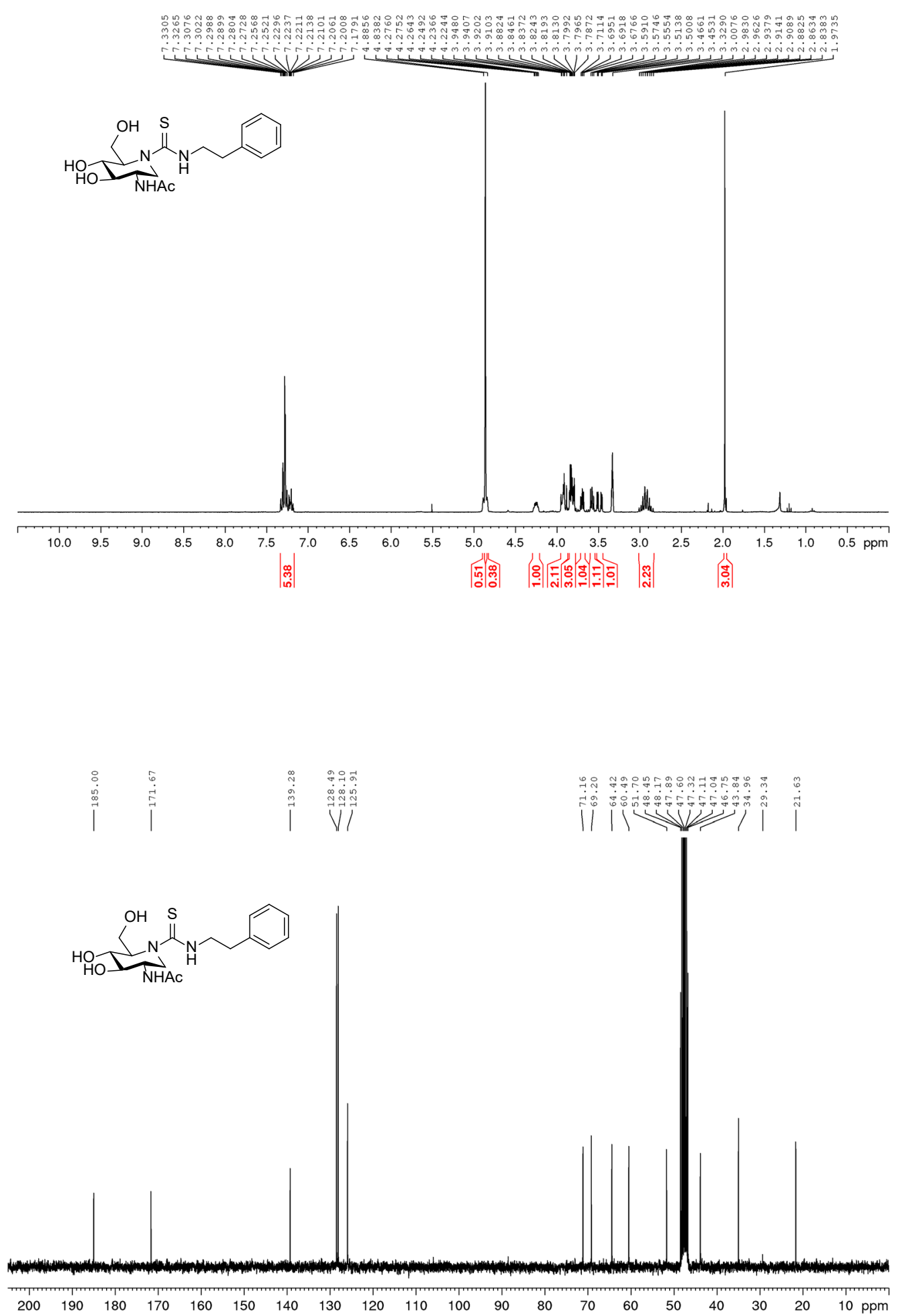

Supplemental Figure $42{ }^{1} \mathrm{H}$ NMR and ${ }^{13} \mathrm{C}$ NMR (300 MHz, $\left.75.5 \mathrm{MHz} \mathrm{CD}_{3} \mathrm{OD}\right)$ of 12 

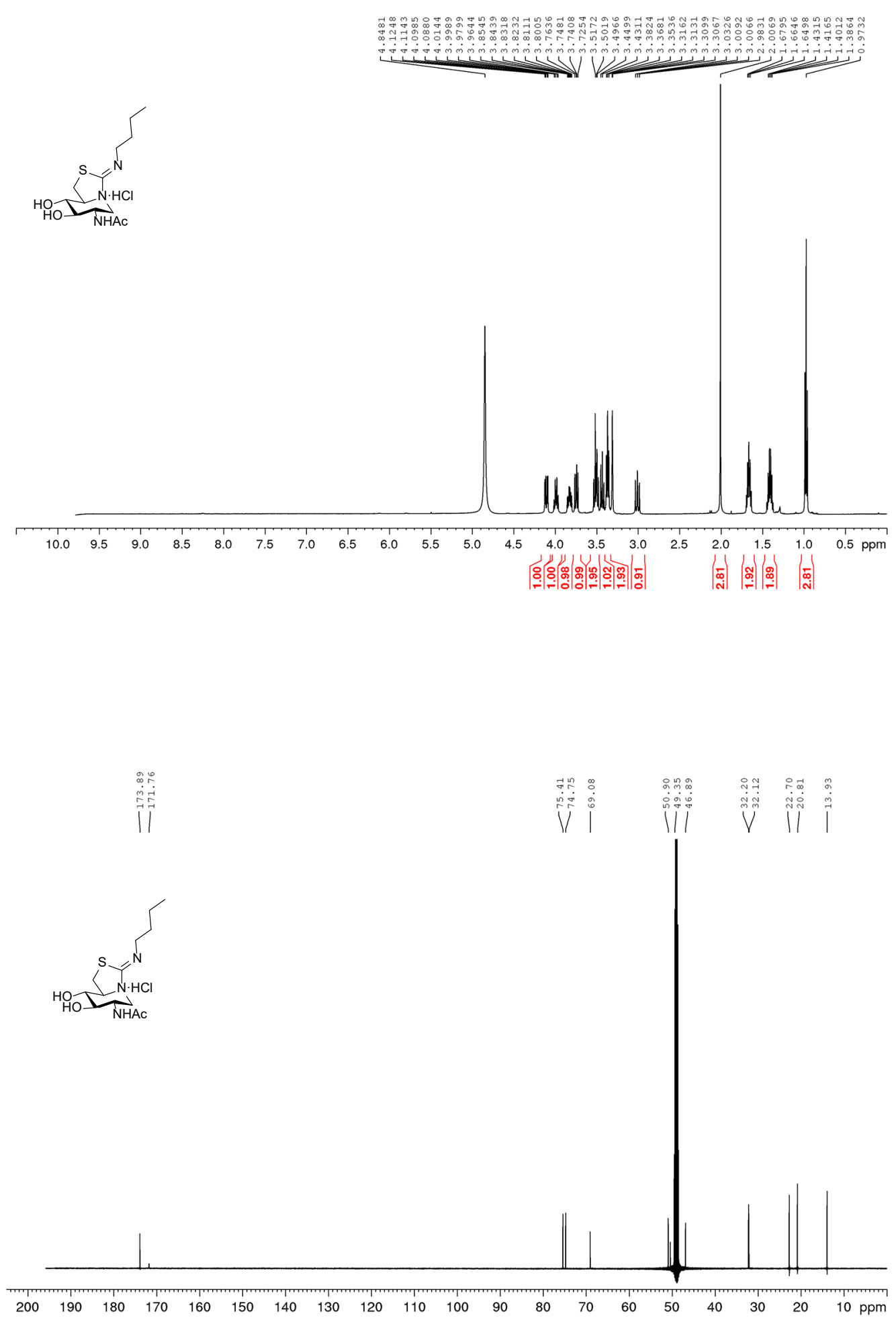

Supplemental Figure 43. ${ }^{1} \mathrm{H}$ NMR and ${ }^{13} \mathrm{C}$ NMR (500 MHz, $\left.125.7 \mathrm{MHz} \mathrm{CD}_{3} \mathrm{OD}\right)$ of 13 

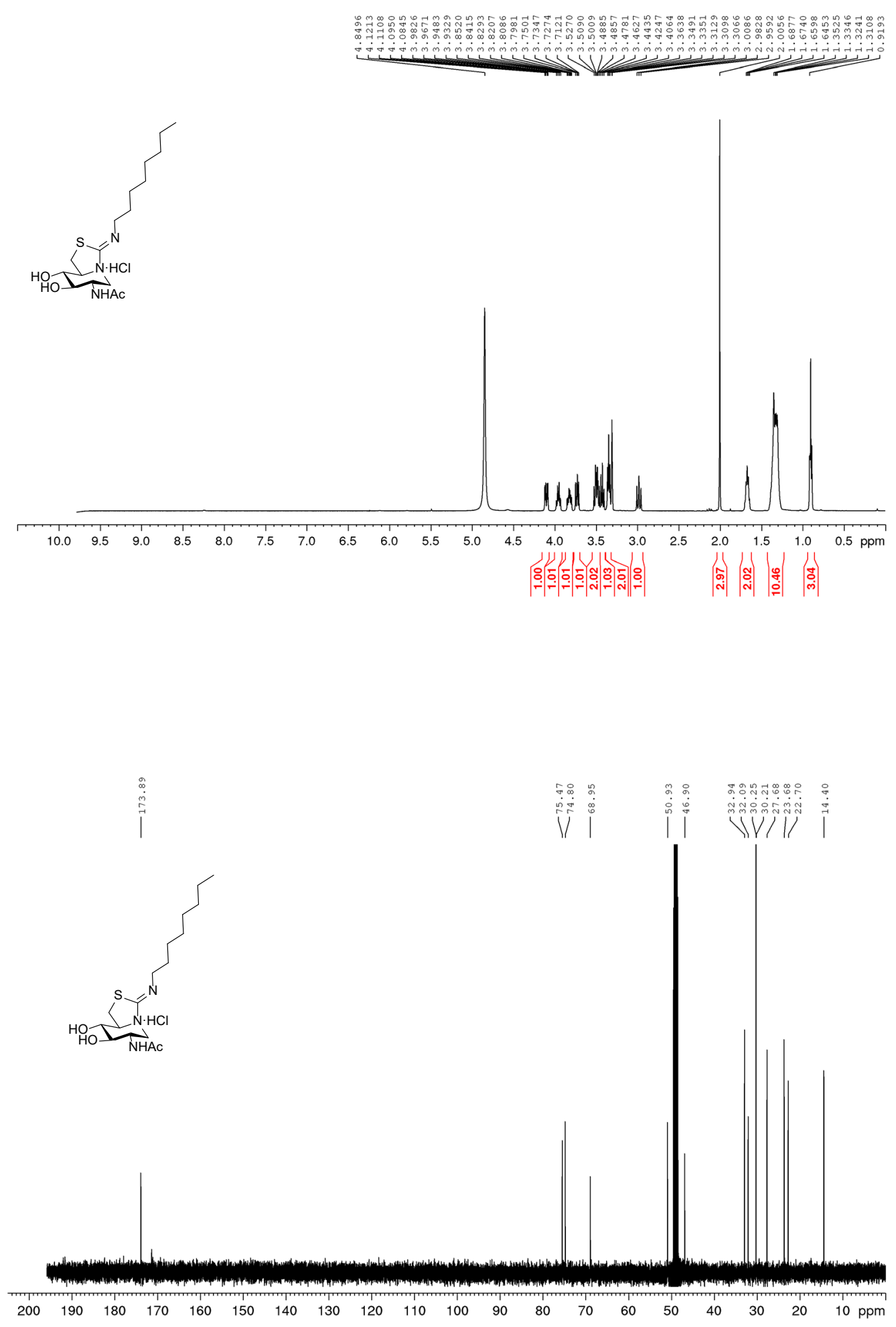

Supplemental Figure 44. ${ }^{1} \mathrm{H}$ NMR and ${ }^{13} \mathrm{C}$ NMR (500 MHz, $\left.125.7 \mathrm{MHz} \mathrm{CD}_{3} \mathrm{OD}\right)$ of 14 

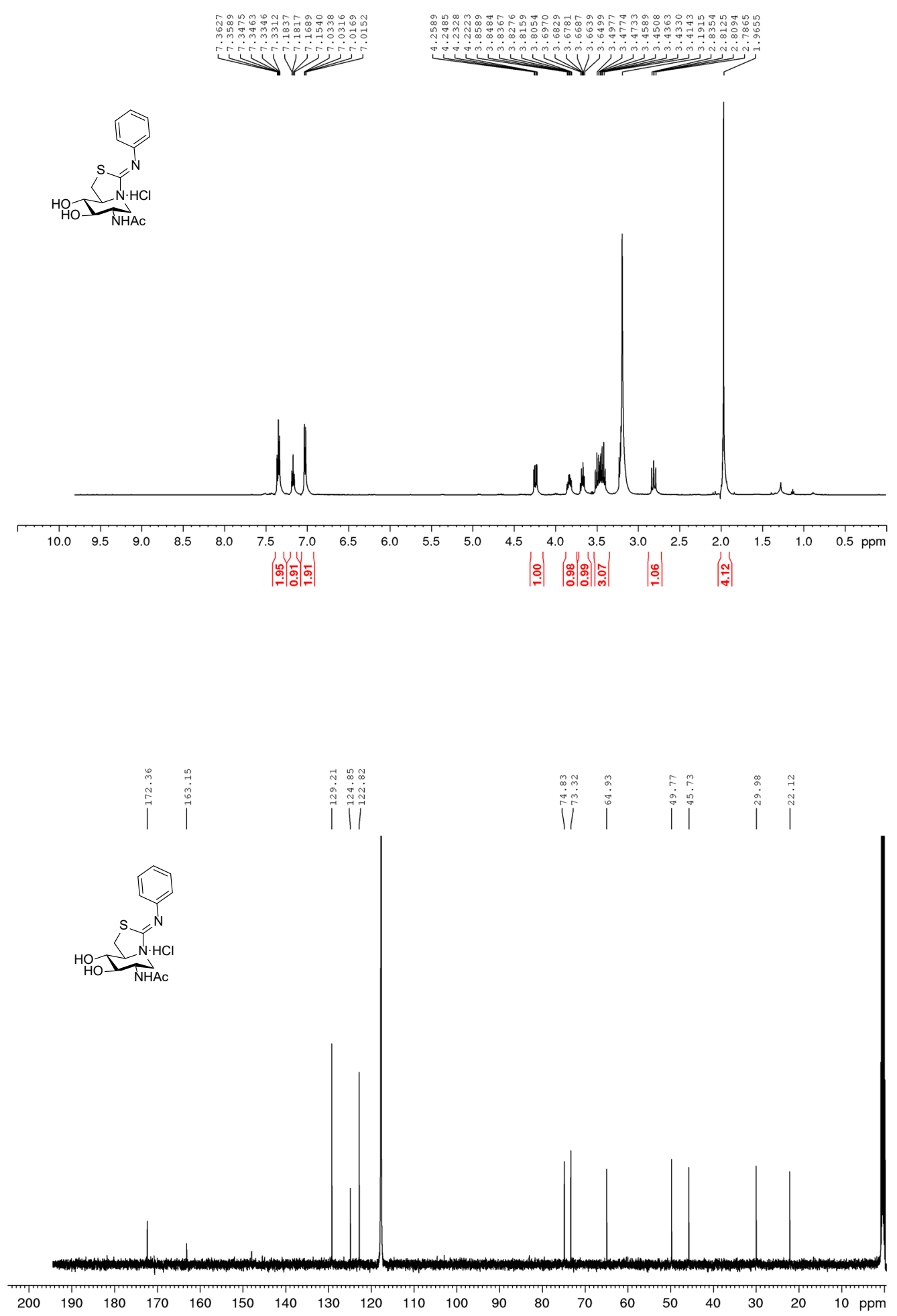

Supplemental Figure 45. ${ }^{1} \mathrm{H}$ NMR and ${ }^{13} \mathrm{C}$ NMR $(500 \mathrm{MHz}, 125.7 \mathrm{MHz}$ 1:10 CD $3 \mathrm{CN}$ $\left.\mathrm{D}_{2} \mathrm{O}\right)$ of $\mathbf{1 5}$ 

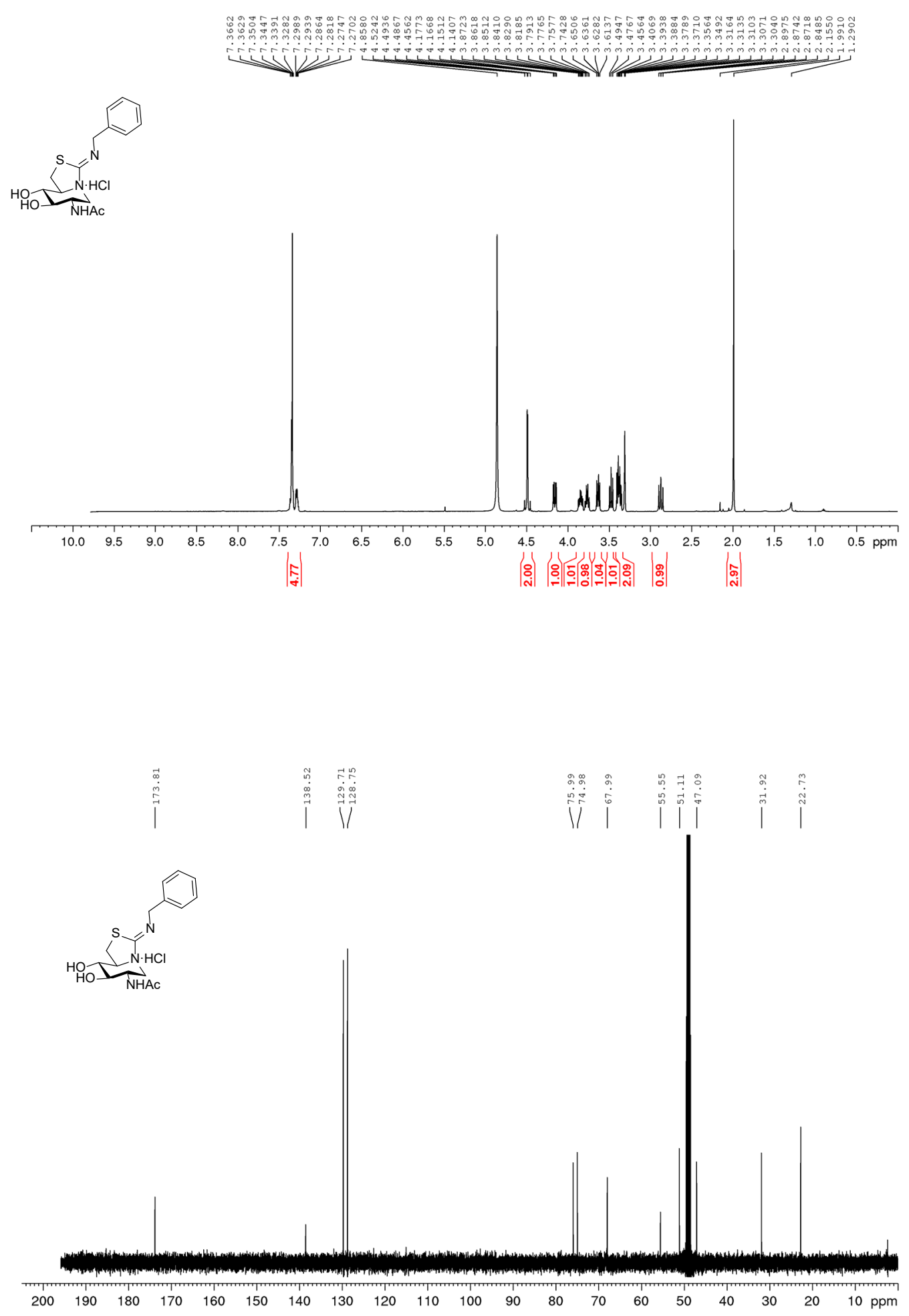

Supplemental Figure 46. ${ }^{1} \mathrm{H}$ NMR and ${ }^{13} \mathrm{C}$ NMR $\left(500 \mathrm{MHz}, 125.7 \mathrm{MHz} \mathrm{CD}_{3} \mathrm{OD}\right)$ of 16 

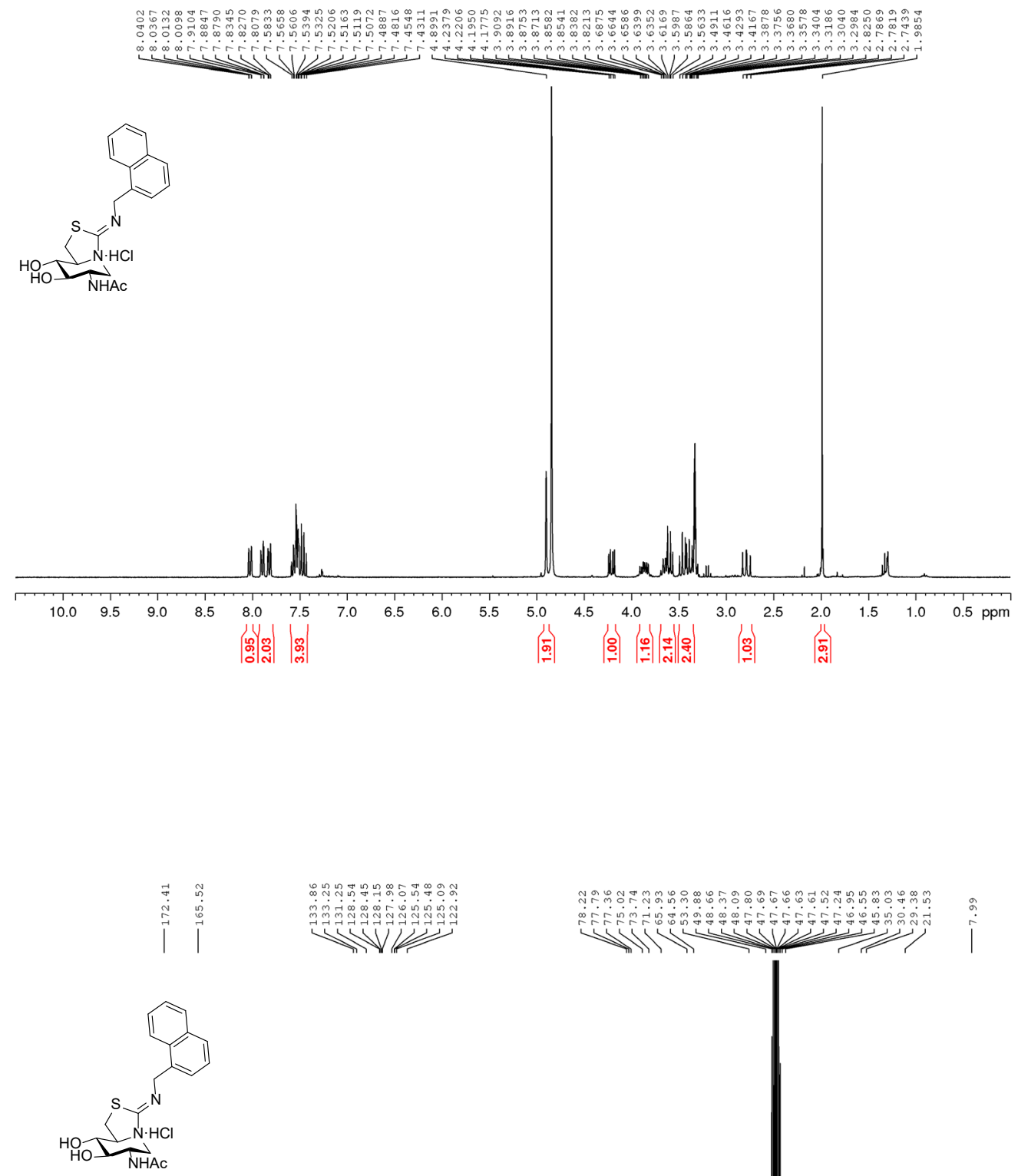

NHAC

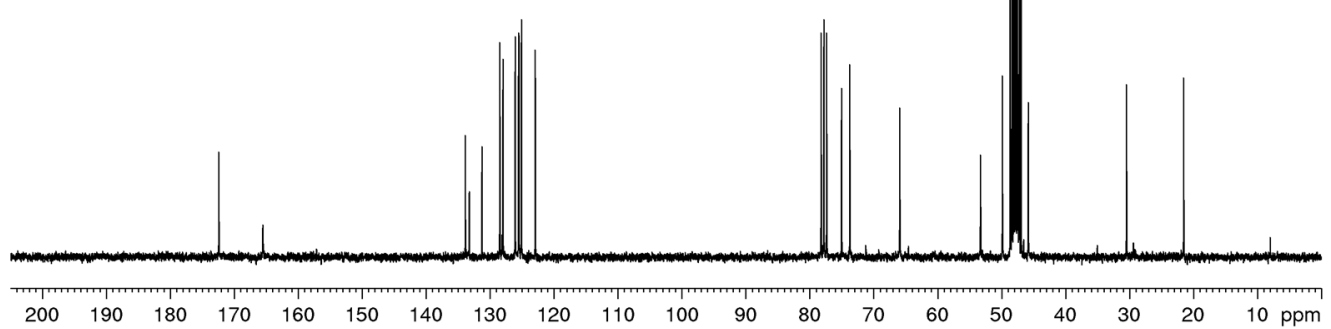

Supplemental Figure 47. ${ }^{1} \mathrm{H}$ NMR and ${ }^{13} \mathrm{C}$ NMR $(300 \mathrm{MHz}, 100.6 \mathrm{MHz}$ 8:1 CD $\mathrm{CD}$ $\left.\mathrm{CDCl}_{3}\right)$ of $\mathbf{1 7}$ 

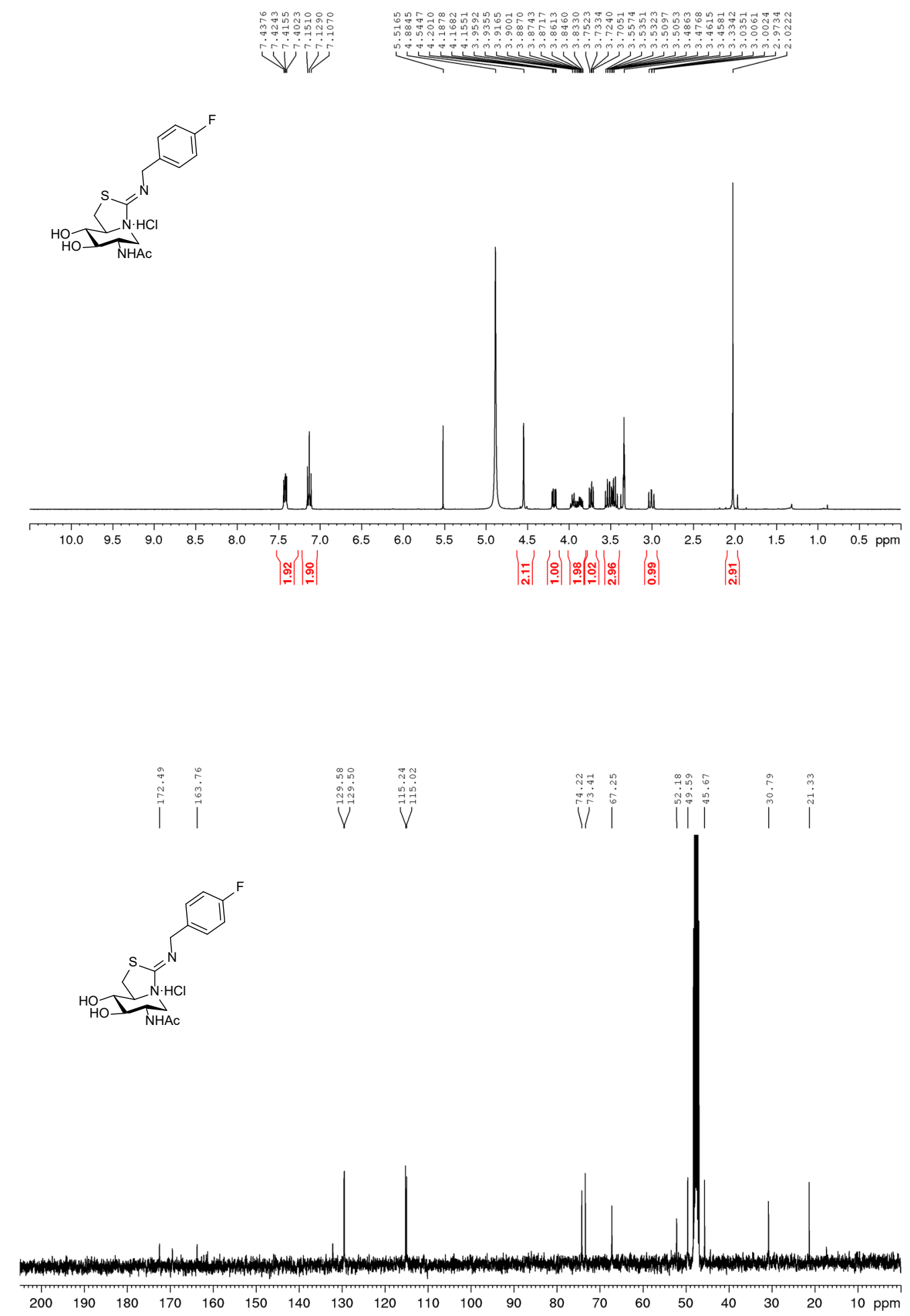

Supplemental Figure 48. ${ }^{1} \mathrm{H}$ NMR and ${ }^{13} \mathrm{C}$ NMR (500 MHz, $\left.125.7 \mathrm{MHz} \mathrm{CD}_{3} \mathrm{OD}\right)$ of 18 

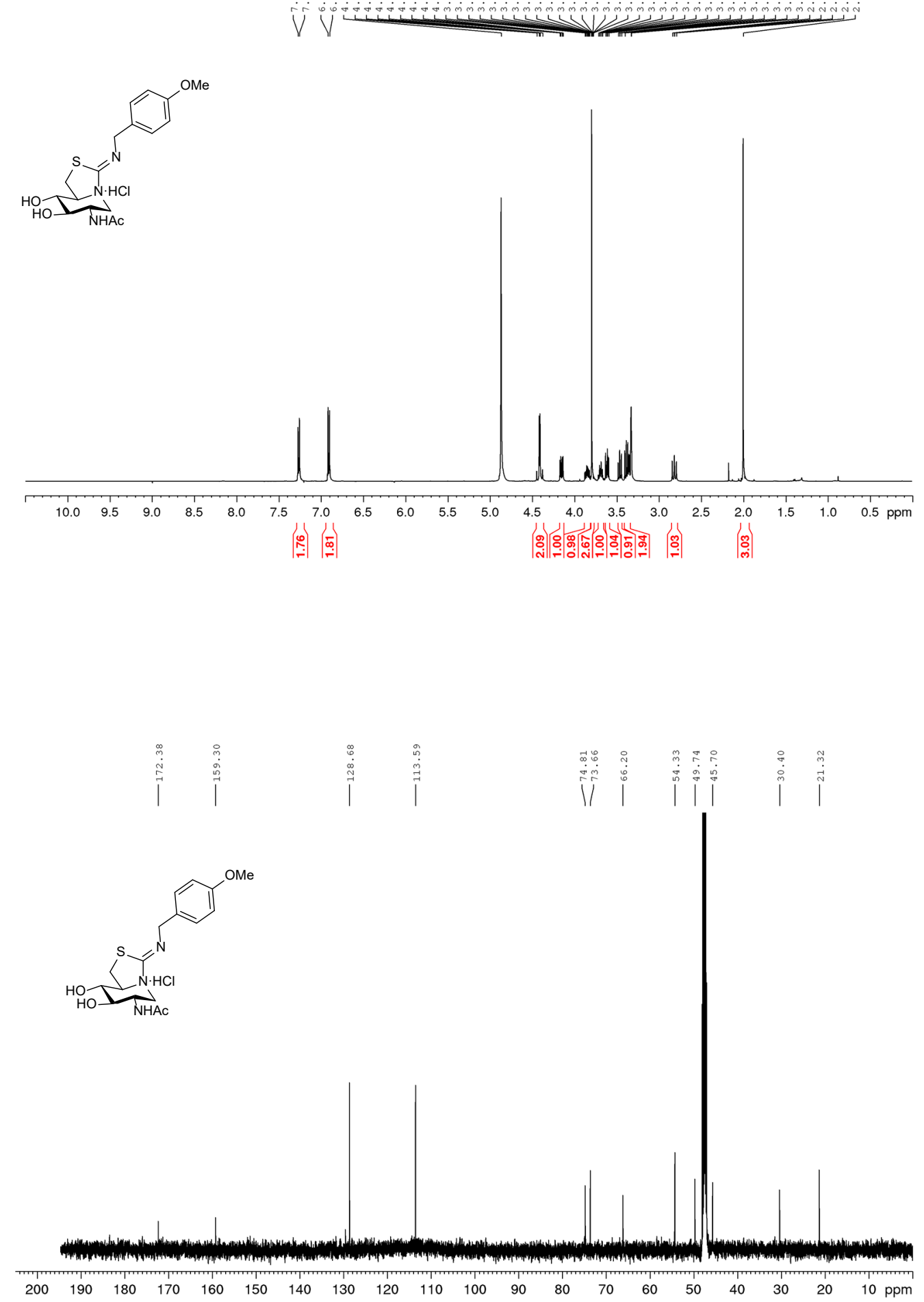

Supplemental Figure 49. ${ }^{1} \mathrm{H}$ NMR and ${ }^{13} \mathrm{C}$ NMR (500 MHz, $\left.125.7 \mathrm{MHz} \mathrm{CD}_{3} \mathrm{OD}\right)$ of 19 

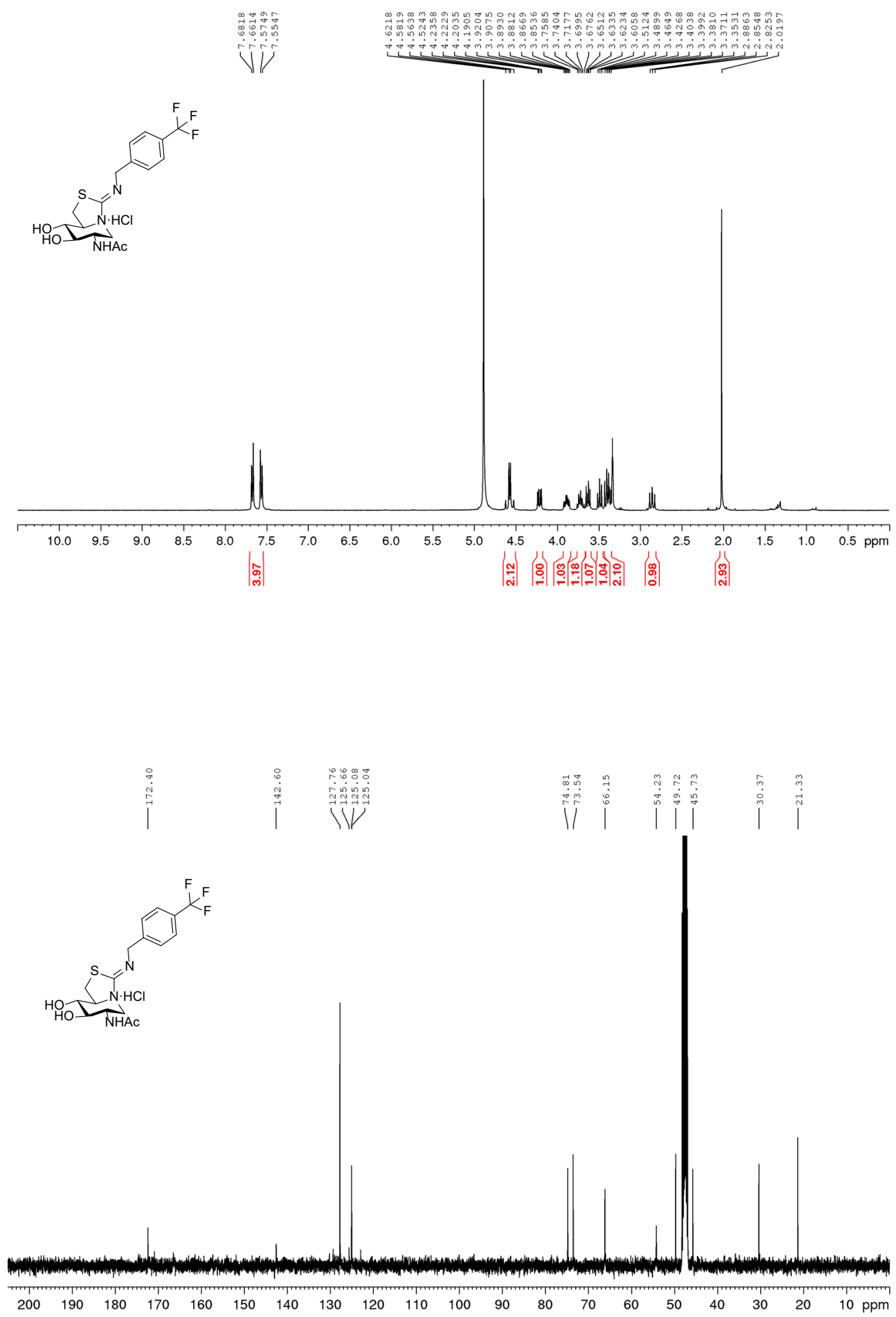

Supplemental Figure 50. ${ }^{1} \mathrm{H}$ NMR and ${ }^{13} \mathrm{C}$ NMR (500 MHz, $\left.125.7 \mathrm{MHz} \mathrm{CD}_{3} \mathrm{OD}\right)$ of 20 

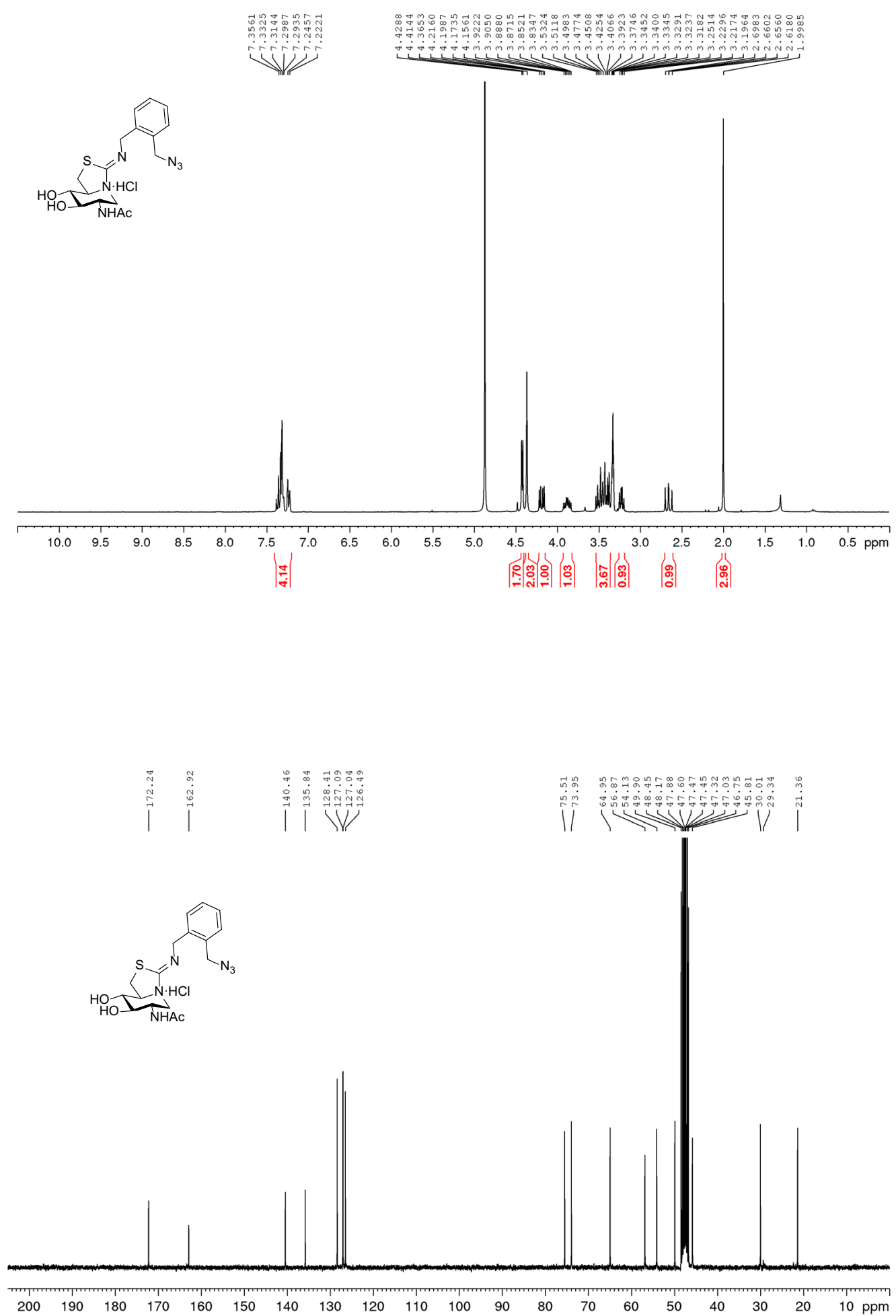

Supplemental Figure 51. ${ }^{1} \mathrm{H}$ NMR and ${ }^{13} \mathrm{C}$ NMR (300 MHz, $\left.75.5 \mathrm{MHz} \mathrm{CD}_{3} \mathrm{OD}\right)$ of 21 

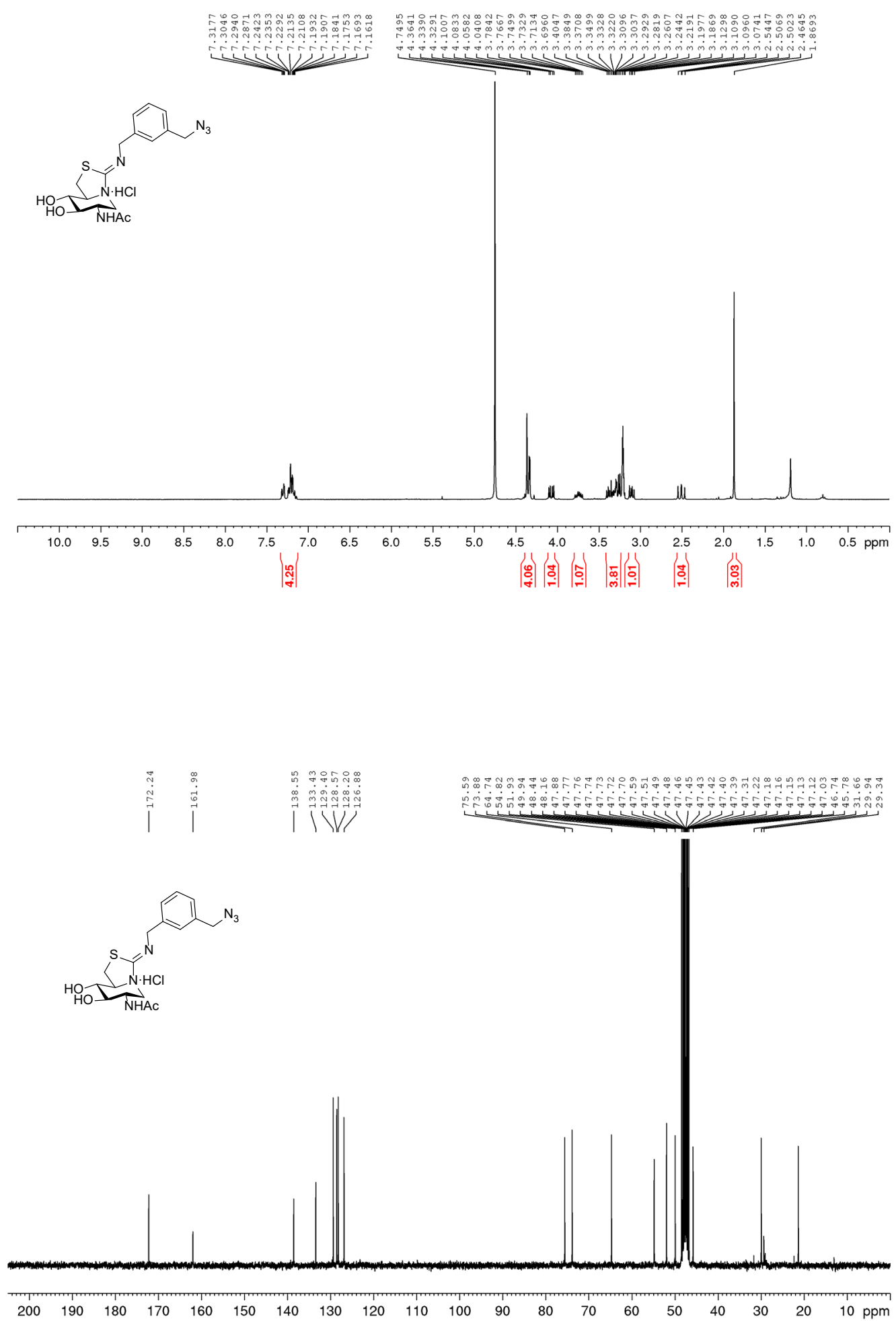

Supplemental Figure 52. ${ }^{1} \mathrm{H}$ NMR and ${ }^{13} \mathrm{C}$ NMR $\left(300 \mathrm{MHz}, 75.5 \mathrm{MHz} \mathrm{CD}_{3} \mathrm{OD}\right)$ of 22 

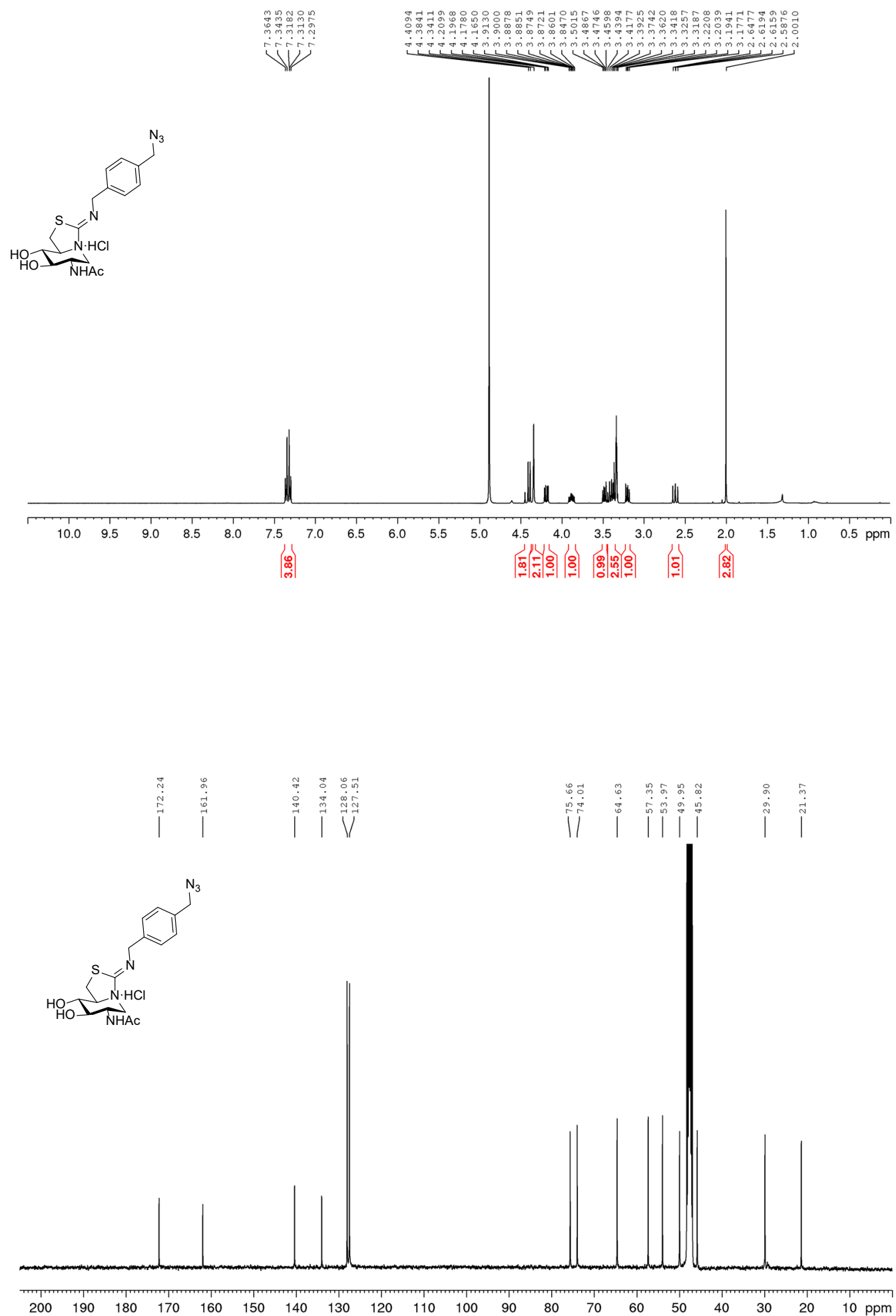

Supplemental Figure 53. ${ }^{1} \mathrm{H}$ NMR and ${ }^{13} \mathrm{C}$ NMR $\left(400 \mathrm{MHz}, 100.6 \mathrm{MHz} \mathrm{CD}_{3} \mathrm{OD}\right)$ of 23 

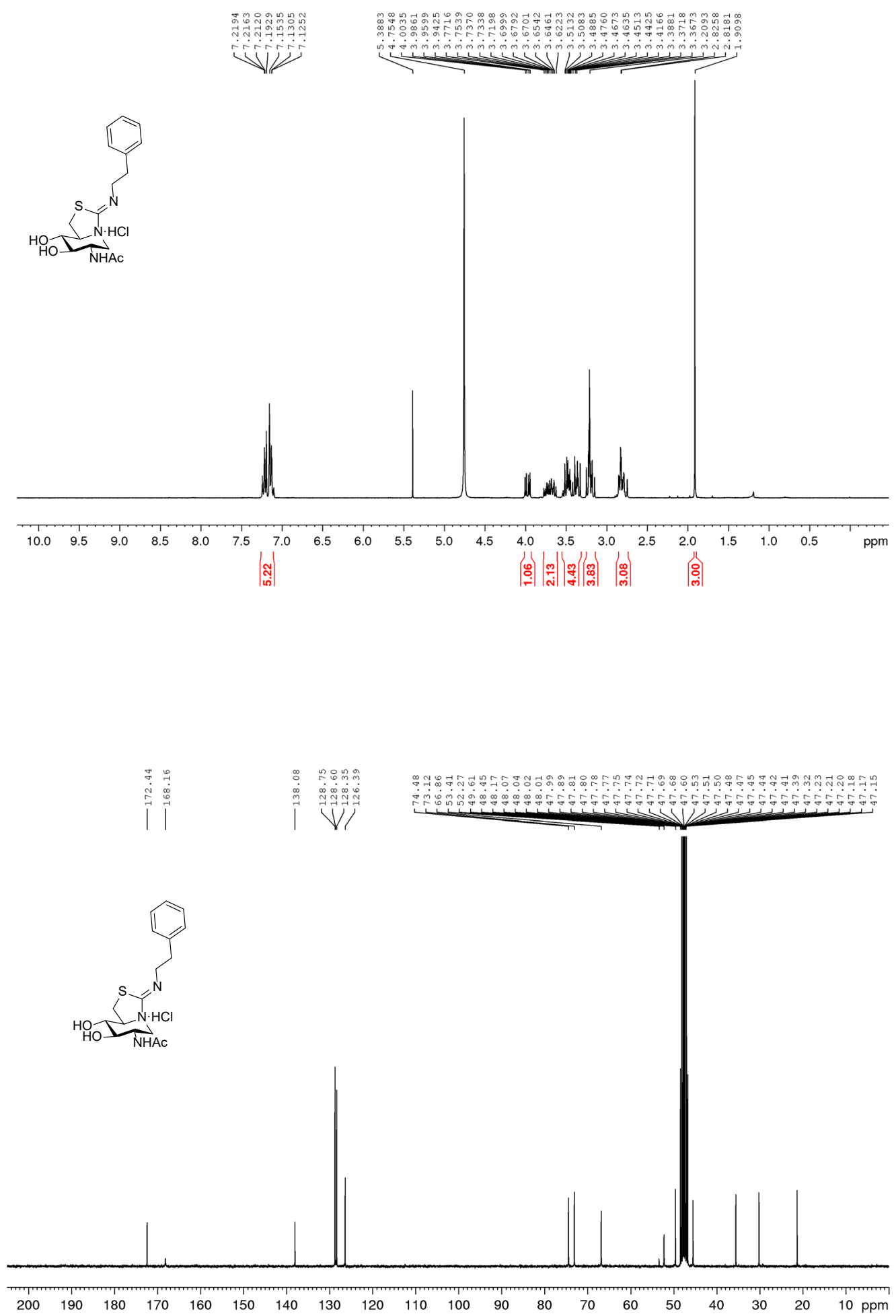

Supplemental Figure 54. ${ }^{1} \mathrm{H}$ NMR and ${ }^{13} \mathrm{C}$ NMR (300 MHz, 75.5 MHz CD 3 OD) of 24 


$$
\sum_{\mathrm{NHBOC}}^{\mathrm{OH}}
$$
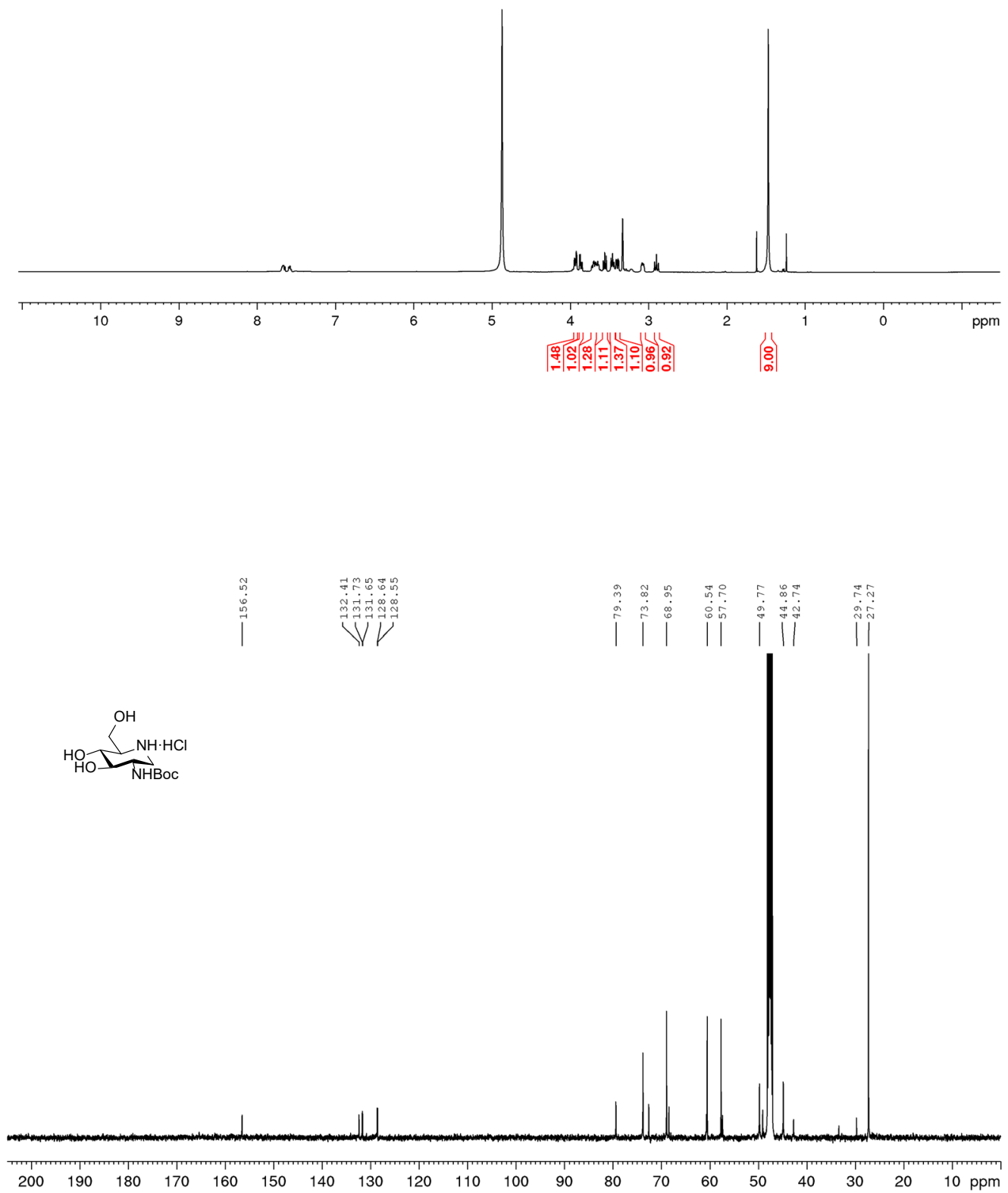

Supplemental Figure 55. ${ }^{1} \mathrm{H}$ NMR and ${ }^{13} \mathrm{C}$ NMR $\left(500 \mathrm{MHz}, 125.7 \mathrm{MHz} \mathrm{CD}_{3} \mathrm{OD}\right)$ of 26 

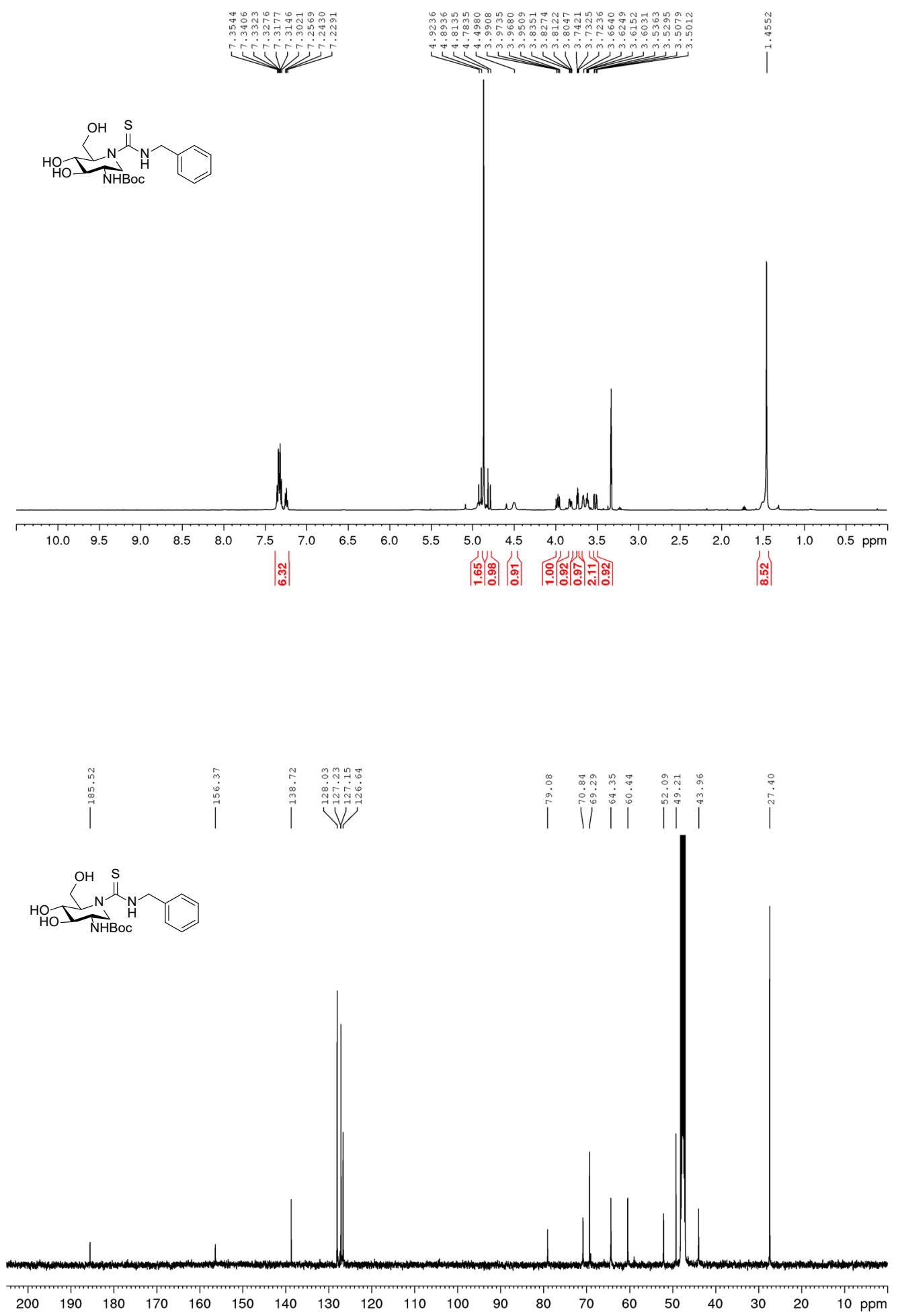

Supplemental Figure 56. ${ }^{1} \mathrm{H}$ NMR and ${ }^{13} \mathrm{C}$ NMR (600 MHz, $\left.150 \mathrm{MHz} \mathrm{CD}_{3} \mathrm{OD}\right)$ of 27 

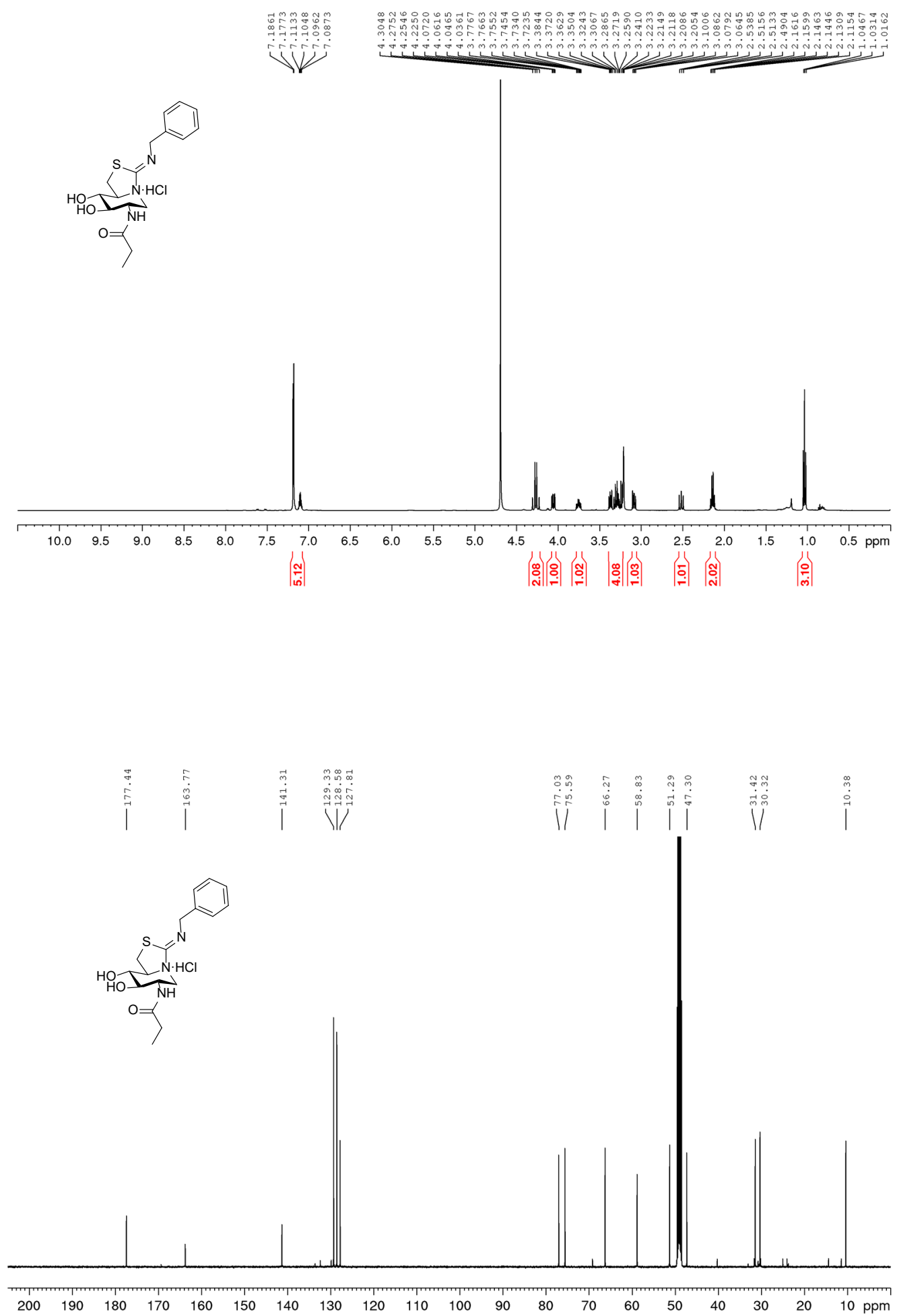

Supplemental Figure 57. ${ }^{1} \mathrm{H}$ NMR and ${ }^{13} \mathrm{C}$ NMR $\left(500 \mathrm{MHz}, 125.7 \mathrm{MHz} \mathrm{CD}_{3} \mathrm{OD}\right)$ of 28 

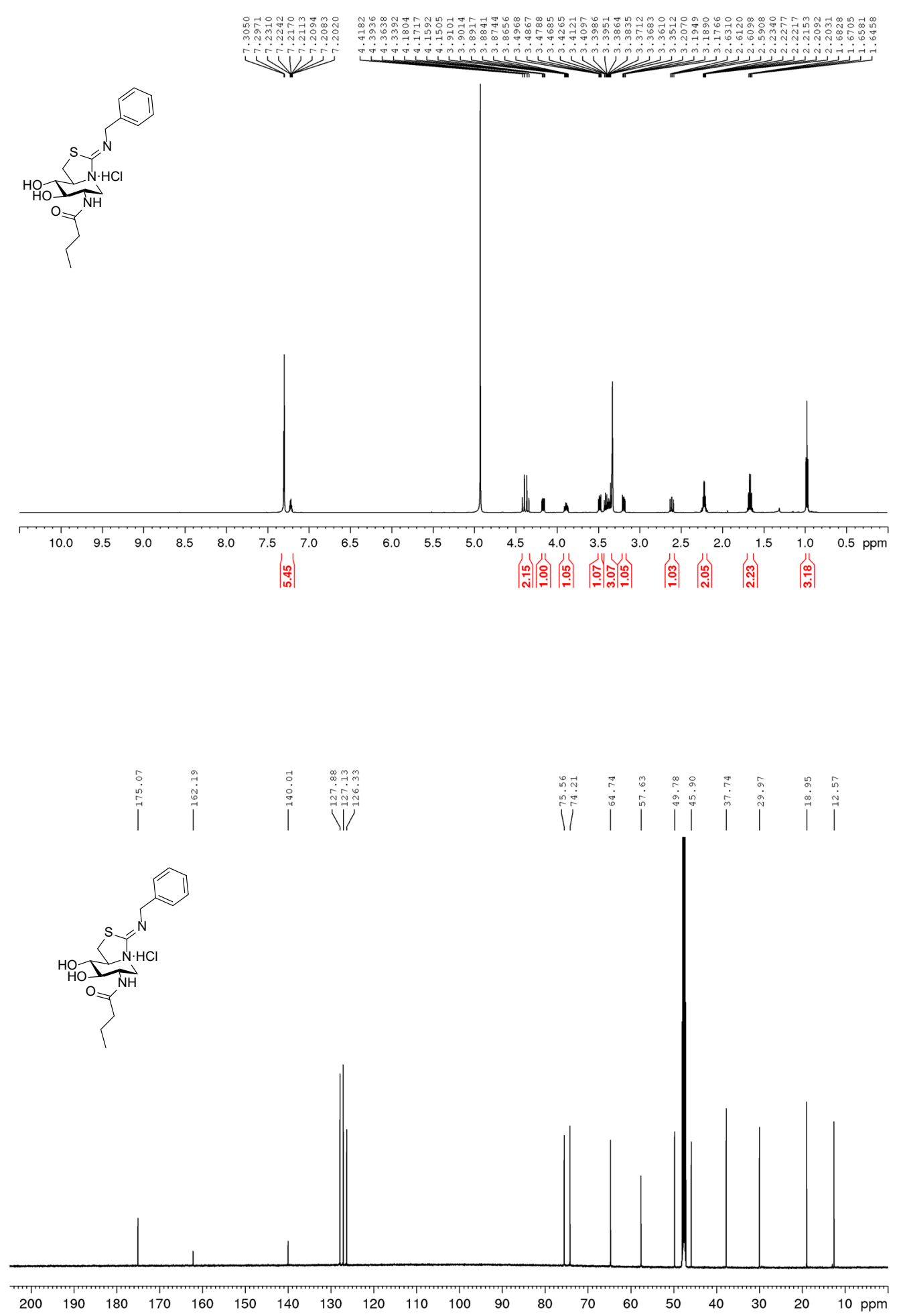

Supplemental Figure 58. ${ }^{1} \mathrm{H}$ NMR and ${ }^{13} \mathrm{C}$ NMR (600 MHz, $\left.150 \mathrm{MHz} \mathrm{CD}_{3} \mathrm{OD}\right)$ of 29 

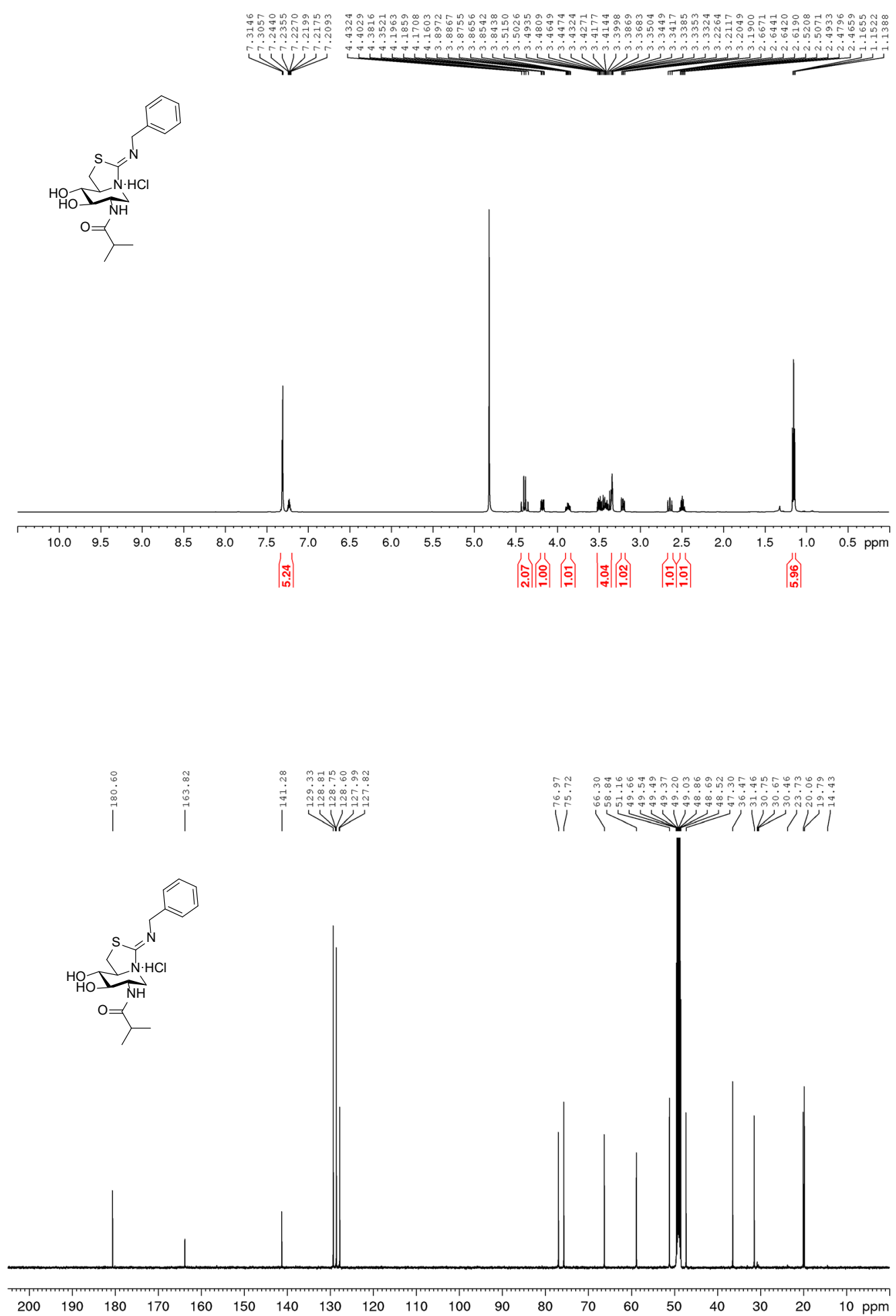

Supplemental Figure 59. ${ }^{1} \mathrm{H}$ NMR and ${ }^{13} \mathrm{C}$ NMR (500 MHz, $\left.125.7 \mathrm{MHz} \mathrm{CD}_{3} \mathrm{OD}\right)$ of 30 

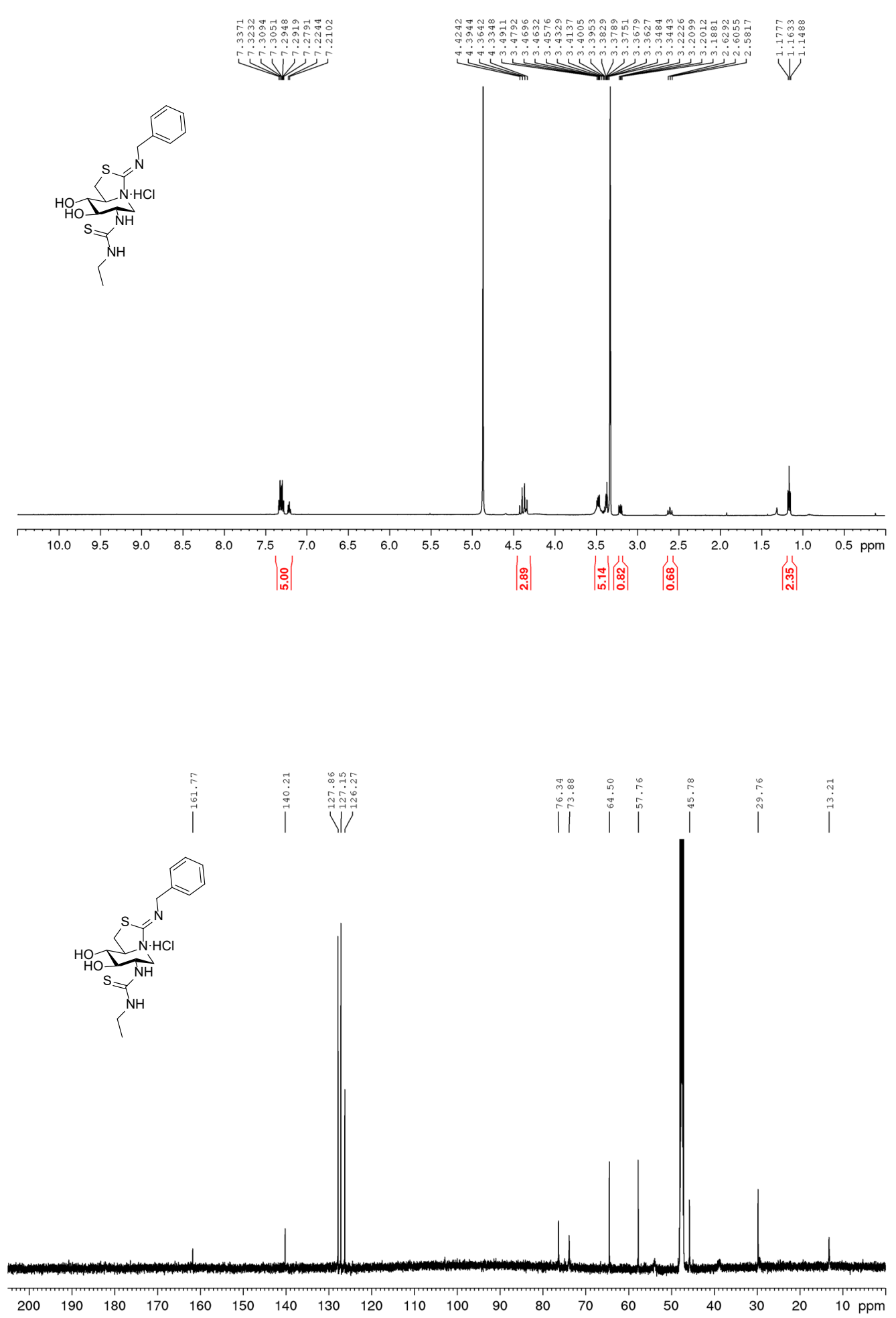

Supplemental Figure 60. ${ }^{1} \mathrm{H}$ NMR and ${ }^{13} \mathrm{C}$ NMR (600 MHz, $\left.150 \mathrm{MHz} \mathrm{CD}_{3} \mathrm{OD}\right)$ of 31 

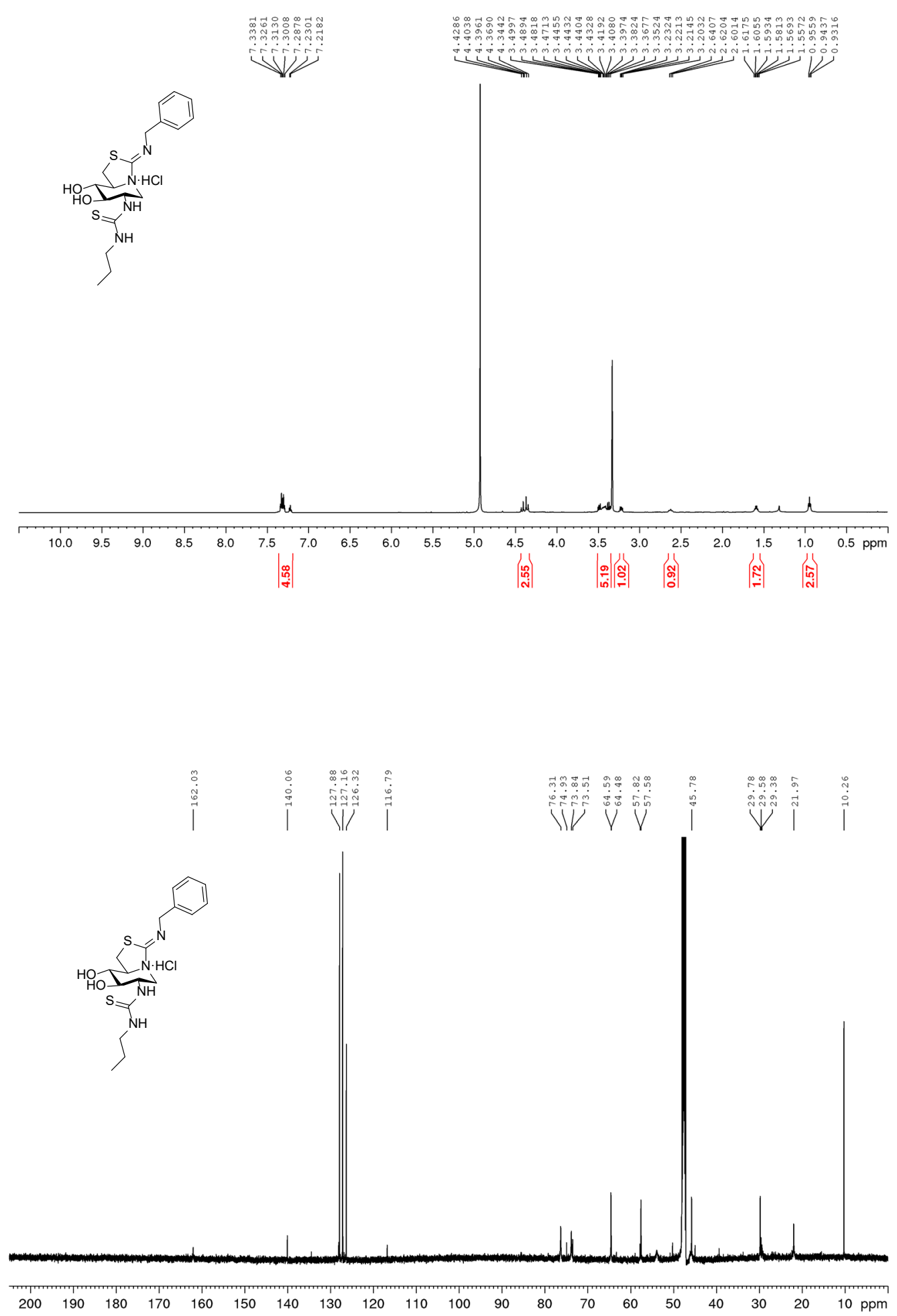

Supplemental Figure 61. ${ }^{1} \mathrm{H}$ NMR and ${ }^{13} \mathrm{C}$ NMR (600 MHz, $\left.150 \mathrm{MHz} \mathrm{CD}_{3} \mathrm{OD}\right)$ of 32 


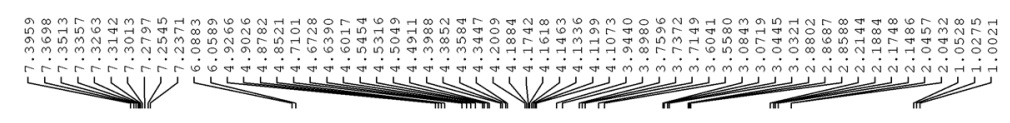

$$
\overbrace{A c 0}^{O A C}
$$
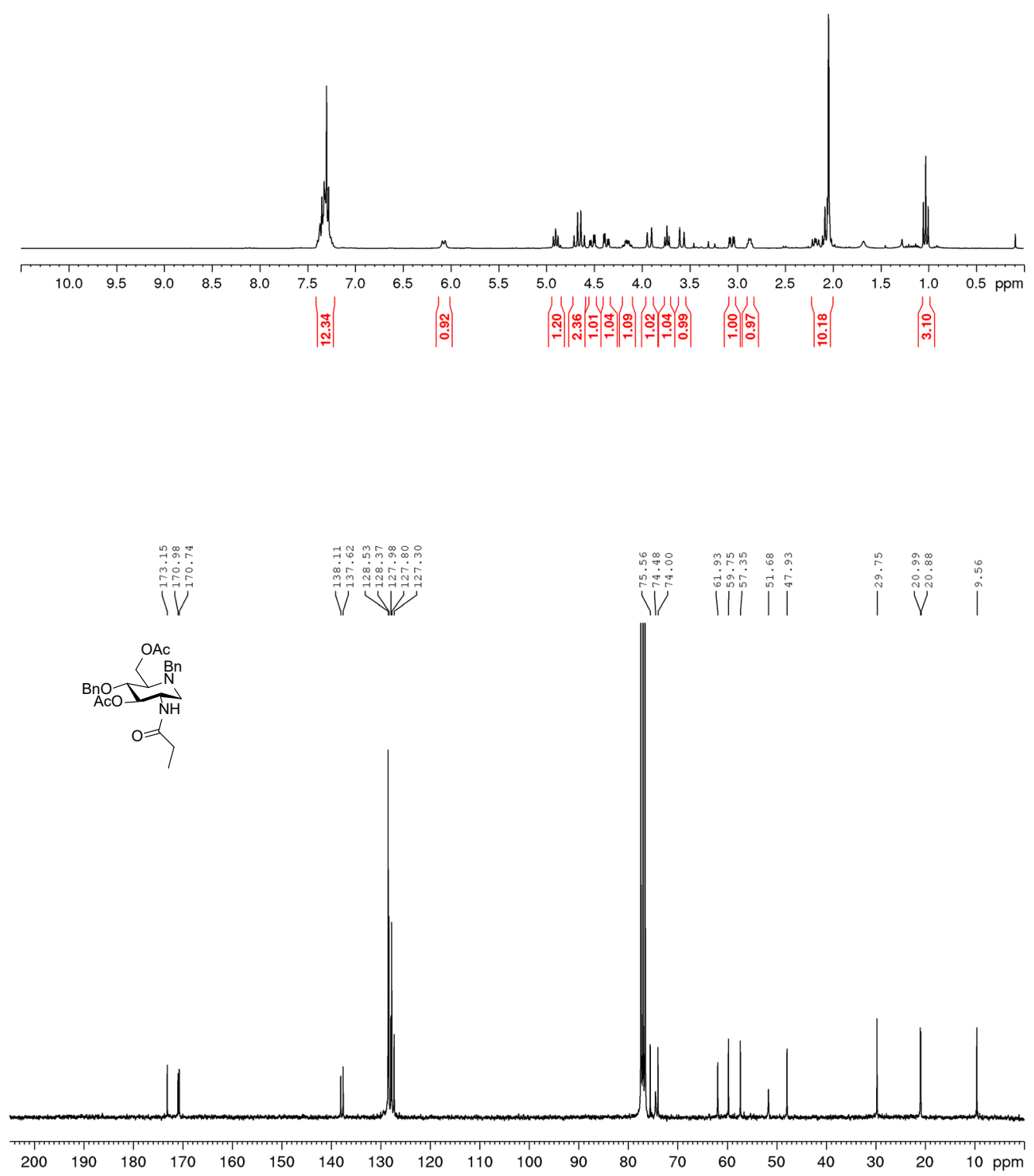

Supplemental Figure 62. ${ }^{1} \mathrm{H}$ NMR and ${ }^{13} \mathrm{C}$ NMR $\left(300 \mathrm{MHz}, 75.5 \mathrm{MHz} \mathrm{CD}_{3} \mathrm{OD}\right)$ of 33 

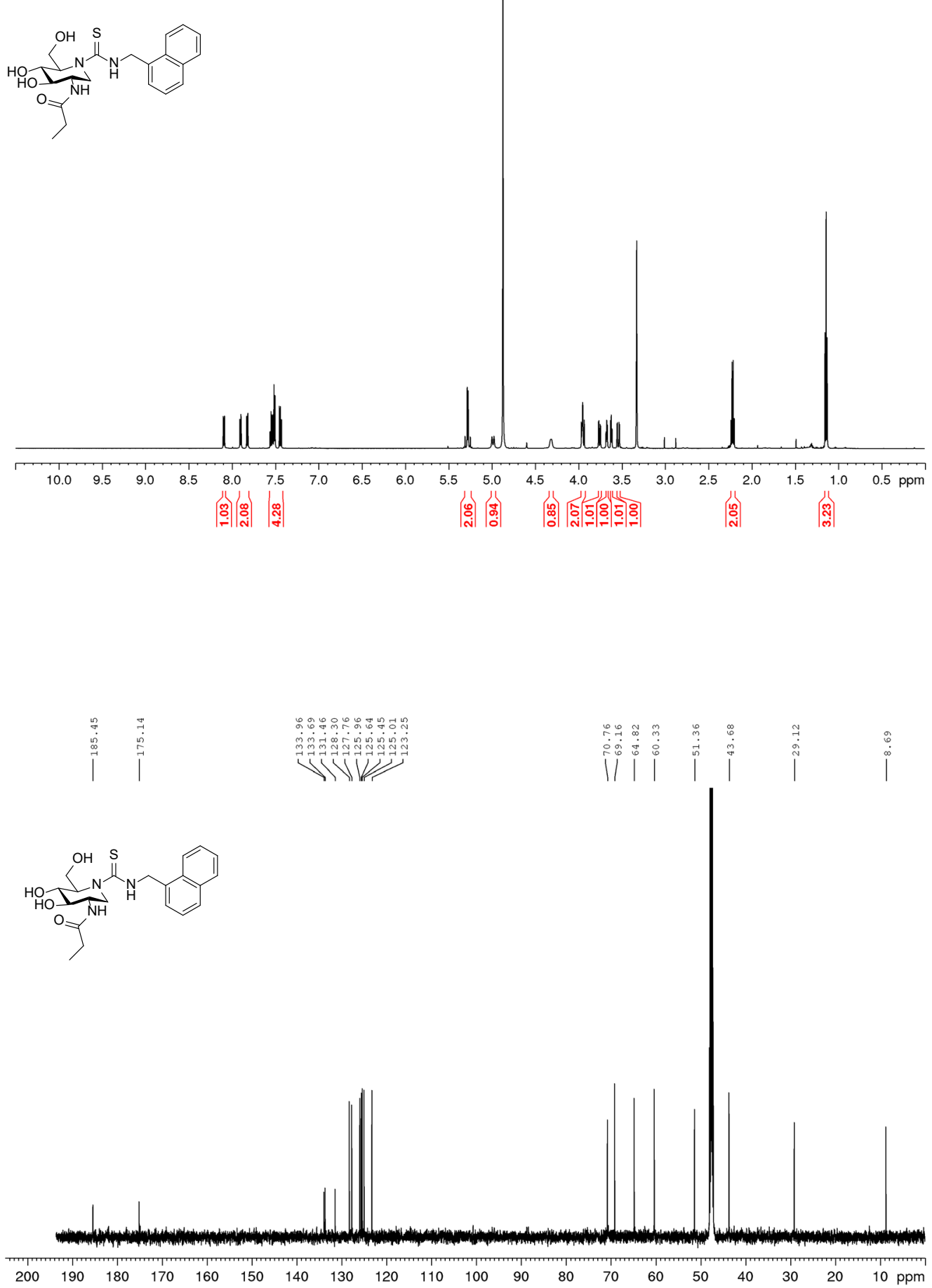

Supplemental Figure 63. ${ }^{1} \mathrm{H} \mathrm{NMR}$ and ${ }^{13} \mathrm{C} \mathrm{NMR}\left(600 \mathrm{MHz}, 150 \mathrm{MHz} \mathrm{CD}_{3} \mathrm{OD}\right)$ of 35 

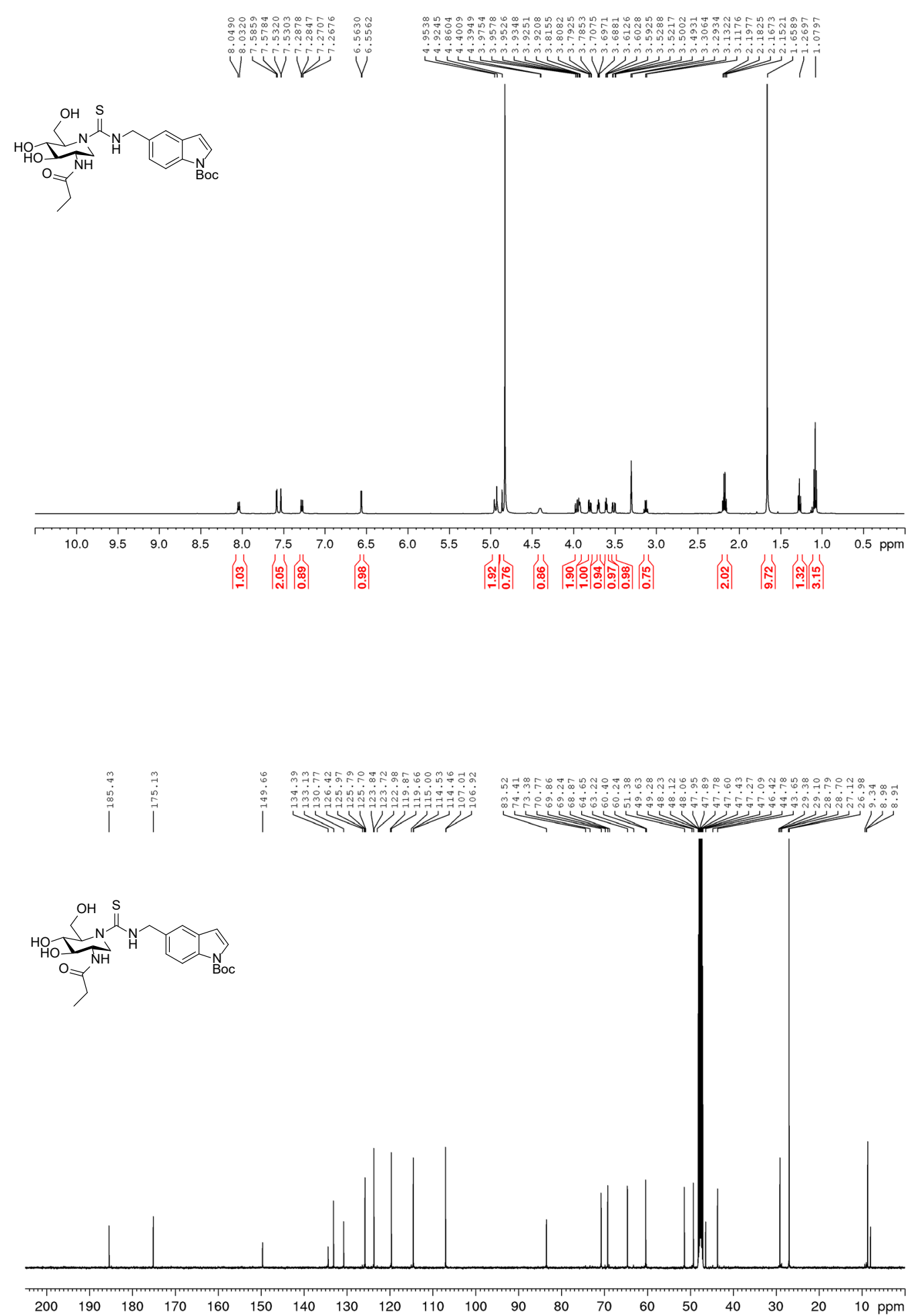

Supplemental Figure 64. ${ }^{1} \mathrm{H}$ NMR and ${ }^{13} \mathrm{C}$ NMR $\left(500 \mathrm{MHz}, 125.7 \mathrm{MHz} \mathrm{CD}_{3} \mathrm{OD}\right)$ of 36 

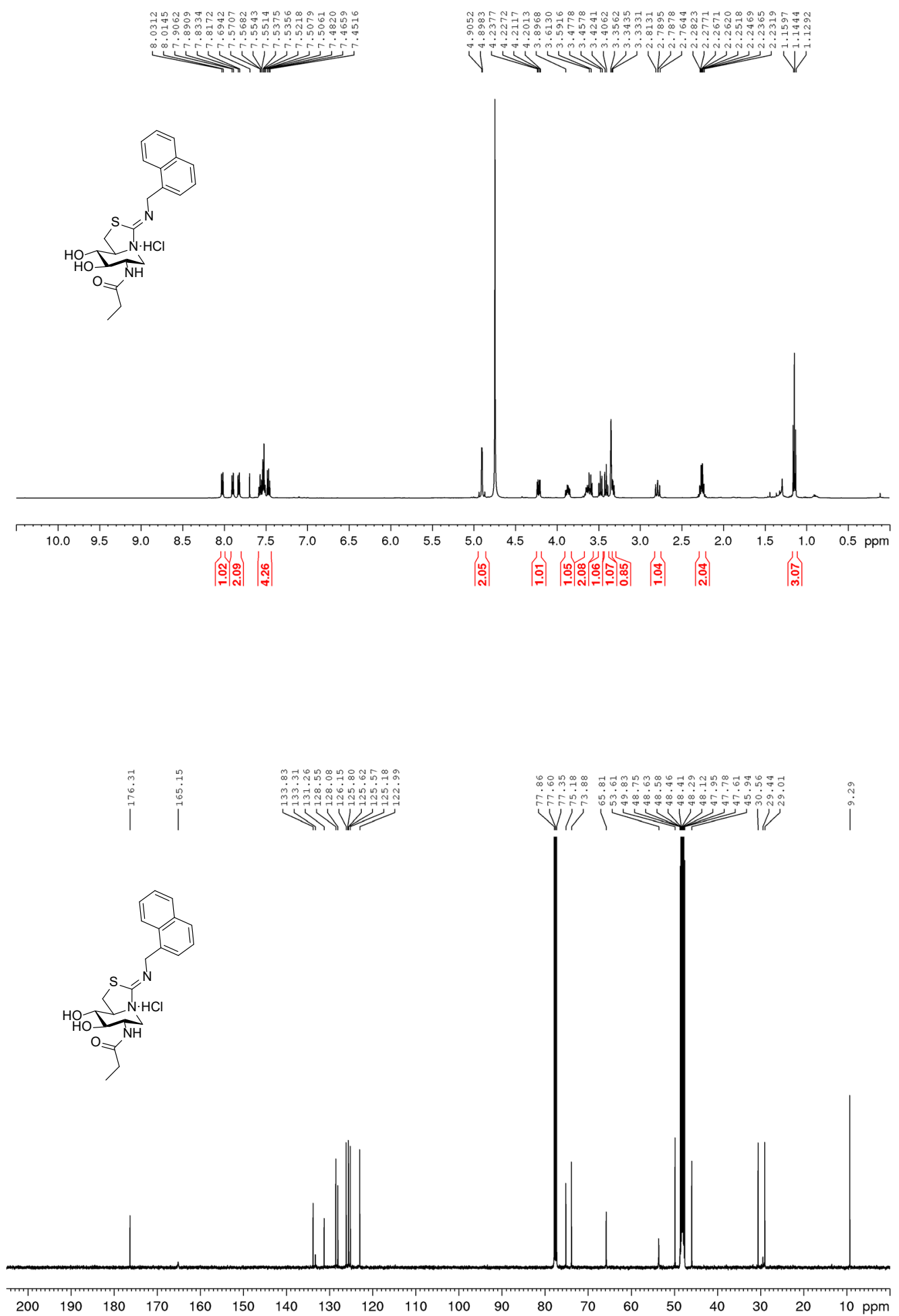

Supplemental Figure 65. ${ }^{1} \mathrm{H}$ NMR and ${ }^{13} \mathrm{C}$ NMR $\left(500 \mathrm{MHz}, 125.7 \mathrm{MHz}\right.$ 1:1 $\mathrm{CD}_{3} \mathrm{OD}-$ $\mathrm{CDCl}_{3}$ ) of $\mathbf{3 7}$ 

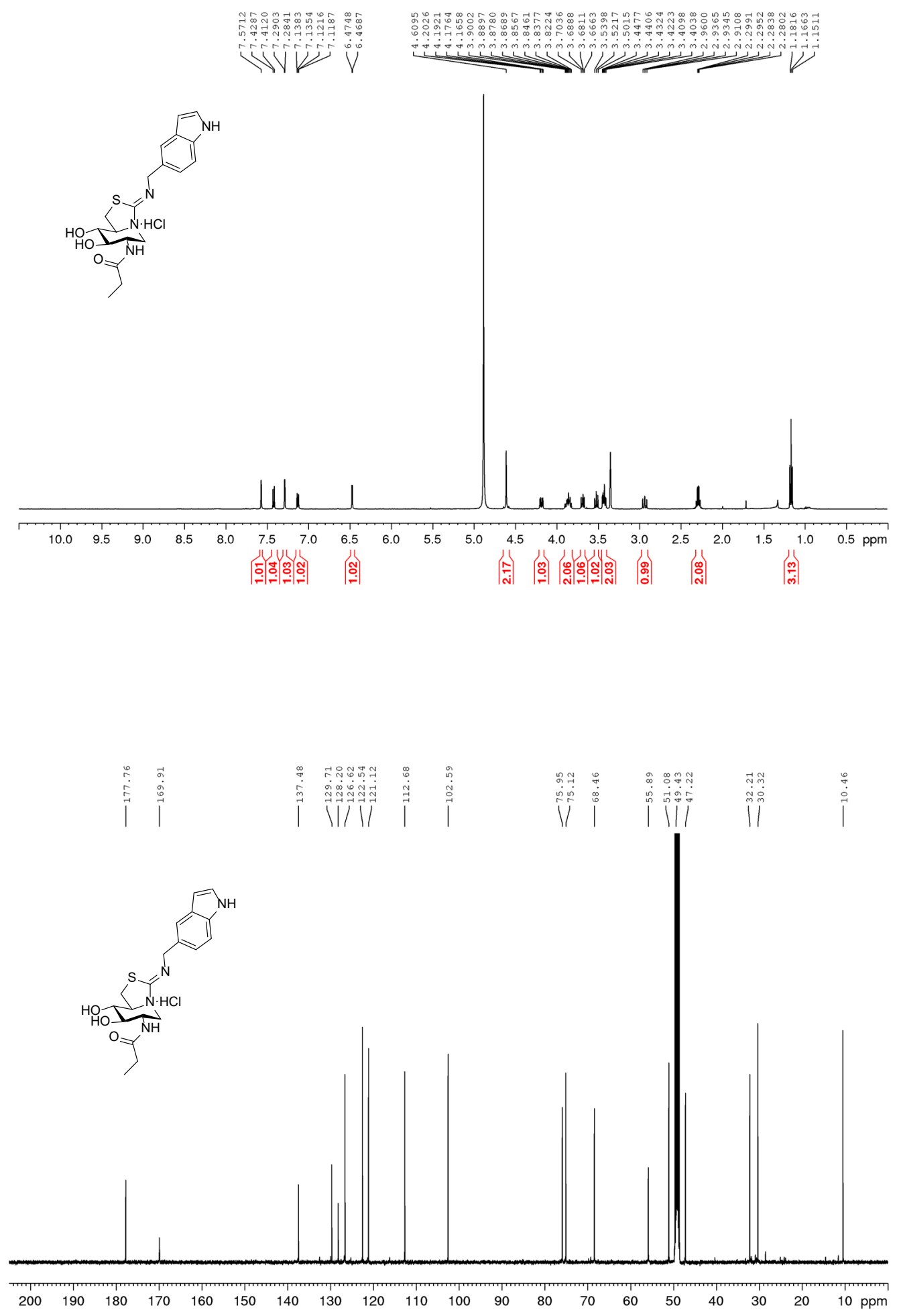

Supplemental Figure 66. ${ }^{1} \mathrm{H}$ NMR and ${ }^{13} \mathrm{C}$ NMR $\left(500 \mathrm{MHz}, 125.7 \mathrm{MHz} \mathrm{CD}_{3} \mathrm{OD}\right)$ of 38 

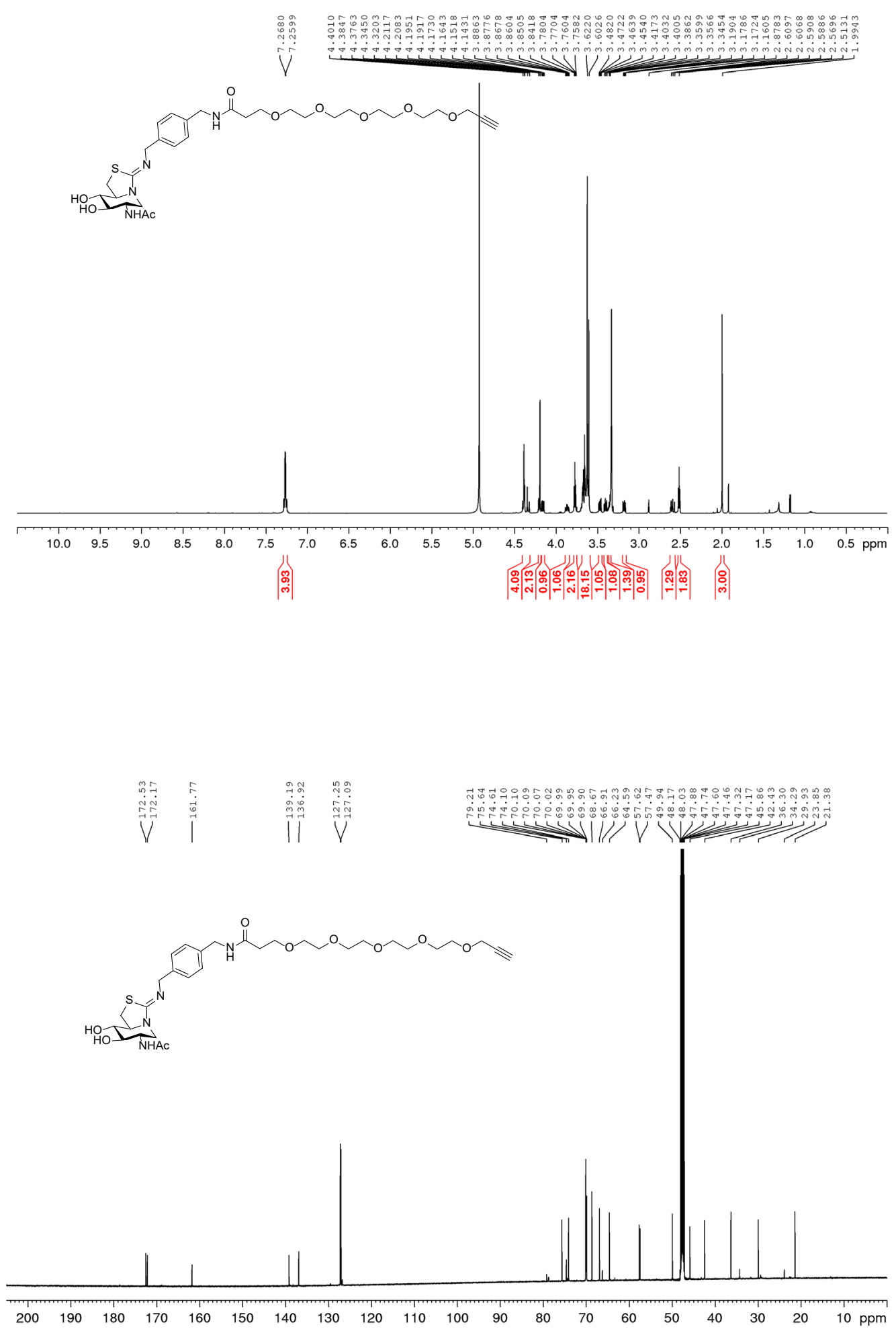

Supplemental Figure 67. ${ }^{1} \mathrm{H}$ NMR and ${ }^{13} \mathrm{C}$ NMR (600 MHz, $\left.150 \mathrm{MHz} \mathrm{CD}_{3} \mathrm{OD}\right)$ of 39 

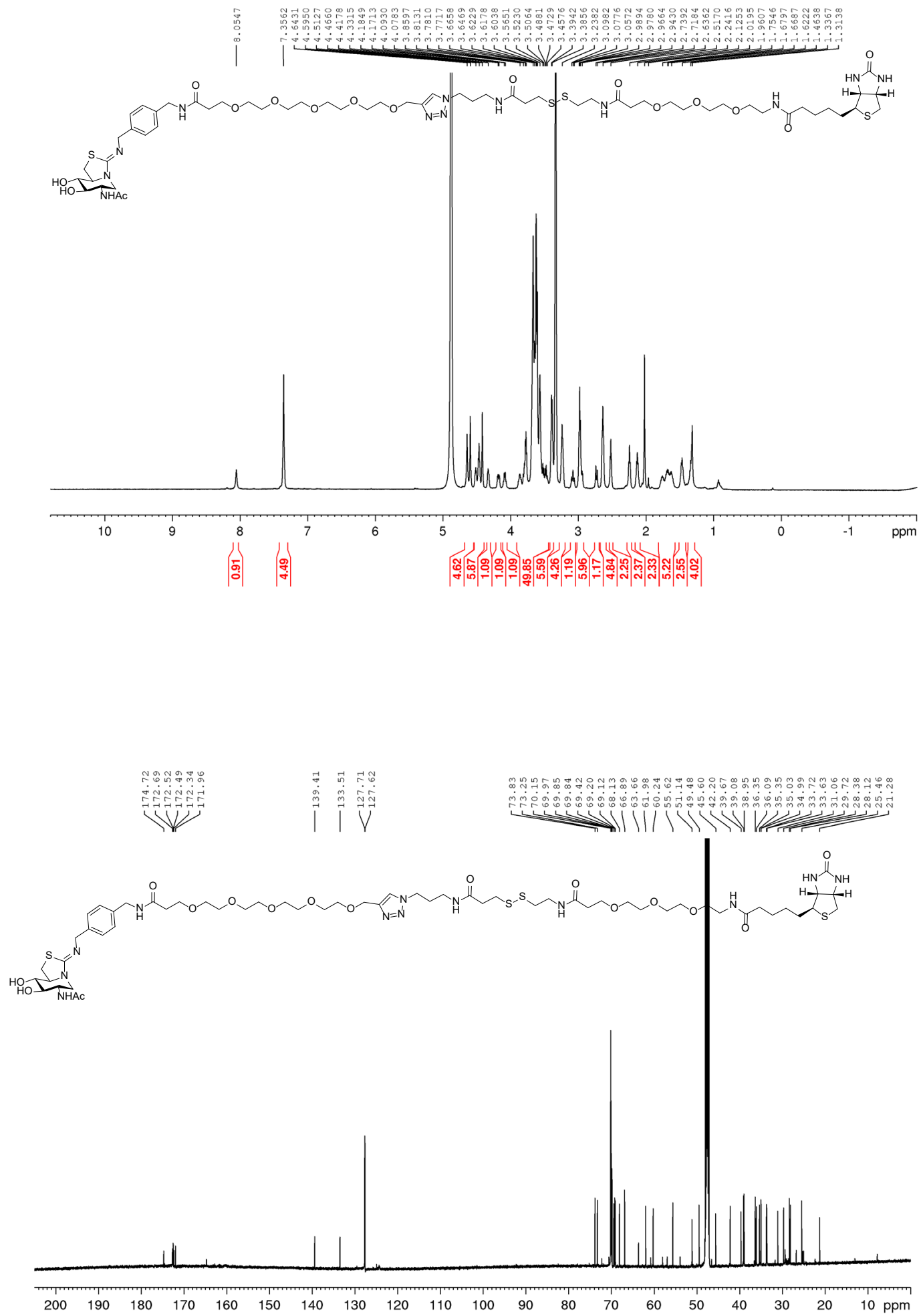

Supplemental Figure 68. ${ }^{1} \mathrm{H}$ NMR and ${ }^{13} \mathrm{C}$ NMR (600 MHz, $\left.150 \mathrm{MHz} \mathrm{CD}_{3} \mathrm{OD}\right)$ of 40 


\section{References}

(1) A. F. G. Glawar, D. Best, B. J. Ayers, S. Miyauchi, S. Nakagawa, M. AguilarMoncayo, J. M. García Fernández, C. Ortiz Mellet, E. V. Crabtree, T. D. Butters, F. X. Wilson, A. Kato, G. W. J. Fleet. Scalable Syntheses of Both Enantiomers of DNJNAc and DGJNAc from Glucuronolactone: The Effect of N-Alkylation on Hexosaminidase Inhibition. Chem. Eur. J. 2012, 18, 9341-9359.

(2) J. R. Thomas, X. Liu, P. J. Hergenrother. Size-Specific Ligands for RNA Hairpin Loops. J. Am. Chem. Soc. 2005, 127, 12434-12435.

(3) K. N. Farrugia, D. Makuc, A. Podborska, K. Szaciłowski, J. Plavec, D. C. Magri. Colorimetric Naphthalene-Based Thiosemicarbazide Anion Chemosensors with an Internal Charge Transfer Mechanism. Eur. J. Org. Chem. 2016, 16, 4415-4422.

(4) K. A. Stubbs, J. P. Bacik, G. E. Perley-Robertson, G. E. Whitworth, T. M. Gloster, D. J. Vocadlo, B. L. Mark. The Development of Selective Inhibitors of Nagz: Increased Susceptibility of Gram-Negative Bacteria to $\beta$-Lactams. ChemBioChem 2013, 14, 19731981.

(5) K. N. Lau, H. F. Chow, M. C. Chan, K. W. Wong. Dendronized Polymer Organogels from Click Chemistry: A Remarkable Gelation Property Owing to Synergistic Functional-Group Binding and Dendritic Size Effects. Angew. Chem. Int. Ed. 2008, 47, 6912-6916.

(6) C. Roth, S. Chan, W. A. Offen, G. R. Hemsworth, L. I. Willems, D. T. King, V. Varghese, R. Britton, D. J. Vocadlo, G. J. Davies. Structural and Functional Insight into Human O-GlcNAcase. Nat. Chem. Biol. 2017, 13, 610-612.

(7) R. J. Dennis, E. J. Taylor, M. S. Macauley, K. A. Stubbs, J. P. Turkenburg, S. J. Hart, G. N. Black, D. J. Vocadlo, G. J. Davies. Structure and Mechanism of a Bacterial $\beta$-Glucosaminidase Having O-GlcNAcase Activity. Nat. Struct. Mol. Biol. 2006, 13, $365-$ 371.

(8) G. Winter, J. Appl. Xia2 : An Expert System for Macromolecular Crystallography Data Reduction. Crystallogr. 2010, 43, 186-190. 
(9) P. Evans. Scaling and Assessment of Data Quality. Acta Crystallogr. Sect. D Biol. Crystallogr. 2006, 62, 72-82.

(10) P. R. Evans, G. N. Murshudov. How Good Are My Data and What Is the Resolution? Acta Crystallogr. Sect. D Biol. Crystallogr. 2013, 69, 1204-1214.

(11) M. D. Winn, C. C. Ballard, K. D. Cowtan, E. J. Dodson, P. Emsley, P. R. Evans, R. M. Keegan, E. B. Krissinel, A. G. W. Leslie, A. McCoy, S. J. McNicholas, G. N. Murshudov, N. S. Pannu, E. A. Potterton, H. R. Powell, R. J. Read, A. Vagin, K. S. Wilson. Overview of the CCP 4 Suite and Current Developments. Acta Crystallogr. Sect. D Biol. Crystallogr. 2011, 67, 235-242.

(12) A. Vagin, A. Teplyakov. Molecular Replacement with MOLREP. Acta Crystallogr. Sect. D Biol. Crystallogr. 2010, 66, 22-25.

(13) P. Emsley, B. Lohkamp, W. G. Scott, K. Cowtan. Features and Development of Coot. Acta Crystallogr. Sect. D Biol. Crystallogr. 2010, 66, 486-501.

(14) G. N. Murshudov, A. A. Vagin, E. J. Dodson. Refinement of Macromolecular Structures by the Maximum-Likelihood Method. Acta Crystallogr. Sect. D Biol. Crystallogr. 1997, 53, 240-255.

(15) G. N. Murshudov, P. Skubák, A. A. Lebedev, N. S. Pannu, R. A. Steiner, R. A. Nicholls, M. D. Winn, F. Long, A. A. Vagin. REFMAC 5 for the Refinement of Macromolecular Crystal Structures. Acta Crystallogr. Sect. D Biol. Crystallogr. 2011, 67, 355-367.

(16) N. S. Pannu, G. N. Murshudov, E. J. Dodson, R. J. Read. Incorporation of Prior Phase Information Strengthens Maximum-Likelihood Structure Refinement. Acta Crystallogr. Sect. D Biol. Crystallogr. 1998, 54, 1285-1292.

(17) A. A. Vagin, R. A. Steiner, A. A. Lebedev, L. Potterton, S. McNicholas, F. Long, G. N. Murshudov. REFMAC 5 Dictionary: Organization of Prior Chemical Knowledge and Guidelines for Its Use. Acta Crystallogr. Sect. D Biol. Crystallogr. 2004, 60, $2184-$ 2195.

(18) C. J. Williams, J. J. Headd, N. W. Moriarty, M. G. Prisant, L. L. Videau, L. N. Deis, V. Verma, D. A. Keedy, B. J. Hintze, V. B. Chen, S. Jain, S. M. Lewis, W. B. 
Arendall, J. Snoeyink, P. D. Adams, S. C. Lovell, J. S. Richardson, D. C. Richardson. MolProbity: More and Better Reference Data for Improved All-Atom Structure Validation. Protein Sci. 2018, 27, 293-315.

(19) J. Agirre, J. Iglesias-Fernández, C. Rovira, G. J. Davies, K. S. Wilson, K. D. Cowtan. Privateer: Software for the Conformational Validation of Carbohydrate Structures. Nat. Struct. Mol. Biol. 2015, 22, 833-834.

(20) S. McNicholas, E. Potterton, K. S. Wilson, M. E. M. Noble. Presenting Your Structures: The CCP 4 Mg Molecular-Graphics Software. Acta Crystallogr. Sect. D Biol. Crystallogr. 2011, 67, 386-394. 